\title{
APPLICATION OF PULSE SPARK DISCHARGES FOR SCALE PREVENTION AND CONTINUOUS FILTRATION METHODS IN COAL-FIRED POWER PLANT
}

\author{
Final Technical Report
}

October 2008 - June 2012

\author{
Prepared for \\ U.S. Department of Energy \\ National Energy Technology Laboratory \\ 3610 Collins Ferry Road \\ P.O. Box 880 \\ Morgantown, WV 26507-0880
}

July 24,2012

\author{
Submitted by \\ Young I Cho and Alexander A. Fridman \\ Department of Mechanical Engineering and Mechanics \\ Drexel University \\ 3141 Chestnut St., Philadelphia, PA 19104
}

DOE Award No: DEFC2608NT0005308

This work was sponsored by The Innovations for Existing Plants (IEP) Program National Energy Technology Laboratory (NETL), Office of Fossil Energy, U.S. Department of Energy 


\section{Disclaimer}

This report was prepared as an account of work sponsored by an agency of the United States Government. Neither the United States nor any agency thereof, nor any of their employees, makes any warranty, express or implied, or assumes any legal liability or responsibility for the accuracy, completeness, or usefulness of any information, apparatus, product, or process disclosed, or represents that its use would not infringe privately owned rights. Reference herein to any specific commercial product, process, or service by trade name, trademark, manufacturer, or otherwise does not necessarily constitute or imply its endorsement, recommendation, or favoring by the United States Government or any agency thereof. The views and opinions of authors expressed herein do not necessarily state or reflect those of the United States Government or any agency thereof. 


\section{Abstract}

The overall objective of the present work was to develop a new scale-prevention technology by continuously precipitating and removing dissolved mineral ions (such as calcium and magnesium) in cooling water while the COC could be doubled from the present standard value of 3.5. The hypothesis of the present study was that if we could successfully precipitate and remove the excess calcium ions in cooling water, we could prevent condenser-tube fouling and at the same time double the COC. The approach in the study was to utilize pulse spark discharges directly in water to precipitate dissolved mineral ions in recirculating cooling water into relatively large suspended particles, which could be removed by a self-cleaning filter.

The present study began with a basic scientific research to better understand the mechanism of pulse spark discharges in water and conducted a series of validation experiments using hard water in a laboratory cooling tower. Task 1 of the present work was to demonstrate if the spark discharge could precipitate the mineral ions in water. Task 2 was to demonstrate if the selfcleaning filter could continuously remove these precipitated calcium particles such that the blowdown could be eliminated or significantly reduced. Task 3 was to demonstrate if the scale could be prevented or minimized at condenser tubes with a COC of 8 or (almost) zero blowdown. In Task 1, we successfully completed the validation study that confirmed the precipitation of dissolved calcium ions in cooling water with the supporting data of calcium hardness over time as measured by a calcium ion probe. In Task 2 , we confirmed through experimental tests that the self-cleaning filter could continuously remove precipitated calcium particles in a simulated laboratory cooling tower such that the blowdown could be eliminated or significantly reduced. In addition, chemical water analysis data were obtained which were used to confirm the COC calculation. In Task 3, we conducted a series of heat transfer fouling tests using a condenser heat exchanger in the laboratory cooling tower, from which we confirmed that the plasma water treatment technology could prevent or significantly mitigate mineral foulings in condenser tubes when compared with the no-treatment case.

With the completion of the present work, a cooling water treatment technology using pulse spark discharges is currently ready for field-validation tests. The plasma water treatment technology is a true mechanical water softener with almost no maintenance, which continuously converts hard water to soft water spending a relatively small amount of energy. Such a mechanical water softener could find wide-spread applications to solve hard water problems both in industry and at home.

Keywords: water treatment; cooling water; blow down; spark discharge; shockwave; self-cleaning filter; plasma water treatment; mineral fouling; calcium scaling 


\section{Acknowledgements}

Drexel University wishes to acknowledge US Department of Energy, National Energy Technology Laboratory for its financial support.

\section{Point of Contact}

Further information on the project and the technology could be obtained from:

Barbara Carney, Project Officer/Manager,

Department of Energy, National Energy Technology Laboratory

Morgantown, WV, 304-285-4671

E-mail: barbara.carney@netl.doe.gov 


\section{TABLE OF CONTENTS}

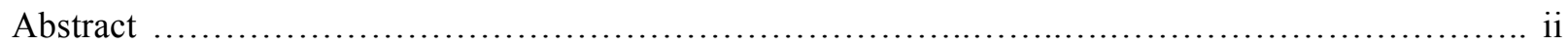

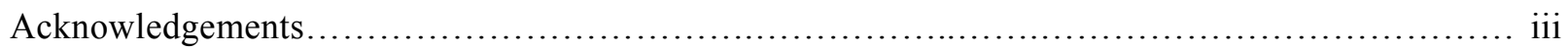

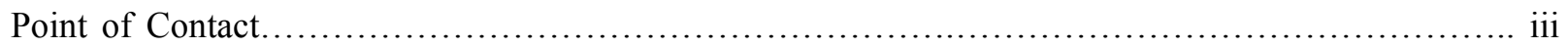

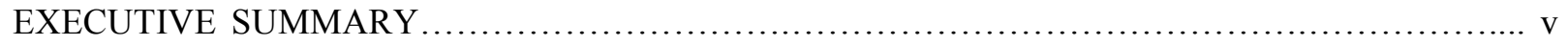

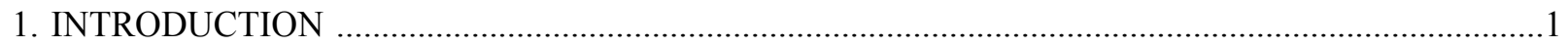

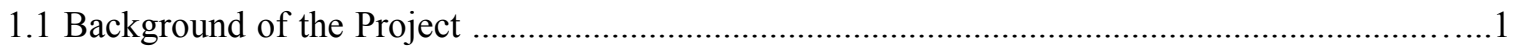

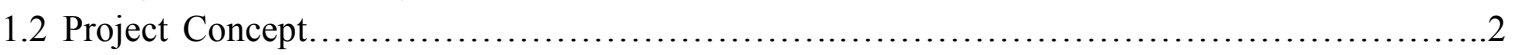

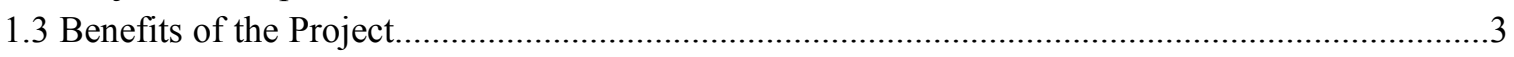

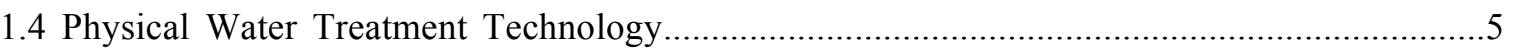

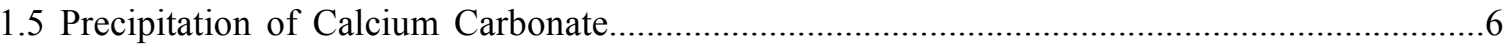

1.6 Plasma Discharges in Water............................................................................... 7

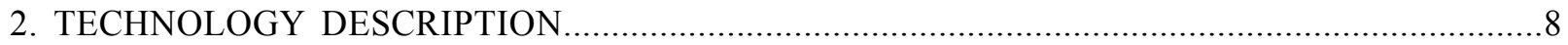

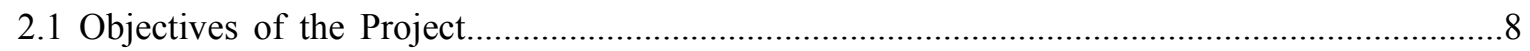

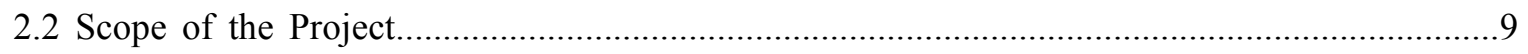

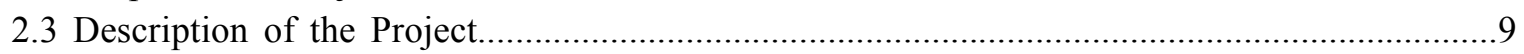

TECHNICAL PERFORMANCE

3. Overview on Plasma Discharge and Cooling Water Treatment..................................................8

3.1 Plasma Generation in Nature and in Laboratory...................................................... 10

3.2 Needs for Plasma Water Treatment.................................................. 11

3.3 Conventional Water Treatment Technologies.................................................................. 12

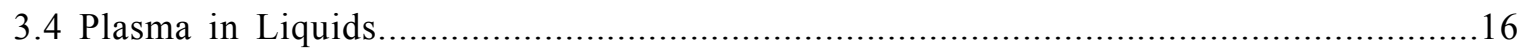

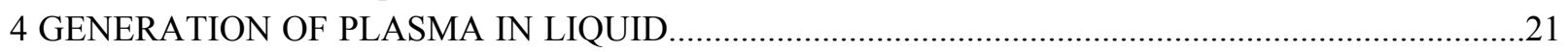

4.1 Partial and Full Discharge in Liquid..........................................................21

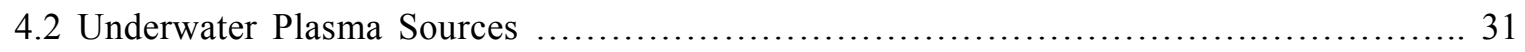

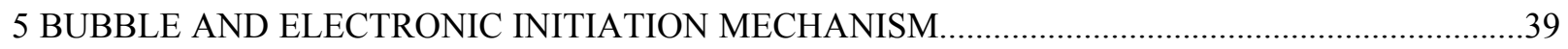

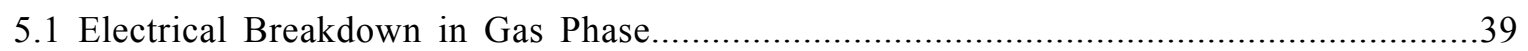

5.2 Electron Avalanche for Electrical Breakdown in Liquid Phase.............................46

5.3 "Bubble Theory" for Electric Breakdown in liquid..............................................50

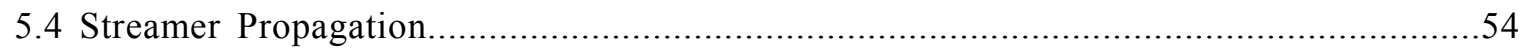

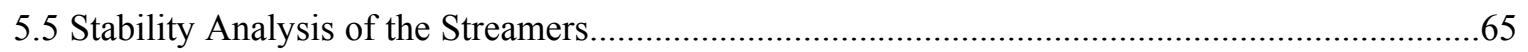

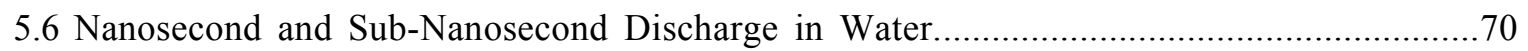

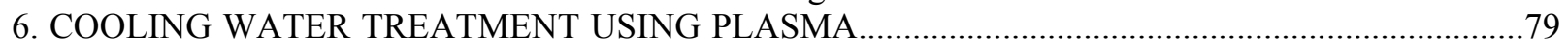

6.1 Self-Cleaning Filtration Technology with Spark Discharge ................................8 82

6.2 Calcium Carbonate Precipitation with Spark Discharge .................................88

6.3. Mechanism of Plasma-Induced Calcium Precipitation ........................................................102

6.4 Economic Analysis on Plasma Water Treatment ........................................... 114

6.5 Application for Mineral Fouling Mitigation in Heat Exchangers............................115

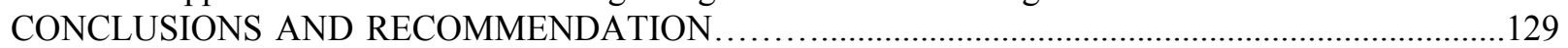

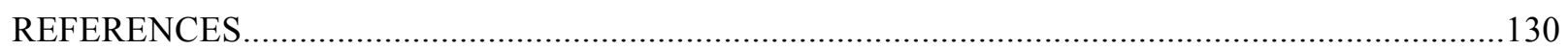

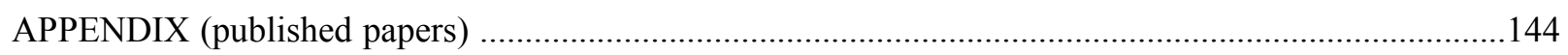




\section{EXECUTIVE SUMMARY}

\section{Background}

Thermoelectric power generation is water intense. The goal of water management program in the Innovations for Existing Plants (IEP) program, managed by the Office of Fossil Energy's National Energy Technology Laboratory (NETL) (Feeley, 2008), was to develop advanced technologies and concepts to ensure that sufficient water could be available to operate coal-based power systems and to minimize potential impacts of plant operations on water quality. The shortterm goal was to have technologies ready for commercial demonstration by 2015 that, when used alone or in combination, could reduce freshwater withdrawal and consumption by $50 \%$ or greater for thermoelectric power plants equipped with wet recirculating cooling technology at a leveled cost of less than $\$ 4.40$ per thousand gallons freshwater conserved. The long-term goal was to have technologies ready for commercial demonstration by 2020 that, when used in combination, could reduce freshwater withdrawal and consumption by $70 \%$ or greater at a leveled cost of less than $\$ 2.90$ per thousand gallons freshwater conserved.

\section{Objectives}

The overall objective of the present work was to develop a new scale-prevention technology by continuously precipitating and removing dissolved mineral ions (such as calcium and magnesium) in cooling water while the COC could be doubled from the present standard value of 3.5. The approach in the study was to utilize the pulse spark discharges to precipitate dissolved mineral ions in recirculating cooling water into relatively large suspended particles, which could be removed by a self-cleaning filter so that the mineral fouling could be prevented in condenser tubes in spite of increased COC operation.

\section{Scope and Benefits}

The scope of the present study was to utilize plasma spark discharges in water to precipitate dissolved mineral ions in recirculating cooling water in a simulated laboratory cooling tower and continuously remove precipitated mineral salts using a self-cleaning filter such that the COC in cooling water could be doubled while preventing mineral fouling on condenser tubes. The hypothesis of the present study was that if we could successfully precipitate and remove the excess calcium ions in cooling water, we could prevent condenser-tube fouling and the same time double the COC. The present study began with a basic scientific research to better understand the mechanism of pulse spark discharges in water and conducted a series of validation experiments using hard water in a laboratory cooling tower.

The most significant benefit of the present work was that the present spark discharge technology allows a cooling tower to operate at a much higher COC than the current standard of 3.5 (Herro and Port, 1993), while preventing or minimizing scales on the condenser tubes. The present plasma treatment technology demonstrated that we could reduce the blowdown by approximately $25 \%$, which was approximately 2.5 million gallons a day in a $1000-\mathrm{MW}$ fossilfueled power plant, accomplishing one of the major DOE's goals of reducing/minimizing 
freshwater withdrawal in thermoelectric power plants. At the completion of the present work, a cooling water treatment technology using pulse spark discharges is now ready for the fieldvalidation tests. The plasma water treatment technology is a true mechanical water softener with almost no maintenance, which continuously converts hard water to soft water spending a relatively small amount of energy. We hope that such a mechanical water softener can find widespread applications to solve hard water problems both in industry and at home.

\section{Tasks Performed}

In Task 1, we conducted a series of tests to demonstrate that the plasma spark discharge could precipitate the mineral ions in water.

In Task 2, we conducted validation experiments in a simulated cooling tower to demonstrate that the self-cleaning filter using spark discharge could continuously remove these precipitated calcium particles such that the blowdown could be eliminated or significantly reduced.

In Task 3, we conducted heat transfer fouling experiments to demonstrate that mineral scale could be prevented or minimized at condenser tubes with a COC of 8 or (almost) zero blowdown.

\section{Results}

Task 1 - Precipitation of dissolved calcium ions using spark discharge (Year 1)

One of the primary reasons why thermoelectric generation accounted for approximately $39 \%$ (136 billion gallons per day [BGD]) of all freshwater withdrawals in the nation in 2000 (Feeley, 2008 ) is that the concentration of the mineral ions such as calcium and magnesium in the circulating cooling water increases with time as pure water evaporates to remove heat from condenser tubes. In order to maintain a COC of 3.5 in the cooling water to avoid mineral fouling problem, one needs to continuously blowdown cooling water and replace it with makeup water. In Task 1 we demonstrated that we could precipitate and remove the dissolved calcium ions with spark discharges such that blowdown was not needed to maintain a constant calcium ion level in cooling water.

The precipitation of excess calcium ions in cooling water depended on a number of parameters. In the water side, the $\mathrm{pH}$, the level of supersaturation, and its temperature could affect the precipitation rate of dissolved calcium ions in cooling water. In the power supply side, the frequency $(\mathrm{Hz})$, power level (voltage), pulse duration (nanosecond), and the rising time of pulse could affect the rate of precipitation. In Task 1, we conducted a study to examine the effects of these variables on the precipitation process of excess calcium ions in cooling water by modeling and experimental study. 
Task 2: Validation experiments to increase COC (Year 2)

The second specific goal was to demonstrate if the present technology could increase the COC through a continuous precipitation of calcium ions and removing them with the selfcleaning filter.

In Task 2, we built a laboratory cooling tower, where pure water continuously evaporated as we added heat through a condenser heat exchanger. In the laboratory tower, the amount of water lost by evaporation, wind, and blowdown was automatically replaced by a makeup water valve, which was a floating valve located at the tower sump. The cooling tower had an automatic blowdown capability with a solenoid valve which was turned on-off by a preset conductivity meter. We simulated a typical cooling tower operation using the tap water supplied by the City of Philadelphia. We successfully demonstrated that we could increase COC above 3.5 in cooling water by continuous precipitation of calcium ions and removal of them with the self-cleaning filter.

Task 3: Validation experiments for scale prevention (Year 3)

The third specific goal in the present study was to investigate if mineral scale could be prevented or minimized at condenser tubes with a COC of 8 or almost zero blowdown. We conducted a series of heat transfer fouling tests using a condenser heat exchanger in the laboratory cooling tower. The fouling resistance was experimentally determined by measuring the inlet and outlet temperatures at both cooling-water side and hot-fluid side. The fouling resistance obtained with the present scale-prevention technology was compared with the no-treatment case as well as the scale-free case. We successfully demonstrated that the mineral fouling in condenser tubes could significantly mitigated even in the cases of COC of 8 as well as no blowdown.

In addition, we have conducted tests to check if spark discharges could kill microorganisms in water. In inactivation tests with $E$. coli in water, an approximately 3.5-log reduction in bacterial concentration was obtained after applying a total of 100 pulses to water having a high $E$. coli concentration of $10^{8.5} \mathrm{CFU} / \mathrm{ml}$ of water. The ability of spark discharge technology to sterilize bio-contaminated wastewater opens a new opportunity to treat various impaired water for cooling water. 


\section{Chapter 1 INTRODUCTION}

\subsection{Background of the Project}

The U. S. Department of Energy (DOE) has established a set of national priorities that includes the goal to promote secure, competitive, and environmentally responsible energy systems that serve the needs of the public. The Innovations for Existing Plants (IEP) program (Feeley, 2008), managed by the Office of Fossil Energy's National Energy Technology Laboratory (NETL), provides technological solutions to the myriad of environmental issues (air, solid, and water) affecting the existing fleet of coal-based power plants representing more than 320 gigawatts $(\mathrm{GW})$ of generating capacity.

Significant quantities of water were necessary for the generation of electrical energy by coal-fired power plants. In fact, each $\mathrm{kWh}$ of thermoelectric generation requires approximately 25 gallons of water (Feeley, 2008). According to USGS's Estimated Use of Water in the United States in 2000, thermoelectric power generation (39\%) ranks only slightly behind irrigation (40\%) as the largest source of freshwater withdrawals in the United States, withdrawing over 136 billion gallons per day primarily for cooling purposes. When discussing water and thermoelectric generation, it was important to distinguish between water use and water consumption. Water use represents the total water withdrawal from a source and water consumption represents the amount of that withdrawal that was not returned to the source. Although thermoelectric generation was the second largest user of water on a withdrawal basis, it was only responsible for approximately $3 \%$ of the total 100 billion gallons per day of freshwater consumed in 1995.

Growing concerns about water availability along with current and future water-related environmental regulations and requirements could impact both the permitting and operation of coal-based power systems. In response to these national energy sustainability and security challenges, the IEP Program has been proactive through a water strategy directed at developing technologies and approaches to better manage how power plants use and impact freshwater resources so that one could reduce the amount of freshwater needed by power plants and minimize potential water quality impacts.

The overall goal of the U.S. DOE/OFE's Innovations for Existing Plants (IEP) Program was to enhance the efficiency and environmental performance of the existing fleet of coal-fired power plants relative to the emission of $\mathrm{CO}_{2}$ and the management of water. More specifically, the objective of the program under the Department of Energy (DOE), National Energy Technology Laboratory (NETL) entitled "Research And Development Of Advanced Technologies And Concepts For Minimization Of Freshwater Withdrawal And Consumption In Coal-Based Thermoelectric Power Plants" was to secure applications for advanced technologies and concepts to reduce/minimize freshwater withdrawal and consumption by coal-based power systems so that low-cost electricity becomes continuously available from coal in light of growing demands on limited freshwater resources.

One of the critical parameters that adversely affect the efficiency of the power plants was the condenser tube fouling by mineral ions such as calcium and magnesium. Since calcium 
carbonate $\mathrm{CaCO}_{3}$ problem was most common in cooling water, we use the word "calcium scale" to refer all scales caused by mineral ions in the report. In order to prevent or minimize the condenser tube fouling, the concentration of cycle (COC) in wet recirculation cooling systems was often kept at 3.5. Increasing the cycles of concentration could reduce the amount of makeup water required due to the reduction in blowdown frequency. The COC was directly tied to the condenser tube fouling. If one could prevent or minimize the condenser tube fouling, then we could increase the COC.

\subsection{Project Concept}

The present study attempted to introduce an innovative water treatment technology utilizing spark discharges in water for scale prevention. The key issue was how to precipitate and remove dissolved calcium ions in recirculating cooling water so that the calcium carbonate scales could be avoided and at the same time the COC could be increased.

More specifically, the present study attempted to utilize a very high voltage $(\sim 40,000 \mathrm{~V}$; with the distance between two electrodes equal to $1 \mathrm{~cm}$ ) to produce spark discharges in water for an extremely brief period (i.e., 10-50 nanoseconds) so that an average electric field of approximately $40,000 \mathrm{~V} / \mathrm{cm}$ could be produced without consuming a large amount of electric energy. Note that the local electric field at the tip of the electrode becomes approximately $1,000,000 \mathrm{~V} / \mathrm{cm}$ as the electric field was inversely proportional to the curvature of the tip. The application of electric discharge in water has been studied for decades, because of its importance both in theory and its practical applications including underwater sound source, sterilization and electric discharge machining, etc (Akiyama et al, 2007). More recently, plasma generators in liquid-phase have been investigated for the purpose of both drinking water and wastewater treatment (Locke et al, 2006).

One of the greatest advantages of these applications against traditional water treatment methods is its low power consumption. Pulsed power is effectively the compression of electrical energy, with pulses being dissipated from the electrode to liquid medium on a timescale of sub-microsecond. This compression means that high peak power output pulses in a range of $10 \mathrm{MW}$ could be generated with a low power consumption of less than $10 \mathrm{~kW}$. Generally, strong electric fields applied to water initiate both chemical and physical changes in water.

On the chemical aspect, electrical discharges could produce a variety of chemically active species, such as $\mathrm{O}^{*}, \mathrm{OH}^{*}, \mathrm{H}^{*}, \mathrm{OH}^{-}, \mathrm{O}_{3}, \mathrm{H}_{2} \mathrm{O}_{2}$, etc. (Arif Malik et al, 2001), all of which are powerful oxidizing agents. Therefore, the in situ electrical discharges in water could provide a means to utilize most of these chemically active species for water treatment and sterilization. On the mechanical aspect, shock waves, UV light and a localized intense thermal energy could be formed, depending upon the properties of water and the magnitude of discharge energy. These physical conditions provide a novel approach for various industrial applications including ferrous and non-ferrous metal recovery from slag waste, extracorporeal shock wave lithotripsy and underwater welding (Mohd Abbas et al, 2007). 
The present work was the first attempt to apply the high-voltage nanosecond plasma discharge directly to cooling water for the purpose of both scale prevention and a self-cleaning filtration. The present work attempted to mechanically precipitate dissolved calcium ions in hard water, a task which can have a monumental impact to our modern society as the hard water problem is the critical technical bottleneck in many processes such as water-cooled condensers, desalination, distillation, etc. In addition, the same spark discharge technology could be used as a sterilization tool, thus preventing biofouling at cooling tower and condenser tubes (due to algae and other microorganisms) which could be a serious problem in summer.

\subsection{Benefits of the Project}

A modern 1,000-MW fossil-fueled power plant with $40 \%$ efficiency would reject 1,500 MW of heat at full load. This is roughly equivalent to $512 \times 10^{6} \mathrm{Btu} / \mathrm{hr}$ and uses about 760,000 $\mathrm{gal} / \mathrm{min}$ of circulating water based on $10^{\circ} \mathrm{C}\left(18^{\circ} \mathrm{F}\right)$ temperature difference in the condenser (ElWakil, 1984). As heat is removed via evaporation of pure water at a cooling tower, the need for the makeup water is about 7,500 gal/min for the typical fossil plant, which results in 10 million gallons a day (El-Wakil, 1984). Thus, if one can operate cooling tower at a COC of 8, one could reduce the blowdown by approximately $25 \%$. This means that the makeup water could be reduced by 2.5 million gallons a day in a 1,000-MW fossil-fueled power plant.

Blowdown is necessary to maintain an appropriate degree of concentration of mineral salts or other impurities in the circulating water (Mohd Abbas et al, 2007). The blowdown in a cooling tower is often controlled based on the measurement of the electric conductivity of the circulating water. Since the amount of the evaporated water is proportional to the total heat of evaporation, one could estimate the water loss due to the evaporation. Note that the evaporation of $1 \mathrm{lb}$ of pure water removes an amount of heat of $970 \mathrm{Btu}$. In a typical power-plant cooling tower, blowdown is normally $25-30 \%$ and wind drift loss is $2-2.5 \%$ of the evaporation losses (Akiyama et al, 2007, Locke et al, 2006). The main reason why one only uses makeup freshwater three or four times is that the calcium hardness must not increase beyond a certain level to avoid scaling problems in condensers.

The most significant benefit of the present work was that the spark discharge technology allows a cooling tower to operate at a much higher COC than the current standard of 3.5 (Herro and Port, 1993), while preventing or minimizing scales on the condenser tubes. Of note was that even though the makeup water to the cooling tower is soft water, the evaporation of pure water increases the calcium concentration in the circulating water to a level that requires blowdown within a week. The high concentration of dissolved calcium ions in the recirculating water causes the condenser tube fouling, thus reducing the efficiency of the thermoelectric power plant. Hard water also causes similar fouling problems associated with calcium deposits in other industries (such as petroleum, chemical, food, agricultural, desalination, etc.) and airconditioning equipment.

Essentially, the present work attempted to develop a mechanical softener with which one could convert hard water to soft water without the use of chemicals and without having to 
replace expensive filter membrane. The present water treatment technology using spark discharges could also replace the ion exchange systems often used at homes in hard water regions. Since the ion exchange units release sodium ions into freshwater resource, the present work could make a major improvement in protecting water resources by stopping the release of sodium ions. The present technology could also prevent or minimize fouling problems in condenser tubes of a desalination process.

Furthermore, the spark discharge in water produces a number of active chemical species including ozone, singlet oxygen, hydrogen peroxide, hydroxyl and superoxide free radicals, ultraviolet radiation (VUV and UV) generated by the discharge and shock waves (Fridman and Kennedy, 2004). Thus, the spark discharge technology can sterilize bio-contaminated wastewater, opening a new opportunity to treat various impaired water for cooling water. In summary, the present spark discharge technology could provide a total solution to cooling water problems, taking care of both mineral scale problem caused by $\mathrm{CaCO}_{3}$ and biofouling problem caused by algae and other microorganisms, while doubling the COC from the current standard value. 


\section{Chapter 2 TECHNOLOGY DESCRIPTION}

\subsection{Objectives of the Project}

The overall objective of the present work was to develop scale-prevention technology using plasma spark discharge by precipitating and removing dissolved mineral ions (such as calcium and magnesium) in cooling water while the $\mathrm{COC}$ was doubled from the present operational value of 3.5 .

\subsection{Scope of the Project}

The present study utilized spark discharges in water to precipitate dissolved mineral ions in recirculating cooling water in a simulated laboratory cooling tower and continuously remove precipitated mineral salts using a self-cleaning filter. We applied a high-voltage pulse $(\sim 40,000$ V) for a very short period (e.g., 10 - 50 nanoseconds) to an electrode immersed in water to produce spark discharges at a frequency of $30-1,000 \mathrm{~Hz}$. The field strength at the tip of the electrode was approximately $1,000,000 \mathrm{~V} / \mathrm{cm}$. The spark discharge also produced a very intense local heating around the tip of the electrode. The local heating could dissociate the bicarbonate ions producing $\mathrm{OH}^{-}$ions, a process which was a critical step in the precipitation reaction of calcium carbonate because the dissociation of $\mathrm{HCO}_{3}^{-}$was the only endothermic process with a positive Gibbs free energy.

More specifically, in Task 1, we conducted validation study to test whether or not the spark discharge could precipitate dissolved calcium ions by measuring the concentration of dissolved calcium ions in cooling water using a calcium ion probe. In Task 2, we conducted tests in a simulated laboratory cooling tower to check if the present technology could continuously precipitate calcium ions and at the same time the self-cleaning filter could continuous remove them such that the blowdown could be eliminated or significantly reduced. We used both calcium probe and electric conductivity meter to monitor the COC level in the recirculating cooling water. In addition, chemical water analysis data (i.e., chloride ion concentration) was measured to confirm the COC calculation. In Task 3, we conducted a series of heat transfer fouling tests using a condenser heat exchanger in the laboratory cooling tower. The fouling resistance was experimentally determined by measuring the inlet and outlet temperatures at both cooling-water side and hot-fluid side. The fouling resistance obtained with the present scale-prevention technology was compared with the no-treatment case as well as the scale-free case.

In summary, we could successfully precipitate and remove the excess calcium ions in cooling water. We could prevent condenser-tube fouling and at the same time double the COC, accomplishing one of the major DOE's goals of reducing/minimizing freshwater withdrawal in thermoelectric power plants. The present study began with a basic scientific research to better understand the mechanism of pulse spark discharges in water and conducted a series of validation experiments using hard water in a laboratory cooling tower. With the completion of the present work, a new prototype hardware using pulse spark discharges became available for 
field validation tests. The plasma water treatment technology developed in the present project is a true mechanical water softener, which continuously converts hard water to soft water with a very little energy consumption.

\subsection{Description of the Project}

\subsubsection{Task 1 - Precipitation of dissolved calcium ions using spark discharge (Year 1)}

One of the primary reasons why thermoelectric generation accounted for 39\% (136 billion gallons per day [BGD)) of all freshwater withdrawals in the nation in 2000 [1) is that the concentration of the mineral ions such as calcium and magnesium in the circulating cooling water increases with time as pure water evaporates to remove heat from condenser tubes. In order to maintain a COC of 3.5 in the cooling water to avoid mineral fouling problem, one needs to continuously blowdown cooling water and replace it with makeup water.

In Task 1 we investigated if we could precipitate and remove the dissolved calcium ions with spark discharges such that blowdown can be eliminated or significantly reduced in cooling water. The precipitation of excess calcium ions in cooling water depended on a number of parameters. In the water side, the $\mathrm{pH}$, the level of supersaturation, and its temperature could affect the precipitation rate of dissolved calcium ions. In the power supply side, the frequency $(\mathrm{Hz})$, power level (voltage), pulse duration (nanosecond), and the rising time of pulse affect the rate of precipitation. In Task 1 , we conducted a study to examine the effects of these variables on the precipitation process of excess calcium ions in cooling water by modeling and experimental study.

\subsubsection{Task 2 - Validation experiments to increase COC (Year 2)}

In Task 2, we investigated if the present technology could increase the COC through a continuous precipitation of calcium ions and removing them with the self-cleaning filter. In Task 2, we built a laboratory cooling tower, where pure water continuously evaporated as we added heat through a small heat exchanger. In the laboratory tower, the amount of water lost by evaporation, wind, and blowdown was automatically replaced by a makeup water valve, which was a floating valve located at the tower sump. The cooling tower had an automatic blowdown capability with a solenoid valve which was turned on-off by a preset conductivity meter. We simulated a typical cooling tower operation using the tap water supplied by the City of Philadelphia. We successfully demonstrated that we could increase COC above 3.5 in cooling water.

\subsubsection{Task 3 - Validation experiments for scale prevention (Year 3)}

In Task 3, we investigated if mineral scale could be prevented or minimized at condenser tubes with a COC of 8 or zero blowdown. We conducted a series of heat transfer fouling tests using a condenser heat exchanger in the laboratory cooling tower. The fouling resistance was 
experimentally determined by measuring the inlet and outlet temperatures at both cooling-water side and hot-fluid side. The fouling resistance obtained with the present scale-prevention technology was compared with the no-treatment case as well as the scale-free case. We could successfully demonstrated that the mineral fouling in condenser tubes could significantly mitigated even in the cases of COC of 8 as well as no blowdown.

In addition, we have conducted tests to check if spark discharges could kill microorganisms in water. In inactivation tests with $E$. coli in water, an approximately $3.5-\log$ reduction in bacterial concentration was obtained after applying a total of 100 pulses to water having a high $E$. coli concentration of $10^{8.5} \mathrm{CFU} / \mathrm{ml}$ of water. The ability of spark discharge technology to sterilize bio-contaminated wastewater opens a new opportunity to treat various impaired water for cooling water. 


\section{TECHNICAL PERFORMANCE}

This section reports the results obtained from the present project.

\section{Chapter 3 OVERVIEW ON PLASMA DISCHARGE AND COOLING WATER TREATMENT}

The term plasma is often referred to as the fourth state of matter in which a certain portion of particles are ionized. The term "plasma" was first introduced by Irving Langmuir, as the way the electrified fluid carried high velocity electrons and ions reminded him of the way blood plasma carried red and white corpuscles. In his paper published in Proceedings of National Sciences of Academy in 1928, he wrote:

"Except near the electrodes, where there are sheaths containing very few electrons, the ionized gas contains ions and electrons in about equal numbers so that the resultant space charge is very small. We shall use the name plasma to describe this region containing balanced charges of ions and electrons."

The ionization of the neutral particles is usually achieved through heating. As temperature rises, molecules become more energetic and transform in the sequence from solid to liquid, gas, and a plasma state. In the plasma state, freely moving particles, including electrons and positively or negatively charged ions, make them electrically conductive, and can attain electrical conductivities sometimes larger than those of metals such as gold and copper.

\subsection{Plasma Generation in Nature and in Laboratory}

Plasmas comprise the majority of the matters in the universe. Most of the stars are made of plasma. The space between the stars is filled with plasma, although at a much lower density than that inside the stars. On the Earth, however, naturally occurring plasma is somewhat rare. In the Earth's atmosphere, the best known plasma phenomenon is the lightening. At an altitude of approximately $100 \mathrm{~km}$, the atmosphere no longer remains non-conducting due to the ionization of neutral molecules by solar radiation, making this region of the atmosphere in a plasma state called "ionosphere". The long-distance communication is largely made possible by the presence of ionosphere through the reflection of radio waves by the ionized layer. Aurora is another example of natural plasma on Earth (see Figure 3-1). At near-space altitudes, the Earth's magnetic field interacts with charged particles from the Sun. These particles are diverted and often trapped by the magnetic field. These trapped particles are most dense near the Poles, causing ionizations of neutral particles in the atmosphere and thus accounting for the light emission of the aurora. 


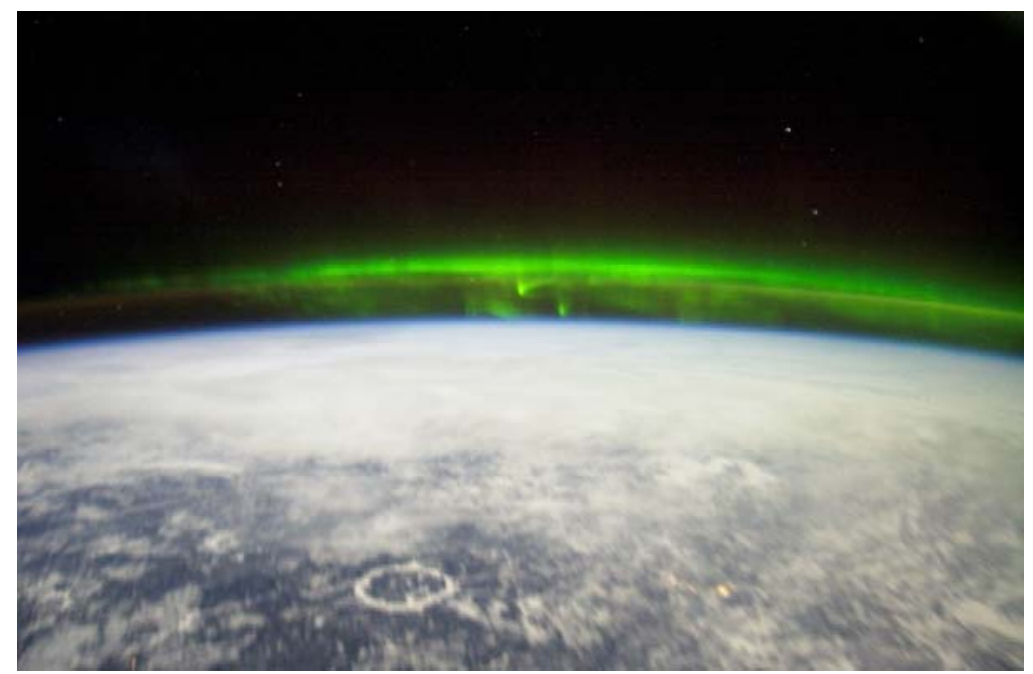

Figure 3-1. Aurora borealis. (NASA)

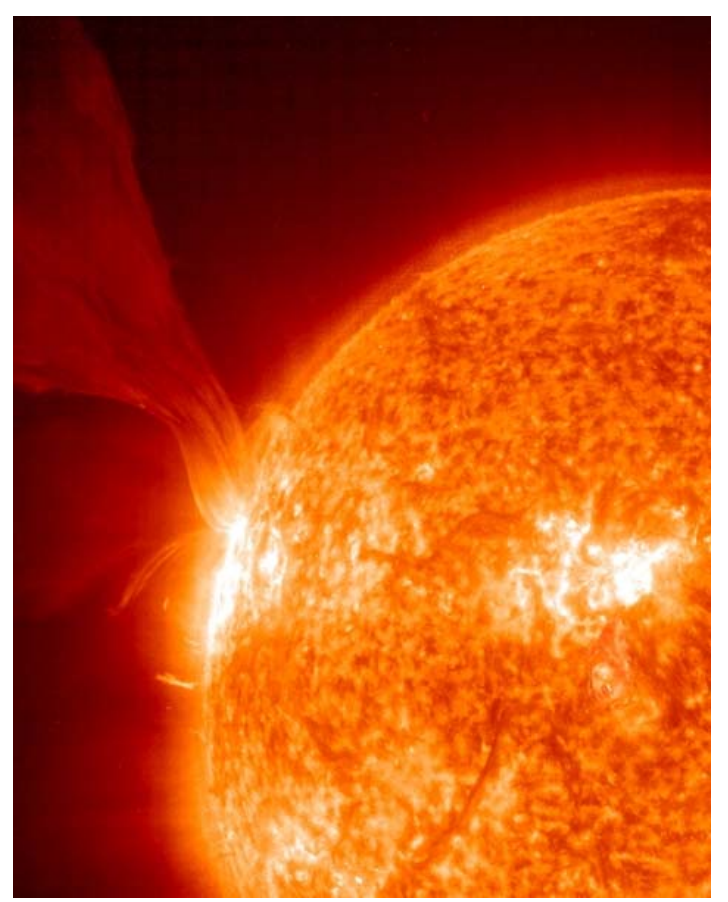

Figure 3-2. Solar plasma. (NASA)

Although the presence of natural plasma on earth is relatively scarce (Figure 3.2), the number of industrial applications of plasma technologies is extensive. Historically, the study of vacuum tubes and so-called "cathode rays" laid the initial foundation of much of our understanding of plasma, which led to the development of plasma lighting technologies since the 19th century. Different energy efficient fluorescent lamps have been available on the market for the past few decades. In recent years, high output RF-powered lamps are being developed as 
a viable alternative to LED (light-emitting diode) lamps, whose manufacturing process also heavily relies on plasma technologies.

Another important application of plasma resides in the semiconductor manufacturing industry. Basically, microelectronics industry would virtually be impossible without plasma, since most processes in the semiconductor device fabrication, including dry etching, deposition and implantation, cannot be achieved by any other commercial method but the plasma.

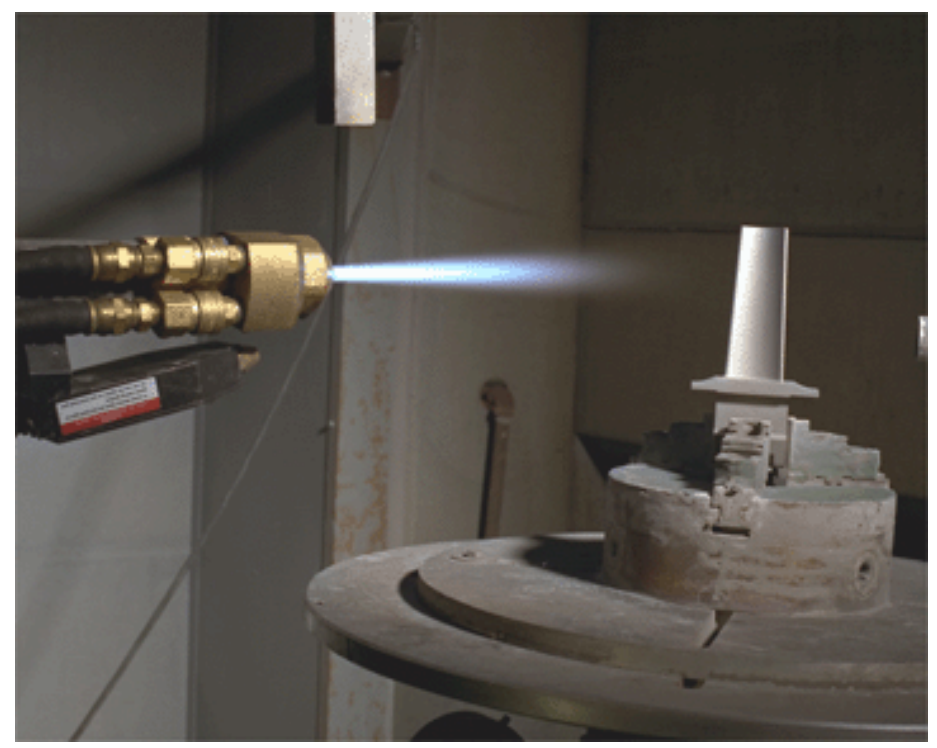

Figure 3-3. Plasma thermal spray coating. (NASA)

Plasma is widely employed in the coating industry where its large enthalpy content, high temperature and high deposition rates are advantageous for increased throughputs. Various materials, including plastics, complex alloys, composites, and ceramics can be deposited over a large area at different shapes. In plasma spraying process (see Figure 3-3), the material to be deposited - typically in a powder form - is introduced into a plasma jet with temperature in the order of $10,000 \mathrm{~K}$. The material is melted and accelerated towards the substrate surface, where the molten droplets rapidly solidify and form the deposition layer.

Surface property modifications for different polymer materials are usually performed using plasma. Many common polymer surfaces are chemically inert, and therefore, pose challenges for use as substrates for applied layers. The modification of polymer surfaces by plasma treatment can improve surface characteristics such as adhesion promotion, enhancing wettability and spreading, improving biocompatibility, functionalizing surface, reducing surface friction and tackiness. These unique surface modifications that can be achieved using the plasma process result from the effects of the photons and active species in the plasma to react with surfaces in depths from several hundred angstroms to microns without influencing bulk properties of the polymer base material. 
Low-temperature, non-equilibrium plasmas are an emerging technology for abating volatile organic compound (VOC) emissions and other industrial exhausts, which have become an important social concern as most of them are carcinogens and harmful to living organisms. Abatement of these polluting substances is conventionally handled by water scrubbers or adsorbent filters to convert them to harmless products. However, for the abatement of diluted VOCs with low concentrations $(<100 \mathrm{ppm})$, these conventional techniques are not suitable mainly due to high-energy consumption. Among the alternatives, non-equilibrium atmospheric pressure plasma processes have been shown to be effective in treating a wide range of emissions including aliphatic hydrocarbons, chlorofluorocarbons, methyl cyanide, phosgene, as well as sulfur and nitrogen oxides. The reduction of the power consumption relies on the selective production of reactive species like ions, radicals and activated molecules by the plasma process without heating of the bulk volume.

\subsection{Needs for Plasma Water Treatment}

The availability of clean water is an issue that has paralleled the continual increase in water consumption due to both global population growth and the economic development in a number of developing countries. From a global perspective, an estimated 2.6 billion people are unable to acquire clean safe drinking water. The global picture shows great disparities between regions (Figure 3-4). Virtually the entire population of the developed regions uses improved facilities for water supply and discharge, but in developing regions only around half the population use improved sanitation facilities.

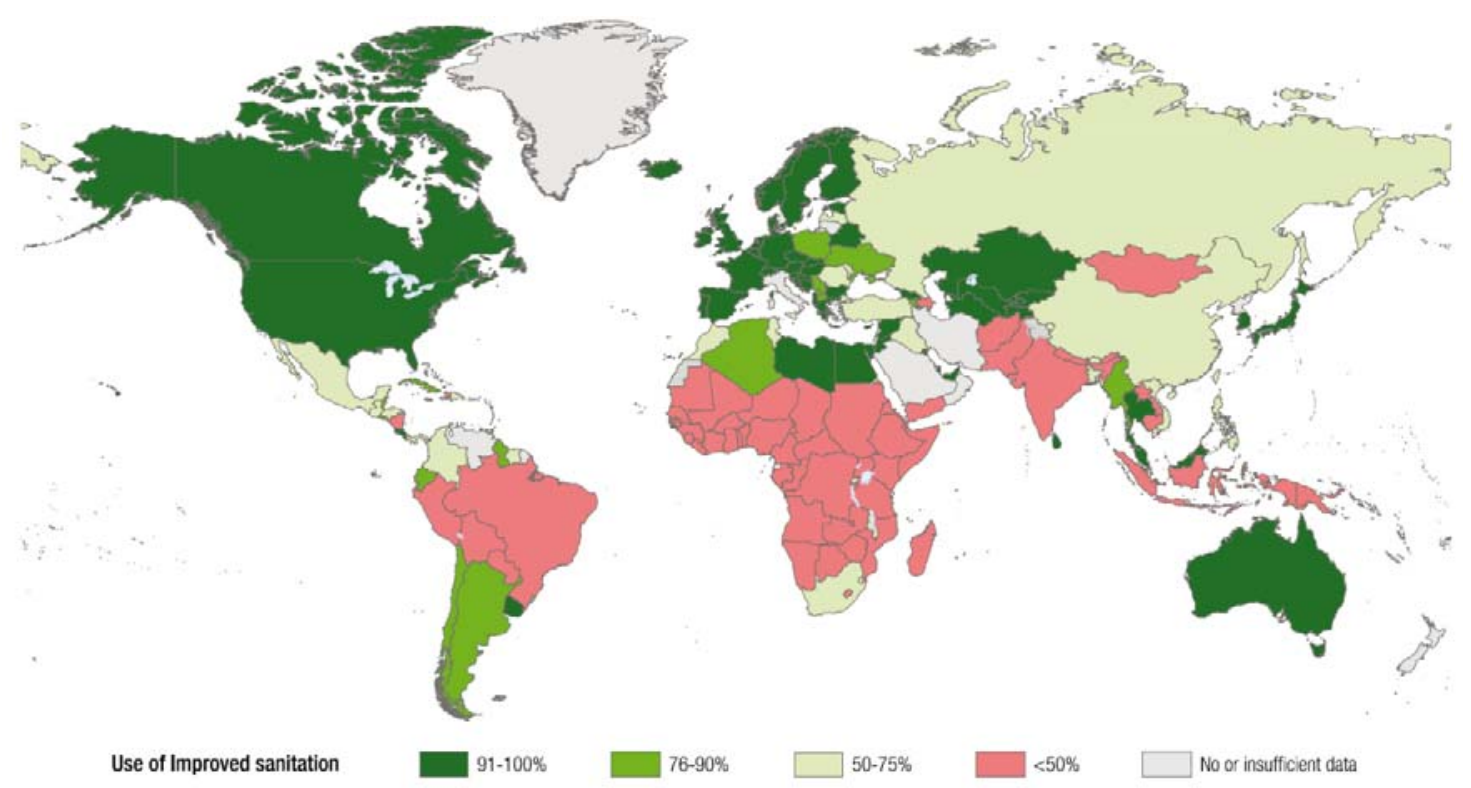

Figure 3-4. Use of sanitary water in rural areas, 2008. (World Health Organization, 2010) 
Contaminated water can be attributed to a number of factors, including chemical fouling, inadequate treatment, and deficient or failing water treatment and distribution system. An additional important cause of the contamination is the presence of untreated bacteria and viruses within the water. The United Nations World Health Organization (WHO) estimated that nearly $35 \%$ of all deaths in developing countries were related directly to contaminated water (World Health Organization, 2010). In United States, the increased presence of Escherichia coli (E. coli) along with various other bacteria within some areas has also become a cause for national concern. In 2006, there was an outbreak of disease caused by the E. coli in 25 U.S. states which caused thousands of illnesses and three deaths. In 2010, more than 500 million eggs were recalled after dangerous levels of Salmonella were detected. In an effort to inactivate these bacteria, traditional chemical treatments, UV radiation, and ozone injection units have been tested and implemented for potable water delivery systems. The experimental success and commercialization of these water treatment methods are not, however, without deficiencies. With regard to human consumption of water, chemical treatments such as chlorination can render potable water toxic. Both UV radiation and ozone injection have been proven to be practical methods of bacterial inactivation in water, but the effectiveness of such methods largely depends upon adherence to regimented maintenance schedules.

Plasma methods effectively combining the contribution of UV radiation, active chemicals, and high electric fields are considered, therefore, as an alternative to these conventional water treatment methods (Locke et al., 2006; Fridman et al., 2007). Before considering direct application of plasma to water treatment (which is a major goal of this section), we will discuss briefly the independent application of UV radiation, active chemicals, and high electric fields for the deactivation of micro-organisms in water.

\subsection{Conventional Water Treatment Technologies}

Currently, there are many available methods of water treatment and decontamination, including chlorination, ozonation, UV radiation, in-line filters and pulsed electric fields. Many of these systems are utilized in large industrial applications. However, methods such as chlorination, in-line filtering and UV radiation are also applied in point-of-use applications, including treatment of swimming pool and well water. These methods have distinct advantages and disadvantages and were carefully analyzed and considered below.

\subsubsection{Chlorination}

The technique of purification of water by use of chlorine was first proposed in early $1800 \mathrm{~s}$. For the past 200 years, chlorine remains both an acceptable and widely employed method of treatment with regard to water disinfection due to its ease of use and associated efficiency for the inactivation of microorganisms. Regardless of system size, it is one of the least expensive disinfection methods. However, the chlorination of public drinking water supplies are meeting with stronger resistance, and people are more concerned about the health effects of the process as the toxicity of chlorine requires strict adherence to accepted concentration levels. An excess 
of chlorine in a drinking water supply could render the water toxic with regard to human ingestion. Unwanted disinfection byproducts (DBPs) resulting from the interaction of chlorine with other chemicals present in water can prove corrosive and deteriorative to the system. Under some circumstances, chlorine can react with organic compounds found in the water supply to produce trihalomethanes (THMs) and haloacetic acids (HAAs), both of which are highly carcinogenic. In addition, because a chlorination based system must be continually replenished, the storage and transportation of this chemical becomes a significant hazard.

\subsubsection{In-line Filters}

In-line filters are commonly used to remove undesirable substances from water. Many different types are commercially available, including activated carbon filters, microfilters and reverse osmosis filters. The key advantage to these filters is that they require non power to operate, but there are two significant drawbacks to this method. Though these filters are capable of preventing microorganisms from passing through the system, they are incapable of inactivating them, leading to bacterial growth in the filters. The small pores needed to trap microorganisms also inhibit the flow, resulting in pressure loss across the filter. Significant pressure losses in the system require larger pump selection.

\subsubsection{Pulsed Electric Field}

The next method considered for inactivating microorganisms is the pulsed electric field technology. Since the electric field associated with this technology is not strong enough (membrane potential of more than $1 \mathrm{~V}$ can kill a bacterium) to initiate electrical breakdown in water, there is no resulting electric discharge. The deactivation of micro-organisms is believed to be due to electroporation, a process which is the creation of holes in cell membranes. It means that plasma-originated electric fields (for example, those in DBD streamers) might be sufficient. At nominal conditions, the energy expense for a two-log reduction is approximately 30,000 J/L (Katsuki et al., 2002). Researchers at the Technical University of Hamburg, Germany reported pulsed electric field effects on suspensions of bacteria in water (Gahl and Markl, 1996). They reported that the external electric pulse produced a membrane potential of more than $1 \mathrm{~V}$ for the effective killing of bacteria.

\subsubsection{Ultraviolet Radiation}

Ultraviolet (UV) radiation has proven to be effective in decontamination processes and is gaining popularity, particularly in European countries, because chlorination leaves undesirable byproducts in water. Measurement of this radiation is considered in terms of dosage, and is the product of intensity $\left(\mathrm{W} / \mathrm{cm}^{2}\right)$ and contact time $(\mathrm{s})$. Most bacteria and viruses require relatively low UV dosages for inactivation, which is usually in a range of $2,000-6,000 \mu \mathrm{W} \cdot \mathrm{s} / \mathrm{cm}^{2}$ for a $90 \%$ kill rate. For example, E. coli requires a dosage of $3,000 \mu \mathrm{W} \cdot \mathrm{s} / \mathrm{cm}^{2}$ for a $90 \%$ reduction (Wolfe, 1990). Cryptosporidium, which shows an extreme resistance to chlorine, requires a UV dosage greater than $82,000 \mu \mathrm{W} \cdot \mathrm{s} / \mathrm{cm}^{2}$. The criteria for the acceptability of UV disinfecting units 
include a minimum dosage of $16,000 \mu \mathrm{W} \cdot \mathrm{s} / \mathrm{cm}^{2}$ and a maximum water penetration depth of approximately $7.5 \mathrm{~cm}$.

UV radiation in the wavelength range from 240 to $280 \mathrm{~nm}$ causes irreparable damage to the nucleic acid of micro-organisms. The most potent wavelength of UV radiation for DNA damage is approximately $260 \mathrm{~nm}$. Currently there are two types of commercial UV lamps: lowpressure and medium-pressure mercury lamps. It is worth noting that the UV radiation from the both types of UV lamp is generated in a plasma environment. The former possesses a relative low temperature and produces a narrow band of UV light with a peak near the 254-nm wavelength, whereas the temperature of the latter is higher and it produces a broader band of UV and has a much greater treatment capacity, approximately 25 times higher than the former (Wolfe, 1990). The life a UV lamp is relatively short, approximately 8,000-10,000 h compromised by several additional factors, including biological shielding and chemical or biological film buildup on the lamp. An advantage of this system is that the temperature and $\mathrm{pH}$ of the treated water are not significantly affected and no undesirable products are created (Wolfe, 1990). However, the total energy cost of the UV water treatment is quite large, similar to the case of the aforementioned pulsed electric fields.

The UV photons can have two possible effects on a microorganism. One effect is through direct collision with the contaminant causing mutation of the bacterial DNA. This prevents proper cellular reproduction and effectively inactivates the microorganism. Alternatively, the photons can provide the necessary energy to ionize or dissociate water molecules, thus generating active chemical species. Both mechanisms increase deactivation of viable microorganisms (Sun et al., 2000). Recently, it is suggested that the UV system produces charged particles in water such that charge accumulation occurs on the outer surface of the bacterial cell membrane. Subsequently, the electrostatic force overcomes the tensile strength of the cell membrane, causing its rupture at a point of small local curvature as the electrostatic force is inversely proportional to the local radius square. Note that since the membrane of gramnegative bacteria such as $E$. coli often possesses irregular surfaces, UV disinfection becomes more effective to the gram-negative bacteria than to gram-positive one (Laroussi, 2005; Laroussi et al., 2002; Hurst et al., 2005).

Researchers at Macquarie University, Australia studied new ultraviolet light sources for the disinfection of drinking water and recycled wastewater (Carman et al., 2003). They reported that UV lamps were much more effective than chlorine in dealing with the hundreds of potentially dangerous types of microbes in water, including the well-known Giardia and Cryptosporidium. The UV radiation did not blow the microbe apart as such. Instead, it entered through the outer membrane of the bacterial into the nucleus and actually cut the bonds of the DNA so that the bacteria could not repair themselves and could not reproduce.

\subsubsection{Ozonation}

Ozonation is a growing method of water treatment, where ozone gas is bubbled into a contaminated solution and dissolves in it. The ozone $\left(\mathrm{O}_{3}\right)$ is one of the most well-known active 
chemical species and is capable of efficiently inactivating microorganisms at a level comparable to chlorine. The existence time of the ozone molecules in the solution depends on temperature. At high temperatures ozone decomposition to molecular oxygen is taking place faster. Solutions maintained at lower temperatures tend to have faster deactivation times when compared to solutions maintained at higher temperatures. Achieving a four-log reduction at $20^{\circ} \mathrm{C}$ with an ozone concentration of $0.16 \mathrm{mg} / \mathrm{L}$ requires an exposure time of $0.1 \mathrm{~min}$ (Anpilov et al., 2001). At higher temperatures and $\mathrm{pH}$ levels, ozone tends to rapidly decay and requires more exposure time. Due to the corrosive and toxic nature of ozone, ozonation systems require a high level of maintenance.

Plasma discharge, especially dielectric barrier discharges (DBD), has been used for the production of ozone in the past several decades to kill microorganisms in water. Ozone has a lifetime of approximately $10-60 \mathrm{~min}$, which varies depending on pressure, temperature and humidity of surrounding conditions. Because of the relatively long lifetime of ozone, ozone gas is produced in air or oxygen, stored in a tank and injected into water using a compressor. Of note is that hydrogen peroxide is also produced when ozone is produced in a plasma discharge in humid air. However, the half life of the hydrogen peroxide is much shorter so that it could not be used for conventional water treatment systems.

The feasibility of using ozonation also was tested for the ballast water treatment for large ships. Drasund et al. reported $C t$ values for various organisms (2001). Note that the $C t$ value is defined as the product of ozone concentration $C(\mathrm{mg} / \mathrm{L})$ and the required contact time $t(\mathrm{~min})$ to disinfect a microorganism in water. For example, for Ditylum brightwelli - important ballast water species, the $\mathrm{Ct}$ value was $50 \mathrm{mg} \cdot \mathrm{min} / \mathrm{L}$. In other words, if the ozone concentration is 2 $\mathrm{mg} / \mathrm{L}$, it takes $25 \mathrm{~min}$ of contact time to disinfect this organism in ballast water. They reported that ozone reacted with seawater and produced a number of corrosive compounds (mostly compounds of chlorine). The long contact time between ozone and organisms is beneficial for the disinfection of organisms but harmful in the corrosion of ballast tank. However, the half life of ozone is relatively short such that the corrosion threat may not last very long. One of the reasons why the ozone has not been used widely in the water treatment system in the U.S. is the relatively high cost of producing ozone, which requires dry air or concentrated oxygen supply, compressor, ozone gas injection system and electricity. Furthermore, if ozone gas is accumulated in a closed space by accident it can be highly toxic to human. In addition, the energy efficiency of ozonation is limited by $\mathrm{O}_{3}$ losses during storage and transportation.

In summary, ozone and UV radiation generated in remote plasma sources are effective means of water cleaning and sterilization. If plasma is organized not remotely but directly in water, the effectiveness of the treatment due to plasma-generated UV radiation and active chemicals can be much higher. The organization of plasma inside water also leads to an additional significant contribution of short-living active species (electronically excited molecules, active radicals like $\mathrm{OH}, \mathrm{O}$, etc.), charged particles, and plasma-related strong electric fields into cleaning and sterilization (Sun, Sato, \& Clements, 1997; Locke et al., 2006; Fridman, Gutsol, \& Cho, 2007). While direct water treatment by plasma generated in the water can be 
very effective, both the initiation and sustaining of plasma in water (where the mean free path of electrons is very short) are more complicated than in the gas phase, a subject which is to be discussed in the following sections.

\subsection{Plasma in Liquids}

Historically, plasmas in liquids were studied by the electrical engineering community for pulsed power applications and high voltage insulation. Liquids, particularly water, usually have higher dielectric constant and higher dielectric strength than their gas phases, and have been widely used as an insulating media for high voltage pulse forming lines in pulse power systems. For example, high-molecular-weight hydrocarbons are frequently used in liquid-filled transformers for both insulation and cooling purposes. The conduction or insulation behavior of liquid is determined by the Maxwellian relaxation time, which is the ratio of dielectric permittivity and electric conductivity. Pure water has a relative dielectric constant $\varepsilon_{\mathrm{r}}$ of 80 up into the $\mathrm{GHz}$ range, and it electrical conductivity $\sigma$ is usually a few $\mu \mathrm{S} / \mathrm{cm}$, resulting in its Maxwellian relaxation time in the order of a few microseconds. Given that a specific water is exposed to an electric pulse with a long duration time of $\Delta \mathrm{t}$, i.e. when $\Delta \mathrm{t} \gg \varepsilon_{\mathrm{r}} \varepsilon_{0} / \sigma$, where $\varepsilon_{0}$ is vacuum permittivity, the aqueous solution behaves as a resistive medium.

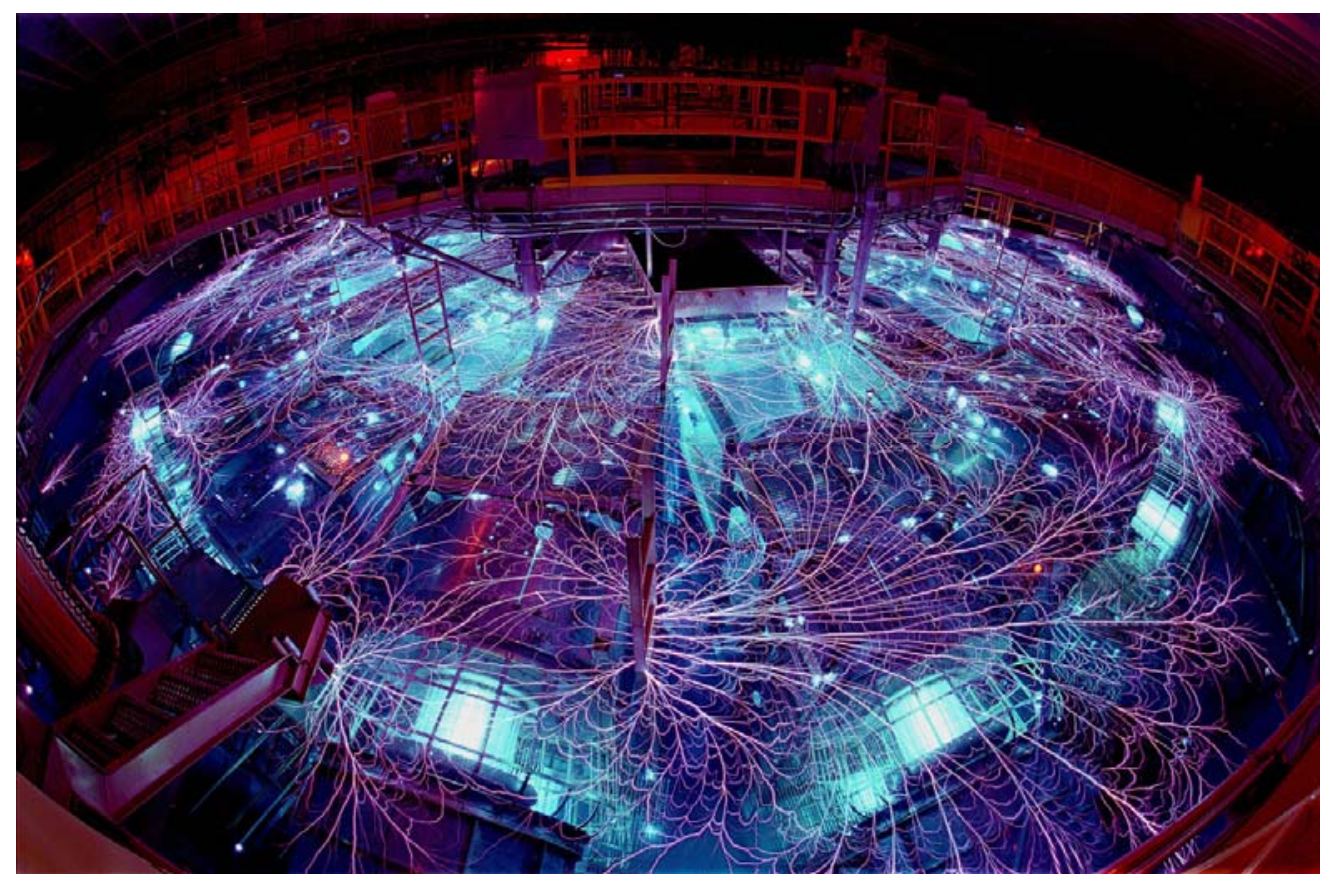

Figure 3-5. Sandia National Lab's Z Machine, bathed in water for greater electric insulation (Sandia National Lab, 2004) 
One of the major results of such a long electric pulse is the electrolysis of water with hydrogen and oxygen production. For much shorter times, i.e. when $\Delta \mathrm{t}<<\varepsilon_{\mathrm{r}} \varepsilon_{0} / \sigma$, water behaves as a dielectric medium, and can sustain a high electric stress until the breakdown threshold is reached. Critical electric breakdown fields of MV/cm have been reported (Locke et al., 2006). These numbers indicate that high energy densities could be achieved in water. The high dielectric strength of liquids allows considerably higher currents and reducing the size of high power switches compared to gaseous switches. Besides the advantage of a lower switch inductance, higher recombination and diffusion rate due to the higher density will quickly restore the switching medium. Small switch volumes also allow to remove the liquid quickly after each shot, and to operate them at high repetition rates. However, this type of switches is not without drawbacks, one of which is their shot-to-shot timing-instability (jitter) originating from the stochastic nature of the breakdown processes. This jitter can be significant, making them not suitable for accurate timing and synchronization purposes.

In recent years there are increasing interests in plasma discharge in liquid mostly because of its potential applications for various biological, environmental and medical technologies. For example, electric breakdown is developed as a non-chemical method for bio-fouling removal and contaminants abatement in water, with a potential for extension into a wide range of other water treatment applications. Intense UV radiation, shockwaves, and various chemical products including $\mathrm{OH}, \mathrm{O}, \mathrm{HO}_{2}, \mathrm{H}_{2} \mathrm{O}_{2}$ from the electric breakdown in water are produced. The synergetic effects of these products are believed to produce higher efficiencies than traditional water treatment technologies utilizing each product. Another application of plasma is to use the plasma as an etching tool for the removal of biological tissues. A plasma scalpel in saline liquid is able to etch flesh and clean wounds during surgery, and is close to widespread practical use (Stalder et. al., 2005). Shock waves produced by high-energy plasma discharges inside liquids are used for various other applications including underwater explosion (Akiyama et. al., 2007), rock fragmentation (Bluhm et. al., 2000), and lithotripsy (Sunka, 2001). In all these applications, it is important to understand the mechanism and dynamics of the electric breakdown process in liquids, a subject which has been under investigation for more than 100 years with a number of experimental results reported.

\subsubsection{Mechanisms of Plasma Discharges in Liquids}

Mechanisms of plasma discharges and breakdowns in liquids (specifically in water) can be classified into two groups: the first group considers the breakdown in water as a sequence of a bubble process and an electronic process in these bubbles, while the second group divides the process into a partial discharge and a fully developed discharge such as arc or spark (Akiyama, 2000). According to the approach from the first group, the bubble process starts from a microbubble which is formed by the vaporization of liquid from local heating in the strong electric field region at the tip of an electrode. The bubble grows, and an electrical breakdown subsequently takes place within the bubble. In this case, a cavitation mechanism is suggested to explain the slow bush-like streamers (Beroural, 1993; Beroural et al. 1998). The appearance of 
bright spots is delayed from the onset of the application of high voltage, and the delay time tends to be greater for lower applied voltages. The time lag to water breakdown increases with increasing pressure, supporting the bubble mechanism in a submicrosecond discharge formation in water (Jones \& Kunhardt, 1994, 1995). The time to form the bubbles is about 3-15 ns, depending on the electric field and pressure (Akiyama, 2000). The influence of the water electrical conductivity on this regime of the discharges is small (Akiyama, 2000).

Bulk heating via ionic current does not contribute to the initiation of the breakdown. The power necessary to evaporate the water during streamer propagation can be estimated using the streamer velocity, the size of the streamer, and the heat of vaporization (Lisitsyn et al., 1999). Using a streamer radius of $31.6 \mu \mathrm{m}$, a power of $2.17 \mathrm{~kW}$ was estimated to be released into a single streamer to ensure its propagation in the form of vapor channels. In the case of multiple streamers, the required power can be estimated by multiplying the number of visible streamers to the power calculated for a single streamer. In the electronic process, both electron injection and drift in liquid take place at the cathode, while hole injection through a resonance tunneling mechanism occurs at the anode (Katsuki et al., 2002). In the electronic process, breakdown occurs when an electron makes a suitable number of ionizing collisions in its transit across the breakdown gap.

According to the approach in the second group on the mechanisms of electrical discharges in water, the discharge process is divided into partial electrical discharges and arc or spark discharge (Locke et al., 2006; Sato, Ohgiyama, \& Clements, 1996; Sugiarto, Ohshima, \& Sato, 2002; Sun, Sato, \& Clements, 1999; Sugiarto et al., 2003; Manolache,Shamamian, \& Denes, 2004; Ching et al., 2001; Ching, Colussi, \& Hoffmann, 2003; Robinson, Ham, \& Balaster, 1973; Robinson, 1973). In the partial discharges, the current is mostly transferred by ions. For the case of water with a high electric conductivity, a large discharge current flows, resulting in a shortening of the streamer length due to the faster compensation of the space charge electric fields on the head of the streamer. Subsequently, a higher power density in the channel is obtained, resulting in a higher plasma temperature, a higher UV radiation, and the generation of acoustic waves. In the arc or spark discharge, the current is transferred by electrons. The high current heats a small volume of plasma in the gap between the two electrodes, generating a quasi-thermal plasma. When a high-voltage, high-current discharge takes place between two submerged electrodes, a large part of the energy is consumed in the formation of a thermal plasma channel. This channel emits UV radiation and its expansion against the surrounding water generates intense shockwaves (Sunka et al., 1999).

In 2006, Locke et al. published a comprehensive review on the application of strong electric fields in water and organic liquids with 410 references (Locke et al., 2006). They explained in detail the types of discharges used for water treatment, physics of the discharge and chemical reactions involved in the discharge in water. More recently, Bruggeman and Leys published another review paper on non-thermal plasma in contact with water (Bruggeman \& Leys, 2009). They discussed three different types of plasmas: direct liquid discharges, discharges in gas phase with a liquid electrode, and discharges in bubbles in liquids. A different 
excitation method for each type was discussed individually. In addition, plasma characteristics of the different types of plasma in liquids were discussed. Currently several research groups around the world actively study plasma discharges for water treatment, which will be briefly discussed next.

\subsubsection{Application of Plasma Discharges in Water}

Schoenbach and his colleagues at Old Dominion University have studied the electrical breakdown in water with submillimeter gaps between pin and plane electrodes by using optical and electrical diagnostics with a temporal resolution on the order of one nanosecond (Joshi et al., 2009; Qian et al., 2006; Qian et al., 2005; Schoenbach et al., 2008; Schoenbach et al., 2007). By using a Mach-Zehnder interferometer, the electric field distribution in the prebreakdown phase was determined by means of the Kerr effect, which indicates a change in the refractive index of a material. Values of electric fields in excess of computed electric fields, which reached over 4 $\mathrm{MV} / \mathrm{cm}$ for applied electrical pulses of $20 \mathrm{~ns}$ duration, were recorded at the tip of the pin electrode. The results of this research have found bioelectric applications in the construction of compact pulse power generators.

Locke and his colleagues at Florida State University have qualitatively studied the production of reductive species by pulsed plasma discharge in water using different chemical probes (Locke, Burlica, \& Kirkpatrick, 2006; Mededovic, Finney, \& Locke, 2008; Sahni \& Locke, 2006). They showed that the formation of primary radicals from water decomposition occurred in the discharge zone. The immediate region surrounding the discharge zone was responsible for radical recombination to form products that diffused into bulk water where the radicals participated in bulk phase reactions. The rate of the formation of reductive species in the pulsed streamer discharge increased as the input power to the system increased, offering a possibility that in a mixture of aqueous contaminants some pollutants or a component of certain pollutants could degrade by reductive mechanisms, thereby increasing the degradation efficiency of the process.

Graves and his colleagues at the University of California, Berkeley presented a unique method to inactivate microorganisms in $0.9 \% \mathrm{NaCl}$ solution (i.e., normal saline solution) by means of microplasmas (Sakiyama et al., 2009). They employed E. coli bacteria to investigate the disinfection efficiency of the device. The device consisted of a thin titanium wire covered by a glass tube for insulation except for the tip of the wire and ground electrode. Microbubbles could be formed at both electrodes from the application of an asymmetric high-frequency, high voltage. . Repetitive light emission was observed in the vicinity of the powered electrode. More than $99.5 \%$ of E. coli was deactivated in $180 \mathrm{~s}$.

Sato and his colleagues at Gunma University, Japan studied the environmental and biotechnological applications of high-voltage pulsed discharges in water (Sato and Yasuoka, 2008; Sato, Yasuoka, \& Ishii, 2008; Sato, Yasuoka, \& Ishii, 2010; Sato, 2008, Sato et al., 2008). A pulsed discharge was formed in water by applying a high-voltage pulse in point-to-plane electrode systems. They found that bubbling through a hollow needle electrode made it possible 
to raise the energy efficiency in the decomposition of organic materials by reducing the initial voltage of the discharge. Oxygen gas bubbling was found to be effective for the decomposition because of the forming of active species originating from oxygen gas.

Sunka and other researchers from the Institute of Plasma Physics, Academy of Sciences of the Czech Republic developed a pulsed corona discharge generator in water using porous ceramic-coated rod electrodes (Lukes et al., 2008; Lukes et al., 2009;Sunka et al., 2004). They studied the properties of the ceramic layer and its interaction with the electrolyte, and reported that surface chemistry at the electrolyte/ceramic surface interface was an important factor in generating electrical discharges in water using porous ceramic-coated electrodes. Initiation of the discharge in water using these types of electrodes depended on the surface charge of the ceramic layer in addition to the permittivity and porosity of the ceramic layer. The surface charge could be determined by the polarity of applied voltage, and $\mathrm{pH}$ and the chemical composition of aqueous solution. By applying bipolar high voltage pulses to eliminate possible buildup of an electrical charge on the ceramic surface, a large-volume plasma could be produced in water in the range of kilowatts. 


\section{Chapter 4 GENERATION OF PLASMA IN LIQUID}

As briefly mentioned in the first chapter, when one considers the mechanism of plasma discharge in water, there can be two different approaches: the first approach classifies the electric breakdown in water into partial discharge and a full discharge such as arc or spark, while the second approach divides the breakdown to a bubble process and an electronic process (Akiyama, 2000). In this chapter, we are going to focus on the first approach, while the second approach will be discussed in the following chapter.

\subsection{Partial and Full Discharges in Liquid}

Electrical discharges in liquid are usually divided into partial and full discharges (Sato, Ohgiyama, and Clements, 1996; Sugiarto, Ohshima, and Sato, 2002; Sun, Sato, and Clements, 1999; Sugiarto et al., 2003; Akiyama, 2000; Lisitsyn et al., 1999; Katsuki et al., 2002; Manolache et al., 2001; Manolache, Shamamian, and Denes, 2004; Ching et al., 2001; Ching, Colus, and Hoffmann, 2003; Destailats et al., 2001). If the discharge does not reach the second electrode, it is called a partial discharge (also called pulsed corona discharge, in analogy with the discharges in gases), and branches of such a discharge are called streamers.

The nature of the discharges in liquids and the mechanism of streamer formation are much less understood, and may be completely different from those for discharges in gases. If a streamer reaches the opposite electrode, it makes a conductive channel between the two electrodes and is usually called a full discharge. Furthermore, if the current through the discharge is very high (above $1 \mathrm{kA}$ ), it is called an arc discharge. While an arc discharge is usually continuous, the transient phase of the arc discharge is referred to as a pulsed spark discharge. In the partial discharge the current is transferred by slow ions, producing corona-like discharges, i.e., non-thermal plasma. For a case of high electrical conductivity liquid, a larger discharge current flows, resulting in a shortening of the streamer length due to the faster compensation of the space charge electric fields on the head of the streamer. Subsequently, a higher power density, i.e., a higher plasma density, in the channel is obtained, resulting in a higher plasma temperature, a higher UV radiation, and the generation of acoustic waves. In the full discharges such as arc or spark, the current is transferred by electrons. The high current heats a small volume of plasma in the gap between the two electrodes, generating quasi-thermal plasma, where the temperatures of electrons and heavy particles are almost equal. 


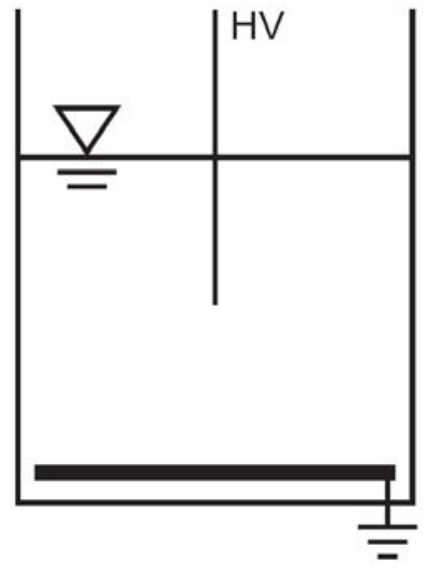

(a)

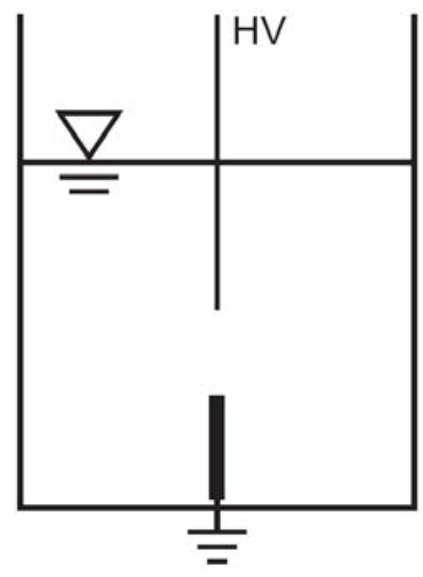

(b)

Figure 4-1. Two electrode configurations for the plasma discharge in water

To generate partial or full electrical discharges in liquid, usually one needs to have a pulsed high voltage power supply with voltage rise time shorter than the Maxwellian relaxation time of the liquid. High electric field strength can usually be achieved by using needle electrodes with sharp tips, from which electric discharges in water usually start. Two simple geometries are shown in Fig. 4-1, which are a point-to-plane geometry and a point-to-point geometry. The characteristics of pulsed corona and pulsed arc are summarized in Table 4-1.
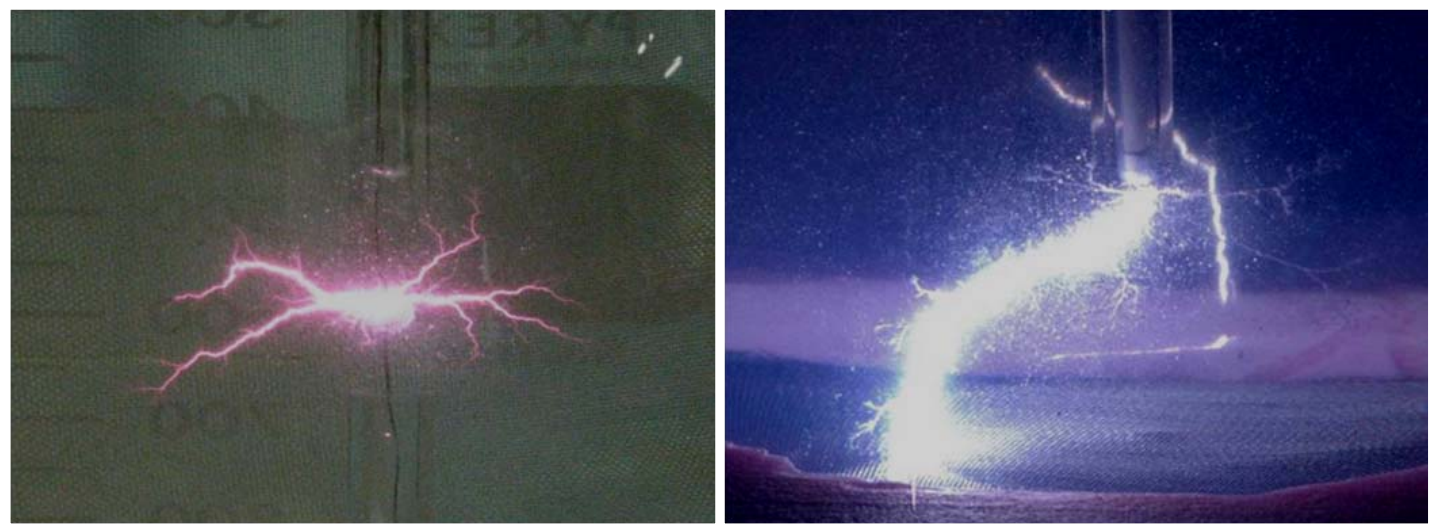

Fig. 4-2. Images of plasma discharge in water: (a) pulsed corona; (b) pulsed arc. 
Table 4-1

Summary of the characteristics of pulsed corona, pulsed arc and pulsed spark discharge in water 1. Pulsed Corona

- Streamer channels do not propagate across the entire electrode gap, i.e., partial electrical discharge

- Streamer length $\sim$ order of centimeters;

- $\quad$ Streamer channel width $\sim 10-20 \mu \mathrm{m}$

- $\quad$ The current is transferred by ions

- $\quad$ Non-thermal plasma

- Weak to moderate UV generation

- Relative weak shockwaves

- Treatment area is limited at a narrow region near the corona discharge

- A few joules per pulse, often less than $1 \mathrm{~J}$ per pulse

- Operating frequency is in a range of $100-1000 \mathrm{~Hz}$

- $\quad$ Relatively low current, i.e., peak current is less than $100 \mathrm{~A}$

- Electric field intensity at the tip of the electrode is $100-10,000 \mathrm{kV} / \mathrm{cm}$

- $\quad$ A fast-rising voltage

2. Pulsed Arc

- The current is transferred by electrons

- Quasi-thermal plasma

- An arc channel generates strong shockwaves within cavitation zone

- High current filament channel bridges the electrode gap

- Channel propagates across the entire electrode gap

- The gas inside channel is ionized

- $\quad$ Strong but short-lived UV emission and high radical density

- A smaller gap between two electrodes of $\sim 5 \mathrm{~mm}$ is needed than that in pulsed corona

- $\quad$ Light pulse from spark discharge includes $\sim 200 \mathrm{~nm}$ wavelength

- Time delay between voltage pulse increase and spark formation depends on both capacitance size and electric conductivity of water

- $\quad$ Large energy discharges greater than $1 \mathrm{~kJ}$ per pulse, desired for wastewater treatment

- $\quad$ Large current on the order of $100 \mathrm{~A}$, with a peak current greater than 1,000 A

- Voltage rise time is in a range of $1-10 \mu \mathrm{s}$

- $\quad$ Pulse duration $\sim 10 \mathrm{~ms}$

- Temperature of the arc is greater than $10,000 \mathrm{~K}$

3. Pulsed Spark

- $\quad$ Similar to pulsed arc, except for shorter pulse duration and lower temperature. 
- $\quad$ Pulsed spark is faster than pulsed arc, i.e., strong shockwaves are produced.

- $\quad$ Plasma temperature in the spark is around a few thousand $\mathrm{K}$.

Although a large number of studies were conducted on plasma discharges in gases, studies on the electric breakdown in liquids have been limited by the high density of liquids and a short mean free path of electrons, therefore requiring a very high electric field $\mathrm{E} / \mathrm{n}_{0}$. The critical breakdown condition for gas can be described by the Paschen curve (Fridman, 2008), from which one can calculate the breakdown voltage. A value of $30 \mathrm{kV} / \mathrm{cm}$ is a well-accepted breakdown voltage of air at $1 \mathrm{~atm}$. When one attempts to produce direct plasma discharge in water, a much higher breakdown voltage on the order of $30 \mathrm{MV} / \mathrm{cm}$ is expected based on the Paschen curve due to the density difference between air and water. A large number of experimental data on the breakdown voltage in water showed, however, that the breakdown voltage in water was higher, but still of the same magnitude as for gases. In other words, the breakdown of liquids can be performed not at the extremely high electric fields required by the Paschen curve but at those that only slightly exceed the breakdown electric fields in atmospheric-pressure molecular gases.

This interesting and practically important effect can be explained by taking into account the fast formation of gas channels in the body of water under the influence of an applied high voltage. When formed, the gas channels give the space necessary for the gas breakdown inside water, explaining why the voltage required for the breakdown in water is of the same magnitude as that in gases. The gas channels can be formed through the development and electric expansion of gas bubbles already existing in water or by additional formation of the vapor channel through fast local heating and evaporation.

\subsubsection{Thermal Breakdown Mechanism}

When a voltage pulse is applied to water, it induces a current and redistribution of the electric field. More specifically, the voltage pulse once applied immediately stimulates the rearrangement of electric charges in water, and the rearrangement of the electric charges results in a fast redistribution of electric field in water. Due to the dielectric nature of water, an electric double layer is formed near the electrode, resulting in the localization of the major portion of the applied electric field in the vicinity of the electrode. This electric field can become high enough for the formation of a narrow conductive channel, which is heated up by electric current to temperatures of about $10,000 \mathrm{~K}$. Thermal plasma generated in the channel is rapidly expanding from the narrow channel into water, forming a plasma bubble. High electric conductivity in the plasma channel leads to the shifting of the high electric fields from the channel to the bubble. These electric fields provide a drift of negatively charged particles from the bubble into the channel. Taking into account that the temperature in the plasma bubble is not large enough to cause thermal ionization, and the electric field at the bubble is not sufficient to cause direct 
electric impact ionization, the oxygen-containing negative ions from water are believed to make major contributions in the negative charge transfer from the bubble into the channel.

The plasma bubble can be characterized by a very high temperature gradient and a large electric field. The energy required to form and sustain the plasma bubble is provided by Joule heating in the narrow conductive channel in water. High current density in the channel is limited by the conductivity in the relatively cold plasma bubble, where temperature is about 2,000 K. The electric conductivity in the bubble is determined not by electrons but by negative oxygencontaining ions. Further expansion of the plasma bubble leads to its cooling and decreasing of the charged particles densities. Subsequently, the electric current decreases, resulting in a significant reduction in Joule heating in the conductive channel in water and eventual cooling down of the channel itself.

The physical nature of thermal breakdown can be related to thermal instability of local leakage currents through water with respect to the Joule overheating. If the leakage current is slightly higher at one point, the Joule heating and, hence, temperature also increase there. The temperature increase results in a significant growth of local electric conductivity and leakage current. Temperature increases exponentially to several thousand degrees at a local point, leading to the formation of the narrow plasma channel in water, which results in the thermal breakdown. The thermal breakdown is a critical thermo-electric phenomenon taking place at the applied voltages exceeding a certain threshold value, when heat release in the conductive channel cannot be compensated by heat transfer losses to the surroundings. The described sequence of plasma channel events takes place in frameworks of a single voltage pulse. When the next voltage pulsed is applied to water, a new thermal breakdown and new micro-arc occur in other surface spot on the electrode.

During the plasma discharge the thermal condition of water is constant. For water relatively away from the discharge, it stays in liquid state with a thermal conductivity about 0.68 $\mathrm{W} / \mathrm{mK}$. When Joule heating between the two electrodes is larger than the aforementioned threshold value, an instability can occur, resulting in instant evaporation and a subsequent thermal breakdown. On the other hand, when Joule heating is smaller than the threshold value, nothing happens but electrolysis, and hence the breakdown never takes place. The Joule heating is inversely proportional to the resistance of matter when a fixed voltage is applied between the two electrodes. The resistance is inversely proportional to the electric conductivity of the dielectric medium (here initially liquid water and later water vapor).

To analyze the thermal instability it can be assumed that electric conductivity of water $\sigma_{\mathrm{e}}$ can be expressed as an exponential function of temperature $\mathrm{T}$ :

$$
\sigma_{e}=\sigma_{0} e^{-\left(E_{a} / R T\right)}
$$

where $\mathrm{E}_{\mathrm{a}}$ is an activation energy, $\sigma_{0}$ is the initial electric conductivity, and $\mathrm{R}$ the universal gas constant. When the medium temperature increases, the electric conductivity of dielectric medium increases, resulting in the decrease in the resistance. Thus, the Joule heating increases, 
increasing the temperature of the dielectric medium. Subsequently, the increased temperature increases the electric conductivity, further increasing temperature, leading to a thermal "explosion" that can be referred to as an instability and descrbied by linear perturbation analysis of the transient energy conservation equation:

$$
\rho C_{p} \frac{\partial T}{\partial t}=\sigma_{0} e^{-\left(\frac{E_{a}}{R T}\right)}\left(E^{2}-k \nabla^{2} T\right)
$$

where $\rho C_{\mathrm{p}}$ is the specific heat per unit volume, $\mathrm{E}(\mathrm{V} / \mathrm{cm})$ is the electric field and $\mathrm{k}$ is the thermal conductivity of water. The second term in the right-hand side represents heat conduction, which takes place with a large temperature gradient along the radial direction. The minus sign means that it represents heat loss to the surrounding water. Note that the convection heat loss is not considered because there is no time for heat to dissipate via convection.

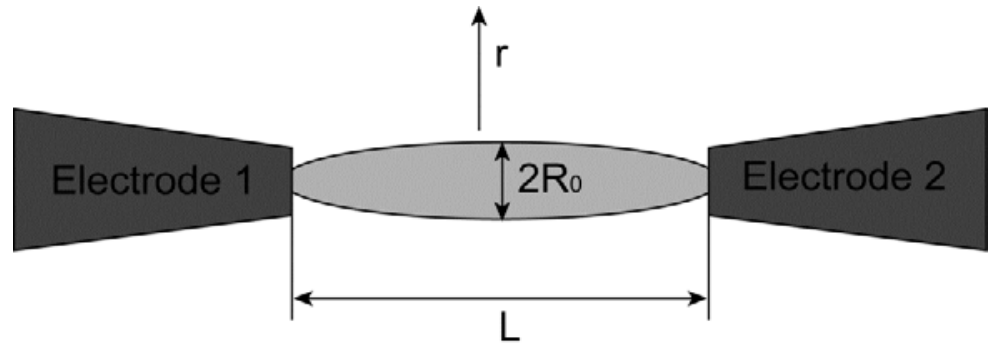

Figure 4-3. Sketch of a plasma channel between two electrodes surrounded by water

The instability is usually described in terms of its increment $\Omega$, which is an angular frequency $(\mathrm{rad} / \mathrm{s})$. When $\Omega$ is greater than zero, the perturbed temperature exponentially increases with time, resulting in thermal explosion. When $\Omega$ is less than zero, the perturbed temperature exponentially decreases with time, resulting in the steady-state condition. When $\Omega$ is complex, the perturbed temperature oscillates with time. The linear perturbation analysis of Equation (4-2) leads to the following expression for the increment of the thermal breakdown instability (Fridman, Gutsol, \& Cho, 2007):

$$
\Omega=\left[\frac{\sigma_{0} E^{2}}{\rho C_{p} T_{0}}\right] \frac{E_{a}}{R T_{0}}-D \frac{1}{R_{0}^{2}}
$$

where $R_{0}$ is the radius of the breakdown channel, and $D \approx 1.5 \cdot 10^{-7} \mathrm{~m}^{2} / \mathrm{s}$ is the thermal diffusivity of water with $\mathrm{C}_{\mathrm{p}}$ of $4179 \mathrm{~J} / \mathrm{kg} \cdot \mathrm{K}$ and $\mathrm{k}$ of $0.6 \mathrm{~W} / \mathrm{m} \cdot \mathrm{K}$. The first term is made up of the product of two, $\left[\sigma_{0} E^{2} / \rho C_{p} T_{0}\right]$ represents the frequency of heating as the numerator is Joule heating, whereas the denominator is the heat stored in the water medium; $\left[E_{a} / R T_{0}\right]$ represents the activation energy to temperature, a sensitivity indicator. The second term in the right-hand side represents the ratio of the thermal diffusivity to the square of the radial characteristic length for radial heat conduction (see Figure 4-1), indicating how fast heat dissipates along the radial 
direction. The first term is only active during the period when the pulse power is on, while the second term is active even during the period of the pulse power turned off. Hence, there is a balance between the Joule heat generation by pulse discharges and heat conduction to the surrounding water. When the heat generation is greater than the conduction loss, the increment $\Omega$ becomes positive, leading to the thermal explosion. Hence, the critical phenomenon leading to the thermal explosion is given as follows:

$$
\left[\frac{\sigma_{0} E^{2}}{\rho C_{p} T_{0}}\right] \frac{E_{a}}{R T_{0}} \geq D \frac{1}{R_{0}^{2}}
$$

Note that $\Omega=0$ means the transition from the stabilization to thermal explosion, a condition that can be defined as the critical phenomenon.

Since the electric conductivity $\sigma$ of a dielectric medium is extremely sensitive to temperature as shown in Equation (4-1), one can expect that as the temperature increases, the breakdown voltage would decrease.

The breakdown voltage $\mathrm{V}$ is given by the product of electric field strength $\mathrm{E}$ and the distance between two electrodes L. Thus, one can rewrite the above equation as:

$$
\left[\frac{\sigma_{0}(E L)^{2}}{\rho C_{p} T_{0}}\right] \frac{E_{a}}{R T_{0}}=\left[\frac{\sigma_{0} V^{2}}{\rho C_{p} T_{0}}\right] \frac{E_{a}}{R T_{0}} \geq D \frac{1}{R_{0}^{2} / L^{2}}
$$

If we introduce a geometry factor, $\mathrm{G}=\mathrm{L} / \mathrm{R}_{0}$, one can rewrite the above equation as:

$$
\left[\frac{\sigma_{0} V^{2}}{\rho C_{p} T_{0}}\right] \frac{E_{a}}{R T_{0}} \geq D G^{2}
$$

From this equation, the breakdown voltage $\mathrm{V}$ can be obtained as:

$$
V \geq \sqrt{\frac{k R T_{0}^{2}}{\sigma_{0} E_{a}}} G
$$

For the plasma discharge in water, the breakdown voltage can be numerically estimated as follows:

$$
V \geq \sqrt{\frac{k R T_{0}^{2}}{\sigma_{0} E_{a}}} G
$$


For the plasma discharge in water, the breakdown voltage can be numerically estimated as follows:

$$
V \geq \sqrt{\frac{k R T_{0}^{2}}{\sigma_{0} E_{a}}} G=\sqrt{\frac{0.613 \times 461.5 \times 300^{2}}{0.05 \times 700}} G \approx 26 G
$$

For a case of $\mathrm{L}=1 \mathrm{~cm}$, the diameter of the streamer is usually in the order of $10 \mu \mathrm{m}$, leading to the geometry factor $\mathrm{G}=\mathrm{L} / \mathrm{R}_{0}=1000$, and the breakdown voltage in water becomes $26,000 \mathrm{~V}$.

\subsubsection{Reactive Species, UV and Shockwave Production by Electrical Discharges in Liquid}

When a plasma discharge is initiated between two electrodes, the medium between the two electrodes is ionized creating a plasma channel. The plasma discharge generates ultraviolet radiation and converts surrounding water molecules into active radical species due to the high energy level produced by the discharge. The microorganisms could be effectively inactivated, while the organic contaminants could be oxidized through the contact with active radicals. The chemical kinetics of these reactions remains an area of significant research. Various active species can be considered as the byproducts of plasma discharge in water. The production of these species by plasma discharge is affected by a number of parameters such as applied voltage, rise time, pulse duration, total energy, polarity, the electric conductivity of water, etc. Among the active species, hydroxyl radical, atomic oxygen, ozone and hydrogen peroxide are the most important ones for the sterilization and removal of unwanted organic compounds in water. Table 4-2 summarizes the oxidation potentials of various active species produced by plasma in water, which ranges from $1.78 \mathrm{~V}$ (hydrogen peroxide) to $2.8 \mathrm{~V}$ (hydroxyl radical). Note that fluorine has the highest oxidation potential of $3.03 \mathrm{~V}$, whereas chlorine, which is one of the most commonly used chemicals for water decontamination, has an oxidation potential of only $1.36 \mathrm{~V}$.

Table 4-2

Oxidation potential of common oxidative agents and active species produced by plasma in water under standard conditions

\begin{tabular}{|c|c|c|}
\hline Chemical & Reaction & Oxidation Potential (V) \\
\hline $\mathrm{O}_{2}$ & $1 / 2 \mathrm{O}_{2}+2 \mathrm{H}^{+}+2 e \rightarrow \mathrm{H}_{2} \mathrm{O}$ & 1.23 \\
\hline $\mathrm{Cl}_{2}$ & $1 / 2 \mathrm{Cl}_{2}+e \rightarrow \mathrm{Cl}^{-}$ & 1.36 \\
\hline $\mathrm{Fe}^{3+}$ & $\mathrm{Fe}^{3+}+e \rightarrow \mathrm{Fe}^{2+}$ & 0.77 \\
\hline $\mathrm{F}_{2}$ & $1 / 2 \mathrm{~F}_{2}+e \rightarrow \mathrm{F}^{-}$ & 3.03 \\
\hline $\mathrm{MnO}_{4}^{-}$ & $\mathrm{MnO}_{4}^{-}+8 \mathrm{H}^{+}+5 e \rightarrow \mathrm{Mn}^{2+}+4 \mathrm{H}_{2} \mathrm{O}$ & 1.51 \\
\hline $\mathrm{O}_{3}$ & $\mathrm{O}_{3}+2 \mathrm{H}^{+}+2 e \rightarrow \mathrm{O}_{2}+\mathrm{H}_{2} \mathrm{O}$ & 2.07 \\
\hline $\mathrm{O}$ & $O+2 \mathrm{H}^{+}+2 e \rightarrow \mathrm{H}_{2} \mathrm{O}$ & 2.42 \\
\hline $\mathrm{OH}$ & $\mathrm{OH}+\mathrm{H}^{+}+e \rightarrow \mathrm{H}_{2} \mathrm{O}$ & 2.81 \\
\hline
\end{tabular}




\begin{tabular}{|c|c|c|}
\hline $\mathrm{H}_{2} \mathrm{O}_{2}$ & $1 / 2 \mathrm{H}_{2} \mathrm{O}_{2}+H^{+}+e \rightarrow \mathrm{H}_{2} \mathrm{O}$ & 1.78 \\
\hline $\mathrm{HO}_{2}$ & $\mathrm{HO}_{2}+H^{+}+e \rightarrow \mathrm{H}_{2} \mathrm{O}_{2}$ & 1.50 \\
\hline $\mathrm{O}_{2}^{-}$ & $\mathrm{O}_{2}^{-}+H^{+} \rightarrow \mathrm{HO}_{2}$ & 1.00 \\
\hline
\end{tabular}

A detailed schematic of the chemistry relative to plasma inside or over water can be found in the recent review by Bruggeman and Leys (2009). Major reactions for radical production are summarized in Table 4-3.

Table 4-3. Major reactions for production of reactive species in liquid plasma

\begin{tabular}{|c|c|c|}
\multicolumn{2}{c}{$\left(\mathrm{T}_{\mathrm{e}} \approx 1 \mathrm{ev}, \mathrm{T}_{\mathrm{g}} \approx 300 \mathrm{~K}\right)$} \\
\hline Reaction & Reaction Rate $\left(\mathrm{m}^{3} \mathrm{~s}^{-1}\right)$ & Reference \\
\hline$e+\mathrm{H}_{2} \mathrm{O} \rightarrow \mathrm{OH}+\mathrm{H}+e$ & $2.3 \times 10^{-18}-1.8 \times 10^{-16}$ & Itikawa et al., 2005 \\
\hline$e+\mathrm{H}_{2} \mathrm{O} \rightarrow \mathrm{OH}+\mathrm{H}^{-}$ & $4.9 \times 10^{-18}-4.7 \times 10^{-17}$ & Itikawa et al., 2005 \\
\hline$e+\mathrm{H}_{3} \mathrm{O}^{+} \rightarrow \mathrm{OH}+\mathrm{H}_{2}$ & $10^{-13}$ & Miller et al., 1997 \\
\hline$e+\mathrm{H}_{2} \mathrm{O}^{+} \rightarrow \mathrm{OH}+\mathrm{H}$ & $2.6 \times 10^{-14}$ & Jensen et al., 1999 \\
\hline $\mathrm{OH}+\mathrm{OH} \rightarrow \mathrm{H}_{2} \mathrm{O}+\mathrm{O}$ & $1.9 \times 10^{-18}$ & Herron and Green, 2001 \\
\hline $\mathrm{OH}+\mathrm{OH} \rightarrow \mathrm{H}_{2} \mathrm{O}_{2}$ & $2.6 \times 10^{-17}$ & Herron and Green, 2001 \\
\hline $\mathrm{H}_{2} \mathrm{O}+\mathrm{H}_{2} \mathrm{O}^{+} \rightarrow \mathrm{OH}+\mathrm{H}_{3} \mathrm{O}^{+}$ & $1.9 \times 10^{-15}$ & Gordillo-Vazquez, 2008 \\
\hline $\mathrm{H}^{-}+\mathrm{H}_{2} \mathrm{O}^{+} \rightarrow \mathrm{OH}+\mathrm{H}_{2}$ & $2.0 \times 10^{-13}$ & Kushner, 1999 \\
\hline $\mathrm{H}^{-}+\mathrm{H}_{3} \mathrm{O}^{+} \rightarrow \mathrm{OH}+\mathrm{H}_{2}+\mathrm{H}$ & $2.3 \times 10^{-13}$ & Miller et al., 1997 \\
\hline $\mathrm{OH}+\mathrm{O} \rightarrow \mathrm{O}_{2}+\mathrm{H}^{\mathrm{H}}$ & $3.3 \times 10^{-17}$ & Herron and Green, 2001 \\
\hline $\mathrm{OH}+\mathrm{H}_{\mathrm{H}} \mathrm{O}$ & $2 \times 10^{-16}$ & Herron and Green, 2001 \\
\hline $\mathrm{OH}+\mathrm{H}_{2} \mathrm{O}_{2} \rightarrow \mathrm{HO}_{2}+\mathrm{H}_{2} \mathrm{O}$ & $1.7 \times 10^{-19}$ & Herron and Green, 2001 \\
\hline
\end{tabular}

In addition to the active species, the electrical breakdown in water produces ultraviolet radiation (both VUV and UV). VUV (i.e., vacuum UV), as the name indicates, can only propagate in vacuum because it is strongly absorbed by air or water due to its high energy. For pulsed arc discharge, the high temperature plasma channel can function as a blackbody radiation source. The maximum emittance is in the UVa to UVc range of the spectrum $(200-400 \mathrm{~nm})$ (Robinson, Ham, and Balaster, 1973; Sunka, 2001), as determined by the Stephen-Boltzmann law. Water is relatively transparent to UV radiation in this wavelength range as long as water remains clear without suspended particles. The energy per photon ranges from $3.1 \mathrm{eV}$ to $6.2 \mathrm{eV}$. UV radiation has proven to be effective for decontamination processes and is gaining popularity as a means for sterilization because chlorination leaves undesirable byproducts in water. The radiation in the wavelength range of $240-280 \mathrm{~nm}$ may cause an irreparable damage to the nucleic acid of microorganisms, preventing proper cellular reproduction, and thus effectively inactivating the microorganisms. 
Table 4-4 Types of ultraviolet radiation

\begin{tabular}{|c|c|c|c|}
\hline Name & Abbreviation & Wavelength $(\mathrm{nm})$ & Energy $(\mathrm{eV})$ \\
\hline Ultraviolet A & UVA & $315-400$ & $3.10-3.94$ \\
\hline Ultraviolet B & UVB & $280-315$ & $3.94-4.43$ \\
\hline Ultraviolet C & UVC & $200-280$ & $4.43-6.20$ \\
\hline Vacuum Ultraviolet & VUV & $100-200$ & $6.20-12.4$ \\
\hline
\end{tabular}

Alternatively, the photons can provide the necessary energy to ionize or dissociate water molecules, generating active chemical species. Recently, it is suggested that the UV system may produces charged particles in water such that charge accumulation occurs on the outer surface of the membrane of bacterial cell (Laroussi et al., 2002). Subsequently, the electrostatic force on the membrane overcomes the tensile strength of the cell membrane, causing its rupture at a point of small local curvature as the electrostatic force is inversely proportional to the local radius squared.

Next the ability for the discharge to generate shockwaves will be briefly summarized. When a high voltage, high current discharge takes place between two electrodes submerged in water, a large part of the energy is consumed on the formation of a thermal plasma channel. The expansion of the channel against the surrounding water generates a shockwave. For the corona discharge in water the shockwaves are often weak or moderate, whereas for the pulsed arc the shockwaves are strong. The difference arises from the fact that the energy input in the arc or spark discharge is much higher than that in the corona.

Similarly, between the arc and spark, the arc produces much greater shockwaves due to its higher energy input than spark. The water surrounding the electrodes becomes rapidly heated, producing bubbles, which help the formation of a plasma channel between the two electrodes. The plasma channel may reach a very high temperature of $14,000-50,000 \mathrm{~K}$, consisting of a highly ionized, high pressure and high temperature gas. Thus, once formed, the plasma channel tends to expand. The energy stored in the plasma channel is dissipated via both radiation and conduction to the surrounding cool liquid water as well as mechanical work. At the liquid-gas phase boundary, the high pressure build-up in the plasma is transmitted into the water interface and an intense compression wave (i.e., shockwave) is formed, traveling at a much greater speed than the speed of sound. Note that the shockwaves have another benefit in the sterilization process through a good mixing of water to be treated, significantly enhancing the plasma treatment efficiency.

However, the plasma discharge for water treatment is not without deficiencies. One of the concerns in the use of a sharp needle as a high-voltage electrode is the adverse effect associated with the needle tip erosion. In a point-to-plane geometry, a large electric field can be achieved due to the sharp tip of the needle with a minimum applied voltage V. For a sharp parabolic tip of the needle electrode, the theoretical electric field at the needle tip becomes $E \propto V / r$, where $\mathrm{r}$ 
is the radius of curvature of the needle tip. As indicated by the above equation, the electric field at the tip of the electrode is inversely proportional to the radius of curvature of the needle tip. Hence, the maximum electric field could be obtained by simply reducing the radius of curvature $\mathrm{r}$, which is much easier than increasing the voltage as the maximum value of the voltage is usually restricted by the electric circuit as well as insulation materials used around electrodes. Sunka et al. (1999) pointed out that the very sharp tip anode would be quickly eroded by the discharge, and one had to find some compromise between the optimum sharp anode construction and its lifetime for extended operation. Also it was demonstrated recently that the erosion of electrodes at pulse electric discharge in water would result in the production of metal and oxide nanoparticles in water (Kolikov et al., 2005; Kolikov et al., 2007). These particles are very difficult to remove once they enter the drinking water system due to their nanometer sizes, and potential danger to human body is not clearly known.

Another concern in the application of pulsed electric discharges in water is the limitation posed by the electrical conductivity of water on the production of such discharges (Sunka et al., 1999). In the case of a low electric conductivity below $10 \mu \mathrm{S} / \mathrm{cm}$, the range of the applied voltage that can produce a corona discharge without sparking is very narrow. On the other, in the case of a high electric conductivity above $400 \mu \mathrm{S} / \mathrm{cm}$, which is the typical conductivity of tap water, streamers become short and the efficiency of radical production decreases. In general, the production of hydroxyl radicals and atomic oxygen is more efficient at water conductivity below $100 \mu \mathrm{S} / \mathrm{cm}$. Thus, this is one of the major challenges in the application of plasma discharges for cooling water management as the electric conductivity of most cooling water is at the range of $2000-4000 \mu \mathrm{S} / \mathrm{cm}$. Even bigger challenges exist for the treatment of seawater (for example ballast water) where the electric conductivity can be higher than $10,000 \mu \mathrm{S} / \mathrm{cm}$.

\subsection{Underwater Plasma Sources}

\subsubsection{Direct Discharges in Liquid}

Various electrode geometries have been studied for the generation of plasma discharges in liquid. Figure 4-3 shows some of the typical electrode configurations. Note that only the cases where both the high voltage electrode and ground electrode are placed in liquid are shown here. In general, electrodes with small curvature radius $r_{c}$ at the tips are used to ignite a plasma discharge, because it can produce a high initiation electric field in liquid. Among them, the point-to-plane geometry has been the most commonly used configuration (shown in Figures 41a and 4-4a). Also a point-to-plane geometry with multiple points was used to generate a larger volume corona discharge in water (Figure 4-4b). For pulsed arc discharges, a point-to-point electrode geometry was often used (Figures $4-1 b$ and $4-4 c$ ). The electric field at the tip of the electrode can be estimated as $U / r_{c}$, where $U$ is the applied voltage, and $r_{c}$ is the radius of curvature at the tip. For a $100 \mu \mathrm{m}$-diameter wire and typical $30 \mathrm{kV}$ voltage for usual point configurations, the enhanced electrical field using a small $r_{c}$ can reach the $M V / c m$ required for most of liquid discharge initiation. 


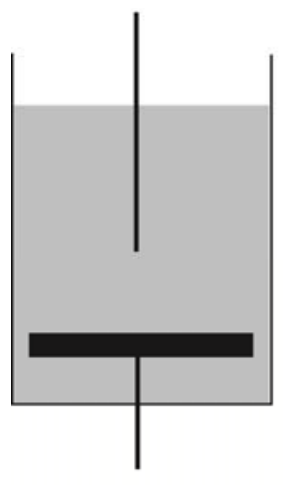

(a)

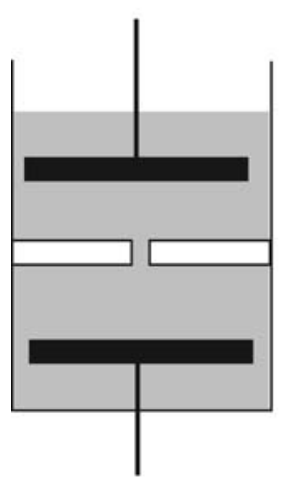

(d)

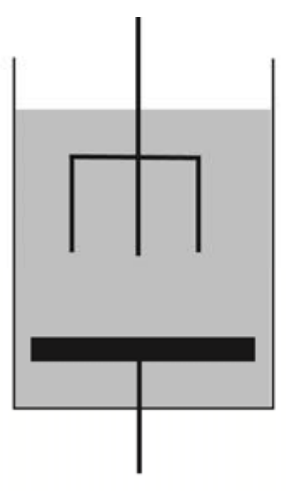

(b)

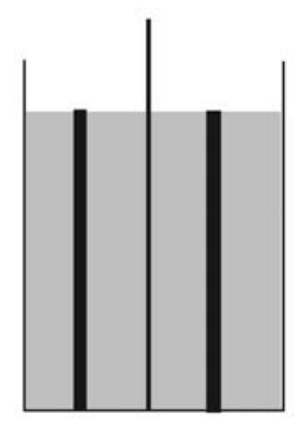

(e)

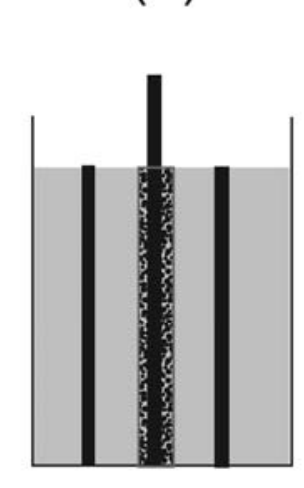

(g)

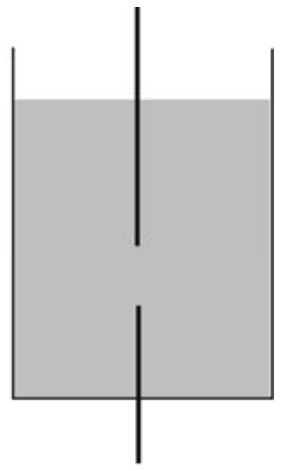

(c)

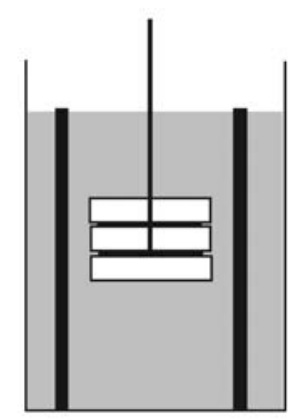

(f)

\section{)}

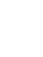

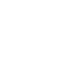


achieve the goal of local electric field enhancement. One example is "pinhole" electrodes (also called a diaphragm discharge, as shown in Fig. 4-4d), where the high voltage and ground electrodes are separated by a dielectric disk with a small hole at the center. The diameter of the pinhole typically varies from 0.1 to $1 \mathrm{~mm}$. When high voltage is applied on the electrodes, an intense electric field could be formed around the pinhole. Subsequently, a pre-discharge current could be concentrated in the small hole, leading to strong thermal effects, resulting in the formation of bubbles and breakdown. Pulsed corona discharge occurs inside the bubbles at the pinhole because of the high electric field. The length of the streamers generated is decided by the parameters such as water conductivity, the size of the pinhole, flow velocity through the pinhole, and voltage polarity. It was observed that when the flow rate through the pinhole was increased, the length of the streamers decreased, possibly due to the flow washing out tiny bubbles or ions from the pinhole (Yamada et al., 1998). Similar to the corona discharge in the point-to-plane geometry, a pulsed arc discharge could be formed once the streamer bridges the two electrodes. Figure 4-5 shows (a) pulsed corona and (b) arc discharges through a pinhole developed at Drexel Plasma Institute. Similar design with multiple pinholes was attempted. It was observed that it was very difficult to discharge simultaneously at each pinhole, and very high overvoltage with short rise time is usually required.

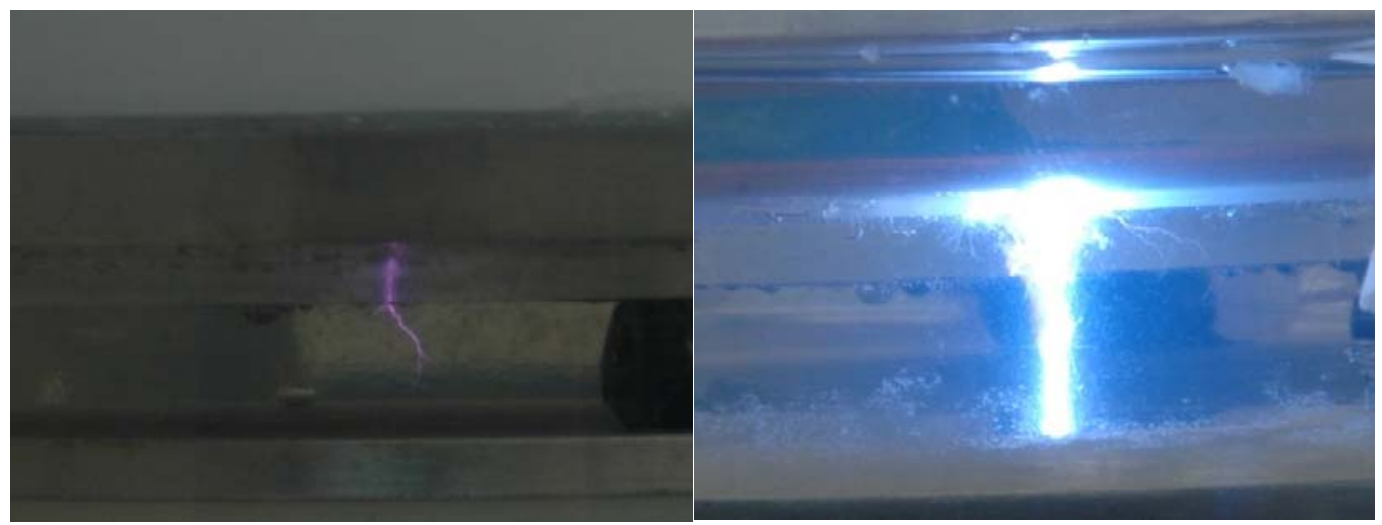

Figure 4-4. Images of plasma discharges through a pinhole: (a) pulsed corona; (b) pulsed arc produced at Drexel Plasma Institute.

Another critical issue that researchers are facing is the scaling-up of the plasma source, to increase the volume of an active plasma discharge region for industrial applications with a large water flow rate. Clearly the point-to-plane electrode geometry would be difficult to scale up for such industrial applications. Also it is difficult to discharge uniformly at multiple pinholes. In order to effectively treat a large volume of water with plasma discharges, different approaches could be used including a wire-cylinder geometry (Figures 4-4e and 4-6), a disk geometry (Figures 4-4f and 4-7) and a concentric cylinder geometry with a HV center composite electrode coated by a thin layer of porous ceramic (Figures $4-4 \mathrm{~g}$ and $4-8$ ). 
Figure 4-6 shows the corona discharge generated using a wire-cylinder type electrode system developed by Malik et al. (2005). A thin tungsten wire $0.075 \mathrm{~mm}$ in diameter was fixed along the axis of a stainless steel tube. A positive high-voltage pulse of up to $90 \mathrm{kV}$ was applied to the wire using a Marx Generator. A pulse duration was $500 \mathrm{~ns}$ full-width half-maximum (FWHM) with an 80-150 ns rise time and a 400-ns fall time was used, so that the streamers emerging from the wire electrode did not reach the outer electrode, and, consequently, could not generate an arc. The energy deposited in the coaxial reactor was $11 \mathrm{~J}$.

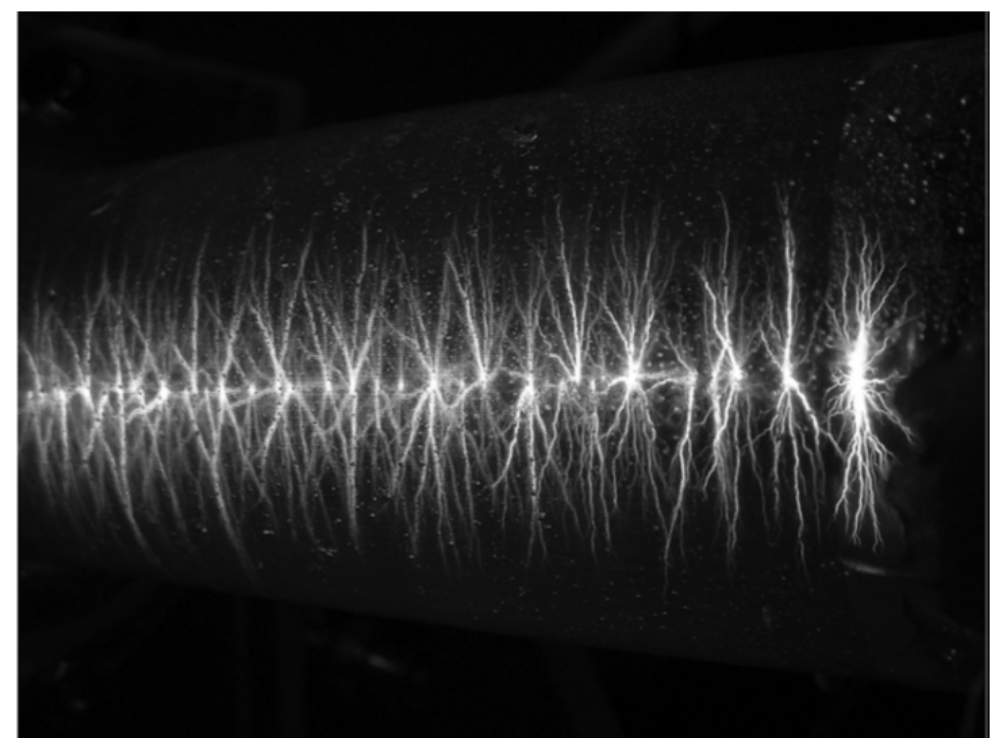

Figure 4-6. Time integrated image of discharges generated using a wire-cylinder geometry in water, where tungsten wire and stainless steel mesh cylinder were used. Chamber dimensions: 44-mm ID, 100-mm length (Malik et al., 2005).

The geometry using multiple disks shown in Fig. 4-5f utilized a number of thin circular stainless steel disk electrodes separated by dielectric layers to produce pulsed multichannel discharges in water. The thickness of the disk electrodes was about $20 \mu \mathrm{m}$. An outer cylindrical stainless steel case was used as the ground electrode, and the gap distance between the inner wall of the cylinder and the edge of the acrylic disks was $5 \mathrm{~mm}$. The electric field at the edge of the stainless steel disk can be estimated as $\mathrm{E} \sim 2 \mathrm{U} / \mathrm{d}$, where $\mathrm{U}$ the applied voltage and $\mathrm{d}$ the disk thickness. Hence high electric field in the order of $10^{6}-10^{7} \mathrm{~V} / \mathrm{cm}$ can be easily achieved, which is comparable to the electric field at the tip of a point electrode. The electric field strength would stay relatively constant throughout the discharge process as long as the thickness of the disk stays constant, thus eliminating the concern of the decay of electric field due to the erosion of a point electrode. The stainless steel disks were sandwiched between pairs of acrylic disks with a diameter of $105 \mathrm{~mm}$ and a thickness of $5 \mathrm{~mm}$. The diameter of the acryl disk was slightly greater than that of the stainless steel disk so that when high voltage was applied on the stainless steel disk, the ionic pre-breakdown current was limited to a small area enclosed by the acrylic 
disks and the edge of the stainless steel disk. Limiting the current to the small area allowed water to be heated and vaporized through joule heating, thus effectively promoting the initiation of the plasma discharges in these vaporized areas. The mechanism is similar to that of the pinhole discharge mentioned previously. However, diaphragm discharge is usually produced through a small hole, leading to very limited treatment capacity. The current design allows the generation of plasma along the periphery of the disk, resulting in a much larger treatment volume. Furthermore, the entire electrode system can be easily scaled up by stacking multiple metal disks for large flow rate water treatment. Figure 4-7 shows photographs of pulsed multichannel discharge arrays generated with one and two stainless steel disks.
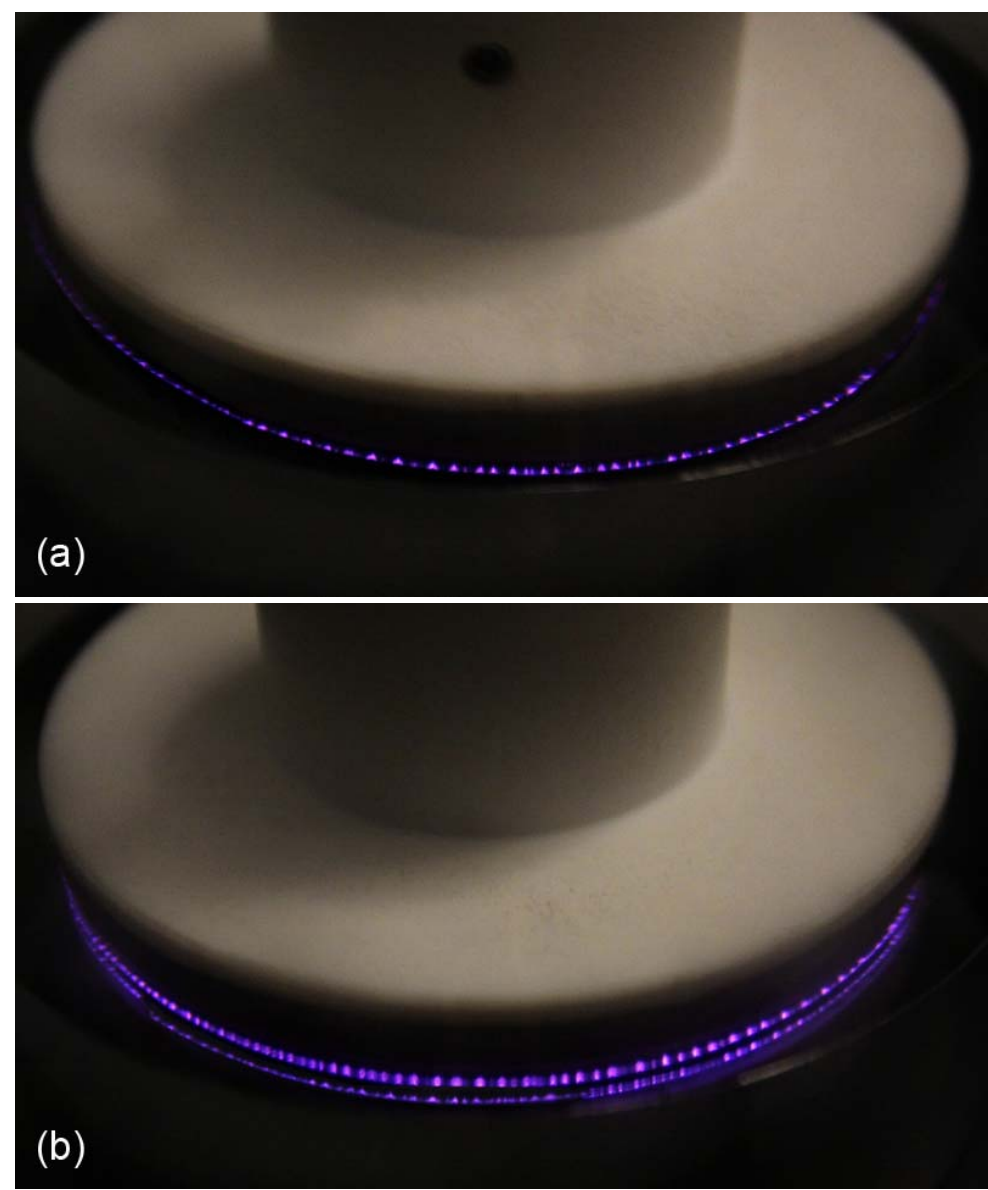

Figure 4-7. Pulsed multichannel discharge array in water generated by two stainless steel disk electrodes separated by a dielectric layer (Yang et al., 2011).

Sunka and his coworkers developed a HV composite electrode coated by a thin layer of porous ceramic to produce large-volume corona discharge in water (Lukes et al., 2008). Such an electrode can be used in a wide variety of geometrical configurations, including wire cylinder and planar geometry. The role of the ceramic layer is to enhance the electric field on the anode 
surface by the concentration of the pre-discharge current in small open pores so that a large number of discharge channels could be distributed uniformly and homogeneously on the electrode surface. The composite electrodes can be made in various dimensions, enabling the construction of reactors that can operate at average power in the range of $\mathrm{kW}$. Figure 4-8 shows images of multichannel pulsed electrical discharges in water generated using porous-ceramiccoated metallic electrodes.

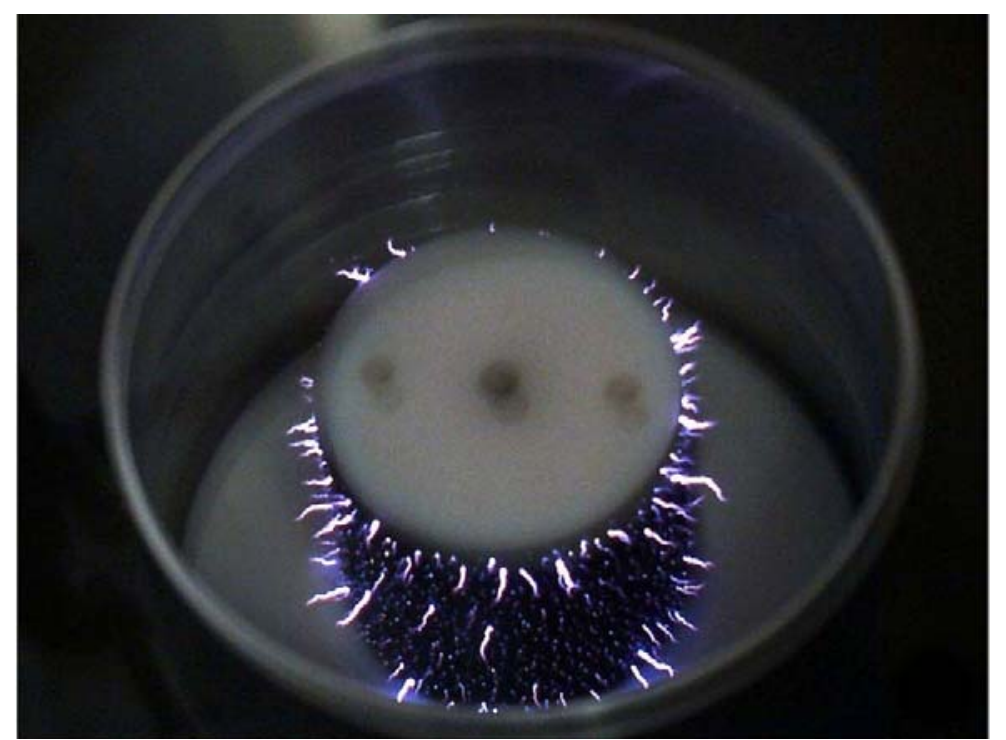

Figure 4-8. Multichannel pulsed electrical discharge in water generated using porous-ceramiccoated metallic electrodes (Lukes et al., 2008).

\subsubsection{Bubble Discharges in Liquid}

In engineering applications of plasma discharges in liquids, high voltage, high-power discharges are often needed for the generation of breakdown in liquids as well as for desired processing. In these cases, the high energy supplied by a power source is first used to evaporate the liquid adjacent to the HV electrode, generating gas bubbles that are subsequently ionized by large electric fields caused by the high voltage. Liquid temperatures in such applications are usually high, at least locally near the breakdown locations, due to the excess power dissipated in the liquid. However, in some circumstances high temperature is not desired. For such applications, a non-thermal plasma system that can generate gas-phase plasmas in contact with liquids is often desired. From practical point of view, these discharges hold another advantage that gas-phase plasmas are much easier to obtain than direct plasma in liquid. Since the gasphase plasma can only interact with the liquid through the gas-liquid interface, a maximization of the interface area is usually desired, which can be achieved by using bubble plasmas, i.e. plasmas generated in small bubbles suspended in liquid. Note that the ratio of the area of gasliquid interface to the total gas volume is inversely proportional to the radius of the gas bubbles. Some of the examples have been shown in Figure 4-9. 
Similar to direct discharges in water, the most commonly used configuration is the point-toplane configuration, where the point electrode was made of a small diameter hollow tube to inject gas into water. Different types of gas were used depending on applications. For example, oxygen gas was often used to promote the formation of oxygen radicals.

Alternatively, gas was bubbled between two metal electrodes (Fig. 4-9b). The discharge occurred between the electrodes by applying the $\mathrm{HV}$ voltage, producing $\mathrm{OH}$ radical that was detected by a spectroscopic technique.

Another interesting discharge in liquid was to use a gas channel, inside which two metal electrodes were placed to generate plasma discharge (Fig. 4-9c). The gas is continuously supplied through the hollow tube, flowing around the electrodes from both sides and exiting from the open ends at the middle of the reactor (see Fig. 4-9c). The gases coming from the top and bottom merge into one where two point electrodes were closely positioned, forming a stable gas channel between the two metal electrodes. Subsequently, the generated discharge was an arc discharge which was cooled and stabilized by the surrounding water.

Aoki and his coworkers studied RF-excited discharges in argon bubbles in a dielectric covered metal rod and wire reactor (Fig. 4-9d). First, bubbles were formed in front of the slot antenna (see black area in the figure) by microwave heating of water where water in an evacuated vessel at a vapor pressure of $5 \mathrm{kPa}$ was evaporated by a slight increase in the temperature above the boiling point (room temperature). In the second step, microwave breakdown took place inside bubbles filled with water vapor. In the third step, the bubbles containing the plasma moved up due to the upward force by buoyancy. After that, new water filled the vacant space in front of the slot antenna. These steps successively repeated forming a large number of bubble plasmas. Microwave-excited plasma in water with or without externally introduced bubbles (Fig. 4-9e) was studied by Ishijama and Nomura (2010). 


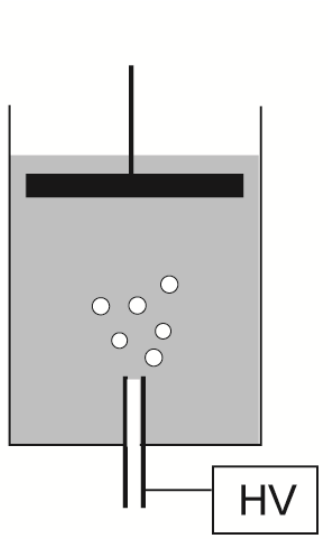

(a)

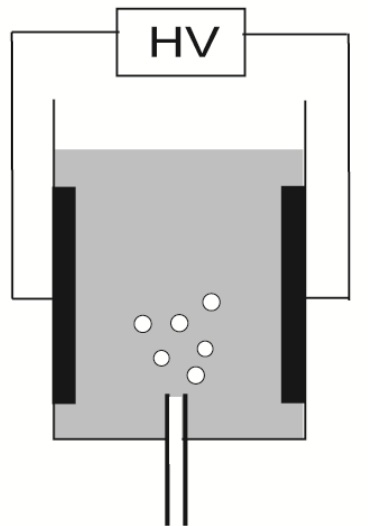

(b)

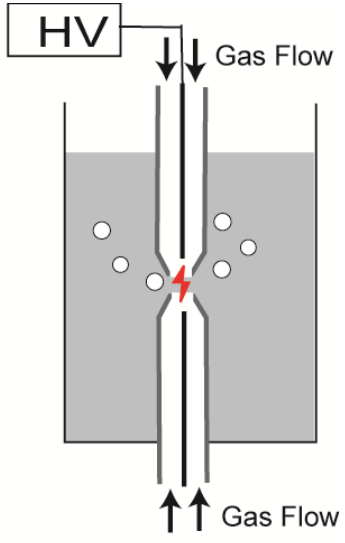

(c)

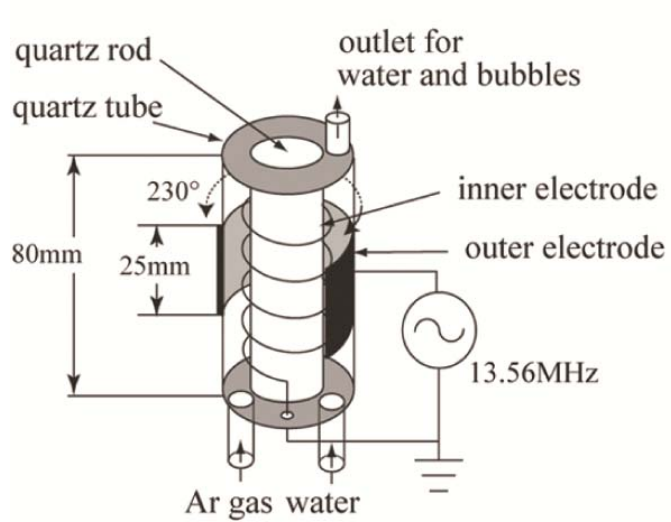

(d)

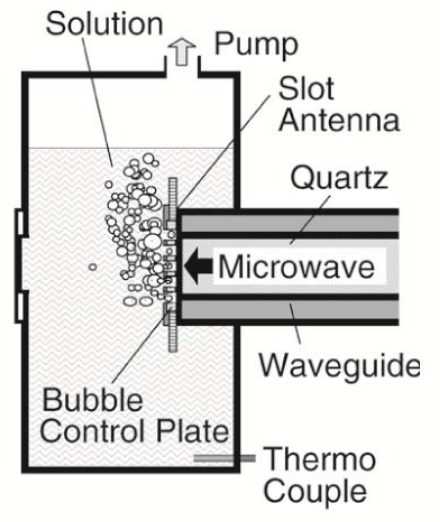

(e)

Figure 4-9. Schematics of electrode geometries for bubble discharge: (a) point-to-plane; (b) parallel plate; (c) gas channel with liquid wall; (d) RF bubble discharge (Hironori, 2008); (e) microwave bubble discharge (Ishijima, 2010). 


\section{Chapter 5 BUBBLE AND ELECTRONIC INITIATION MECHANISM}

Since the very early stage of plasma-in-liquid-related research, two radically different theories for the electric breakdown of liquid were proposed to describe the initiation process. According to the first approach, the electric discharge in liquid initiates from gas cavities either pre-existing in the liquid or on the electrodes, or newly formed through electrolysis, boiling or electron decomposition. This theory is usually referred to as the bubble mechanism. According to the other theory, the discharge is a consequence of avalanche multiplication of free charge carriers in the liquid similar to the gas discharge model generalized to the liquid phase, where electrons in strong fields are accelerated, ionizing molecules and atoms. This discharge theory is conventionally called electronic mechanism.

In this chapter, the two competing theories will be discussed. First, we will start from the well-established classical plasma discharge physics in gas phase to introduce some background knowledge.

\subsection{Electrical Breakdown in Gas Phase}

\subsubsection{The Townsend Breakdown Mechanism}

Electric breakdown in gas phase is a complicated multistage threshold process that occurs when the electric field exceeds some critical value. During the short breakdown period, typically 0.01 to $100 \mu \mathrm{s}$, the gas becomes conductive and, as a result, generates different kinds of plasmas. The breakdown mechanisms can be very sophisticated, but all of them usually start with the electron avalanche, e.g., multiplication of some primary electrons in cascade ionization. Consider first the simplest breakdown in a plane gap with inter-electrode distance $d$, connected to a DC power supply with voltage $\mathrm{V}$. The two electrodes provide a quasi-homogeneous electric field $\mathrm{E}=\mathrm{V} / \mathrm{d}$. It is apparent that an occasional formation of primary electrons near the cathode occurs, providing the very low initial current $i_{0}$. Each primary electron drifts to the anode, concurrently ionizing the gas between the electrodes and generating an avalanche (see Figure 5 -1). The avalanche evolves in time and in space because the multiplication of electrons proceeds along with their drift from the cathode to the anode.

It is convenient to describe the ionization in an avalanche, not by the ionization rate coefficient, but rather by the Townsend ionization coefficient $\alpha$ which can be defined as the electron production per unit length or the number of ionization per unit length. Accordingly, the rate of change of the electron density with respect to $x$ becomes:

$$
\frac{d n_{e}}{d x}=\alpha n_{e}
$$

Thus, the multiplication of electrons (with an initial electron density $\mathrm{n}_{\mathrm{e} 0}$ ) per unit length along the electric field can be given as: 


$$
n_{e}(x)=n_{e 0} \cdot \exp (\alpha x)
$$

The Townsend ionization coefficient is related to the ionization rate coefficient $\mathrm{k}_{\mathrm{i}}\left(\mathrm{E} / \mathrm{n}_{0}\right)$ and electron drift velocity $v_{d}$ as:

$$
\alpha=\frac{v_{i}}{v_{d}}=\frac{k_{i}\left(E / n_{0}\right) n_{0}}{v_{d}}=\frac{1}{\mu_{e}} \frac{k_{i}\left(E / n_{0}\right)}{E / n_{0}}
$$

where $v_{\mathrm{i}}$ is the ionization frequency with respect to single electron and $\mu_{\mathrm{e}}$ is the electron mobility (defined as $v_{d} / E$ ). Noting that breakdown begins at room temperature and that electron mobility is inversely proportional to pressure, it is convenient to present the Townsend coefficient as the similarity parameter $\alpha / \mathrm{p}$ depending on the reduced electric field $\mathrm{E} / \mathrm{p}$.

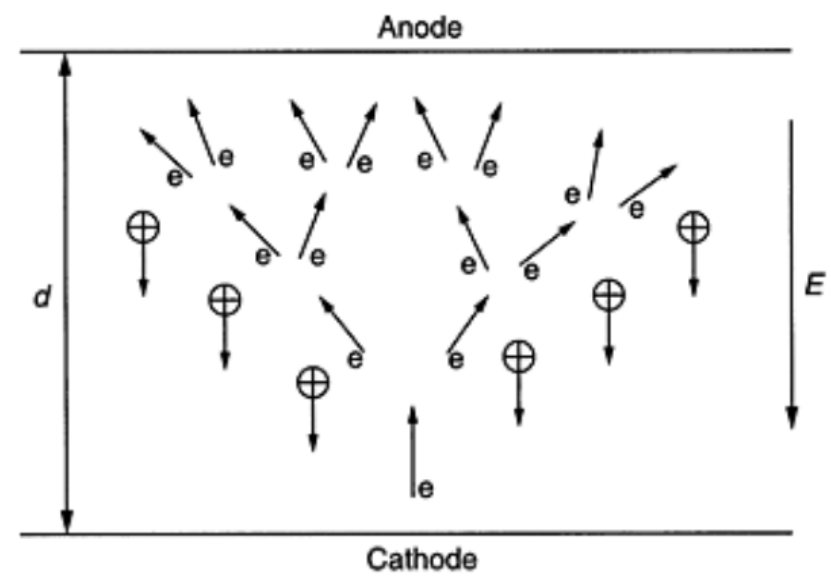

Figure 5 -1. Townsend breakdown gap.

According to the definition of Townsend coefficient $\alpha$, each primary electron generated near the cathode produces $\exp (\alpha \mathrm{d})-1$ positive ions in the gap. (Note that here the electron losses due to recombination and attachment to electron negative molecules were neglected for simplicity.) All the positive ions produced in the gap per one electron are moving toward the cathode and altogether produce $\gamma \cdot[\exp (\alpha \mathrm{d})-1]$ electrons from the cathode, a process which is described as the secondary electron emission. Here, another Townsend coefficient $\gamma$ is the secondary emission coefficient, characterizing the probability of the secondary electron generation on the cathode by an ion impact. The secondary electron emission coefficient $\gamma$ depends on cathode material, the state of surface, the type of gas, and bombarding ion energy (depends on reduced electric field $\mathrm{E} / \mathrm{p}$ ). Typical values of $\gamma$ in discharge range from 0.001 to 0.1 . Taking into account the current of primary electrons $i_{0}$ and electron current due to the secondary electron emission from the cathode, the total electronic part of the cathode current $i_{\text {cath }}$ can be written as: 


$$
i_{\text {cath }}=i_{0}+\gamma i_{\text {cath }}[\exp (\alpha d)-1] \quad(5-4)
$$

The total current in the external circuit is equal to the electronic current at the anode because of the absence of ion current. For this reason the total current can be found as

$$
i=i_{\text {cath }} \exp (\alpha d)
$$

The above two equations lead us to the Townsend Formula, which was first derived by J. S. Townsend in 1902 to describe the ignition of electric discharges:

$$
i=\frac{i_{0} \exp (\alpha d)}{1-\gamma[\exp (\alpha d)-1]} \quad(5-6)
$$

The current in the gap is non-self-sustained as long as the denominator in the above equation is positive. As soon as the electric field, and therefore, the Townsend coefficient $\alpha$, becomes sufficiently large, the denominator in the above equation goes to zero, indicating transition to self-sustained current and thus breakdown takes place. Thus, the simplest breakdown condition in the gap can be expressed as:

$$
\gamma[\exp (\alpha d)-1]=1
$$

or

$$
\alpha d=\ln \left(\frac{1}{\gamma}+1\right)
$$

This process of the ignition of a self-sustained current in a gap controlled by secondary electron emission from the cathode is usually referred to as the Townsend breakdown mechanism.

To derive relations for the breakdown voltage and electric field based on Equation 5 -8, we can rewrite Equation 5 -3 for the Townsend coefficient $\alpha$ in the following conventional semiempirical way:

$$
\frac{\alpha}{p}=A \exp \left(-\frac{B}{E / p}\right)
$$

Equation $5-9$, initially proposed by Townsend, relates the similarity parameters $\alpha / \mathrm{p}$ and $\mathrm{E} / \mathrm{p}$. The parameters A and B for numerical calculations of $\alpha$ in different gases at $E / p=30-500$ $\mathrm{V} / \mathrm{cm} \cdot$ Torr, are given in Table $5-1$. 
Table 5 -1 Numerical parameters A and B for calculation of Townsend coefficient $\alpha$ (Fridman, 2008)

\begin{tabular}{|c|c|c|c|c|c|}
\hline Gas & $\begin{array}{c}\mathrm{A}, \\
1 / \mathrm{cm} \cdot \text { Torr }\end{array}$ & $\begin{array}{c}\mathrm{B}, \\
\mathrm{V} / \mathrm{cm} \cdot \text { Torr }\end{array}$ & Gas & $\begin{array}{c}\mathrm{A}, \\
1 / \mathrm{cm} \cdot \text { Torr }\end{array}$ & $\begin{array}{c}\mathrm{B}, \\
\mathrm{V} / \mathrm{cm} \cdot \text { Torr }\end{array}$ \\
\hline Air & 15 & 365 & $\mathrm{~N}_{2}$ & 10 & 310 \\
\hline $\mathrm{CO}_{2}$ & 20 & 466 & $\mathrm{H}_{2} \mathrm{O}$ & 13 & 290 \\
\hline $\mathrm{H}_{2}$ & 5 & 130 & $\mathrm{He}$ & 3 & 34 \\
\hline $\mathrm{Ne}$ & 4 & 100 & $\mathrm{Ar}$ & 12 & 180 \\
\hline $\mathrm{Kr}$ & 17 & 240 & $\mathrm{Xe}$ & 26 & 350 \\
\hline
\end{tabular}

Combining Equations 5 -8 and 5 -9 gives the following formulas for calculations of breakdown voltage and breakdown reduced electric field as functions of the important similarity parameter pd:

$$
\begin{aligned}
& V=\frac{B(p d)}{C+\ln (p d)} \\
& \frac{E}{p}=\frac{B}{C+\ln (p d)}
\end{aligned}
$$

In these formulas, the parameter $\mathrm{A}$ is replaced by $\mathrm{C}=\ln \mathrm{A}-\ln \ln (1 / \gamma+1)$, taking into account the weak, double logarithmic influence of the secondary electron emission.

The breakdown voltage dependence on the parameter pd, as described by Equation 5 -10 and $5-11$ is usually referred to as the Paschen curve. The experimental Paschen curves for different gases are presented in Figure 5 -2. 


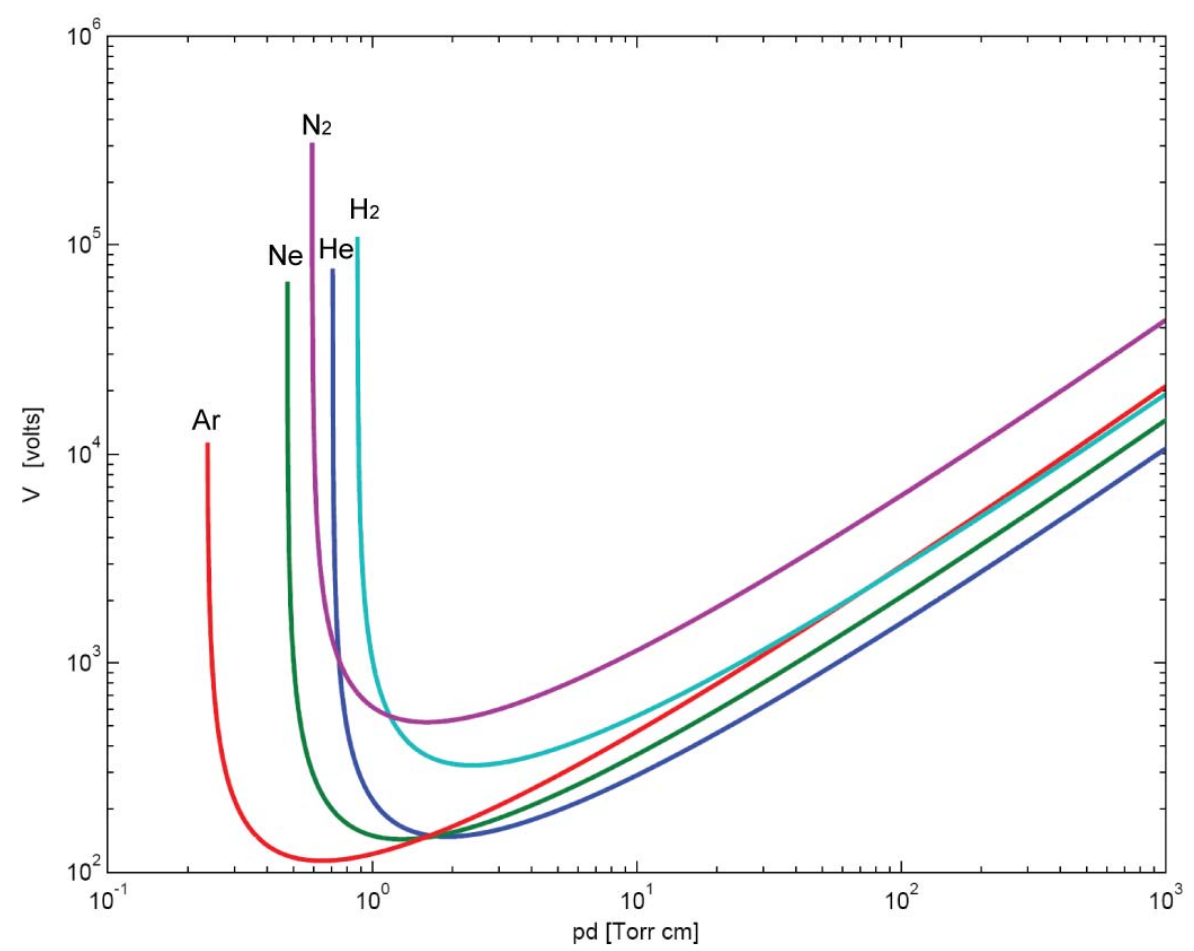

Figure 5 -2. Paschen curves for different gases. (Fridman, 2008)

The curves in Figure $5-2$ have minimum voltage points corresponding to the easiest breakdown conditions that can be found from Equation 5 -11:

$$
\begin{aligned}
& V_{\text {min }}=\frac{e B}{A} \ln \left(1+\frac{1}{\gamma}\right) \\
& \left(\frac{E}{p}\right)_{\text {min }}=B \\
& (p d)_{\text {min }}=\frac{e}{A} \ln \left(1+\frac{1}{\gamma}\right)
\end{aligned}
$$

where $\mathrm{e}=2.72$ is the base of natural logarithm.

\subsubsection{Spark Breakdown Mechanism}

The Townsend mechanism described in the previous section can only be applied for quasihomogeneous breakdowns for low pressures and short discharge gaps. Another breakdown mechanism, the so-called spark breakdown mechanism, or streamer breakdown mechanism, takes place in larger gaps at high pressures. In contrast to the Townsend mechanism, the spark mechanism provides breakdown in a local narrow channel with very high current and current density, but without direct relation to the electrodes and secondary electron emissions. 
Both spark and Townsend breakdowns are primarily related to avalanches. However, in large gaps breakdowns cannot be considered independent and stimulated by electron emission from cathode. The spark breakdown at higher $p d$ and considerable overvoltage develops much faster than the time necessary for ions to cross the gap and provide the secondary electron emission. The high conductivity spark channel can be formed even faster than electron drift time from cathode to anode. In this case, the breakdown voltage is independent of the cathode material.

The mechanism of spark breakdown is based on the concept of streamer. The spark breakdown occurs at a thin ionized channel which grows fast along the positively charged trail left by an intensive primary avalanche between two electrodes. The avalanche also generates photons, which in turn initiate numerous secondary avalanches in the vicinity of the primary one. Electrons of the secondary avalanches are pulled by the strong electric field into the positively charged trail of the primary avalanche, creating a streamer propagating fast between the two electrodes. The streamer theory was originally developed by Raether (1964), Loeb (1960), and Meek and Craggs (1978).

As mentioned in the previous section, each primary electron generated near the cathode produces $\exp (\alpha x)-1(\approx \exp (\alpha x)$ when distance is large $)$ positive ions in the gap. In this case, $\exp (\alpha x)$ can be regarded as the ratio of charge amplification, and considerable space charge can be created with its own significant electric field $E_{a}$, which should be added to the external field $\mathrm{E}_{0}$. The electrons are at the head of the avalanche while the positive ions remain behind, creating a dipole.

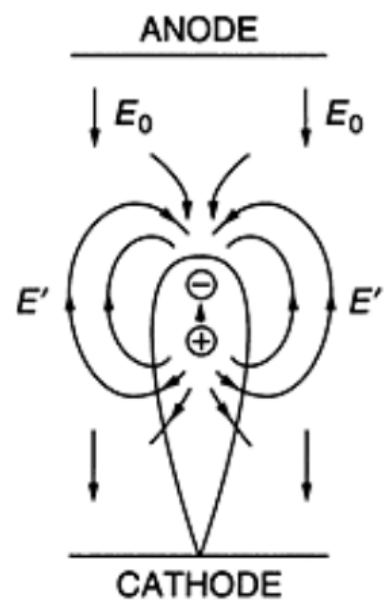

(a)

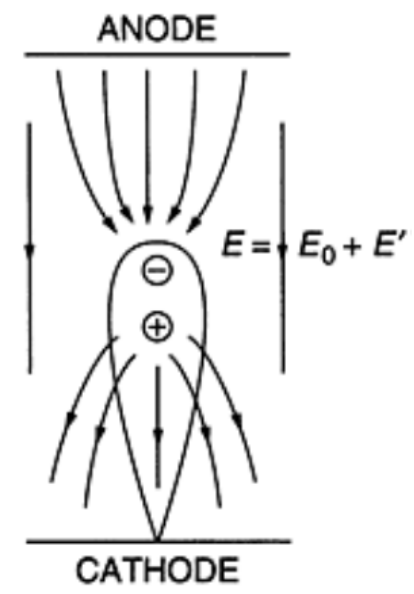

(b)

Figure 5 -3. Electric field distribution in an avalanche. 
The external electric field distortion due to the space charge of the dipole is depicted in Figure 5 -3. In front of the avalanche head, the external $E_{0}$ and internal E' electric fields make a total field stronger, which in turn accelerates ionization. On the contrary, in between the separated charges, or "inside the avalanche", the total electric field is reduced, which slows down the ionization. At some critical value of $\alpha \mathrm{x}$, the electric field induced by charge $\mathrm{N}_{\mathrm{e}} \approx$ $\exp (\alpha x)$ reaches the value of the external field $\mathrm{E}_{0}$. Then the avalanche to streamer transformation takes place when the internal field of an avalanche becomes comparable with the external electric field, e.g., when the amplification parameter $\alpha \mathrm{x}$ is sufficiently large.

As soon as the avalanche head reaches the anode, the electrons sink into the anode and it is mostly the ionic trail that remains in the discharge gap. The electric field distortion due to the space charge in this case is shown in Figure $5-4$. Because electrons are no longer present in the gap, the total electric field is due to the external field, the ionic trail. The field can also be viewed as a result of the ionic charge image in the anode. The resulting electric field in the ionic trail near the anode is less than the external electric field. But at spots farther away from the electrode, it exceeds $\mathrm{E}_{0}$, inducing the formation of so-called cathode directed streamer.

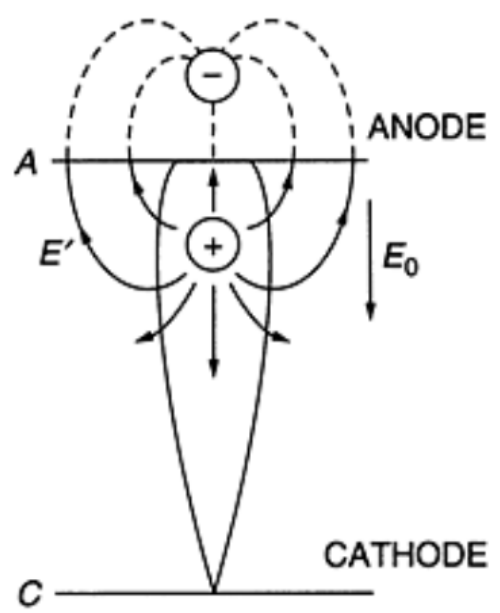

(a)

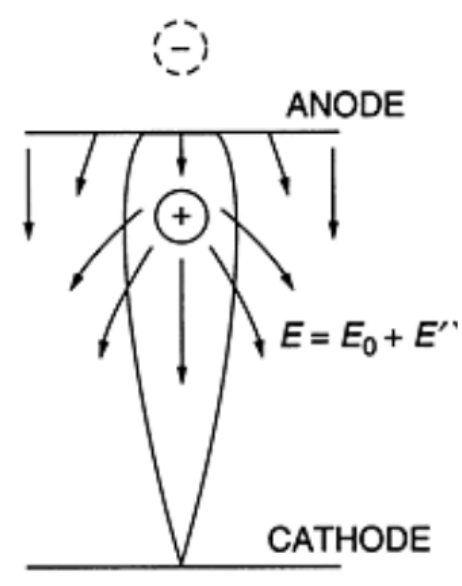

(b)

Figure 5 -4. Electric field distribution when the avalanche reaches the anode.

Energy photons emitted from the primary avalanche provide photoionization in its vicinity, which initiates secondary avalanches. Electrons of the secondary avalanches are pulled into the ionic trail of the primary one due to the enhanced electric field (i.e., $\mathrm{E}_{0}+\mathrm{E}^{\prime}$ ) as shown in Figure 5 -4 . The process repeats, providing the growth of the streamer like a "needle" from the anode. Obviously, the electric field at the tip of the "anode needle" is very large, which stimulates the fast streamer propagation in the direction of the cathode. 


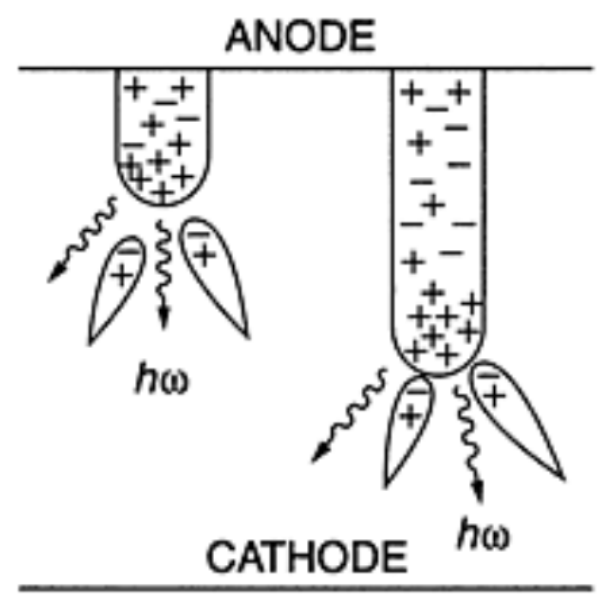

(a)

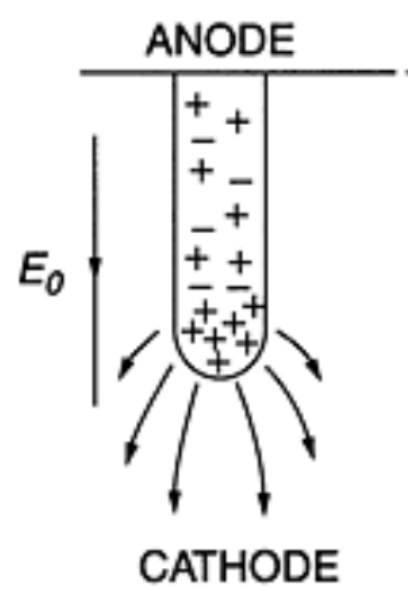

(b)

Figure 5 -5. Cathode directed streamer.

Note that streamers are not always cathode-directed. If the overvoltage is high or the electrode distance is large, the avalanche-to-streamer transformation can take place before the avalanche head reaches the anode. In this case, the so-called anode-directed streamer is able to grow toward both electrodes.

In 1940, Meek proposed his criterion for streamer formation on a quantitative basis, which can be presented as the requirement for the avalanche amplification parameter $\alpha \mathrm{d}$ to exceed a critical value of 20. This convenient and important criterion is known as the Meek's criterion.

\subsection{Electron Avalanche for Electrical Breakdown in Liquid Phase}

Comparing to electrical breakdown in gas, there is no adequate model of discharge development in liquid phase. While electron avalanche ionization and streamer mechanisms were generally accepted to explain the breakdown in gases, the nature of the breakdown in dielectric liquids is still disputed. The main problem is a completely different interaction dynamics in liquid systems compared to the gas-phase systems. In liquids, multi-particle interactions cannot be neglected. These interactions lead to two basic phenomena: 1) electronmolecule interaction cross-sections measured in the gas phase cannot be used in liquids; for example, cross-section shapes and even thresholds change because of molecule-to-molecule interactions; 2) Boltzmann equation for electron energy distribution function (EEDF) has to take into account multi-particle collisions; in other words, collision integral cannot be calculated using a binary collision approximation in this case.

Thus we have to underline that now we have no theoretical approaches to model the discharge development in condensed liquid media. Nevertheless there are two models usually used for qualitative analysis: "dense gas" approximation and "semiconductor" approximation. "Dense gas" approach assumes that the liquid is a gas with high particle number density. This approach neglects both fundamental problems of the process description in condensed media 
and could lead to unpredictable errors. However, from the other point of view the model can provide at least qualitative estimations for the process, so that we will be able to use this approach to estimate the discharge parameters. "Semiconductor" approach approximates liquid as a solid-state crystal with semiconductor properties. The current through the liquid media could be described on the basis of tunneling of electron-hole pairs in structured potential. This model also has obvious drawbacks but in some cases can provide a reasonable estimation of conductive current through dielectric liquids. We will briefly review both approximations.

\subsubsection{Dense Gas Approximation}

The dominant collisions in gas phase usually involve two particles, although under some circumstances three-body interactions can be important. In liquid, the different electronmolecule interaction behavior rises mostly because of multi-body collisions due to not only the much higher liquid density but also the stronger chemical bonds between neutral particles. To make the case simpler, the dense gas approximation assumes that two body collisions are still applicable: there is no fundamental difference between liquid and gas, and the only different parameter is the density of neutral particles.

Understanding the complex interactions involved when atoms or molecules are ionized by electron impact remains, at the present time, one of the greater challenges of atomic physics. The data on the interaction between electron and liquid molecules comes mainly from e-beam or radiolysis experiments, and cross sections for the high-density liquids became known only very recently (see Figure $5-6$ ). From the collisional data, one can obtain the fluid transport parameters such as electron mobility and diffusion coefficient. Monte Carlo simulations have been performed by several groups that give the electron elastic collision, ionization and recombination rates. 


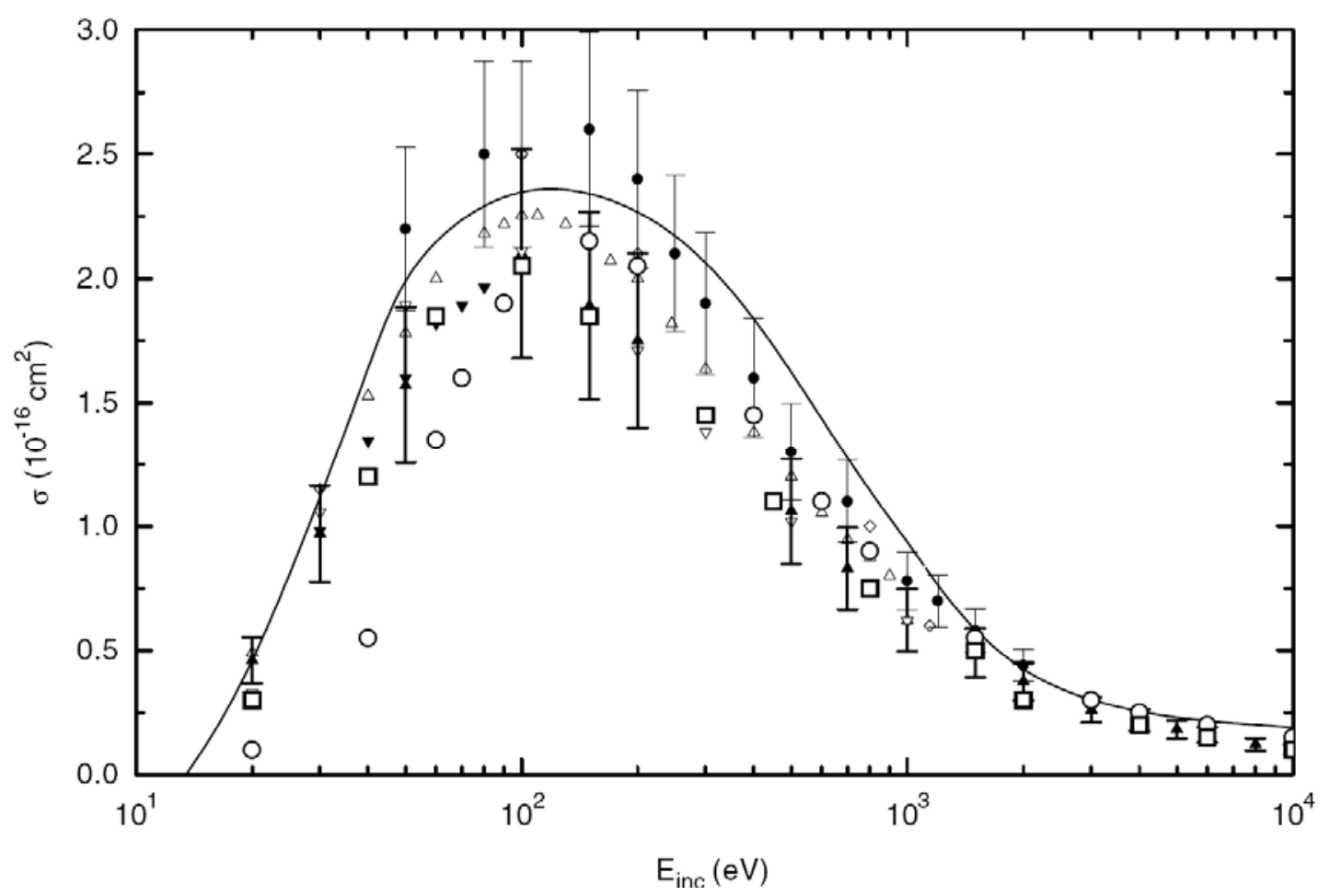

Figure 5 -6. Comparison between the calculated ionization cross section of the vapour-water molecule by Champion (2003) (solid line) and experimental data: Bolorizadeh and Rudd (1985) (solid circles), Djuric et al (1988) (solid down triangles), Schutten et al (1966) (solid up triangles), Khare and Meath (1987) (open down triangles), Straub et al (1998) (open up triangles) and Olivero et al (1972) (open diamonds). By comparison, the results obtained by Dingfelder et al (1998) (open circles)

\subsubsection{Semiconductor Approximation}

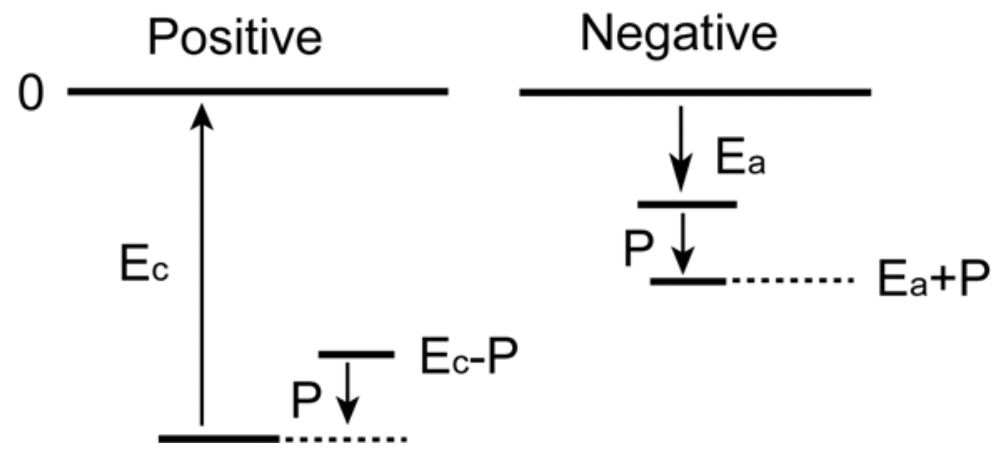

Figure 5-7 Electronic energy states for positive and negative molecular ions in gaseous phases 
In considerations of charge transfer at the electrode interface, emphasis has traditionally been placed on field-controlled electron emissions. However, this approach is significantly compromised by due to the fast hydration of free electrons. In dielectric liquids, charged particles are normally present through the dissociative ionization of a neutral molecule:

$$
M \leftrightarrow A^{+}+B^{-}
$$

The degree of the dissociative ionization depends highly on the short and long range binding forces. Usually dissociations are more readily to occur in polar liquids, water for instance, than non-polar liquids because the high permittivity will weaken the Coulomb attraction between the anion and cation pairs. In liquid water, the proton $\mathrm{H}^{+}$combines with a $\mathrm{H}_{2} \mathrm{O}$ molecule to form the $\mathrm{H}_{3} \mathrm{O}^{+}$complex. This complex can transfer an excessive proton to the neighboring water molecule. This molecule turns in to $\mathrm{H}_{3} \mathrm{O}^{+}$complex, and the transferring process can be passed on via the displacement of electrons, while the proton can be regarded as stationary. The charge carriers move under the strong electric field by the mechanism described above, obtaining enough energy and eventually causing the ionization. This hopping process of the charge particles is very similar to the hole mechanism in semiconductor physics, and hence called the semiconductor approximation (Lewis, 1994).

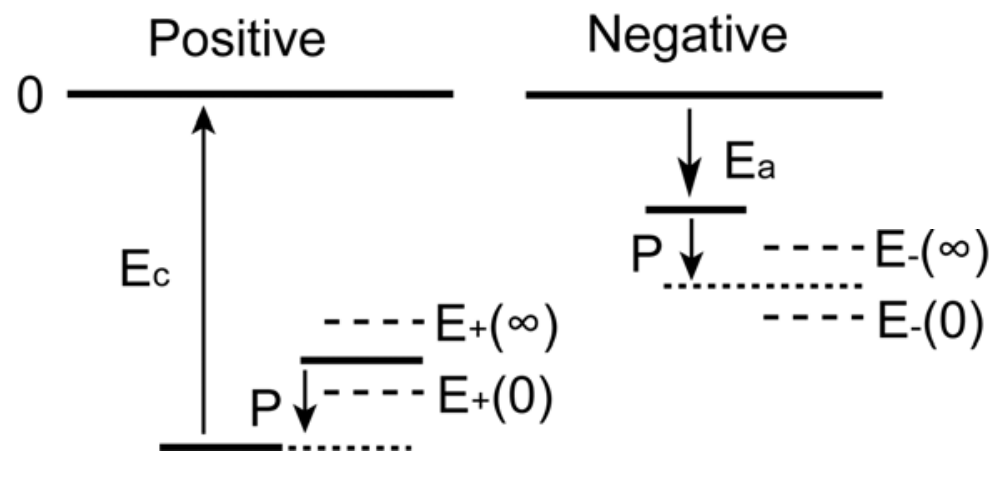

Figure 5 -8 The localized $\mathrm{E}(\infty)$ and de-localized $\mathrm{E}(0)$ energy states of positive and negative ions in a liquid.

For gaseous state, the positive and negative ions will have energy level of $E_{c}$ and $E_{a}$, respectively, due to the loss/gain of the electrons. The cases in liquid are more complicated, mostly due to the solvation of the ions. The respective energy levels have to been modified by the collective polarization responses of the molecules surrounding the ions. The local reorganization of liquid molecules consists of two parts: the electronic component which will grow on a time scale comparable with that needed to relocate the electron, and the atomic component which can be considered frozen at initial stage considering the Frank-Condon 
principle and will take a much longer time to form. The former causes the positive and negative ion energies $\left(E_{c}\right.$ and $\left.E_{a}\right)$ in the liquid to become $E_{c}-P$ and $E_{a}+P$, respectively, where $P$ is the associated electronic solvation energy (as shown in Figure 5 -7). The typical value for $\mathrm{P}$ is about $1 \mathrm{eV}$ for non-polar liquids with relative permittivity of 2-3, and several eVs for liquids with high dielectric permittivity (such as water, with $\varepsilon_{\mathrm{r}}=80$ ).

Considering the relative slowness of the atomic reorganization, the energy level will be further shifted by an energy amount of $\lambda$. Due to the different time required for $\mathrm{P}$ and $\lambda$ shifts, it is possible to define two electronic states for each ion. In the case of the negative ion state the energy is E. $(\mathrm{O})$ at the moment an electron is localized at a molecular site and E_ $(\infty)$ when the electron is in a fully polarized state after reorganization has occurred. Likewise, for a positive ion (hole) state, $\mathrm{E}_{+}(\mathrm{O})$ is the energy of the hole or positive ion at the moment an electron leaves a neutral molecular site while $\mathrm{E}_{+}(\infty)$ is the subsequent energy of the fully polarized positive ion state. The states between $\mathrm{E}(0)$ and $\mathrm{E}(\infty)$ corresponds to various degrees of localization. Note that the edges of $\mathrm{E}(0)$ and $\mathrm{E}(\infty)$ are usually "blurred" by the thermal disturbance of the particles. The band-gap energy [ $\left.\mathrm{E}_{-}(\mathrm{O})-\mathrm{E}_{+}(\mathrm{O})\right]$ between the two sets of states is the necessary energy to create a quasi-free electron-hole pair, as an analogy to the notions from semiconductor physics.

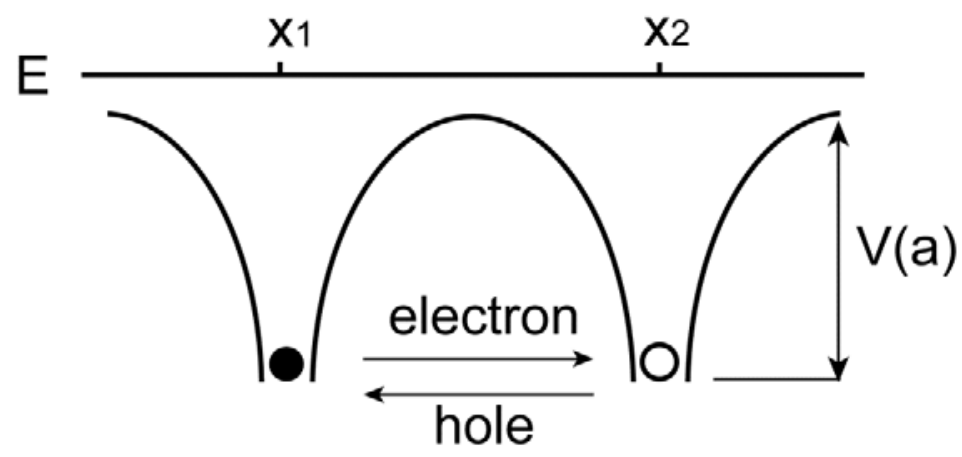

Figure 5 -9 Transfer of electron by tunneling between molecules.

Under some circumstances, high electric field for instance, the electrons and holes which have become localized in dielectric liquids can tunnel from one molecule to the next as shown in Figure 5 -9. If tunneling occurs at an energy $E$ in the band of states through an intermolecular potential barrier V(a), assumed for simplicity to be one-dimensional (Figure 5 -9), then the probability of a transition depends on a factor

$$
f=\exp \left[\frac{-4 \pi}{h} \int_{x_{1}}^{x_{2}}[2 m(V(x)-E)]^{\frac{1}{2}} d x\right.
$$


where $\mathrm{x}_{1}, \mathrm{x}_{2}$ are the spatial limits of $\mathrm{x}$ where $\mathrm{V}(\mathrm{x})=\mathrm{E}$. The probability, hence the rate, of the transition depends strongly on the height of the potential well and the distance between two molecules.

\section{3 "Bubble Theory" for Electric Breakdown in liquid}

Although different electron avalanche mechanisms have been proposed to explain the electrical breakdown in liquid, the nature of the breakdown is still heavily disputed. In gas, the critical breakdown condition is described by the Paschen curve (section 3.1), with which one can estimate the breakdown voltage of atmospheric pressure air, for example. Due to the density difference between air and water, one could expect from the Paschen curve that the breakdown voltage in water would be approximately three orders of magnitude higher than that in air. However, a large number of experiment data on the water breakdown show that this breakdown voltage is higher, but still of the same order of magnitude as for gases. This interesting and important finding can be explained by taking into account the fast formation of gas channels in water under the influence of an applied high voltage. When formed, the gas channel can serve as a medium for electron avalanche, a phenomenon which explains the relative low voltage requirement for water breakdown. The formation of the gas channel can be attributed to through interface processes, fast local heating, evaporation, or pre-existing gas bubbles.

\subsubsection{Bubble Formation: Interface Processes}

The influence of electrical charges on surface is very important to their physical chemistry. To begin with, consider a solid metal surface bearing a uniform positive charge density in contact with a solution phase that contains both positive and negative ions. When the applied electric field is zero, charged layers will be established at the metal-liquid interface mainly due to the contact potential between the metal and the liquid. The metal side of the interface will consist of an electron cloud extending out beyond the positive cores of the metal surface. Under the effect of Coulombic force, the ions in the liquid will be attracted and adsorb chemically or physically on to this layer to form Stern and inner Helmholtz layer as shown in Figure 5 -10. Further outside are the outer Helmholtz layer and diffuse layer, where the net charge decays to zero in the bulk liquid and effectively screen the Stern and inner Helmholtz layer from the bulk of the solution. The structure of the electrical double layer is presented in Figure 5-10. 


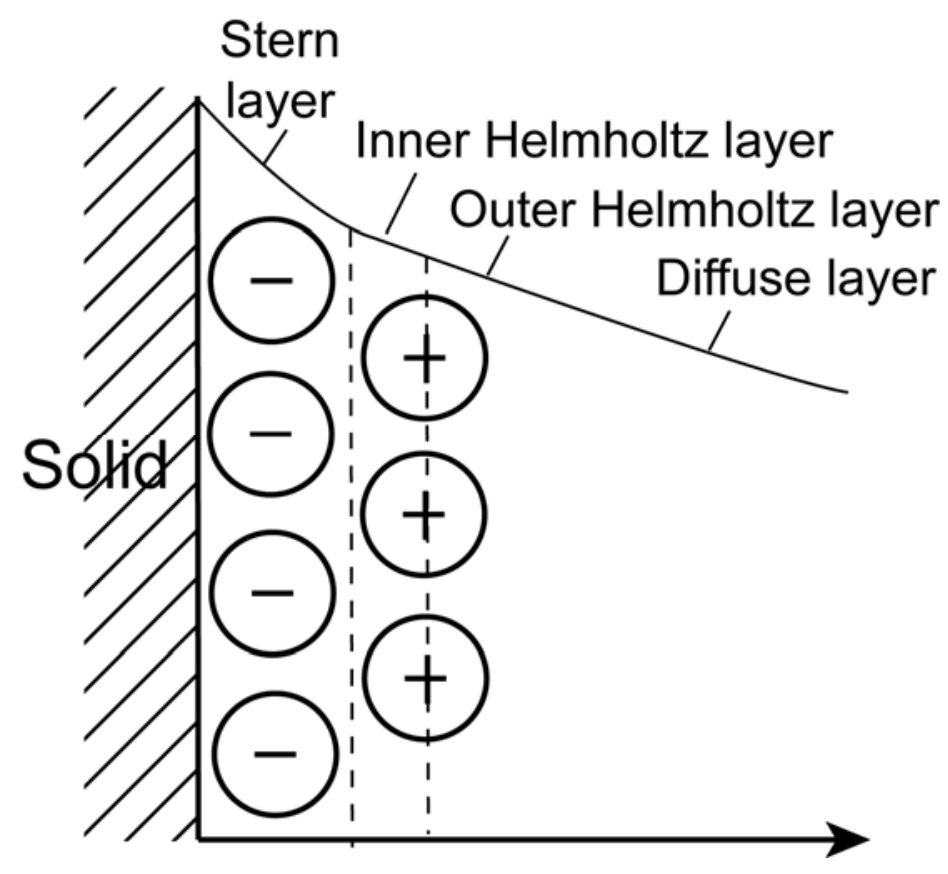

Figure 5 -10. Schematic diagram of electrical double layer on the solid-liquid interface.

The formation of the so-called "bubble" or "void" is usually associated with surface tension $\gamma$ at the interface. Under constant temperature and pressure conditions, the relationship between $\gamma$ and the potential difference $\mathrm{V}$ across the interface can be described by the Lippmann capillary equation:

$$
\frac{d \gamma}{d V}=-q
$$

where $\mathrm{q}$ is the surface charge density on the metal side of the interface. Analysis by Lewis (2003) showed that an applied electric field could alter the electrode interfacial double layer field, and thus change the cohesion of the liquid at the electrode boundary. Under electric stress as high as a breakdown threshold, the cohesion of the liquid can be much reduced or even lost altogether at the electrode surface, and leading to the formation of the "void".

Another factor that may contribute to the breakdown process is the enhanced local field of the electrode double layer that induces the Lippmann process will simultaneously control charge transfer across the electrode-liquid interface. The Auger process can provide both energetic electron injection at a cathode and efficient positive hole or ion inject at an anode. 


\subsubsection{Bubble Formation: Joule Heating}

Another possible mechanism for producing bubbles at the initial stage of electrical breakdown is through Joule heating by ionic conduction inside the liquid. The power of the Joule heating per unit volume $\left(\mathrm{W} / \mathrm{m}^{3}\right)$ is defined as:

$$
P=\sigma E^{2} \quad(5-18)
$$

where $\sigma$ is the electric conductivity of the liquid, and $\mathrm{E}$ is the electric field. Usually, the energy from Joule heating is well below the amount of energy required to heat the entire volume of liquid provided. However, considering that under normal breakdown conditions, an electric field strength over $1 \mathrm{MV} / \mathrm{cm}$ is usually required, significant energy will be deposited through Joule heating at the local region around the tip of an electrode, subsequently creating microbubbles near the electrode tip. If we take distilled water for example, assume the electric conductivity is in the range of a few $\mu \mathrm{S} / \mathrm{cm}$, then the time required for the generation of a gas bubble can be calculated to be a few hundred microseconds using the following properties of water: thermal capacity $C_{\mathrm{p}}=4.186 \mathrm{~kJ} / \mathrm{kg} / \mathrm{K}$, latent heat $\Delta H_{1-\mathrm{g}}=2260 \mathrm{~kJ} / \mathrm{kg}, \mu E^{2} \cdot \Delta t=\rho\left(C_{\mathrm{p}} \Delta T+\right.$ $\Delta H_{\mathrm{l}-\mathrm{g}}$ ) with $\Delta T=75 \mathrm{~K}$. This rough estimation shows that internal heating can only be important if the duration of the applied voltage is sufficiently long. In recent experiments, the breakdown of water was observed with short sub-nanosecond voltage pulses. The time durations in these experiments were certainly several orders of magnitude shorter than that for any significant heating. Details about these nanosecond and sub-nanosecond breakdowns will be discussed in later sections.

\subsubsection{Bubble Formation: Pre-existing Bubbles}

The initiation of breakdown in water with the aid of pre-existing bubbles of the size of 40 $100 \mu \mathrm{m}$ using pulse heated wire electrode was investigated by Korobeynikov et al. (2002). It was observed that the bubbles elongated in the direction of electric field, possibly due to the charge deposition on the gas-liquid interface. With a sufficiently high voltage, the discharge could be initiated in a bubble, with the prebreakdown time in the presence of a bubble being much shorter than in its absence. Another interesting phenomenon is that the bubbles behave differently for negative and positive discharges. When a cathode bubble separates from the electrode surface, it does not participate in the streamer formation, indicating that direct cathodic electron injection in the bubble is of importance in this case.

Monte Carlo simulation was performed to show that a random distribution of pre-existing micro-bubbles within the liquid would adequately explain the observed breakdown fields and time delays (Qian et al., 2006). Polarity differences in hold-off voltages and breakdown initiation times are also in agreement with the micro-bubble models. 


\subsection{Streamer Propagation}

Despite different mechanisms proposed, all the initiation theories lead to the formation of a low density region so that self-sustained electron avalanche could be possible. Thus, the next question is that what the driving force is to sustain and expand the cavity to form complex geometrical structures. Similar to the initiation process, the propagation is complicated because it involves interactions between plasma, gas and liquid phases of the media. Recent experiments demonstrated the existence of different modes of propagation, where both a primary streamer mode with a slow velocity and a secondary streamer mode with a high velocity were observed. Several models have been proposed to correlate electric field to streamer velocity. Different effects, including liquid viscosity, trapping of positive and negative carriers in the conducting channel, and local electric charge at streamer head were taken into account. But again, there is not yet a commonly accepted model.

In the following section, we describe a theoretical framework for understanding the propagation of streamers of electric discharge in water subjected to high voltage. The breakdown process is usually characterized by two typical features of breakdown: rapid propagation of discharge streamers and high tendency of branching and formation of random dendritic structures. Therefore, the present theoretical review consists of two components: quantitative model for possible mechanisms to produce the driving force needed to sustain and promote the propagation, and stability analysis of a single cylindrical filament with surface charges in an external electric field.

\subsubsection{Electrostatic Model}

A schematic diagram of the present electrostatic model is shown in Figure 5-11(a). A thin needle electrode with a rounded tip was aligned perpendicular to a ground plate electrode. High voltage $\Phi_{0}$ was applied on the needle electrode. According to Kupershtokh and Medvedev (2006), liquids could become phase unstable under a high electric field strength so that gas channels could form along electric field lines.

(a)

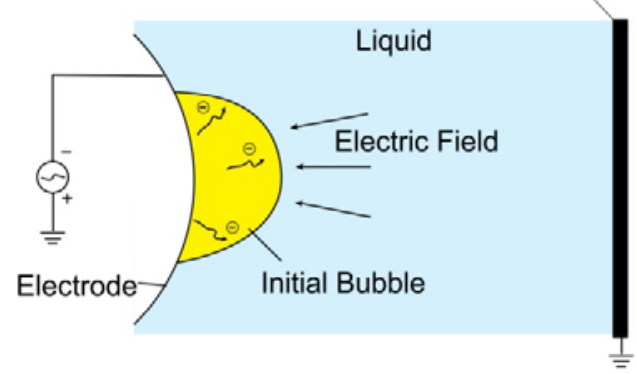

(b) Ground

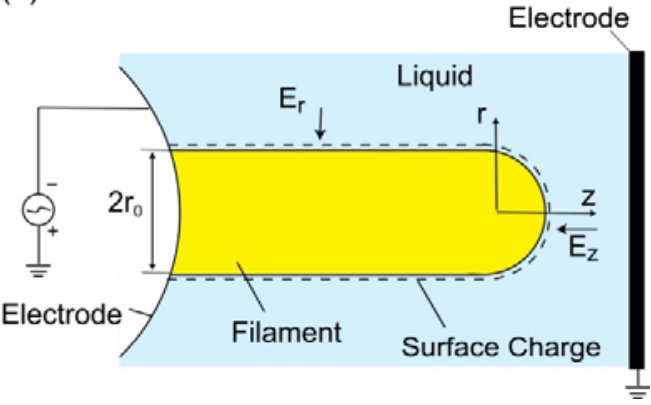

Figure 5 -11 (a) Initiation of bubble formation; (b) schematic diagram of a cylindrical filament in water 
The time required for breakdown ignition in the channels can be estimated as $\tau_{\mathrm{b}}=\left(k_{\mathrm{I}} n_{0}\right)^{-1}$, where $k_{\mathrm{I}}$ is the direct ionization rate coefficient, and $n_{0}$ is the molecule density. Under atmospheric pressure $n_{0}$ is in the order of $10^{19} \mathrm{~cm}^{-3}$, while $k_{\mathrm{I}}$ is in the order of $10^{-10}$ to $10^{-9} \mathrm{~cm}^{3} / \mathrm{s}$ in the reduced electric field $E / n_{0}$ of $10^{3} \mathrm{~V} \cdot \mathrm{cm}^{2}$. Hence, $\tau_{\mathrm{b}}$ is in the order of 0.1 to $1 \mathrm{~ns}$. For negative discharges, due to the higher momentum transfer collision frequency and thus a low mobility in the liquid phase, electrons tend to deposit on the gas-liquid interface and charge it negatively. For positive discharges, the high mobility of electrons would leave the interface charged positively. Under both circumstances, it is possible that the charged interface would be pushed to displace the liquid under external electric field by electrostatic force.

A simplified calculation can be made to examine whether or not the electrostatic force would be sufficient to overcome the resistance of water at the interface. The pressure due to the surface tension, $\gamma$, on a water interface of a spherical bubble with a radius of curvature $r$, can be approximated by the Young-Laplace equation $\mathrm{p}=2 \gamma / \mathrm{r}$. With $\mathrm{r} \sim 1 \mu \mathrm{m}$, and $\gamma=72.8 \times 10^{-4} \mathrm{~N} / \mathrm{m}$ the surface tension pressure is $\sim 15 \mathrm{kPa}$. The ultimate strength of water of approximately 30 MPa must be exceeded for rupturing the liquid. Considering forces due to charged particles only and ignoring those due to field gradients and material property gradients, the electric force at the interface becomes simply the electrostatic force, $\mathrm{L}$, which is the product of charge density per unit area $\sigma$ and the electric field $\mathrm{E}$, i. e., $\mathrm{L}=\mathrm{e} \sigma \mathrm{E}$, where $\mathrm{e}$ is the charge per electron. For $\mathrm{E}=10^{8}$ $\mathrm{V} / \mathrm{cm}, \sigma$ should have a value of $10^{12}$ charges $/ \mathrm{cm}^{2}$. For electrons with an average energy of $1 \mathrm{eV}$, the electron thermal velocity can be estimated as $6 \times 10^{7} \mathrm{~cm} / \mathrm{s}$. So a modest electron density of $10^{13} \mathrm{~cm}^{-3}$ will provide the flux necessary to charge the surface to the breaking point within $1 \mathrm{~ns}$. Although these estimations for water rupturing also neglect both loss mechanisms and the energy requirements to overcome the hydrodynamic resistance, the electrostatic mechanism still seems a likely candidate for streamer propagation, and such forces may dominate at nanosecond time scale.

The growth of a plasma filament is determined by conservation equations of mass, momentum and energy. To quantify the breakdown process described above, the equations for the formation and propagation of the plasma-filled filaments are defined as (Gidalevich and Boxman, 2006):

$$
\begin{aligned}
& \frac{\partial \rho}{\partial t}+\nabla \cdot(\rho u)=\frac{2 \lambda(T) T}{\Delta_{v} H r_{0}{ }^{2}} \\
& \frac{\partial u}{\partial t}+u \cdot \nabla u+\frac{1}{\rho} \nabla P=0 \\
& \frac{\partial}{\partial t}\left(\rho\left(Z+u^{2}\right)\right)+\nabla \cdot\left(\rho u\left(Z+\frac{P}{\rho}+\frac{u^{2}}{2}\right)\right)=\kappa(T) E^{2}
\end{aligned}
$$


where $t$ is time, $\rho$ and $P$ are the radial density and pressure inside streamer, respectively, $u$ is the velocity of streamer, $T$ is the temperature, $\lambda$ is the thermal conductivity, $\Delta_{v} H$ is the evaporation heat of water, $r_{0}$ is the radius of streamer, $Z$ is the internal energy of ionized gas, $E$ is the electric field strength, and $\sigma$ is the electric conductivity. It is usually difficult to directly solve Equations $(5-19)$ - $(5-21)$ because of the high non-linearity of the equations.

For simplification, the streamer was assumed to be a cylinder with a hemispherical tip as shown in Figure 5 -11(b). The reference frame was fixed on the tip. The radius of the filament is $\mathrm{r}_{0}$. Although it appears from photographic evidences that the filament is usually of a conical shape, the cylindrical approach is still a good approximation when the length of the filament is much greater than the radius. The electric conductivity $\sigma$ inside the filament could be described as:

$$
\sigma=\frac{n_{e} e^{2}}{m v_{e n}}
$$

where $m$ is the mass of electron, and $v_{e n}$ is the frequency of electron-neutral collisions. Note that $v_{e n}$ is proportional to the gas number density and the value of $v_{e n} / p$ is usually in the order of $10^{9}$ $\mathrm{sec}^{-1}$ Torr $^{-1}$. Sunka et al. measured the broadening of the $\mathrm{H} \alpha$ line profile, which is commonly used to characterize the density of plasma, reporting the electron density inside streamers during the initial phase of water breakdown, to be in the order of $10^{18} \mathrm{~cm}^{-3}$. With the room-temperature saturated water vapor pressure of 20 Torr, the electric conductivity inside the filament can be estimated to be in the order of $10^{7} \mathrm{~S} / \mathrm{m}$, a value which is comparable to those for metals. So the filament could be regarded as equipotential with the electrode, and thus could be treated as an extension of the electrode throughout the expansion. The external fluid provides drag force and constant external pressure for the development of the filament. Gravity is neglected here because the body force induced by gravity is much smaller than electric forces.

The electric field outside a slender jet can be described as if it were due to an effective linear charge density (incorporating effects of both free charge and polarization charge) of charge density $\rho_{\mathrm{e}}$ on the surface. Since the charge density in liquid can be ignored comparing with that on the filament surface, one can have the following equation for the space outside the filament by applying Laplace Equation in the radial direction:

$$
\frac{1}{r} \frac{\partial}{\partial r}\left(r \frac{\partial \Phi}{\partial r}\right)=0
$$

with boundary condition: $\left.\Phi\right|_{r=r_{0}}=\Phi_{0}$ and $\left.\Phi\right|_{r=R}=0 . R$ is the distance between anode and cathode. Since the filament could be regarded as an extension of the electrode, $R$ decreases as the streamer propagates through the gap. 
Solving the above equation with an assumption of negative discharge, the radial electric field $E_{\mathrm{r}}$ and local surface charge density $\sigma_{\mathrm{r}}$ can be written as:

$$
\begin{aligned}
& E_{r}=\frac{\partial \Phi}{\partial r}=-\frac{\Phi_{0}}{r_{0} \ln \left(R / r_{0}\right)} \\
& \rho_{e r}=\varepsilon E_{r_{0}}=-\varepsilon_{r} \varepsilon_{0} \frac{\Phi_{0}}{r_{0} \ln \left(R / r_{0}\right)}
\end{aligned}
$$

There is no analytical solution for the electric field at the hemispherical tip of the filament. A frequently used approximation is $\mathrm{E}_{\mathrm{z}} \approx \Phi_{0} / \mathrm{r}_{0}$. Here the equation for the electric field at the tip of a needle in a needle-to-plane geometry developed by Lama \& Gallo (1977) was used:

$$
E_{z}=-\frac{2 \Phi_{0}}{r_{0} \ln \left(4 R / r_{0}\right)}
$$

Similarly, the local charge density at the tip is:

$$
\rho_{e z}=\varepsilon E_{z}=-\varepsilon_{r} \varepsilon_{0} \frac{2 \Phi_{0}}{r_{0} \ln \left(4 R / r_{0}\right)}
$$

From Equations (5 -24) - (5 -27), one can conclude that the radial direction electrostatic pressure $\mathrm{E} \cdot \sigma$ exerted on the side wall of the streamer was weaker than the axial direction electrostatic pressure on the tip. Note that both electrostatic pressures were roughly inversely proportional to $r_{0}^{2}$, meaning that at the initial stage of the filament growth when $r_{0}$ is small, the electrostatic forces on both directions were strong and the filament would grow both axially and radially. A direct consequence of both the axial and radial expansions of the streamer channel is the launching of compression waves into adjacent liquids (An, Baumung, and Bluhm, 2007). At some critical point, the electrostatic force would reach a balance with hydrodynamic resistance acting on the surface in the radial direction first, while the filament continued to grow in the axial direction. 


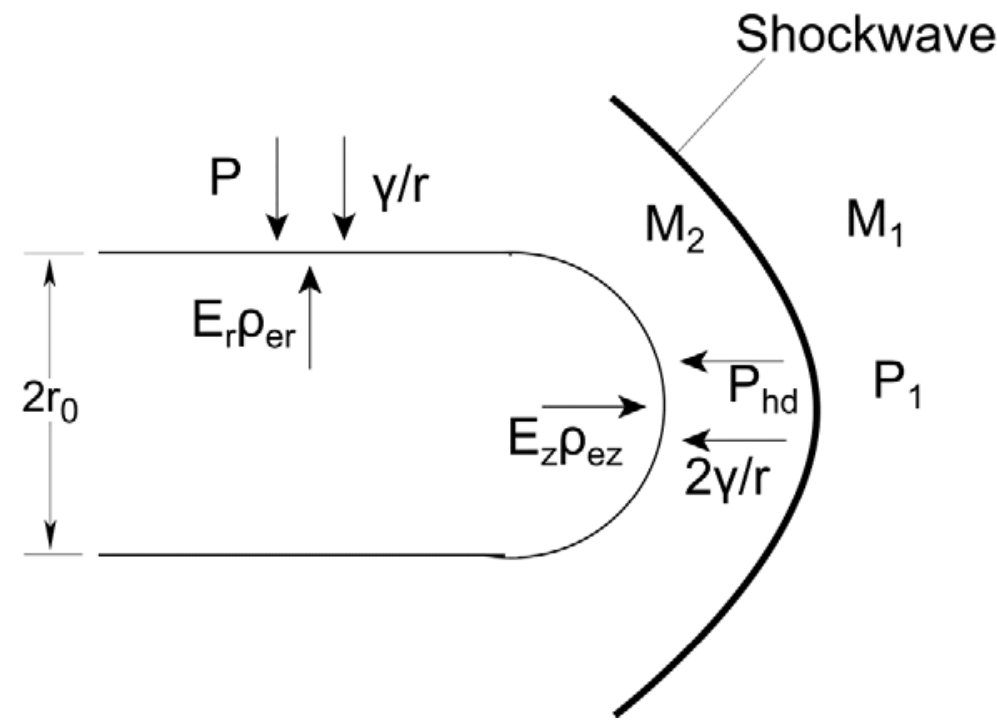

Figure 5 -12 Force balance for the present electrostatic model

Experimentally recorded propagation speeds of the filaments varied depending on the measurement techniques, ranging from a few kilometers to one hundred kilometers per second. In spite of the discrepancy observed by different groups, the propagation was clearly in the supersonic regime, and the formation of shockwaves had to be taken into consideration (see Figure 5 -12). The drag force on the tip of the streamer, which is a stagnation point, equals to the force produced by the total hydrodynamic pressure:

$$
\mathrm{P}_{\mathrm{hd}}=\mathrm{P}_{1}\left(\frac{2 \alpha}{\alpha+1} \mathrm{M}_{1}^{2}-\frac{\alpha-1}{\alpha+1}\right)+\frac{1}{2} \rho\left(\mathrm{CM}_{2}\right)^{2}
$$

where $P_{1}$ is ambient pressure, $\mathrm{P}_{1} \cdot\left(\frac{2 \alpha}{\alpha+1} \mathrm{M}_{1}^{2}-\frac{\alpha-1}{\alpha+1}\right)$ is the pressure behind shock front, $\alpha$ is the specific heat ratio of water, $M_{1}$ is the Mach number of streamer, $M_{2}$ is the Mach number after the shock front, and $C$ is the speed of sound in liquid. The relationship between $M_{1}$ and $M_{2}$ can be written as (White, 2006):

$$
\mathrm{M}_{2}^{2}=\frac{(\alpha-1) \mathrm{M}_{1}^{2}+2}{2 \alpha \mathrm{M}_{1}^{2}+1-\alpha}
$$

Equating the hydrodynamic pressure to the sum of the electrostatic pressure and the pressure produced by surface tension at the tip can give the following equation for streamer propagation:

$$
4 \varepsilon_{r} \varepsilon_{0} \frac{\Phi_{0}^{2}}{\mathrm{r}_{0}^{2} \ln ^{2}\left(4 \mathrm{R} / \mathrm{r}_{0}\right)}=\mathrm{P}_{1}\left(\frac{2 \alpha}{\alpha+1} \mathrm{M}_{1}^{2}-\frac{\alpha-1}{\alpha+1}\right)+\frac{1}{2} \rho\left(\mathrm{CM}_{2}\right)^{2}+\frac{2 \gamma}{\mathrm{r}_{0}}
$$


The balance between the electrostatic force and the force produced by the total hydrodynamic pressure in the radial direction can be given as:

$$
\varepsilon_{r} \varepsilon_{0} \frac{\Phi_{0}^{2}}{\mathrm{r}_{0}^{2} \ln ^{2}\left(\mathrm{R} / \mathrm{r}_{0}\right)}=\mathrm{P}_{1}+\frac{1}{2} \rho\left(\mathrm{CM}_{2}\right)^{2}+\frac{\gamma}{\mathrm{r}_{0}}
$$

Note that there are three unknowns, $\mathrm{M}_{1}, \mathrm{M}_{2}$ and $\mathrm{r}_{0}$, in the above equations. So it is possible to solve Equations (5 -28), (5 -29) and (5 -30) simultaneously, when the applied voltage $\Phi_{0}$ and the inter-electrode distance $\mathrm{R}$ are specified.

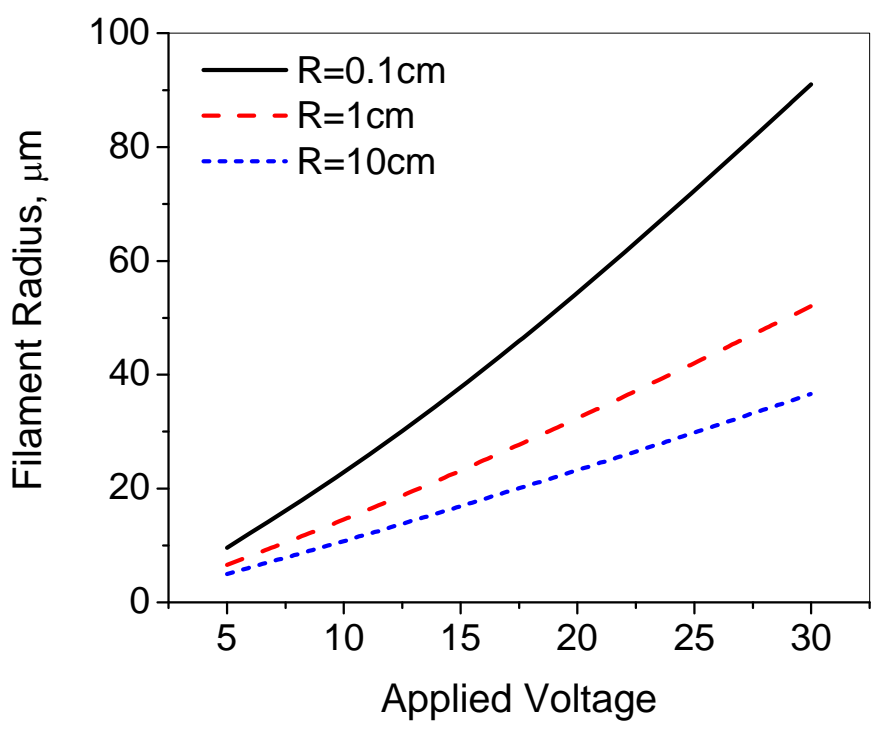

Figure $5-13$. Variations of filament radius as a function of applied voltage and inter-electrode distance

To demonstrate the validity of the present model, the filament radius predicted by the model is shown in Figure 5 -13. For a typical inter-electrode distance of $1 \mathrm{~cm}$, the filament radius increased from $3 \mu \mathrm{m}$ to $50 \mu \mathrm{m}$ as the applied voltage rose from $5 \mathrm{kV}$ to $30 \mathrm{kV}$. The value was comparable to typical experimental values. For example, An et al. (2007) reported that the light emission from the discharge was restricted to a channel of $100 \mu \mathrm{m}$ diameter, indicating the interaction of charged particles in the region.

Figure $5-14$ shows the filament propagation speed as a function of $\Phi_{0}$ and $\mathrm{R}$. The calculated propagation speed from the present model was around $15 \mathrm{~km} / \mathrm{s}$, which was higher than the primary streamer speed but lower than the secondary streamer speed reported by An et al. (2007). The Mach number increased moderately with the applied voltage, a phenomenon which is understandable from the point of view of energy conservation. The streamer 
propagation velocity was relatively independent of the inter-electrode distance. For an applied voltage of $30 \mathrm{kV}$, the Mach number increased from 11.2 to 12.3 when inter-electrode distance decreased from $10 \mathrm{~cm}$ to $0.1 \mathrm{~cm}$. This is consistent with the known property of negative streamers as the previous experiment showed that for a given voltage the propagation velocity was relatively constant as the streamer crossed the gap, and while it increased as the streamer approached the plane electrode. This phenomenon could be understood by Equation 5 -24: the inter-electrode distance $\mathrm{R}$ was decreased with the propagation of the streamers; as a result the electric field at the tip of the streamer was increased, leading to a higher propagation speed. However, the amount of the increase in the electric field would not be significant because of the natural logarithm in the equation.

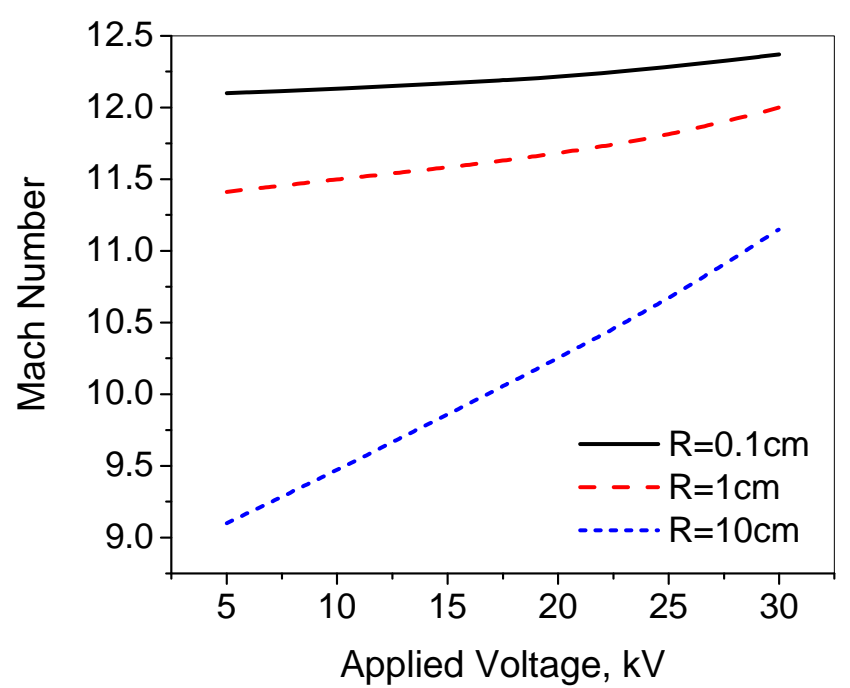

Figure 5 -14. Variations of the Mach number of streamer as a function of applied voltage and inter-electrode distance

\subsubsection{Thermal Mechanism}

In the electrostatic model described above, it was assumed that the translational temperature inside the streamer was low, and the electrostatic force was the only driving force for the growth of the filament. The assumption was valid only at the initial stage of the filament development, as the temperature will keep rising as the molecules gain more energy through electron-neutral collisions. The heating time, $\tau$, is approximately $\tau=\tau_{\mathrm{en}}+\tau_{\mathrm{vt}}$, where $\tau_{\mathrm{en}}$ is the time for electron-neutral excitation, and $\tau_{\mathrm{vt}}$ is the time for vibrational-translational (v-t) relaxation. For electron-neutral excitation, $\tau_{\mathrm{en}}=1 / v_{\mathrm{en}}=1 /\left(\mathrm{n}_{\mathrm{e}} \cdot \mathrm{k}_{\mathrm{en}}\right)$, where $v_{\mathrm{en}}$ is the electronneutral non-elastic collision frequency, $\mathrm{n}_{\mathrm{e}}$ is the electron density, and $\mathrm{k}_{\mathrm{en}}$ is the rate constant for electron-neutral collisions. $\mathrm{k}_{\mathrm{en}}$ can be expressed as $\mathrm{k}_{\mathrm{en}}=\sigma_{\mathrm{en}} \mathrm{v}_{\mathrm{te}}$, where $\sigma_{\mathrm{en}}$ is the cross section for vibrational excitation of $\mathrm{H}_{2} \mathrm{O}$ molecules by electron impact and $\mathrm{v}_{\mathrm{te}}$ is the electron thermal velocity. 
For electrons with an average energy of $1 \mathrm{eV}$, the cross section for vibrational excitation is about $\sigma=10^{-17} \mathrm{~cm}^{2} . \mathrm{k}_{\mathrm{en}}$ is thus about $10^{-8} \mathrm{~cm}^{3} / \mathrm{s}$ as is typical $\left(\mathrm{v}_{\mathrm{te}}=6 \times 10^{7} \mathrm{~cm} / \mathrm{s}\right)$. Spectroscopic measurements indicated that the stark broadening of $\mathrm{H} \alpha$ line corresponded to an electron density of about $10^{18} \mathrm{~cm}^{-3}$ at a quasi-equilibrium state. Thus the typical electron-neutral excitation time can be estimated to be in the order of a few nanoseconds. For the v-t relaxation, $\tau_{v t}=1 /\left(n_{v} \cdot k_{v t}\right)$, where $\mathrm{n}_{\mathrm{v}}$ is the density of vibrational excited molecules, $\mathrm{k}_{\mathrm{vt}}$ is the $\mathrm{v}-\mathrm{t}$ relaxation rate coefficient. For water molecules at the room temperature, $\mathrm{k}_{\mathrm{vt}}$ is about $3 \times 10^{-12} \mathrm{~cm}^{3} / \mathrm{s}$ (Fridman and Kennedy, 2006). Assuming that $\mathrm{n}_{\mathrm{v}}$ is in the same order with electron density, $\tau_{\mathrm{vt}}$ could be estimated to be in the order of several hundred nanoseconds, suggesting that heating can take place inside the filaments under sub-microsecond time scale due to the energy transfer from the electrons to the translational energy of the water molecules, and furthermore the propagation of the streamers could be caused by the continuous evaporation of water molecules at the tip. Here the energy dissipation was not considered, and the actual heating time might be longer, but still the local heating mechanism under the sub-microsecond time regime seems possible.

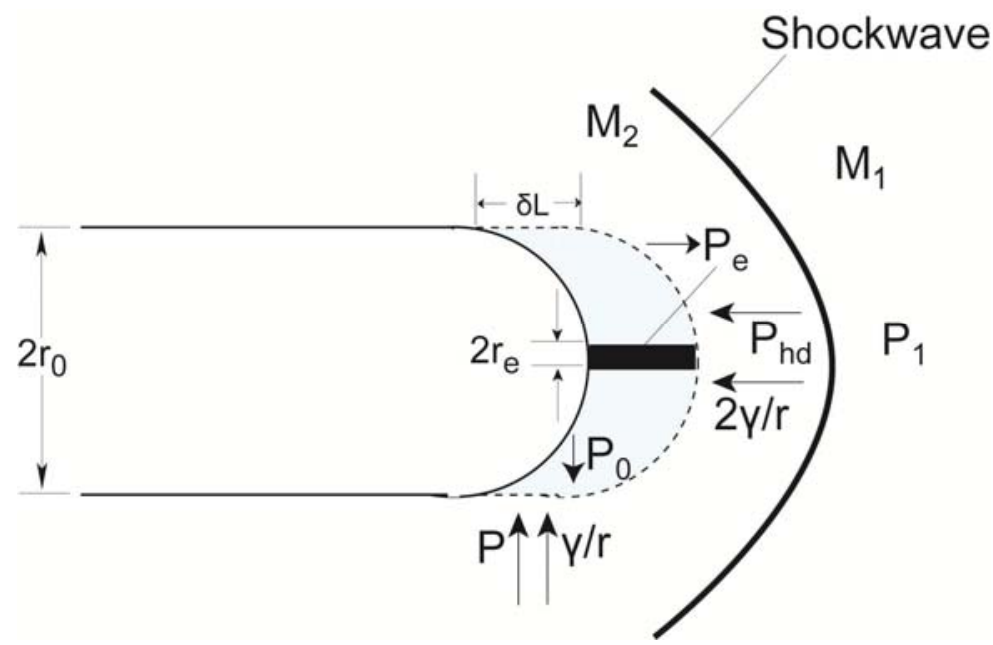

Figure 5 -15 Force balance for the present thermal model

To quantify the process described above, it was assumed that a small cylindrical portion of water evaporated at the tip of the streamer during time $\Delta t$ so that the length of the streamer grew from $\mathrm{L}$ to $\mathrm{L}+\delta \mathrm{L}$, as shown in Figure $5-15$. The diameter of the evaporated water cylinder was assumed to be $2 r_{e}$. There was no definitive value for pressure $\mathrm{P}_{\mathrm{e}}$ inside the small vaporized portion given the extremely high temperature. However $\mathrm{P}_{\mathrm{e}}$ could be estimated to be in the order of $1000 \mathrm{~atm}$ because of the density difference between liquid water and vapor. Such a high pressure could provide the driving force needed for the growth of the filament. As in the previous section, one can get the force balance along the axial direction at the tip of the filament assuming a steady state condition as: 


$$
P_{1}\left(\frac{2 \mathrm{k}}{\mathrm{k}+1} \mathrm{M}_{1}^{2}-\frac{\mathrm{k}-1}{\mathrm{k}+1}\right)+\frac{1}{2} \rho\left(\mathrm{CM}_{2}\right)^{2}+\frac{2 \gamma}{\mathrm{r}_{e}}=\mathrm{P}_{e}(5-31)
$$

The energy required for the evaporation of water can be calculated as

$$
\mathrm{E}_{e}=\rho \mathrm{V}_{e}\left(\mathrm{c}_{\mathrm{p}} \Delta \mathrm{T}+\Delta_{\mathrm{v}} \mathrm{H}\right)
$$

where $\rho$ is the density of water, $V_{e}$ is the volume of evaporated water, $C_{p}$ is the specific heat of water. $V_{e}$ can be written as

$$
\mathrm{V}_{e}=\pi \mathrm{r}_{e}^{2} \cdot \delta \mathrm{L}
$$

After evaporation, the overheated and over-pressured water vapor will expand radially, while satisfying the force balance along the axial direction, until it reaches equilibrium with the outside hydrodynamic pressure. The process could be regarded as adiabatic under a submicrosecond time scale, and thus one can have the following equations:

$$
\begin{aligned}
& \mathrm{P}_{e} \mathrm{~V}_{e}^{\alpha_{s}}=\mathrm{P}_{0} \mathrm{~V}_{0}^{\alpha_{s}} \\
& \frac{\mathrm{P}_{0}}{\mathrm{P}_{\mathrm{e}}}=\left[\frac{\varepsilon_{r} \varepsilon_{0} \Phi_{0}^{2}}{\rho \mathrm{r}_{0}\left(\mathrm{c}_{\mathrm{p}} \Delta \mathrm{T}+\Delta \mathrm{H}_{1-\mathrm{g}}\right) \ln \left(\mathrm{R} / \mathrm{r}_{0}\right)}\right]^{\alpha_{s}}
\end{aligned}
$$

where $P_{0}$ and $V_{0}$ are the pressure and volume respectively of the water vapor after the expansion, $r_{0}$ is the radius of the filament after expansion, $\alpha_{s}$ is the specific heat ratio of the water vapor. The force produced by $P_{0}$ should be in balance with the forces created by both surface tension and total environmental hydrodynamic pressure as given below:

$$
\mathrm{P}_{0}=\mathrm{P}_{1}+\frac{1}{2} \rho\left(\mathrm{CM}_{2}\right)^{2}+\frac{\lambda}{\mathrm{r}_{0}}
$$

Another set of equations can be obtained through the consideration of energy conservation. The energy required to vaporize water was the electric energy provided by the power supply. If the entire filament was viewed as a capacitor with capacitance $C$, the required energy could be calculated as:

$$
\mathrm{E}=\frac{\mathrm{C} \Phi_{0}^{2}}{2}
$$

The capacitance of the cylindrical filament is: 


$$
\mathrm{C}=2 \pi \varepsilon \varepsilon_{0} \mathrm{r}_{0} \mathrm{~L} / \ln \left(\mathrm{R} / \mathrm{r}_{0}\right)
$$

So the energy change required to extend the length by $\delta L$ becomes:

$$
\delta \mathrm{E}=\pi \varepsilon \varepsilon_{0} \mathrm{r}_{0} \delta \mathrm{L} \Phi_{0}^{2} / \ln \left(\mathrm{R} / \mathrm{r}_{0}\right)
$$

By equating $\delta E$ to $E_{e}$, one has

$$
r_{e}=\sqrt{\frac{\varepsilon \varepsilon_{0} r_{0} \Phi_{0}^{2}}{\rho\left(c_{p} \Delta T+\Delta H_{1-g}\right) \ln \left(R / r_{0}\right)}}
$$

Assuming $\alpha \approx 1$ due to the low compressibility of water, and rearranging Equations (5 -35), (5 42), (5 -36), (5 -37) and (5 -41) to eliminate $\mathrm{M}_{2}, \mathrm{r}_{\mathrm{e}}$ and $\mathrm{P}_{\mathrm{g}}$, one can get a set of equations about $\mathrm{M}_{1}$ and $\mathrm{r}_{0}$ as follows:

$$
\begin{gathered}
P_{1} M_{1}^{2}+\frac{\rho C^{2}}{2 M_{1}^{2}}-P_{e}+2 \gamma \sqrt{\frac{\rho\left(\mathrm{c}_{\mathrm{p}} \Delta \mathrm{T}+\Delta \mathrm{H}_{1-\mathrm{g}}\right) \ln \left(R / r_{0}\right)}{\varepsilon_{r} \varepsilon_{0} r_{0} \Phi_{0}^{2}}}=0 \\
P_{e}\left[\frac{\varepsilon_{r} \varepsilon_{0} \Phi_{0}^{2}}{\rho r_{0}\left(\mathrm{c}_{\mathrm{p}} \Delta \mathrm{T}+\Delta \mathrm{H}_{1-\mathrm{g}}\right) \ln \left(R / r_{0}\right)}\right]^{k_{s}}-P_{1}-\frac{\rho C^{2}}{2 M_{1}^{2}}-\gamma \sqrt{\frac{\rho\left(\mathrm{c}_{\mathrm{p}} \Delta \mathrm{T}+\Delta \mathrm{H}_{1-\mathrm{g}}\right) \ln \left(R / r_{0}\right)}{\varepsilon_{r} \varepsilon_{0} r_{0} \Phi_{0}^{2}}}=0
\end{gathered}
$$

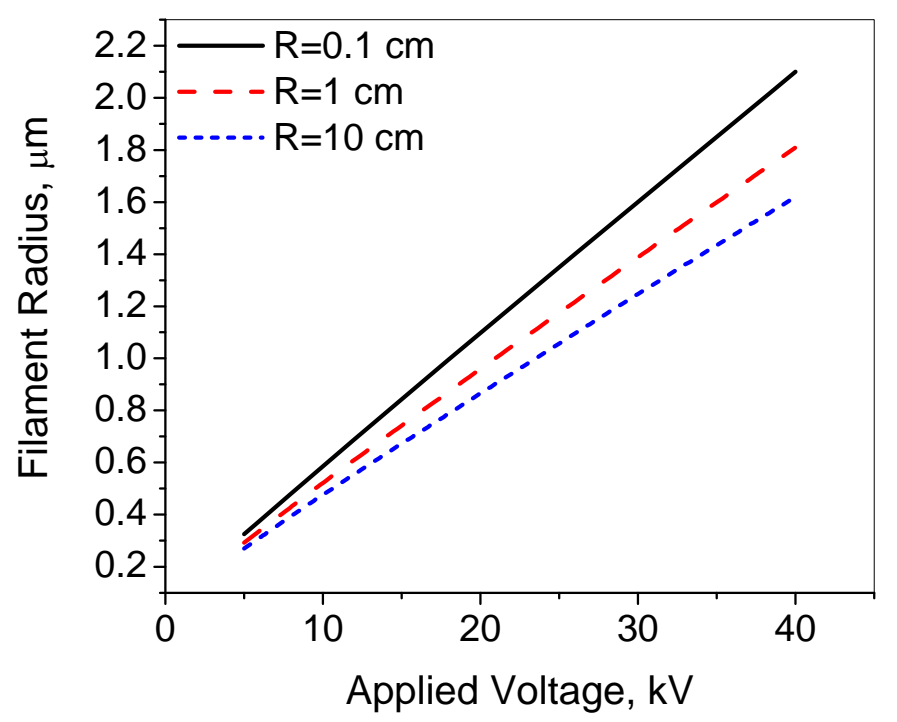

Figure 5 -16. Variations of filament radius as a function of applied voltage and inter-electrode distance 


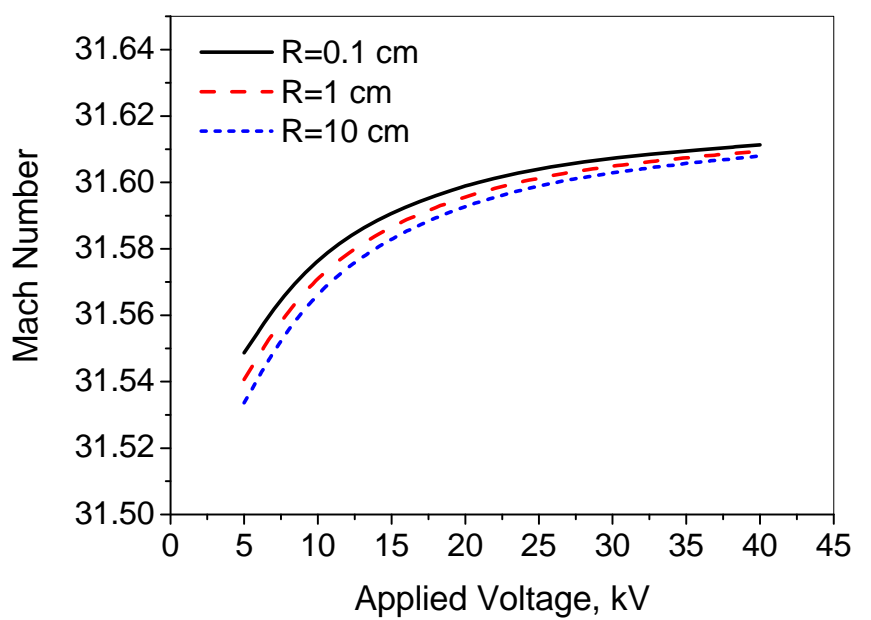

Figure $5-17$. Variations of the Mach number of streamer as a function of applied voltage and inter-electrode distance

For water vapor, $\alpha_{s}$ can be assumed to be 1.3 (Parry et al., 2008). For high-temperature underwater discharges, the translational plasma temperature was measured to be between $4000 \mathrm{~K} \sim 6500 \mathrm{~K}$ (Lange and Huczko, 2004). An average value of $5000 \mathrm{~K}$ was used for $\Delta \mathrm{T}$ in the present study. Figure 5-17 shows the Mach number of filament propagation, $\mathrm{M}_{1}$, as a function of $\Phi_{0}$ and R. The propagation velocity was about $50 \mathrm{~km} / \mathrm{s}$, which was higher than the secondary streamer velocity of $25 \mathrm{~km} / \mathrm{s}$ reported by Baumung and Bluhm (2007), but lower than the value of $200 \mathrm{~km} / \mathrm{s}$ reported by Woodworth et al. (Woodworth et al., 2004). The discrepancy in the two measurements probably came from the different techniques used for the velocity measurements. The value of $M_{1}$ remained constant for various values of $\Phi_{0}$ and $R$, indicating that the propagation velocity of the streamers was independent of either the applied voltage or interelectrode distance. Similar phenomenon was observed previously, where the propagation velocity of secondary streamers was constant over a wide voltage range. Figure 5-16 shows the filament radius as a function of $\Phi_{0}$ and $\mathrm{R}$. The radius increased slightly as the streamers approached the other electrode, while it decreased almost linearly as the applied voltage dropped. The absolute value of $r_{0}$ was about one order smaller than that obtained from the electrostatic model. This could be understood if one considers the energy requirements for the two mechanisms. For the evaporation of water, the energy needed to break the hydrogen bonds between water molecules should be much greater than that required to displace the same volume of water.

The different models based on the electrostatic force and evaporation gave different results of the streamer propagation speed and filament radius. The electrostatic model showed streamers with a larger radius and a lower Mach number, while the thermal model demonstrated that the streamers could move much faster but were thinner than those determined from the electrostatic model. The different findings from the two models led us to postulate that different 
mechanisms might be associated with the different modes of the streamer propagation. At the initial primary streamer mode before any significant heat was generated, the electrostatic force might have played a major role. The appearance of the secondary streamer required more time, during which the electron energy could be transferred to translational energy of water molecules and subsequently evaporation become the dominant force to drive the filament to move forward. The transition time between the primary and secondary streamers was in the order of $100 \mathrm{~ns}$, a value which is in accordance with the heating time as estimated above.

\subsection{Stability Analysis of the Streamers}

The breakdown process is usually characterized by two features: an initial development of thin discharge channels and a subsequent branching of these channels into complicated "bushlike" patterns. Apparently, the branching process is associated with the instability of the filament. In this section the linear stability analysis of axisymmetric perturbation of a filament surface with a certain electric charge density is presented. As long as the wavelength of the perturbation is much smaller than the length of the filament, the stability characteristics can be approximated by considering perturbations to a charged cylinder of constant radius as shown in Figure 5 -18. The peak-to-peak amplitude and wave number of the disturbance are $h$ and $k$, respectively. $H$ is the depth of wave influence, and $u$ is the velocity of liquid relative to the disturbance. Then the surface of the perturbation can be represented by the following equation:

$$
\mathrm{r}=\mathrm{r}_{0}+\frac{\mathrm{h}}{2} \exp (\mathrm{ikz}+\omega \mathrm{t})
$$

where $\omega$ is the oscillation frequency of the instability. To analyze the linear stability, the disturbance of the local electrostatic force, surface tension and hydrodynamic pressure were considered following a geometrical perturbation. Generally, the surface tension tends to minimize the surface area and subsequently stabilize the disturbance, while the local enhancement of the electrostatic force tended to push the disturbance to grow. In the reference frame that moves together with the tip of filament, the effects of these three forces were considered separately for the pressure balance between the crest and trough along the stream line (see Figure 5 -18). 


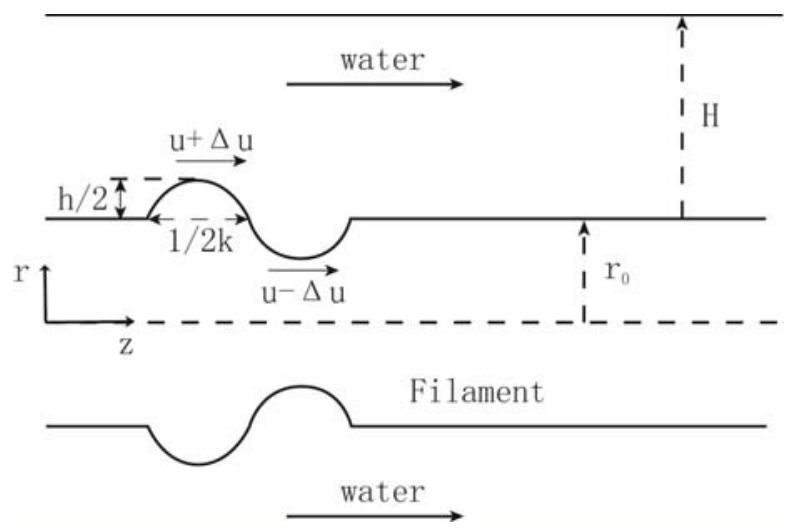

Figure 5 -18. Schematic diagram of disturbance at the surface of filament

\subsubsection{Electrostatic Pressure}

According to Equations (5 -24) - (5 -27), the electrostatic pressure is proportional to the square of the local curvature of the interface, which is different at the crest and trough of the perturbation. Thus the electrostatic pressures at the crest and trough, $P_{\mathrm{E}, \mathrm{c}}$ and $P_{\mathrm{E}, \mathrm{t}}$ become respectively,

$$
\mathrm{P}_{\mathrm{E}, \mathrm{c}}=\varepsilon_{\mathrm{r}} \varepsilon_{0} \Phi_{0}^{2} \frac{\chi_{\mathrm{c}}^{2}}{4}
$$

and

$$
\mathrm{P}_{\mathrm{E}, \mathrm{t}}=\varepsilon_{\mathrm{r}} \varepsilon_{0} \Phi_{0}^{2} \frac{\chi_{\mathrm{t}}^{2}}{4}
$$

where $\varepsilon_{r}$ is the relative permittivity of water, and $\chi_{c}$ and $\chi_{\mathrm{t}}$ are the mean curvatures at the crest and trough, respectively. The expression for the mean curvature can be written as:

$$
\chi=\frac{1}{\mathrm{r} \sqrt{1+\left(\partial_{z} r\right)^{2}}}-\frac{\partial_{z}\left(\partial_{z} r\right)}{\left(1+\left(\partial_{z} r\right)^{2}\right)^{3 / 2}}=\frac{1}{r}-\partial_{z}\left(\partial_{z} r\right)
$$

Substituting Equation (5 -44) into Equation (5 -47), one can get expressions for $M_{\mathrm{c}}$ and $M_{\mathrm{t}}$ :

$$
\begin{aligned}
& \chi_{\mathrm{c}}=\frac{1}{\mathrm{r}_{0}+\mathrm{h} / 2}+\frac{\mathrm{h}}{2} \mathrm{k}^{2} \\
& \chi_{\mathrm{c}}=\frac{1}{\mathrm{r}_{0}+\mathrm{h} / 2}-\frac{\mathrm{h}}{2} \mathrm{k}^{2}
\end{aligned}
$$

Subsequently, $P_{\mathrm{E}, \mathrm{c}}$ and $P_{\mathrm{E}, \mathrm{t}}$ can be written as: 


$$
\begin{aligned}
& \mathrm{P}_{\mathrm{E}, \mathrm{c}}=\varepsilon_{\mathrm{r}} \varepsilon_{0} \Phi_{0}^{2} \frac{\chi_{\mathrm{c}}^{2}}{4}=\varepsilon_{\mathrm{r}} \varepsilon_{0} \Phi_{0}^{2}\left(\frac{1}{\left(2 \mathrm{r}_{0}+\mathrm{h}\right)^{2}}+\frac{\mathrm{hk}^{2}}{2\left(2 \mathrm{r}_{0}+\mathrm{h}\right)}\right) \\
& \mathrm{P}_{\mathrm{E}, \mathrm{t}}=\varepsilon_{\mathrm{r}} \varepsilon_{0} \Phi_{0}^{2} \frac{\chi_{\mathrm{c}}^{2}}{4}=\varepsilon_{\mathrm{r}} \varepsilon_{0} \Phi_{0}^{2}\left(\frac{1}{\left(2 \mathrm{r}_{0}-\mathrm{h}\right)^{2}}-\frac{\mathrm{hk}^{2}}{2\left(2 \mathrm{r}_{0}-\mathrm{h}\right)}\right)
\end{aligned}
$$

Thus, the electrostatic pressure difference between the crest and trough becomes:

$$
\Delta P_{E}=P_{E, c}-P_{E, t}=-\frac{\varepsilon_{\mathrm{r}} \varepsilon_{0} \Phi_{0}^{2} \mathrm{~h}}{2 r_{0}^{3}}+\frac{\varepsilon_{\mathrm{r}} \varepsilon_{0} \Phi_{0}^{2} \mathrm{hk}^{2}}{2 r_{0}}
$$

\subsubsection{Surface Tension}

The pressures due to the surface tension across the interface at the crest and trough can be written as:

$$
\mathrm{P}_{\mathrm{T}, \mathrm{c}}=\gamma \chi_{\mathrm{c}}=\gamma\left(\frac{1}{\mathrm{r}_{0}+\mathrm{h} / 2}+\frac{\mathrm{h}}{2} \mathrm{k}^{2}\right)
$$

and

$$
\mathrm{P}_{\mathrm{T}, \mathrm{t}}=\gamma \chi_{\mathrm{t}}=\gamma\left(\frac{1}{\mathrm{r}_{0}-\mathrm{h} / 2}-\frac{\mathrm{h}}{2} \mathrm{k}^{2}\right)
$$

Thus the pressure difference due to surface tension between the cress and trough becomes:

$$
\Delta P_{T}=P_{T, c}-P_{T, t}=-\frac{\gamma \mathrm{h}}{r_{0}^{2}-h^{2} / 4}+\gamma \mathrm{hk}^{2}
$$

Since $r_{0}>>h$, the above equation can be simplified as:

$$
\Delta P_{T}=-\frac{\gamma \mathrm{h}}{r_{0}^{2}}+\gamma \mathrm{hk}^{2}
$$

\subsubsection{Hydrodynamic Pressure}

When there is a disturbance on the interface of the filament, the flow speed of liquid will be perturbed in the depth of wave influence, inducing a hydrodynamic pressure difference $\Delta \mathrm{P}_{\mathrm{H}}$ between the crest and trough:

$$
\Delta P_{H D}=\frac{1}{2} \rho\left(u+\frac{\Delta u}{2}\right)^{2}-\frac{1}{2} \rho\left(u-\frac{\Delta u}{2}\right)^{2}=\rho u \Delta u(5-56)
$$


where $\Delta u / 2$ is the perturbation in the flow speed caused by the shape of the wave. The dynamic pressure is related to the flow speed through Bernoulli's equation. The pressure difference from the electrostatic force and dynamic effect of the flow has the opposite sign due to the surface tension. For a balance between two kinds of oppositely directed pressure differences one has:

$$
\rho u \Delta u+\frac{\gamma \mathrm{h}}{r_{0}^{2}}-\gamma \mathrm{hk}^{2}-\frac{\varepsilon_{\mathrm{r}} \varepsilon_{0} \Phi_{0}^{2} \mathrm{~h}}{2 r_{0}^{3}}+\frac{\varepsilon_{\mathrm{r}} \varepsilon_{0} \Phi_{0}^{2} \mathrm{hk}^{2}}{2 r_{0}}=0
$$

In order to solve Equation (5 -57), the perturbed flow speed $\Delta u$ must be expressed in terms of experimentally measurable quantities. The following derivation was inspired by Kenyon. Assuming that the perturbed flow speed is constant over the depth of wave influence, the mass conservation equation through vertical cross-sections between the crest and trough becomes:

$$
\left(u+\frac{\Delta u}{2}\right)\left(\mathrm{H}-\frac{\mathrm{h}}{2}\right)=\left(u-\frac{\Delta u}{2}\right)\left(\mathrm{H}+\frac{\mathrm{h}}{2}\right)
$$

where $H$ is the depth of wave influence. The above equation can be reduced to:

$$
u \cdot h=\Delta u \cdot H
$$

The theoretical expression for $H$ was given by Kenyon as

$$
H=\frac{1}{2 \pi k}
$$

Using Equations (5 -59) and (5 -60) to eliminate $H$ and $\Delta u$, Equation (5 -57) becomes:

$$
\rho \omega^{2}=k\left(\gamma \mathrm{k}^{2}+\frac{\varepsilon_{\mathrm{r}} \varepsilon_{0} \Phi_{0}^{2}}{2 r_{0}^{3}}-\frac{\gamma}{r_{0}^{2}}-\frac{\varepsilon_{\mathrm{r}} \varepsilon_{0} \Phi_{0}^{2} k^{2}}{2 r_{0}}\right)
$$

Since this is a quadratic equation, there will be two different branches of the dispersion relation, and an instability occurs if $\operatorname{Re}(\omega)>0$. The first thing to note in Equation (5-61) is that when the applied voltage $\Phi_{0}$ is equal to zero and the surface is flat, in other words, when the radius of the filament $r_{0}$ goes to infinity, the above equation reduces to $\rho u^{2}=\gamma k$, which is the equation for the classic two-dimensional Rayleigh instability. 


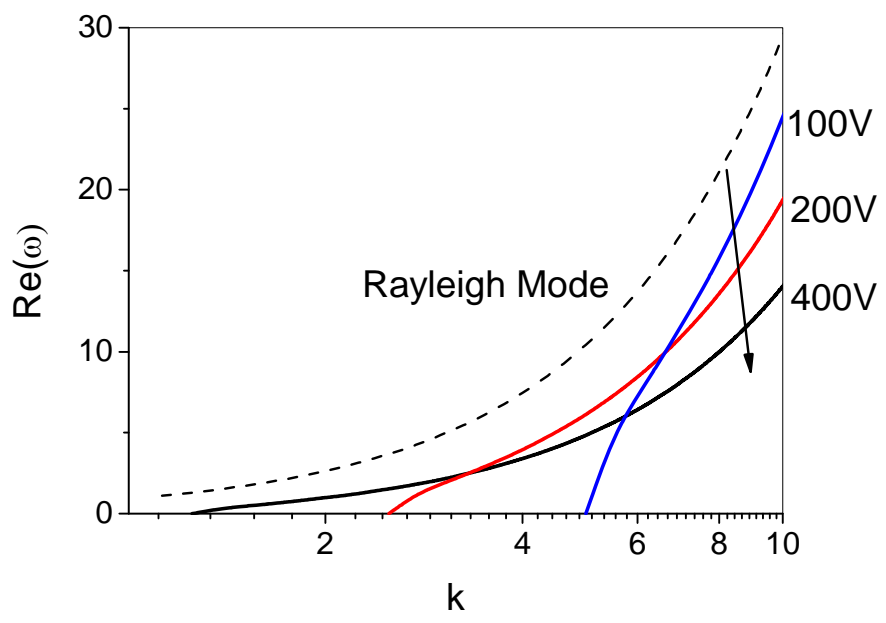

Figure 5 -19. Instability growth rate $\omega$ at low applied voltages. $\mathrm{k}$ and $\omega$ are nondimensionalized using streamer radius $\mathrm{r}_{0}$ and time scale $\mathrm{t}=\left(\mathrm{r}_{0}{ }^{3} / \gamma\right)^{1 / 2}$.

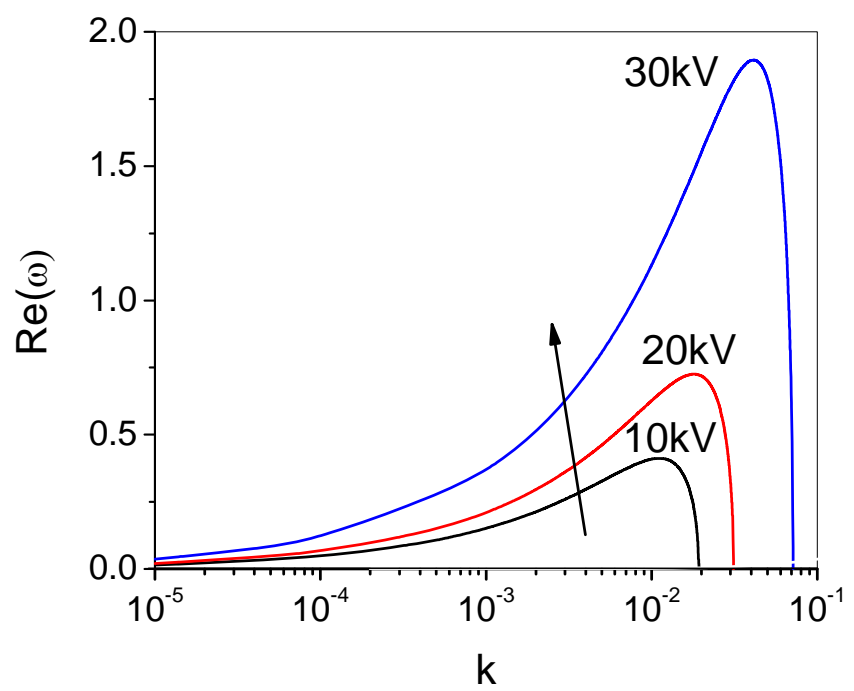

Figure 5 -20. Instability growth rate $\omega$ at high applied voltages. $\mathrm{k}$ and $\omega$ are nondimensionalized as in Figure $5-19$.

Figure $5-19$ shows the instability growth rate $\omega$ at a low applied voltage, where the process is in Rayleigh mode. The dashed line represents the classic Rayleigh instability for $\Phi_{0}=$ 0 and $\mathrm{r}_{0} \rightarrow \infty$. For $\Phi_{0} \neq 0$ and $\mathrm{r}_{0}$ is finite, instability only happens at high wave numbers. When the voltage increases under this mode, the growth rate is decreased until fully suppressed at a certain critical value. The physical explanation to this can be as follows: the Rayleigh instability occurs due to surface tension, which always acts to break a cylindrical jet into a stream of 
droplets; on the other hand, the electrostatic force, which is proportional to the square of the applied voltage, always acts on the opposite direction of the surface tension. When the applied voltage increases, the Rayleigh instability would be suppressed when the two forces are balanced.

As the voltage continues to increase, the instability enters the electrostatic mode, where the electrostatic force exceeds the force created by the surface tension and becomes the dominant force. Figure $5-20$ shows the instability growth rate $\omega$ at a high voltage. Both the growth rate and the range of wave number increase as the voltage rises. The physics of this mode is a consequence of the interaction of the electric field with the surface charge on the interface; surface tension is a parameter of less importance for this mode. The mechanism for the instability is that a perturbation in the radius of the filament induces a perturbation in the surface charge density and therefore a perturbation in the electrostatic pressure. At a high voltage, the perturbation is amplified by the fact that the electrostatic pressure $\mathrm{P}_{\mathrm{E}}$ is proportional to $\Phi^{2}$, causing the instability. In contrast to the Rayleigh mode, the instability in the electrostatic mode is unavoidable at low wave numbers (long wavelength). This may explain why the filament always tends to branch into bush-like structures.

\subsection{Nanosecond and Sub-Nanosecond Discharge in Water}

In the previous sections the initiation and propagation of electric breakdown process in liquid under microsecond high voltage excitations are described. The question appears: what would happen if the high voltage pulse duration is short enough so that no cavitation would have enough time to be formed? In the current section, the formation of plasma discharge in water under nanosecond and sub-nanosecond pulse excitations will be discussed, and the possibility to generate plasma in condensed media without density decrease will be demonstrated.

Two different pulsed power systems were used. The first power supply generates pulses with $+26 \mathrm{kV}$ pulse amplitude in 50-Ohm coaxial cable, $10 \mathrm{~ns}$ pulse duration, $0.3 \mathrm{~ns}$ rise time and $3 \mathrm{~ns}$ fall time. The second system generates $112 \mathrm{kV}$ pulses with $150 \mathrm{ps}$ rise time and duration on the half-height about 500 ps. Typical examples of pulse shapes are presented in the Figure 5 -21. 

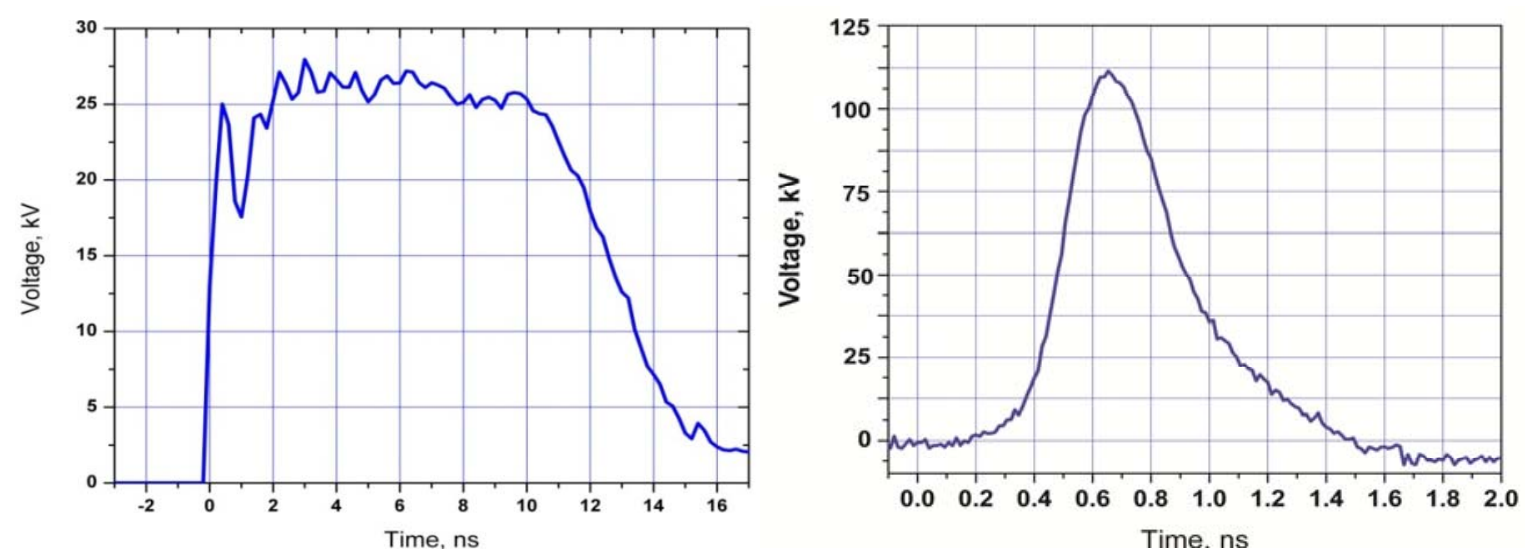

Figure 5 -21. High-voltage pulses used in experiments. a) nanosecond generator; b) subnanosecond generator.
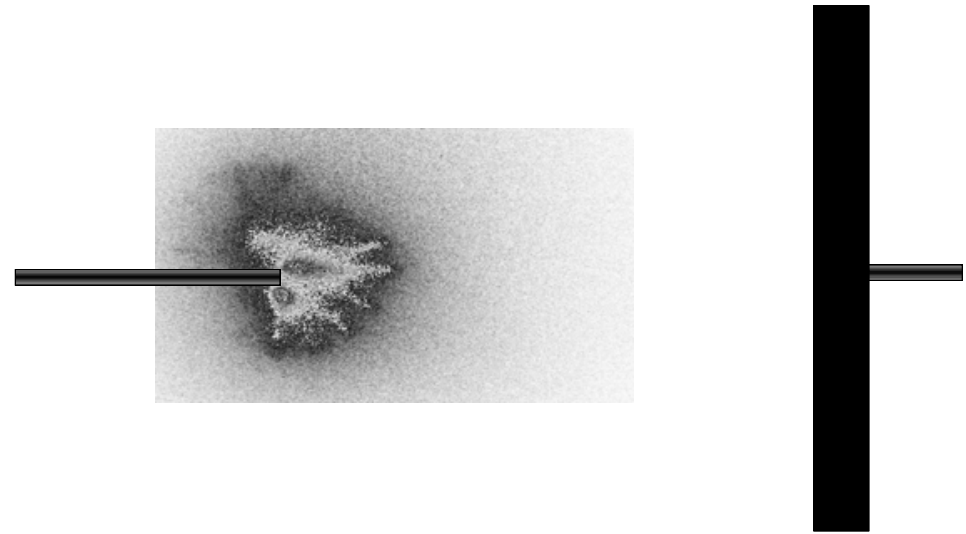

Figure 5 -22. Geometry of discharge gap and ICCD camera field of view. Distilled water, $U=$ $27 \mathrm{kV}$. Image was taken $5 \mathrm{~ns}$ after the pulse start. Camera gate was $1 \mathrm{~ns}$, field of view was $2.6 \times 1.7 \mathrm{~mm}$.

It was found that discharge in liquid water develops in picosecond time scale. Size of excited region near the tip of the high-voltage electrode was $\sim 1 \mathrm{~mm}$. The discharge has a complex multi-channel structure (Figure $5-22$ ) and this structure has changed from pulse to pulse. Thus an image accumulation leads to discharge structure elimination but gives us the possibility to trace the emission dynamics with 500-ps camera gate (Figure $5-23$ ). Figure $5-23$ demonstrates the dynamics of emission from the discharge taken with 1000 accumulations. Image overlapping removes fine discharge structure but allows better temporal resolution. It is clearly seen that the discharge emission has a typical duration very close to the camera gate (500 ps) while the emission rise time has even shorter time scale ( $\sim 250 \mathrm{ps})$. The high-voltage rise time was about $150 \mathrm{ps}$ and we can assume that the typical discharge development time is 
close to these values (between 150 and $250 \mathrm{ps).} \mathrm{Size} \mathrm{of} \mathrm{the} \mathrm{emitting} \mathrm{region} \mathrm{has} \mathrm{diameter} \mathrm{of}$ about $1 \mathrm{~mm}$.
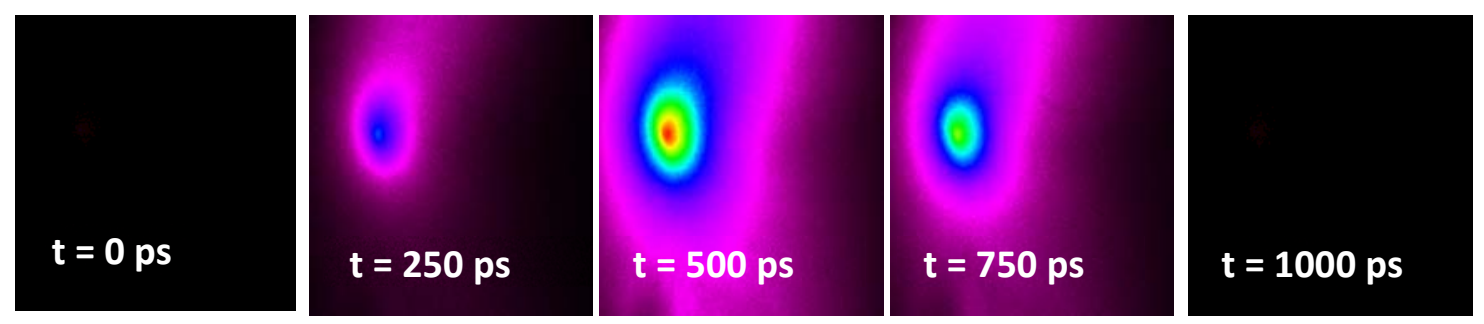

Figure 5 -23. Discharge emission dynamics. 1000 accumulations/image. Camera gate was 500 ps, $\Delta \lambda=250-750 \mathrm{~nm}$. Image size was $7 \times 4 \mathrm{~mm}$.

To analyze the spatial structure of the discharge we used longer camera gate ( $1 \mathrm{~ns})$ without signal accumulation. As expected, the temporal resolution of the image decreases and the observed emitting discharge phase becomes longer (Figure 5 -24). In exchange we have now the spatial distribution of the emission, which looks like familiar gas-phase streamer discharge.

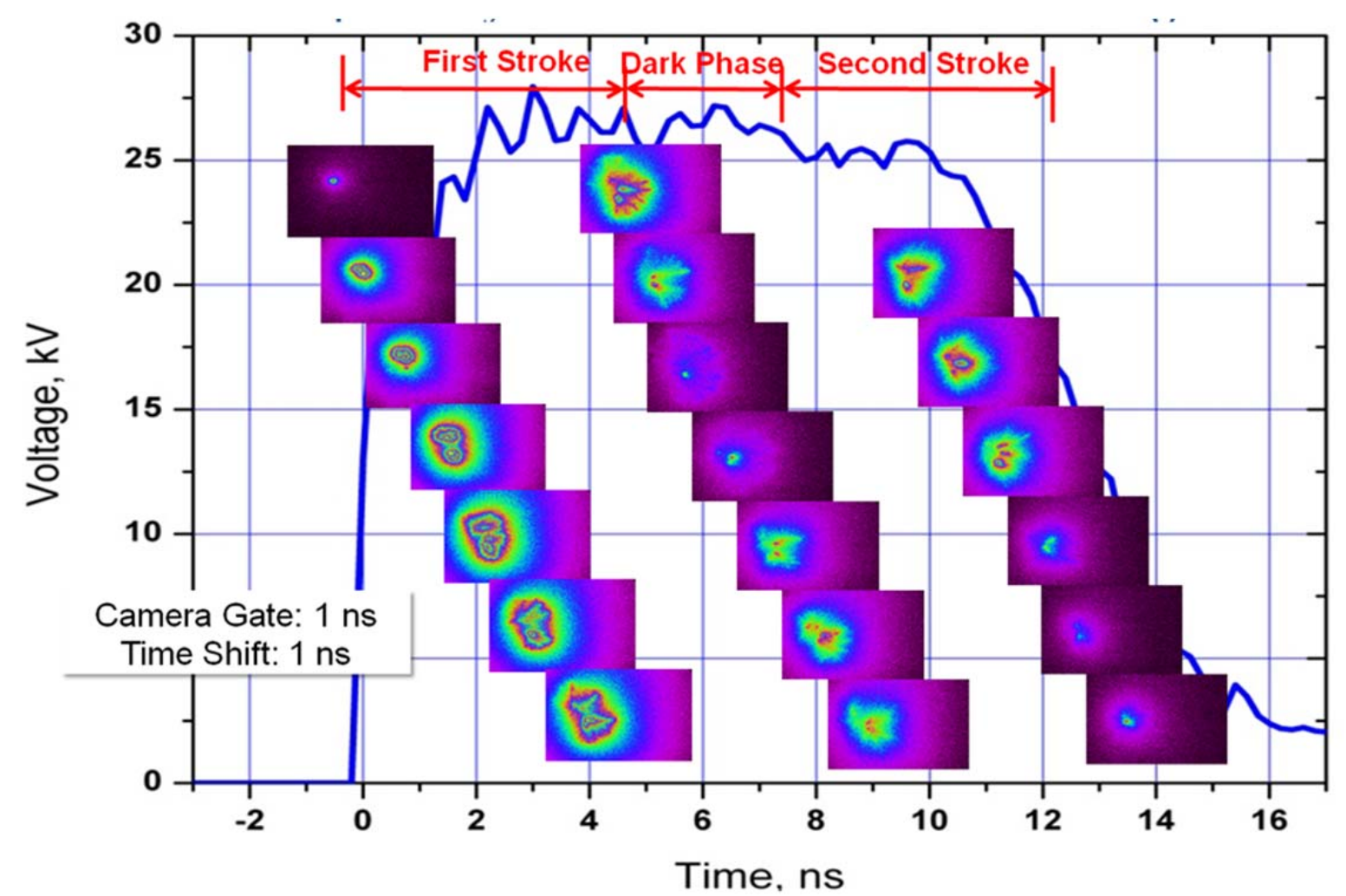

Figure 5 -24. Dynamics of discharge emission and high-voltage potential on electrode. Distilled 
water, $U=27 \mathrm{kV}$. Camera gate is $1 \mathrm{~ns}$. Spectral response $250-750 \mathrm{~nm}$.

Typical emitting channel's diameter was $\sim 50 \mu \mathrm{m}$ and propagation length was $0.5-0.6 \mathrm{~mm}$ for $U=27 \mathrm{kV}$. In the case of short rise time we observed discharge propagation with the velocity up to $2000 \mathrm{~km} / \mathrm{s}$ ( $2 \mathrm{~mm} / \mathrm{ns}$ ) during very initial stage of the discharge corresponded to the voltage rise time (Figure $5-24$ ). When voltage reaches the maximum, the discharge propagation stops and the "dark phase" appears $(t=6-9 \mathrm{~ns}$ in Figure $5-24)$. During this phase discharge cannot propagate because of space charge formation and electric field decrease. Voltage decrease leads to the second stroke formation and second emission phase (Figure 5 -24, 10-13 ns). This means that the channels lost the conductivity and the trailing edge of the nanosecond pulse generates significant electric field and excitation of the media which is comparable to the excitation corresponded to the leading edge of the pulse.

No bubbling or void formation was observed during the experiments. The discharge observed has a completely different nature from the discharges initiated by electrical pulses with a longer rise time (see, for example, Malik et al., 2005; Lukes et al., 2008; An, Baumung, and Bluhm, 2007). In the publication by An, Baumung, and Bluhm (2007) the pulses with $40 \mathrm{~ns}$ rise time at $\mathrm{U}=18 \mathrm{kV}$ demonstrate the velocity of discharge propagation about $2.5 \mathrm{~km} / \mathrm{s}$ during the initial phase of the discharge and up to $35 \mathrm{~km} / \mathrm{s}$ during second phase. Both the shadowgraph and Schlieren images suggest that the branches are of gaseous nature.

The experiments at elevated (up to $220 \mathrm{kV}$ ) voltage prove the discharge propagation in the liquid phase. The experiments were performed using ultra-short pulses with typical duration about 400 ps and rise time of 150 ps (Figure 5-21,b). Figure $5-25$ demonstrates the integral image of the discharge (camera gate was $100 \mathrm{~ns}$ ) for different voltages. It is clear that the discharge formation depends on the voltage applied. At $92 \mathrm{kV}$ discharge formation takes longer time than the pulse width and only very initial phase of the discharge formation could be observed (Figure 5 -25, a). Voltage increase elongates the plasma channels from 1-2 $\mathrm{mm}$ for $107 \mathrm{kV}$ to $6-8 \mathrm{~mm}$ for $220 \mathrm{kV}$ (Figure $5-25$, b-f). Typically we observed formation of 2-3 channels from the top and bottom edges of the electrode's tip. The length of the channels decreases gradually with the voltage decrease which for fixed pulse duration means the streamer velocity decrease. For voltage below $90 \mathrm{kV}$ discharge cannot start during 400 ps. 


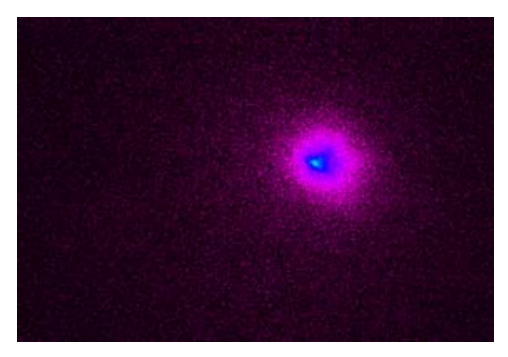

$\mathrm{U}_{\text {peak }}: 46 \mathrm{kV}$

$\mathrm{dU} / \mathrm{dt}: 0.31$

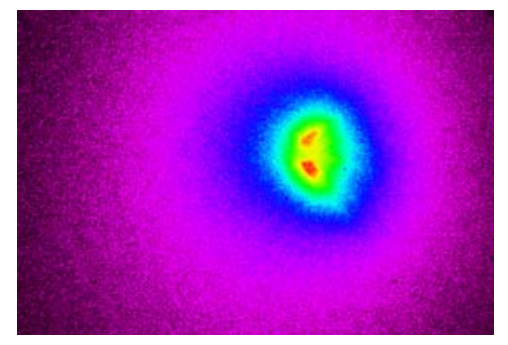

$\mathrm{U}_{\text {peak }}: 92 \mathrm{kV}$

dU/dt: 0.61

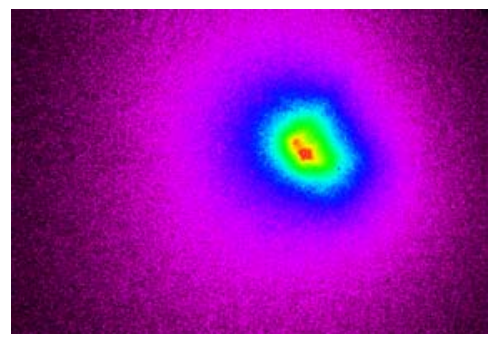

$U_{\text {peak }}: 53.5 \mathrm{kV}$

$\mathrm{dU} / \mathrm{dt}: 0.36$

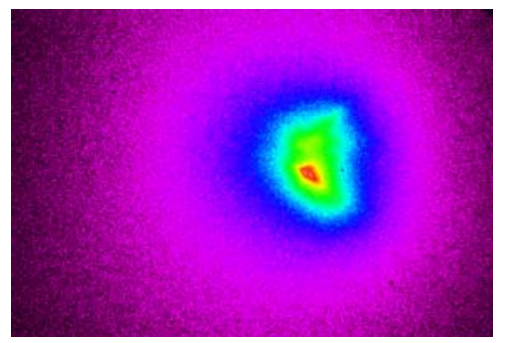

$\mathrm{U}_{\text {peak }}: 103.5 \mathrm{kV}$

dU/dt: 0.69

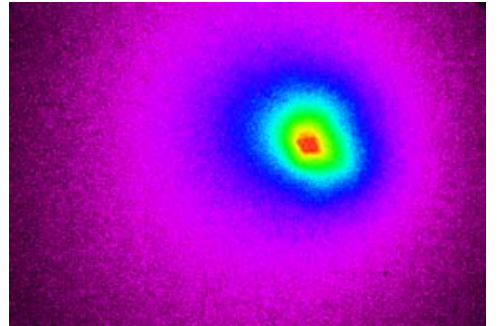

$\mathrm{U}_{\text {peak }}: 80.5 \mathrm{kV}$

dU/dt: 0.54

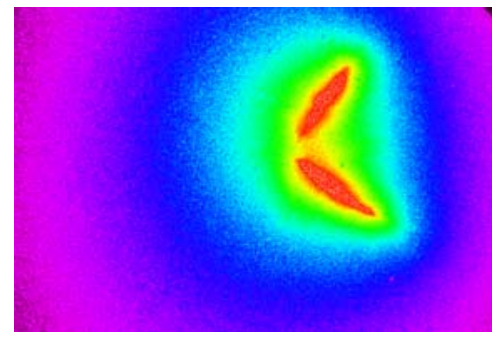

$\mathrm{U}_{\text {peak }}: 110 \mathrm{kV}$

dU/dt: 0.73

Figure 5 -25. Discharge development for different voltage. Pulse width is 400 ps. ICCD camera gate is $100 \mathrm{~ns}$.

As in the case with 10-ns pulses no bubble formation was observed. Figure 5 -26 shows the dynamics of the discharge formation for $U=224 \mathrm{kV}$. The $\Lambda$-shaped pulse has no plateau and the dark phase of the discharge did not appear (Figure 5 -26). Discharge development takes an extremely short time (in 100-200 ps plasma channels reach the length of 5-8 mm, diameter $100 \mu \mathrm{m})$. 


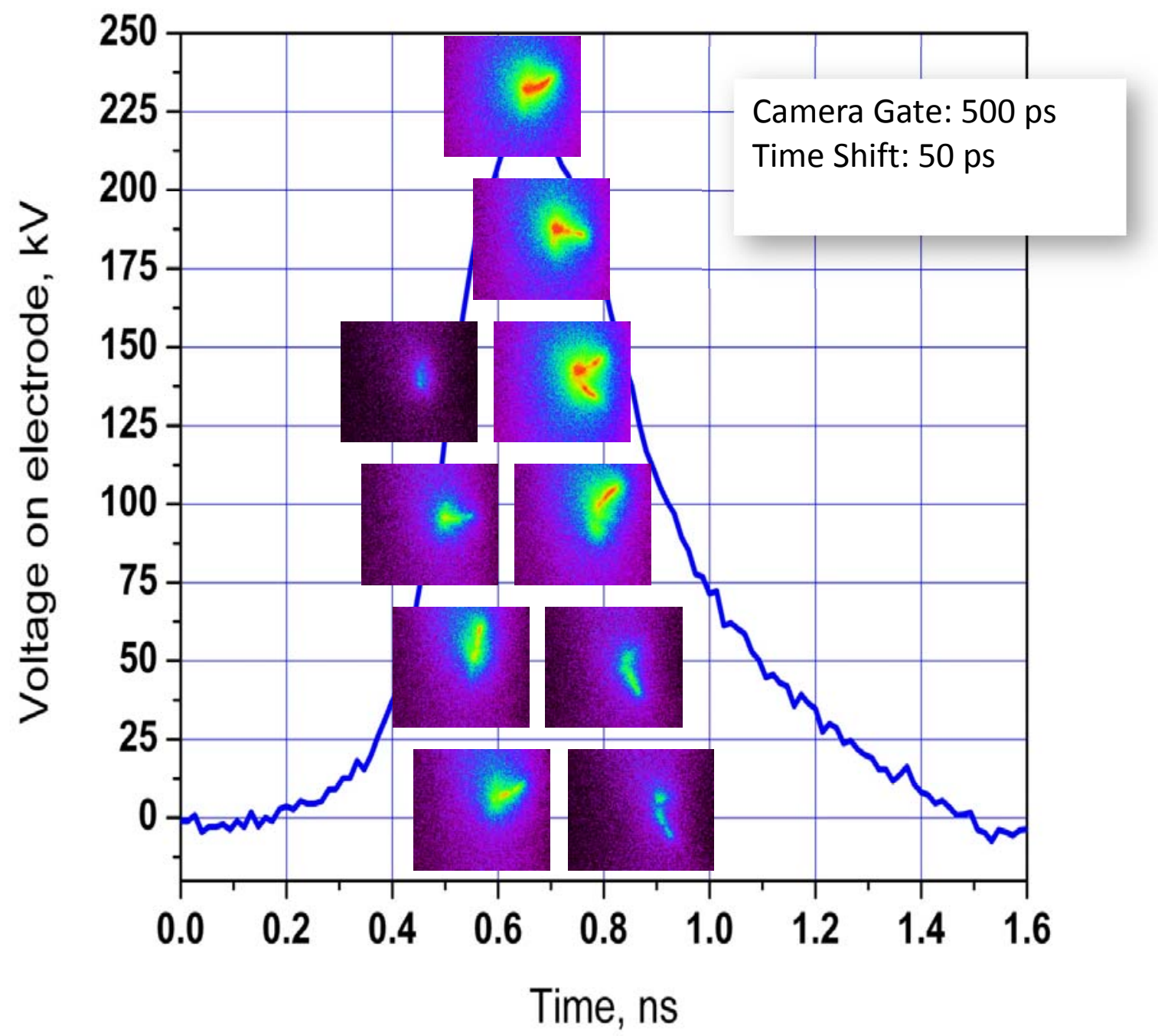

Figure 5 -26. Dynamics of discharge emission. Distilled water. $U=220 \mathrm{kV}$. dU/dt $=1.46$ $\mathrm{MV} / \mathrm{ns}$. Camera gate is $500 \mathrm{ps}$. Time shift between frames is $50 \mathrm{ps}$.

Observed propagation velocity reaches $5 \mathrm{~mm} / \mathrm{ns}(5,000 \mathrm{~km} / \mathrm{s} ; \sim 15 \% \boldsymbol{c})$ and is almost the same as a typical velocity of streamer propagation in air. Typical channel diameter is estimated as $d=50-100 \mu \mathrm{m}$. Thus the radial expansion velocity of the channel can be estimated as $v_{r}=r / \Delta t \approx 0.05 \mathrm{~mm} / 200 \mathrm{ps}=250 \mathrm{~km} / \mathrm{s}$ (Figure $5-27$ ). 

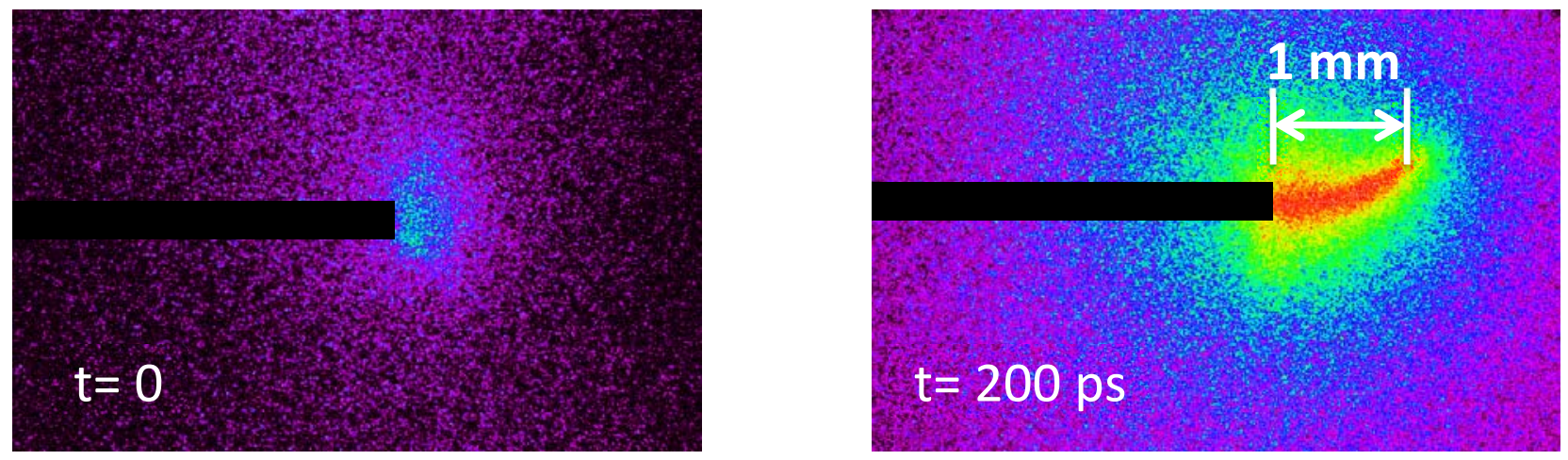

Figure $5-27$. Velocity of streamer propagation in water. $150 \mathrm{ps}$ rise time, $1.5 \mathrm{MV} / \mathrm{ns}$ : Growth rate $v_{z}=L / \Delta t \approx 1 \mathrm{~mm} / 200 \mathrm{ps}=5000 \mathrm{~km} / \mathrm{s} \sim 15 \% \boldsymbol{c}$. Expansion rate $v_{r}=r / \Delta t \approx 0.05 \mathrm{~mm} / 200 \mathrm{ps}$ $=250 \mathrm{~km} / \mathrm{s}$

\subsubsection{Ionization of the Liquid by E-Impact}

Next we will estimate the electrical field needed for water ionization using "dense gas" approach. We used cross-sections from the BOLSIG+ Solver (Hagelaar, 2008) to analyze the ratio between electron's attachment rate and the rate of ionization (Figure 5 -28).

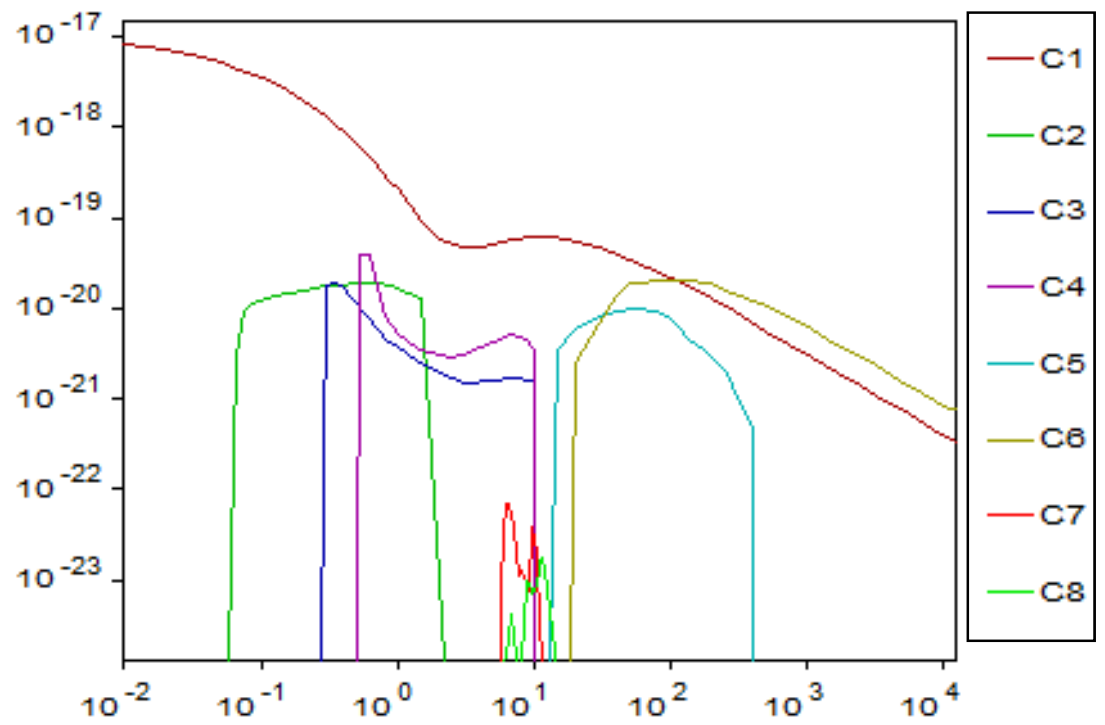

Figure 5 -28. Excitation cross-section (in $\left[\mathrm{m}^{2}\right]$ ) for $\mathrm{H}_{2} \mathrm{O}$ vapour (rarefied gas). $\mathrm{C}_{1}-$ elastic collisions; $\mathrm{C}_{2}$ - rotational excitation $(0.02 \mathrm{eV}) ; \mathrm{C}_{3}-$ vibrational excitation $(0.20 \mathrm{eV}) ; \mathrm{C}_{4}$ vibrational excitation $(0.45 \mathrm{eV}) ; \mathrm{C}_{5}-$ electronic excitation $(7.10 \mathrm{eV}) ; \mathrm{C}_{6}-$ ionization $(12.61 \mathrm{eV})$; $\mathrm{C}_{7}, \mathrm{C}_{8}$ - dissociative attachment. 


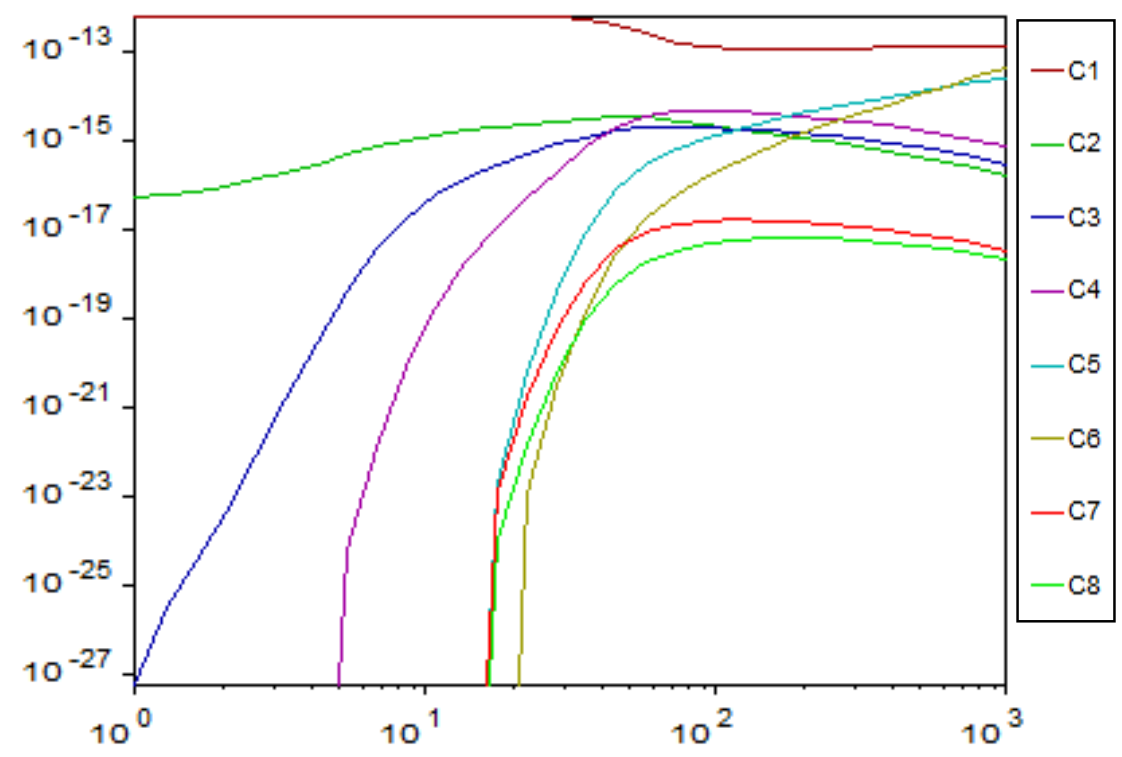

Figure 5 -29. Rate coefficients (in $\left[\mathrm{m}^{3} / \mathrm{s}\right]$ ) for different processes in water vapour in dependence on the $\mathrm{E} / \mathrm{n}$ value. $\mathrm{C}_{1}$ - transport collisions; $\mathrm{C}_{2}$ - rotational excitation; $\mathrm{C}_{3}, \mathrm{C}_{4}$ - vibrational excitation; $\mathrm{C}_{5}-$ electronic excitation; $\mathrm{C}_{6}-$ ionization; $\mathrm{C}_{7}, \mathrm{C}_{8}-$ dissociative attachment.

Figure 5 -29 demonstrates the rate coefficients for different processes in low-density water vapor. Again, it should be noted that these data cannot give quantitative description of the processes in liquid water and we will use them for evaluation purposes only. From Figure 5 -29 it is clearly seen that the ionization rate becomes higher than the attachment for $E / n>50 \mathrm{Td}$. In condense media multi-body attachments will play the major role. Taking into account a strong dependence of the rate constant of ionization from $E / n$ we can assume, nevertheless, that for $E / n$ $>100 \mathrm{Td}$ we can obtain fast ionization even in the condensed media. At normal conditions it means that the electric field value should be close or greater than $E_{c r i t}>30 \mathrm{MV} / \mathrm{cm}$. Almost the same estimation $(E \sim 10-20 \mathrm{MV} / \mathrm{cm})$ was obtained in the paper by Kolb et al. (2008).

Taking into account the geometrical parameters of the plasma channels (Figure 5 -27) we can estimate the radius of the streamer's tip as $r \sim 10 \mu \mathrm{m}$ and subsequent electric field as $E \sim 220 \mathrm{MV} / \mathrm{cm}(E / n \sim 670 \mathrm{Td})$. Thus the e-field near the discharge tip is an order of magnitude higher than the critical electric field estimated using dense-gas model. Thus we can assume that the direct ionization by electron impact takes place in the streamer head even in liquid water and it is possible to generate liquid non-equilibrium plasma in diffrent media.

\subsubsection{Chance of voids formation}

Water in laboratory conditions always contain trace impurities of dissolved gases. There are several different mechanisms of vapor bubbles (voids) formation in the liquid under discharge conditions (Bruggeman and Leys, 2009). There are: electrostatic mechanism, 
evaporation due to conductive current and Joule heat release, and initial bubbles expansion. In our experiments we were able to control the bubbles with a size above $5 \mu \mathrm{m}$ and did not find any trace of them both before and after the discharge. Below this limit (the limit was determined by spatial resolution of the photocathode and optical interference) everyone can assume the bubbles existence. Nevertheless the size of these initial bubbles should be very small in comparison with the plasma channel diameter $(\sim 100 \mu \mathrm{m})$.

Thus we can analyze the possibility of initial bubble expansion from possible "detectionlimited size" to "plasma channel size" during the discharge development time ( $100 \mathrm{ps})$. It is clear that to form the void in the liquid we need to generate a strong shock wave to accelerate the liquid from the axis of the discharge channel. Simple estimations give us the required flow velocities of about 100-200 km/s in radial direction. Could we achieve these parameters using low-energy discharge in water?

The analysis shows that the liquid motion with a velocity $30-35 \mathrm{~km} / \mathrm{s}$ (compression ratio 3.8) is possible only under nuclear-test explosion experiments (Trynin, 1994; Avrorin et al., 1980; Avrorin et al., 2006). One of the tests (Trynin, 1994) demonstrates shock wave propagation velocity $D=43.95 \mathrm{~km} / \mathrm{s}$, velocity of water behind shock wave front $U=32.54 \mathrm{~km} / \mathrm{s}$, compression ratio $s=r / r_{0}=3.852$ and pressure behind shock wave front $P=1430 \mathrm{GPa}$ at the distance of $2 \mathrm{~m}$ from the epicenter of the underground nuclear explosion. Total discharge energy in our experiments allow us to overheat the water in the discharge channel for $50 \mathrm{~K}$ only and definitely not enough to generate an ultra-strong shock wave in order to create the void. Thus pulsed discharges of sub-nanosecond range allows us to generate highly-ionized channels in condense media without voids formation. This approach creates numerous possibilities for "liquid plasma" applications like material synthesis, biophysical and biomedical plasma systems, light sources, and liquid-phase plasma-chemical systems. 


\section{Chapter 6 COOLING WATER TREATMENT USING PLASMA}

Water is used as a cooling medium in various industrial facilities such as large centralized air-conditioning systems and in thermoelectric power plants. In both cases, the cooling water plays an essential role in removing heat from condensers. According to the U.S. Geological Survey's (USGS) water use survey data (Feeley et al., 2008), thermoelectric generation accounted for 39\% (136 billion gallons per day (BGD)) of all freshwater withdrawals in the nation in 2000, second only to irrigation, see Figure 6-1 (Feeley et al., 2008). Furthermore, the average daily national freshwater consumption for thermoelectric power generation is predicted to increase from the current $4 \mathrm{BGD}$ for the production of approximately $720 \mathrm{GW}$ electricity to 8 BGD for $840 \mathrm{GW}$ in 2030, see Figure 6-2 (Feeley, 2008).

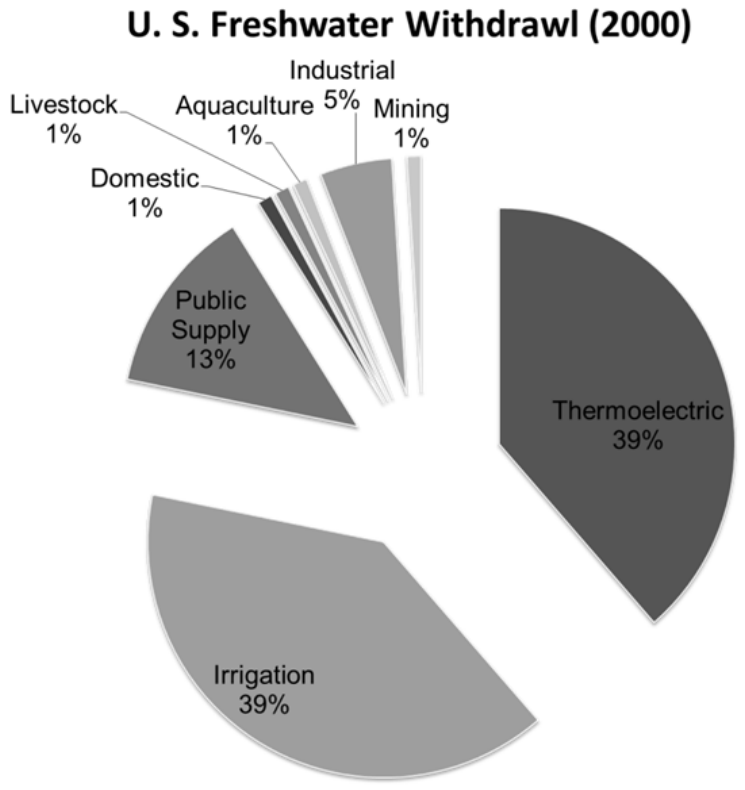

Figure 6-1. US freshwater withdrawal in 2000. Source: USGS, Estimated use of water in the United States in 2000, USGS Circular 1268, March 2004 (Add-up may not be equal to $100 \%$ due to rounding) 


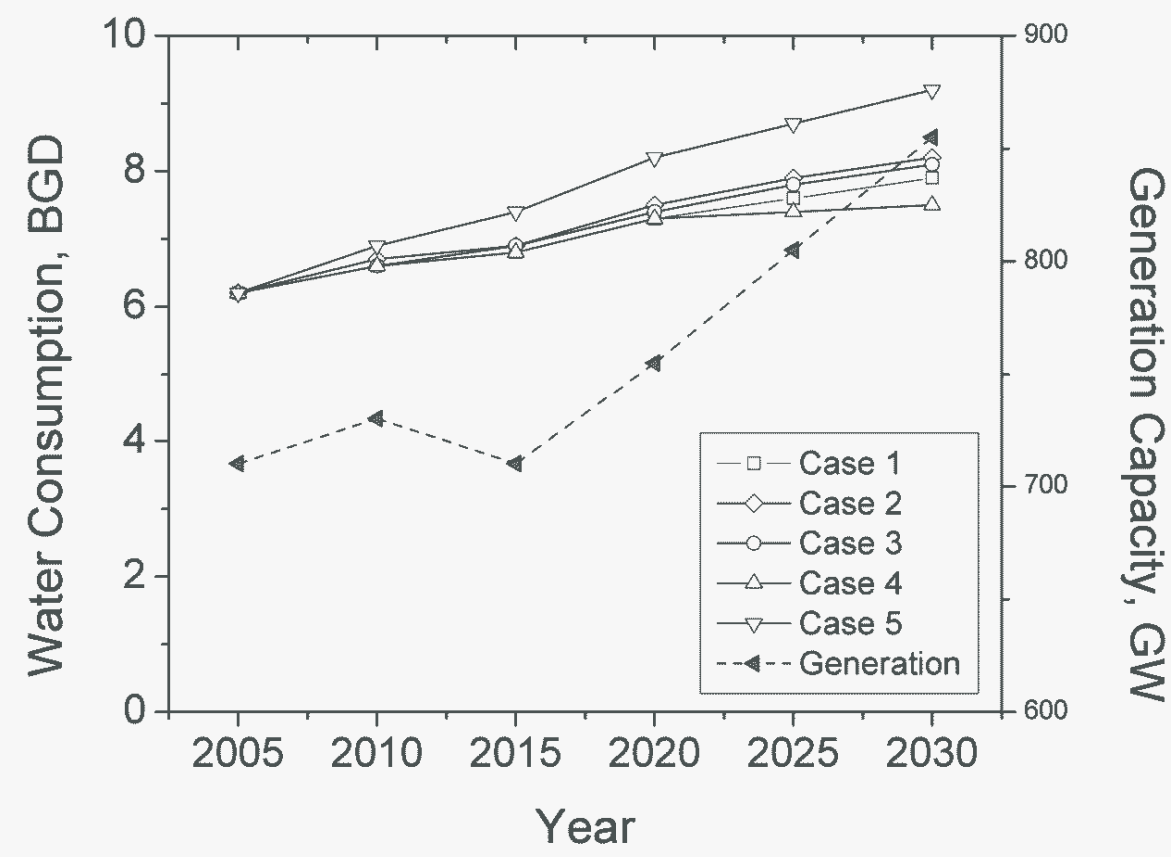

Figure 6-2. Average daily national freshwater consumption for thermoelectric power generation 2005-2030 (predicted). Source: DOE/Office of Fossil Energy's Energy \& Water R\&D Program. 2008 


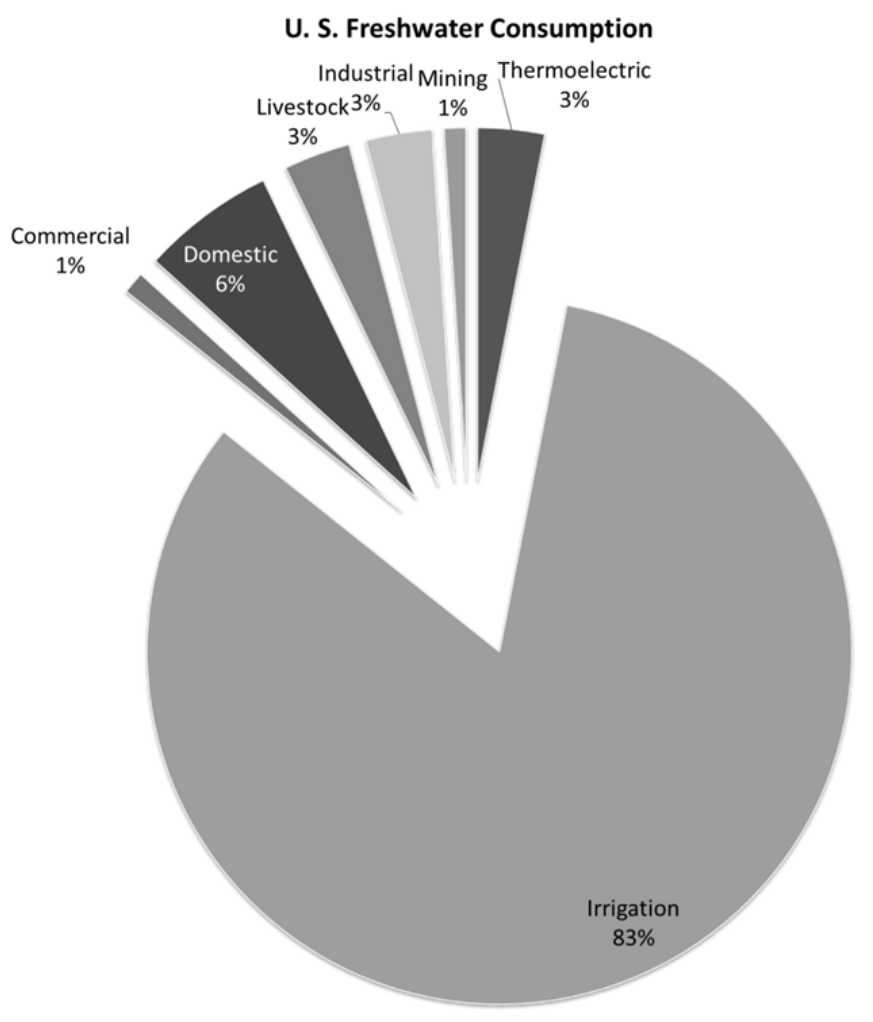

Figure 6-3. US freshwater consumption in 1995. Source: USGS, Estimated use of water in the United States in 1995, USGS Circular 1220, 1998 (Add-up may not be equal to $100 \%$ due to rounding)

In the cooling water management, it is important to distinguish between water withdrawal and water consumption. Water withdrawal represents the total water taken from a source, while water consumption represents the amount of water withdrawal that is not returned to the source. Freshwater consumption for the year 1995 (the most recent year for which these data are available) is presented in Figure 6-3. Freshwater consumption for thermoelectric uses appears low (only $3 \%$ ) when compared with other use categories (irrigation was responsible for $81 \%$ of water consumed). However, even at 3\% consumption, thermoelectric power plants consumed more than 4 BGD (Feeley et al., 2008).

A modern 1000-MW thermoelectric power plant with $40 \%$ efficiency would reject 1500 MW of heat at the full load. This is roughly equivalent to $512 \times 10^{6} \mathrm{Btu} / \mathrm{hr}$ and uses about $760,000 \mathrm{gal} / \mathrm{min}$ of circulating water based on $10{ }^{\circ} \mathrm{C}$ temperature difference in condenser (ElWakil, 1984). As heat is removed via the evaporation of pure water at cooling tower, the need for the makeup water is about $7500 \mathrm{gal} / \mathrm{min}$ for a typical fossil plant, which results in 10 million gallons a day (El-Wakil, 1984). 
One of the critical issues in the cooling water management is the condenser tube fouling by mineral ions such as calcium and magnesium. Since calcium carbonate $\mathrm{CaCO}_{3}$ is the most common issue in cooling water, one can use the word "calcium scale" to refer all scales caused by mineral ions. In order to prevent or minimize the condenser tube fouling, the COC in wet recirculation cooling systems is often kept at 3.5. Since increasing the cycles of concentration can reduce the amount of the makeup water, the water consumption can be reduced with the increased COC. For example, if one can increase the COC to 8, the freshwater consumption can be reduced by approximately $25 \%$, meaning that the makeup water can be reduced by 2.5 million gallons a day in a 1000-MW thermoelectric power plant.

Since the amounts of mineral ions in circulating cooling water primarily depend on the COC, the condenser tube fouling also depends on the COC. Hence, the issue in the cooling water management is to increase the $\mathrm{COC}$ without the condenser fouling problem. The present chapter deals with an innovative water treatment technology utilizing plasma discharges in water, with which one can increase the COC without the fouling problem in condenser tubes. The key issue is how to precipitate and remove mineral ions such as calcium and magnesium from circulating cooling water so that the calcium carbonate scales can be prevented at the condenser tubes and at the same time the COC can be increased.

In the next few sections, new developments of underwater plasma treatment at Drexel Plasma Institute for various applications will be discussed.

\subsection{Self-Cleaning Filtration Technology with Spark Discharge}

In modern wastewater treatment, filters are routinely used for removing unwanted particles from water. Conventionally, microfiltration methods are used to remove suspended particles from water. Whenever a filter is used in a water system, the pressure drop across the filter gradually increases with time and/or the flow rate gradually decreases with time. This reduced performance of a filter is due to the accumulation of impurities on the filter surface, and the clogged area becomes sites for bacteria growth for further reducing the opening in the filter surface, increasing the pumping cost. Therefore, in order to continuously remove suspended particles from water, the filter must be replaced frequently, a process which is prohibitively expensive in most industrial water applications. To overcome the drawbacks of frequent filter replacement, self-cleaning filters are commonly used in industry. Although there are a number of self-cleaning filter technologies available on the market, most self-cleaning filters use a complicated backwash method, which reverses the direction of flow during the cleaning phase. Furthermore, the water used in the backwash must be clean filtered water, which reduces the filter capacity. Aforementioned drawbacks of the conventional filter technologies motivated the authors to develop a new self-cleaning filter using spark-generated shockwaves.

As illustrated in previous chapters, strong shockwaves can be formed during the process of pulsed arc or spark discharge. The energy transferred to the acoustic energy can be calculated as (Lu et al., 2002): 


$$
E_{\text {acoustic }}=\frac{4 \pi r^{2}}{\rho_{0} C_{0}} \int\left(P(r, t)-P_{0}\right) d t
$$

where $r$ is the distance from the spark source to the pressure transducer, $\rho_{0}$ is the density of water, $\mathrm{C}_{0}$ is the speed of sound in water, $\mathrm{P}_{0}$ is the ambient pressure. One can conclude that the pressure created by the spark discharge is much higher than the ambient pressure at positions close to the source. Traditionally, the high-pressure shockwave is studied for high-voltage insulation and rock fragmentation, while recently it has found more applications in other areas including extracorporeal lithotripsy and metal recovery from slag waste (Bluhm et al., 2000, Akiyama et al., 2007, Wilson et al., 2006).

In order to validate the concept of using spark discharge for filter cleaning, an experimental setup was built where discharges could be produced in water, and pressure drop across a filter surface was measured over time at various spark frequencies and flow conditions. It was hypothesized that the energy deposited by the spark shockwave onto water-filter interface was enough to remove the contaminants having Van der Waals bonds with filter surface. The objective of the study was to examine the feasibility of a self-cleaning water filtration concept using spark discharges in water.

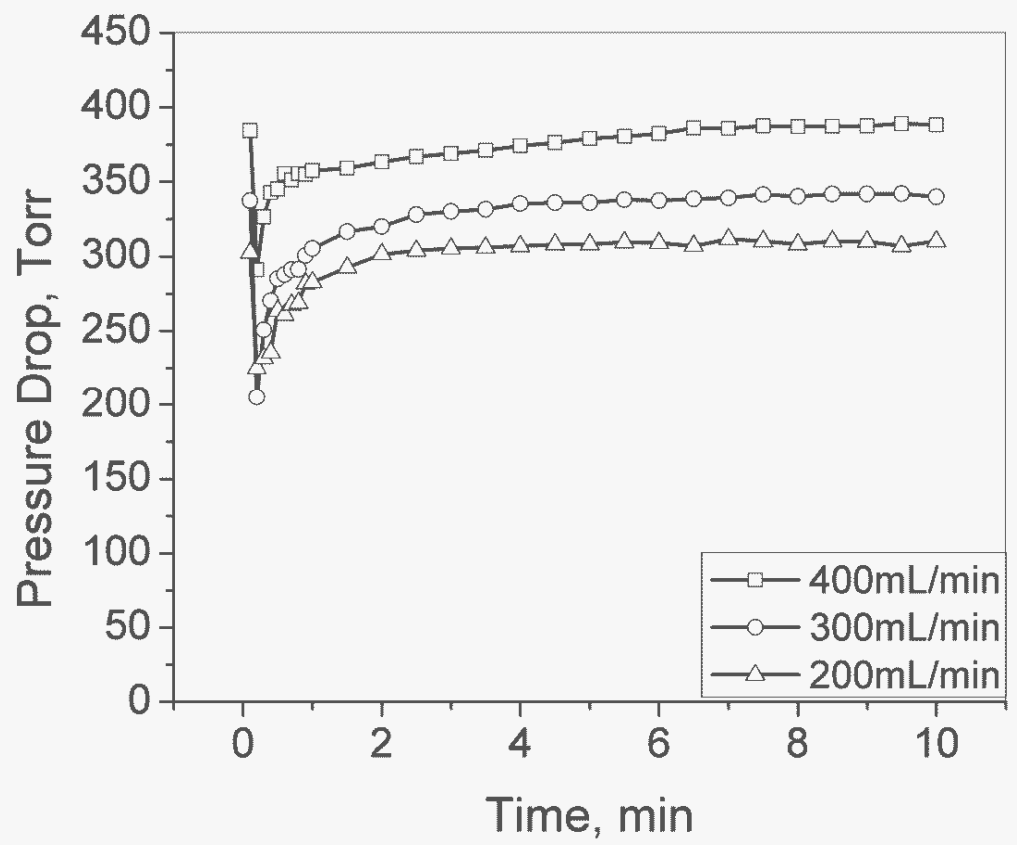

Figure 6-4. Variations of pressure drop after one single spark discharge at three different flow rates with an artificially hardened water (Yang et al., 2009).

Figure 6-4 shows the long-time response of the pressure drop across the filter surface after one single spark discharge at different flow rates. One could visually observe that some particles 
were dislodged from the filter surface and were pushed away from the filter surface by tangential flow, and a sudden change in the pressure drop immediately following the single spark discharge confirmed the removal of the deposits from the filter surface.

The cleaning effect can be explained by the pressure pulse produced by spark discharge. A number of researchers studied the bubble growth by spark discharge in water (Joseph and Miksis, 1980; Inoue and Kobayashi, 1993; Prosperetti and Lezzi, 1986; Lezzi and Prosperetti, 1987). One of the most effective models is Kirkwood-Bethe model as given below (Lu et al., 2002):

$$
\left(1-\frac{\dot{R}}{C}\right) R \ddot{R}+\frac{3}{2}\left(1-\frac{\dot{R}}{3 C}\right) \dot{R}^{2}=\left(1+\frac{\dot{R}}{C}\right) H+\left(1-\frac{\dot{R}}{C}\right) \frac{R}{C} \dot{H}
$$

where $\mathrm{C}$ and $\mathrm{H}$ are the speed of sound of the water and the specific enthalpy at the bubble wall, respectively. $\mathrm{R}$ is the radius of the bubble wall. The overdots denote the derivatives with respect to time. By expressing the time derivative of specific enthalpy as a function of derivative of plasma pressure P inside the bubble, Lu showed that it was possible to solve P as ( $\mathrm{Lu}$ et al., 2002):

$$
P\left(r, t_{r}\right)=A\left[\frac{2}{n+1}+\frac{n-1}{n+1}\left(1+\frac{n+1}{r C^{2}} G\right)^{1 / 2}\right]^{(2 n /(n-1))}-B
$$

where $\mathrm{A}, \mathrm{B}$ and $\mathrm{n}$ are constants $(\mathrm{A}=305.0 \mathrm{MPa}, \mathrm{B}=304.9 \mathrm{MPa}, \mathrm{n}=7.15), \mathrm{r}$ is the distance from the source of the spark to the pressure transducer, and

$$
G=R(H+\dot{R} \dot{R} / 2), t_{r}=t+(r-R) / C_{0}
$$

Using the above equation, Lu et al. simulated that for a spark discharge with energy of 4.1 $\mathrm{J} /$ pulse, the maximum pressure at a distance of $0.3 \mathrm{~m}$ could be up to $7 \mathrm{~atm}$ ( $\mathrm{Lu}$ et al., 2002). With a single pulse, it took approximately $3 \mathrm{~min}$ for the pressure drop to return to its asymptotic value after the application of the single spark discharge. This suggests that one needs to repeatedly apply spark discharges to effectively remove the particles from the filter surface over an extended period. 


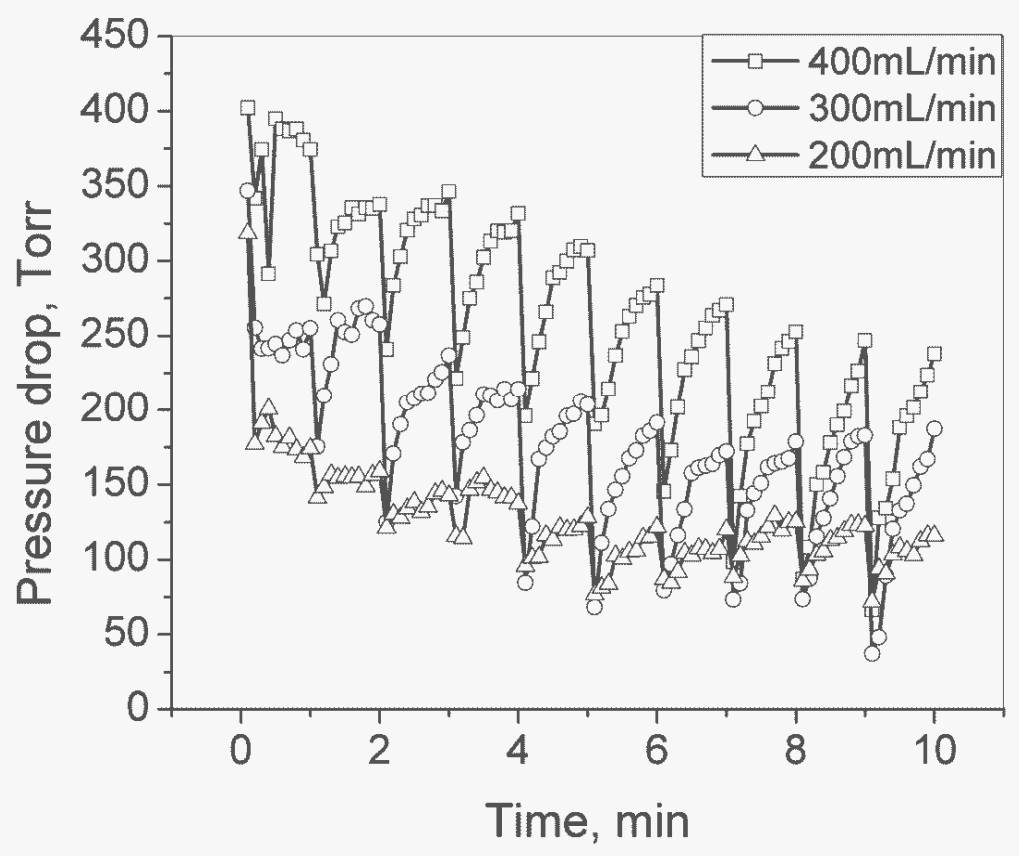

Figure 6-5. Changes in pressure drop under repeated pulsed spark discharges with an artificially hardened water (Yang et al., 2009).

Figure 6-5 shows the changes in the pressure drop over time for three different flow rates. One spark discharge was applied every minute from the supply-water side (i.e., untreated water side) where the accumulation of suspended particles took place. For the case of $300 \mathrm{~mL} / \mathrm{min}$, the pressure drop decreased from the maximum asymptotic value of 350 Torr to 230 Torr after the first spark discharge. Since water with particles was continuously circulated through the filter surface, the pressure drop began to increase immediately after the completion of the first spark discharge as shown in Figure 6-4. The second and third spark discharges further reduced the pressure drop to 170 and 125 Torr, respectively. The pressure drop again began to increase immediately after each spark discharge. The sixth spark discharge brought the pressure drop down to a value of approximately 65 Torr, and subsequent spark discharges almost resulted in the minimum value of the pressure drop. For the cases of 200 and $400 \mathrm{~mL} / \mathrm{min}$, similar trends of the changes in the pressure drop were observed. 


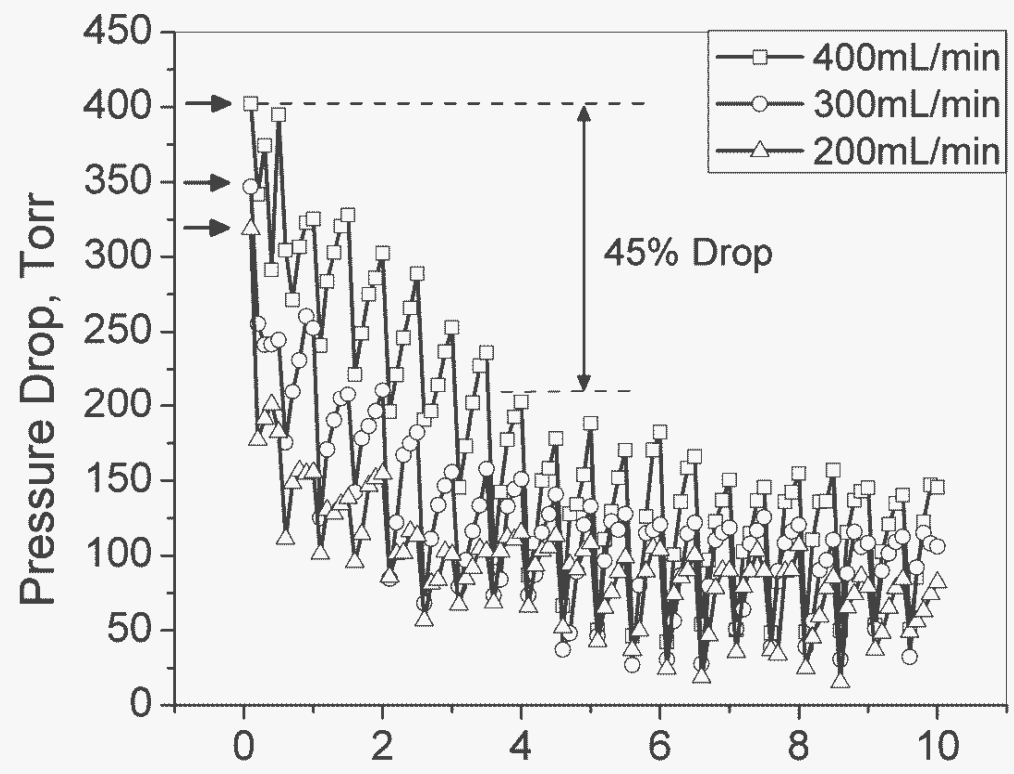

(a)

Time, $\min$

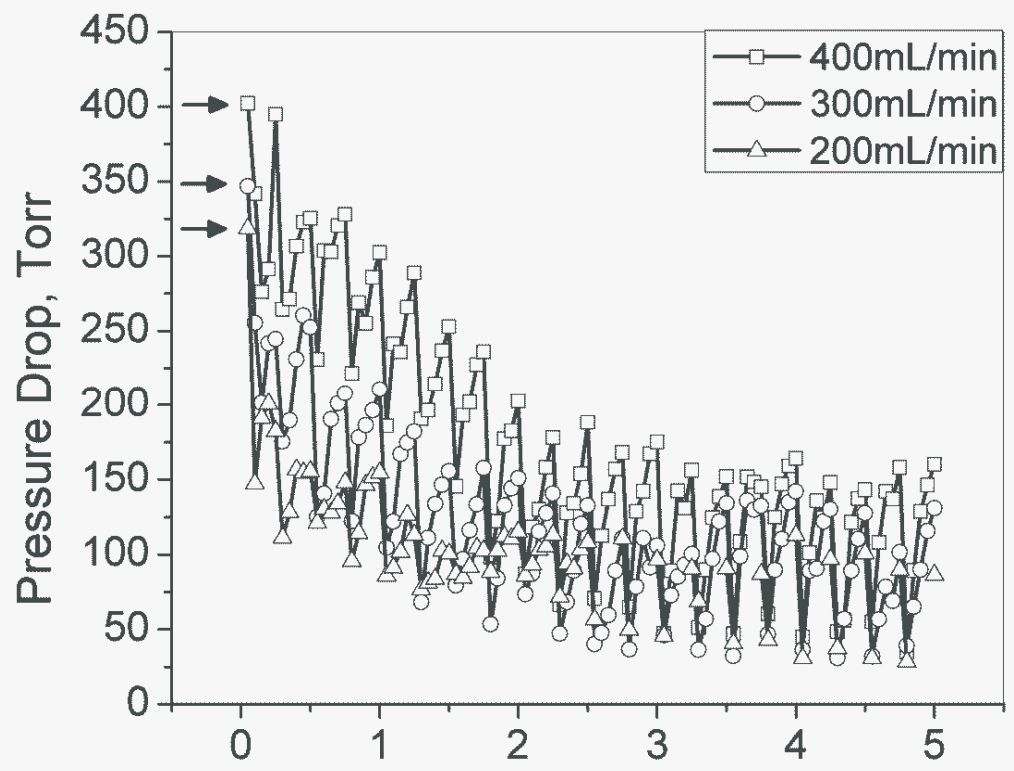

(b)

Time, $\min$

Figure 6-6. Changes in pressure drop under repeated pulsed spark discharges with frequencies of (a) 2 pulses/min and (b) 4 pulses/min (Yang et al., 2009). 
Figures 6(a) and 6(b) show the changes in the pressure drop under repeated pulsed spark discharges with frequencies of 2 and 4 pulses/min, respectively. Three horizontal arrows indicate the original asymptotic values for three different flow rates, which were the maximum pressure drop due to clogged filter surface by calcium carbonate deposits. First spark discharge significantly reduced the pressure drop in both cases. After that, the rate of the reduction slowed down. The pressure drop oscillations observed after the application of spark pulses reached quasi-steady conditions after about 10 pulses for both cases. In these oscillations, the maximum pressure drop decreased to about $45 \%$ of its original asymptotic value, while the minimum pressure drop was close to that of the clean filter. These results demonstrate the validity of the present spark discharge method. Note that the present cleaning method using the spark discharge does not require a back wash to remove deposits from the filter surface nor stopping the flow. Furthermore, the present spark discharge method can maintain the pressure drop across the filter at a rather low value (i.e., almost close to the initial clean state), thus providing a means to save not only fresh water but also electrical energy both for the operation of pump and for the back wash in the conventional back-wash system.

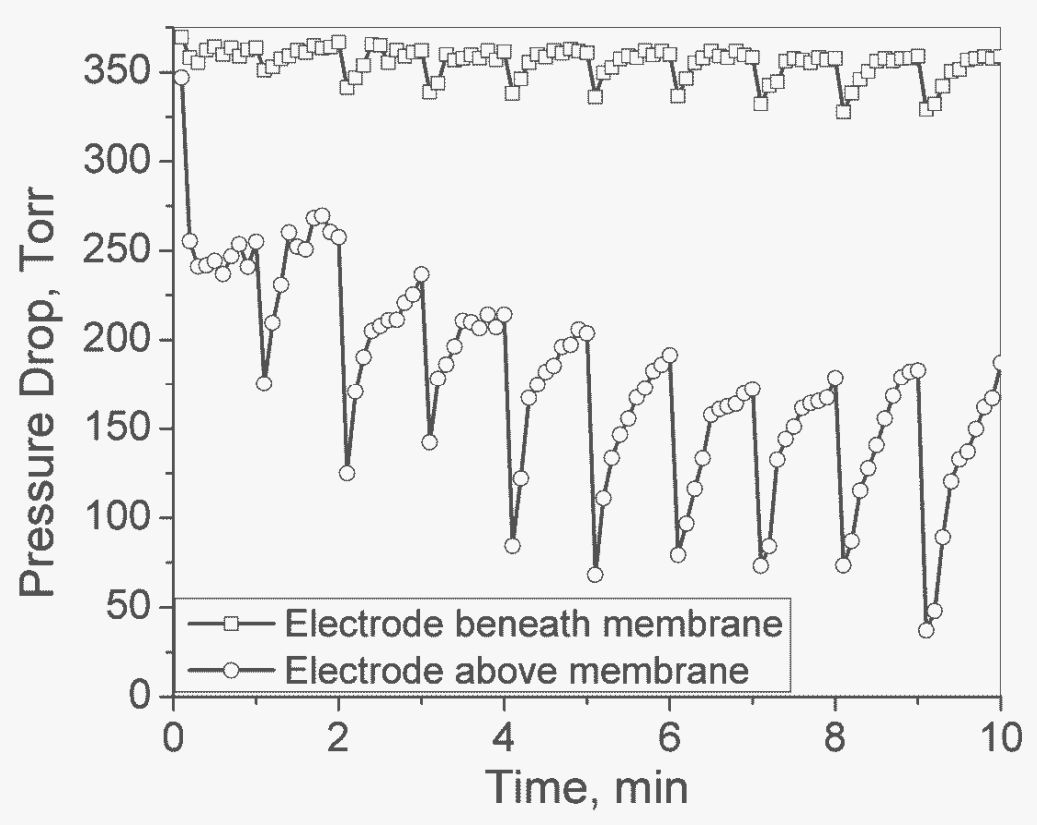

Figure 6-7. Comparison of pressure drop across filter membrane under repeated pulsed spark discharges in water: electrode beneath membrane vs. electrode above membrane (Yang et al., 2009).

Figure 6-7 shows the changes in the pressure drop over time with the anode electrode placed beneath the filter membrane (i.e. plasma discharge was applied from the treated water side). In this case, the momentum transfer from the shockwave to particles on the filter surface 
was indirect and had to go through the membrane. Figure 6-7 clearly shows that the pressure drop did not improve significantly in this case, indicating that the cleaning effect was negligible comparing with the case when the electrode was placed at the untreated water side. The fact that the momentum transfer from the shockwave to the membrane was weak is actually good news. The low energy transfer rate means that the present spark discharge may not deform the membrane significantly and therefore will not damage the membrane, and has the potential to be applied in the cleaning of more delicate membranes such as in a reverse osmosis as well as porous filters over an extended period of time.

\subsection{Calcium Carbonate Precipitation with Spark Discharge}

As mentioned in the introduction section, thermoelectric generation accounted for $39 \%$ (136 billion gallons per day) of all freshwater withdrawals in U. S. in 2000, second only to irrigation (Feeley et al., 2008). Each kilowatt-hour ( $\mathrm{kWh}$ ) of thermoelectric generation requires the withdrawal of approximately 25 gallons of water, which is primarily consumed for cooling purposes.

Since heat removal from condenser tubes requires the evaporation of pure water, the concentration of the mineral ions such as calcium and magnesium in the circulating cooling water increases with time. Even though the makeup water is relatively soft, the continuous circulation eventually increases the hardness of the water due to pure water evaporation. These mineral ions, when transported through piping in ordinary plumbing system, can cause various problems, including the loss of heat transfer efficiencies in condensers and pipe clogging due to scale formation (Panchal and Knudsen, 1998; Somerscales, 1990). Thus, in order to maintain a certain calcium hardness level in the cooling water, one must discharge a fraction of water through blowdown and replace it with makeup water.

In a typical cooling tower application, the cycle of concentration (COC) in cooling water is often maintained at 3.5. That means if the calcium carbonate hardness of the makeup water is $100 \mathrm{mg} / \mathrm{L}$, the hardness in the circulating cooling water is maintained at approximately 350 $\mathrm{mg} / \mathrm{L}$. If the COC can be increased through continuous precipitation and removal of calcium ions, one can significantly reduce the amounts of makeup and blowdown water, resulting in the conservation of fresh water.

Various chemical and non-chemical methods are used to prevent scaling and thus increase the COC. Among them the scale-inhibiting chemicals like chlorine and brominated compounds were the best choice for the control of mineral fouling. Although it had a high success rate, there were also many disadvantages and concerns in their use. Aside from the high cost of chemicals, more stringent environmental laws increased the costs associated with their storage, handling and disposal. These chemicals also pose danger on human health and environment with accidental spills, or accumulated chemical residues over a long period of time (Panchal and Knudsen, 1998; Muller-Steinhagen, 2000). Thus, there is a need for new approach which is safe and economical from both environmental and cost points of view in cleaning and maintenance of heat exchangers. Physical water treatment (PWT) is a non-chemical method to mitigate 
mineral fouling with the use of electric or magnetic fields, catalytic surfaces, ultrasounds, or sudden pressure changes. Numerous studies have been reported for the effectiveness of ultrasound (Radler and Ousko-Oberhoffer, 2005), solenoid coils (Cho et al., 2004; Cho et al., 2003; Cho et al., 2005), magnetic fields (Fathi et al., 2006; Xiaokai 2008), catalytic material (Coetzee et al., 1998; Lee et al., 2006), and electrolysis (Gabrielli et al., 2006).

Herein the pulsed-spark-discharge-assisted precipitation of dissolved calcium ions in hard water system is presented. First, the chemistry behind the $\mathrm{CaCO}_{3}$ precipitation is described. The main reactions of precipitation and dissolution of calcium carbonate in hard water system are (Snoeyink and Jenkins, 1982):

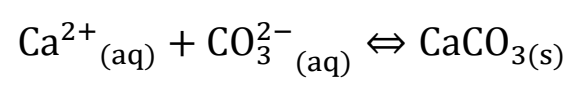

and

$$
\mathrm{CaCO}_{3(\mathrm{~s})}+\mathrm{H}^{+}{ }_{(\mathrm{aq})} \Leftrightarrow \mathrm{Ca}^{2+}{ }_{(\mathrm{aq})}+\mathrm{HCO}_{3(\mathrm{aq})}^{-}
$$

In a saturated condition, the forward reaction of the precipitation of $\mathrm{CaCO}_{3}$ does not take place as both calcium and bicarbonate ions are hydrated. When water is supersaturated and there is sufficient energy supplied, the water molecules are disturbed or become freed from the ions, resulting in the precipitation of $\mathrm{CaCO}_{3}$. Equation (6-6) shows the dissolution of solid calcium carbonate by acid, a process that takes place during acid cleaning of scaled heat exchangers. Generally, one needs to concern with the above two reactions. In reality, precipitation and dissociation reactions are much more complicated. The rate of calcium and carbonate ions recombination and crystallization is controlled by three reactions. Reaction 1 relates the dissociation of bicarbonate ions into the hydroxyl ions $\mathrm{OH}^{-}$and carbon dioxide (MullerSteinhagen, 1999):

$$
\mathrm{HCO}_{3(\mathrm{aq})}^{-} \Leftrightarrow \mathrm{OH}_{(\mathrm{aq})}^{-}+\mathrm{CO}_{2(\mathrm{aq})} \quad(\text { Reaction } 1)
$$

The forward reaction indicates the dissociation of the bicarbonate ions, which is the most critical step from the precipitation process. Of note is that the bicarbonate ions do not cause any harm in terms of scaling as long as they remain as bicarbonate ions. Reaction 1 shows the first step in the conversion of bicarbonate to carbonate ions. The presence of hydroxide ions is best indicated by a local increase in $\mathrm{pH}$, and carbon dioxide typically evolves from the water as gas over time.

In Reaction 2 hydroxyl ions produced from Reaction 1 further react with existing bicarbonate ions, producing carbonate ions and water (Muller-Steinhagen, 1999):

$$
\mathrm{HCO}_{3(\mathrm{aq})}^{-}+\mathrm{OH}_{(\mathrm{aq})}^{-} \Leftrightarrow \mathrm{H}_{2} \mathrm{O}_{(\mathrm{l})}+\mathrm{CO}_{3(\mathrm{aq})}^{2-} \quad \text { (Reaction 2) }
$$


Reaction 3 is the reaction between calcium and carbonate ions, resulting in the precipitation and crystallization of calcium carbonate particles (Muller-Steinhagen, 1999):

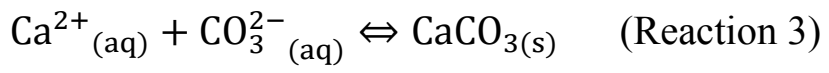

Table 6-1. Thermochemical data of Reactions 1, 2 and 3 (Snoeyink and Jenkins, 1982)

\begin{tabular}{|l|l|l|l|}
\hline & Reaction 1 & Reaction 2 & Reaction 3 \\
\hline$\Delta \mathrm{H}(\mathrm{kJ} / \mathrm{mol})$ & 48.26 & -41.06 & 12.36 \\
\hline$\Delta \mathrm{G}(\mathrm{kJ} / \mathrm{mol})$ & 43.60 & -20.9 & -47.70 \\
\hline
\end{tabular}

Table 6-1 presents the thermochemistry of the above three reactions (Snoeyink and Jenkins, 1982). The values of $\Delta \mathrm{H}$ and $\Delta \mathrm{G}$ give some useful insights into the behavior of the system of reactions. The endothermic Reaction 1 is the most rate-limiting, since it needs a relatively large input of energy to continue in the forward direction based on the high enthalpy value. The Gibbs free energy is relatively high, thus this reaction will tend to form bicarbonate ions, unless this energy restriction is overcome. In order for hydroxide ions to be produced as a result of dissociation of the bicarbonate ions, the energy required for the reaction can be calculated as:

$$
\frac{48,260 \mathrm{~J}}{\mathrm{~mol}} \frac{1 \mathrm{~mol}}{6.02 \times 10^{23} \mathrm{ions}}=0.801 \times 10^{-19} \mathrm{~J}(\text { or } \sim 0.5 \mathrm{eV}) \quad(6-10)
$$

To overcome the barrier, enough energy needs to be added to water so that the bicarbonate ions can be dissociated, and subsequently precipitating dissolved calcium ions to $\mathrm{CaCO}_{3}$ via Reactions 2 and 3.

Thus, it is clear that dissociation of bicarbonate ions plays an important role in the precipitation process, as observed in the experiments. From thermodynamics, the reaction rate coefficient $\mathrm{k}$ for Reaction 1 is:

$$
\mathrm{k}=\mathrm{Ae}^{-\mathrm{E}_{\mathrm{a}} / \mathrm{T}} \quad(6-11)
$$

where $\mathrm{A}$ is a constant, $\mathrm{E}_{\mathrm{a}}$ is activation energy for the reaction, $\mathrm{T}$ is the system temperature (in the unit of eV). It is obvious that higher temperature will lead to higher precipitation rate. For example, when hard water is heated (i.e., volume heating) by boiling or microwave, the calcium carbonate deposit can be usually observed in bulk solution or heated surface.

For plasma treatment cases, the total input energy was on the level on $\mathrm{kJ} / \mathrm{L}$, so that no significant volume heating exists. However, direct pulsed spark discharges in water have been shown to generate a temperature up to $5,000-10,000 \mathrm{~K}$ (about $0.5-1 \mathrm{eV}$ ) inside the plasma channel (Sunka et al., 1999), a phenomenon which is sufficient to induce direct pyrolysis of 
bicarbonate ions. The high temperature zone is highly localized around the plasma channel, so that higher efficiency could be achieved over volume heating under the same energy input level due to an exponential dependence of the reaction rate on the temperature. The maximum temperature depends on both plasma power and water properties.

Furthermore, the thermal dissociation of bicarbonate ions may be enhanced by the emission of UV light from the high-temperature plasma channel, which functions as a blackbody radiation source. Full-spectrum UV radiation was found to be produced from a spark discharge (Sunka, 2001). The VUV ( $\lambda=75-185 \mathrm{~nm})$ emitted would be absorbed by the water layer immediately surrounding the plasma channel, leading to the expansion of the hightemperature zone (Locke et al., 2006). However, the amount of solution that can be treated directly by the thermal process is still limited by the small volume of the high-temperature zone. Another factor that may contribute to the dissociation of bicarbonate ions is the electric field. There are reports about the increase in the nucleation rate of different crystals when subjected to external electric fields (He and Hopke, 1993; Chibowski et al., 1994; Katz et al., 1994). Dhanasekaran and Ramasamy (Dhanasekaran and Ramasamy, 1986) studied the free energy required for the formation of a two-dimensional nucleus of any possible shape under electric field. They calculated the critical free energy for nucleation as

$$
\Delta \mathrm{G}=\frac{\beta^{2} \sigma^{2}}{4 \mathrm{kT}\left(\ln \alpha+\varphi \mathrm{E}^{2}\right)}
$$

where $\beta$ is a constant depending on the geometrical shape of the nucleus, $\sigma$ is the interfacial tension, $\mathrm{k}$ is the Boltzmann constant, $\mathrm{T}$ is the temperature, $\alpha$ is the supersaturation ratio, $\mathrm{E}$ is the external electric field, and $\varphi$ is defined as $-\varepsilon_{0} \mathrm{v}\left[\left(\frac{1}{\varepsilon_{\mathrm{s}}}-\frac{1}{\varepsilon_{1}}\right) \sin ^{2} \theta+\left(\varepsilon_{\mathrm{s}}-\varepsilon_{1}\right) \cos ^{2} \theta\right] / 8 \pi \mathrm{kT}$, where $\mathrm{v}$ is the volume of the crystalline nucleus, $\theta$ is the angle between the direction of the electric field and the nucleation surface, and $\varepsilon_{\mathrm{s}}$ and $\varepsilon_{1}$ are the dielectric constants of the nucleus and solution, respectively. According to Dhanasekaran's theory, the free energy for nucleation decreases as the strength of electric field increases at certain angles, which leads to a higher nucleation rate.

The effect of electric field could also be explained by the disruption of the electric double layer of hydrated ions. Dissolved calcium and bicarbonate ions do not react at room temperature as both ions are surrounded by water molecules forming electric double layers. A number of researchers postulated that the magnetic or electric field, if strong enough, might disrupt the electric double layer and initiate the precipitation (Gehr et al., 1995; Baker and Judd, 1996; Higashitani and Oshitan, 1997). In order for an electric field to directly affect the electric double layer near a negatively charged surface, one needs an electric field that provides force which is able to overcome the force in the electric double layer. Typically, there are approximately two electron volts across an electric double layer, and the Debye length for a dilute solution like water is about $10 \mathrm{~nm}$ (Levine, 1978; Probstein, 1989). Thus, the electric field 
required to directly affect the electric double layer becomes approximately $2 \mathrm{~V} / 10 \mathrm{~nm}=2 \times 10^{8}$ $\mathrm{V} / \mathrm{m}$. To initiate pulsed electric discharges in water using a point-to-plane electrode system, it is necessary to have a high intensity electric field at the tip of the point electrode, which can be calculated as

$$
\mathrm{E} \approx \frac{\mathrm{V}}{\mathrm{r}_{\mathrm{e}}}
$$

where $\mathrm{V}$ is the applied voltage, and $\mathrm{r}_{\mathrm{e}}$ is the radius of curvature at the tip of the electrode. For the present study, $\mathrm{V}=24 \mathrm{kV}$ and $\mathrm{r}_{\mathrm{e}} \approx 200 \mu \mathrm{m}$, leading to an electric field of $1.2 \times 10^{8} \mathrm{~V} / \mathrm{m}$, which is comparable to the electric field required to disrupt the hydration shells of the ions, and could possibly accelerate the dehydration process of ionic pair association. Additionally, unlike the highly localized thermal effect, the electric effect is not limited to the vicinity of the electrode tip. After the initiation of pulsed discharge, multichannel streamers with lengths up to several centimeters could be produced when propagating from one electrode to the other electrode. An and his coworkers (An et al., 2007) measured the radius of the streamers and inferred that the electric field at the tip of the self-propagating streamers was more than $2 \times 10^{9}$ $\mathrm{V} / \mathrm{m}$. Subsequently, the bicarbonate ions may be dissociated along the propagation path of the streamers leading to the precipitation of calcium carbonate in bulk water as described earlier.

\subsubsection{Effect of Plasma on Cooling Water}

Figure 6-8 presents the variation of $\mathrm{pH}$ and calcium ion concentration as a function of time and input plasma energy, and their comparisons with no-treatment cases. For the case of Sample 1, the calcium ion concentration dropped from the initial value of $96 \mathrm{mg} / \mathrm{L}$ to $71 \mathrm{mg} / \mathrm{L}$ after 10 min of plasma treatment. After that the hardness would reach an asymptotic value and not decrease with further input of energy. Accompanying the drop of the calcium ion concentration, the $\mathrm{pH}$ of the treated water sample decreased from 8.67 to 8.51 , possibly because of the liberation of $\mathrm{H}^{+}$ions according to the following reaction:

$$
\mathrm{Ca}^{2+}+\mathrm{HCO}_{3}^{-} \rightarrow \mathrm{CaCO}_{3}+\mathrm{H}^{+} \quad(6-14)
$$

Additionally, the ionization of water molecules by high-energy electrons from plasma discharge may also contribute to the decrease of the $\mathrm{pH}$ through the following reaction:

$$
\begin{aligned}
& \mathrm{H}_{2} \mathrm{O}+\mathrm{e} \rightarrow \mathrm{H}_{2} \mathrm{O}^{+}+2 \mathrm{e} \\
& \mathrm{H}_{2} \mathrm{O}^{+}+\mathrm{H}_{2} \mathrm{O} \rightarrow \mathrm{H}_{3} \mathrm{O}^{+}+\mathrm{OH}^{*}
\end{aligned}
$$



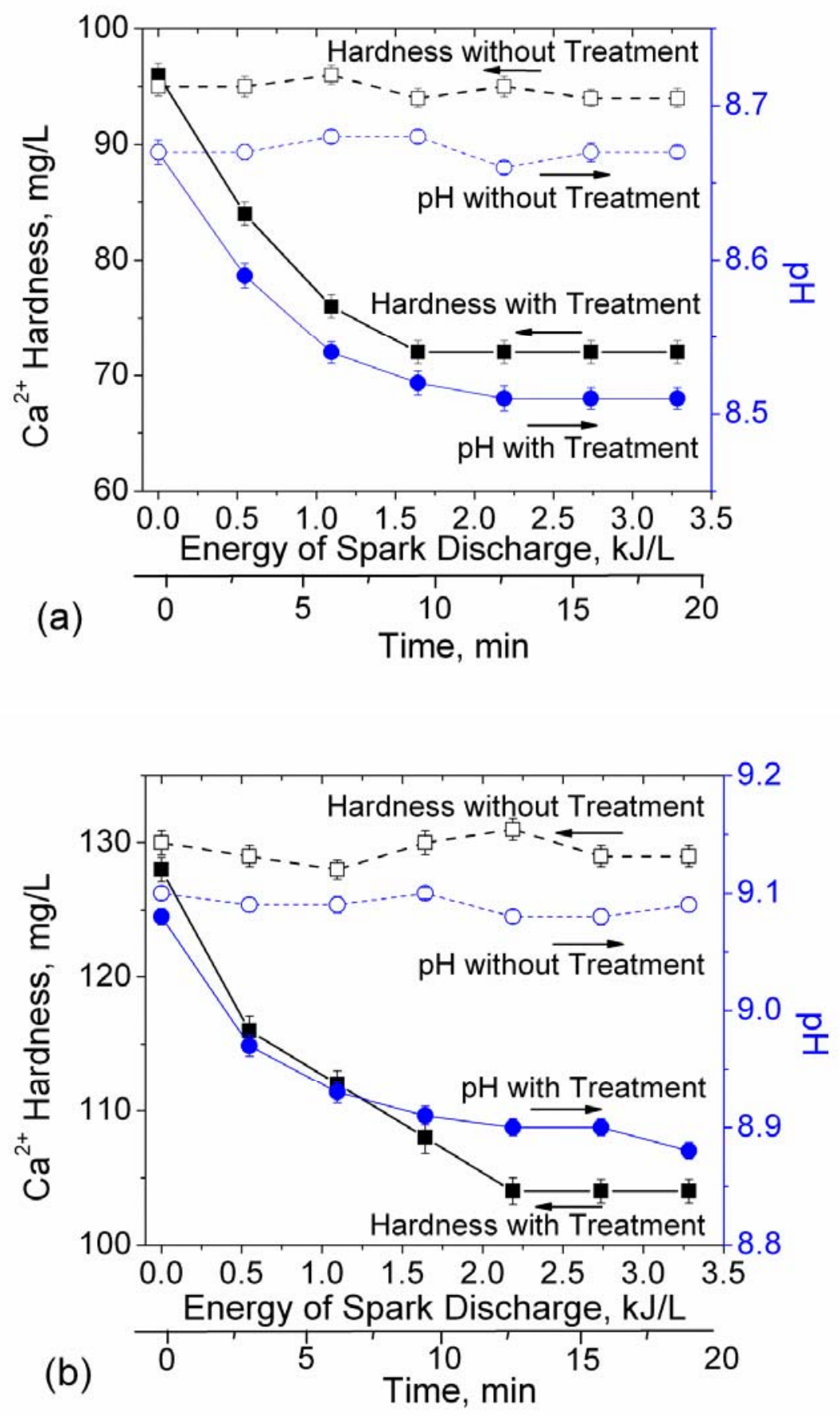


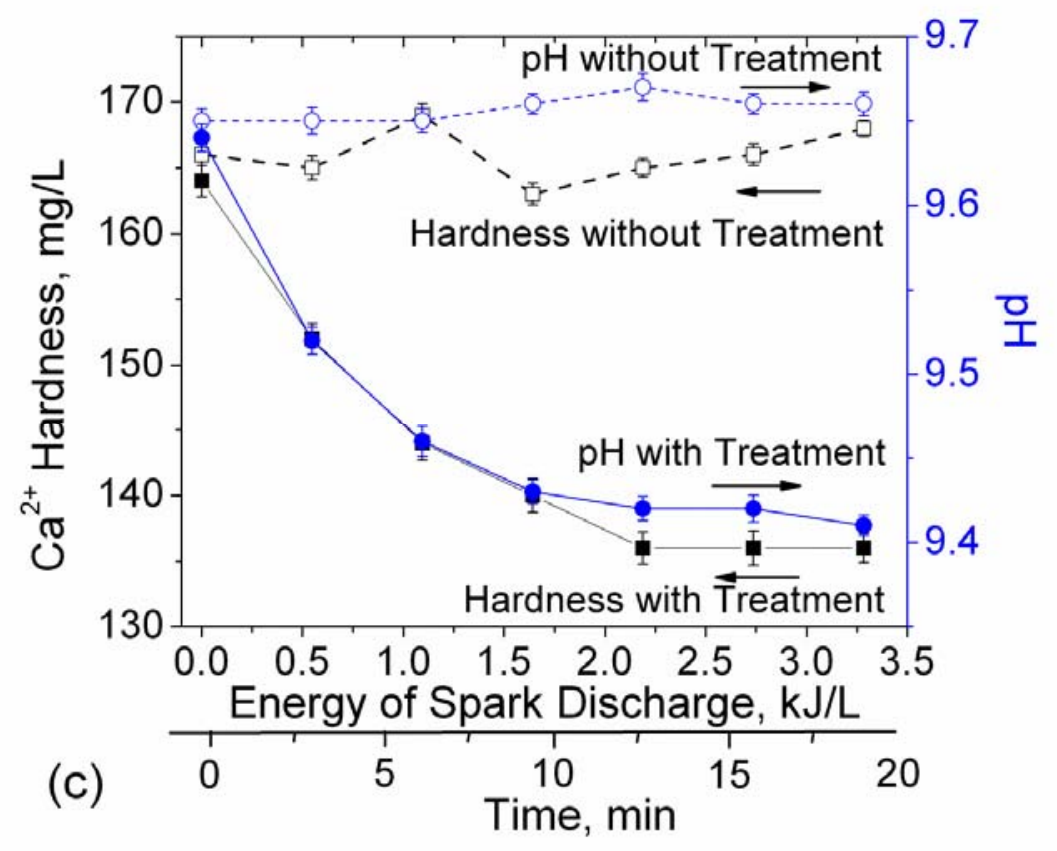

Figure 6-8. Variations of $\mathrm{pH}$ and $\mathrm{Ca}^{2+}$ hardness over time with and without plasma treatment: (a) Sample 1; (b) Sample 2; (c) Sample 3. See Table 6-2 for more information on three samples. (Yang et al., 2010a).

Samples 2 and 3, with initial calcium ion concentration of 128 and $164 \mathrm{mg} / \mathrm{L}$, respectively, showed a similar trend as Sample 1. The hardness was reduced by about $25 \%$ after 10 -min treatment, with an energy input of approximately $1800 \mathrm{~J} / \mathrm{L}$. In comparison to the plasma treated cases, no significant change was observed for the no-treatment cases.

Figure 6-9 presents the variations of bicarbonate ion concentration with time determined by total alkalinity test and phenolphthalein alkalinity test. Generally, the concentration of bicarbonate ion decreased during a 10-min stabilization period before reaching an asymptotic value. The concentration of both calcium ions and bicarbonate ions changed very little for energy input greater than $2 \mathrm{~kJ} / \mathrm{L}$. It was probably because the rate of precipitation reactions was limited by the diffusion rate of calcium and bicarbonate ions towards the reaction zone due to the high localization of the plasma discharge. In addition, the acidification in the vicinity of anode by plasma induced $\mathrm{H}^{+}$generation (Equations. (6-15) and (6-16)) could become detrimental to the precipitation process. 


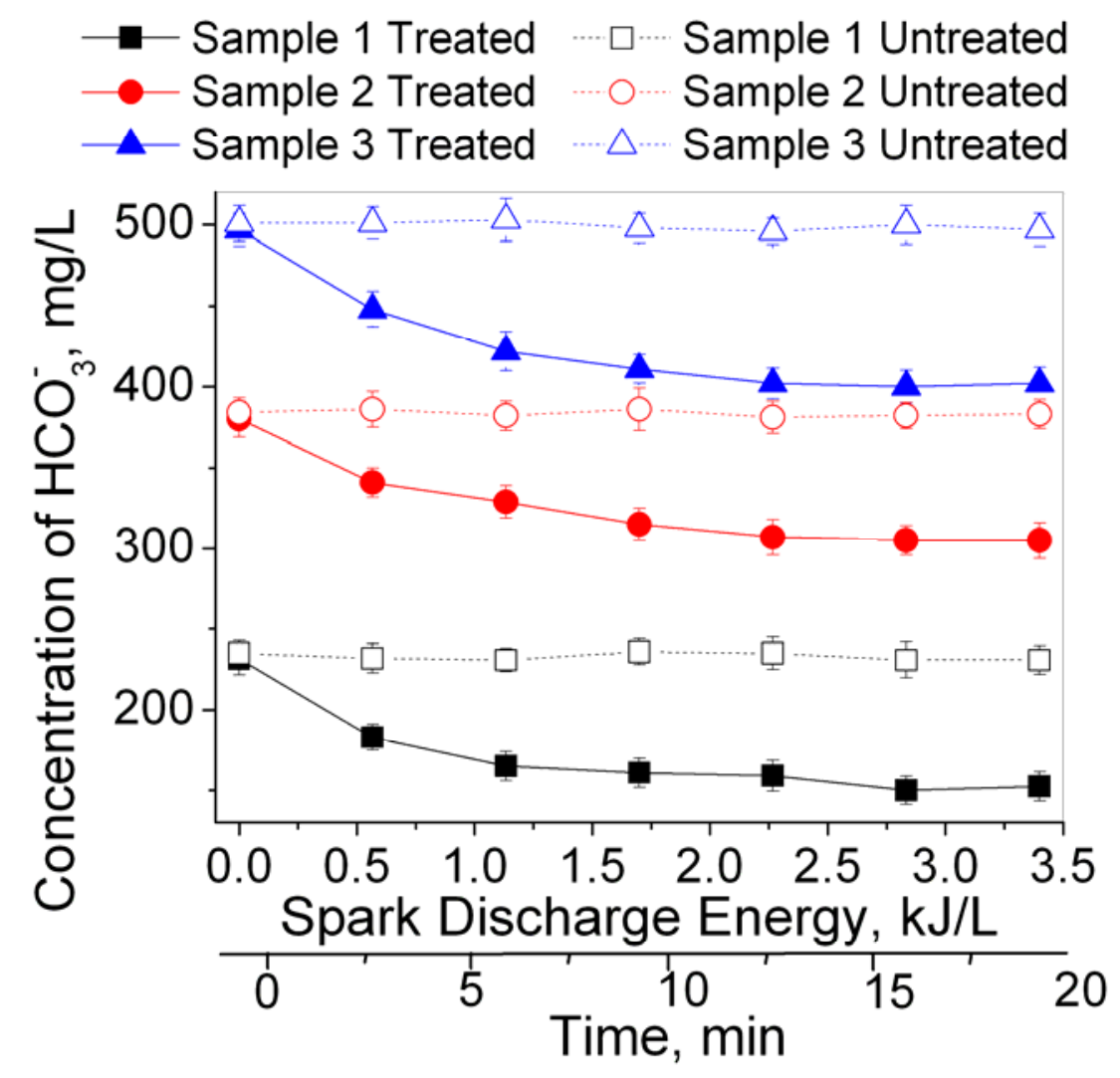

Figure 6-9. Variations of $\mathrm{HCO}_{3}^{-}$over timefor cases with and without plasma treatment (Yang et al., 2010a).

Figure 6-10 shows the number of particles per unit volume of $1 \mathrm{ml}$ as a function of particle size before and after plasma treatment, which were determined using a dynamic laser scattering system. The results depict in general that before the treatment, the number of particles less than $10 \mu \mathrm{m}$ was significantly greater than that of larger particles (greater than $10 \mu \mathrm{m}$ ). For example, for Sample 1 (Figure 6-10(a)), the number of particles with size between 1 and $2 \mu \mathrm{m}$ was 17,048 before treatment as compared to 2,445 for particles greater than $10 \mu \mathrm{m}$. After treatment, a significant increase in the number of particles was observed for all cases as compared to the notreatment cases. For Sample 1, the number of particles with size between 1 and $10 \mu \mathrm{m}$ increased from 39,904 at the initial state to 77,680 at the end of the treatment, while the number of particles with size over $10 \mu \mathrm{m}$ increased from 2445 to 3529. For Samples 2 and 3 (Figures 10(b) and $10(\mathrm{c}))$, particles smaller than $10 \mu \mathrm{m}$ also made up the majority of the suspended solids in water. The number of small particles (i.e., below $10 \mu \mathrm{m}$ ) after the treatment was significantly increased comparing with that obtained at no-treatment cases. 

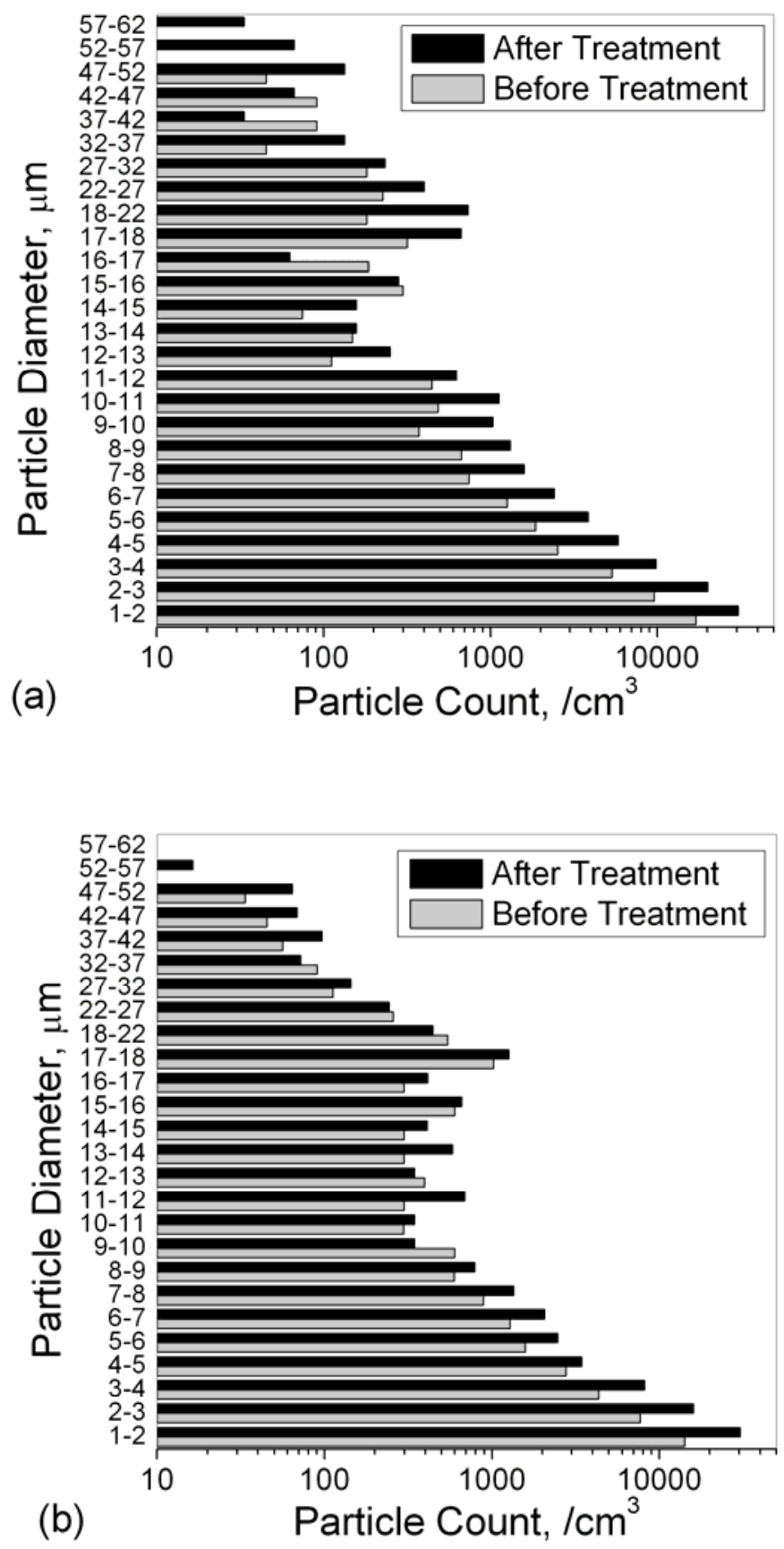


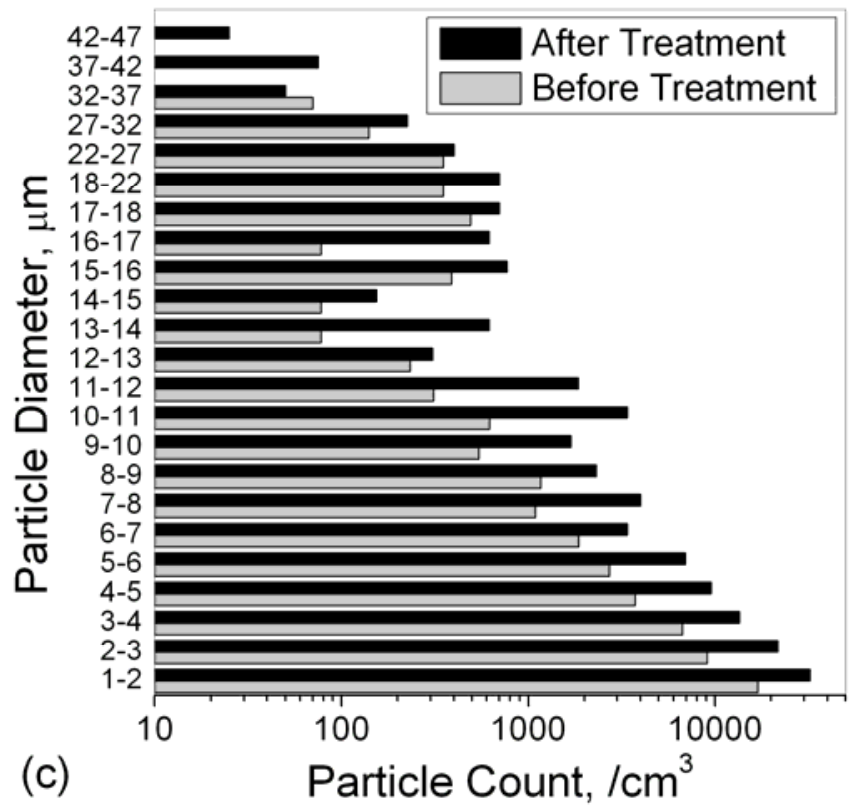

Figure 6-10. Particle size distributions before and after plasma treatment for (a) Sample 1; (b) Sample 2; (c) Sample 3 (Yang et al., 2010a).

Assuming that all the particles were in the spherical shape for the purpose of mathematical estimation, the total mass of suspended solid contents $m$ was calculated using the following equation:

$$
m=\sum_{N_{d}(d=1 \mu m)}^{N_{d}(d=62 \mu m)} \rho \cdot \frac{\pi d^{3}}{6} \cdot N_{d}
$$

where $\rho$ is the density of the $\mathrm{CaCO}_{3}$ particle, $\mathrm{d}$ is the diameter of the particle, $\mathrm{N}_{\mathrm{d}}$ is the number of particles. $\rho$ is taken as $2.7 \mathrm{~g} / \mathrm{cm}^{3}$, which is the density of calcite. For Sample 1, the total mass of solid particles before the treatment was $64 \mathrm{mg} / \mathrm{L}$, which corresponded to $64 \mathrm{mg} / \mathrm{L} \mathrm{CaCO}_{3}$ hardness. The value increased to $104 \mathrm{mg} / \mathrm{L}$ after the plasma treatment, which means that the difference of a $40 \mathrm{mg} / \mathrm{L}$ of ionic content in water was transformed from the dissolved ionic states into solid content during the process. Aforementioned titration results from Figure 6-9(a) showed that the calcium ion hardness in water was reduced by $25 \mathrm{mg} / \mathrm{L}$, equaling to $62.5 \mathrm{mg} / \mathrm{L}$ of calcium carbonate hardness. Considering that the resolution of the laser particle counter was $1 \mu \mathrm{m}$, which means that the precipitation of particles with less than 1-micron-diameter was not taken into account, the results obtained by titration and laser particle counting was in good agreement, demonstrating that the calcium ions was transformed into calcium carbonate solids during the process. For all three cases, the results are summarized in Table 6-2. 
Table 6-2. Results obtained by laser particle counting

\begin{tabular}{|l|l|l|l|}
\hline & Sample 1 & Sample 2 & Sample 3 \\
\hline Total number of particles before treat, $\mathrm{cm}^{-3}$ & 42,349 & 38,587 & 47,034 \\
\hline Mass of suspended particles before treat & 64 & 72 & 53 \\
\hline Total number of particles after treat, $\mathrm{cm}^{-3}$ & 81,710 & 71,340 & 105,846 \\
\hline Mass of suspended particles after treat & 104 & 120 & 108 \\
\hline Increase of solid mass in water & 40 & 48 & 56 \\
\hline Decrease of ionic $\mathrm{CaCO}_{3}$ hardness in water & 62 & 67 & 75 \\
\hline
\end{tabular}

All mass values are in $\mathrm{mg} / \mathrm{L}$.

Figure 6-11(a) shows the SEM photographs of particles retrieved from Sample 1 without plasma treatment. Chemical composition of the particles was analyzed by Energy Dispersion Spectrometer (EDS). Figure 6-13 shows that the particles were mainly composed of calcium carbonate, with slight amounts of sodium and magnesium, together with the platinum coated prior to the SEM observation. The crystals exhibited the morphology close to the rounded vaterite form. However, X-ray diffraction pattern, as shown in Figure 6-13(a), coincided with that of calcite. It was probably because of the thermodynamically unstable property of the vaterite crystals. The preparation of water samples usually took several days. Possibly during this period, vaterite may have recrystallized into more stable calcite crystals without changing shape.

Figure 6-11(b) shows the photographs of the particles collected from the Sample 1 after the plasma treatment. The crystals demonstrated typical rhombohedron morphology of calcite. The formation of the calcite was confirmed by XRD shown in Figure 6-13(b). The mean crystal size of precipitation test was about $5 \mu \mathrm{m}$ after $20 \mathrm{~min}$ of the plasma treatment. For the treated water with high hardness (i.e. Sample 2 and 3), calcite crystals were also observed, and their size remained almost the same as that observed in Sample 1. The fact that the total amount of precipitates was increased as indicated by the laser particle counting suggests that the number of nuclei was significantly increased. That demonstrates that the plasma had induced chemical changes in the calcareous water during the treatment, which was implied later in the nucleation process during the precipitation test. 

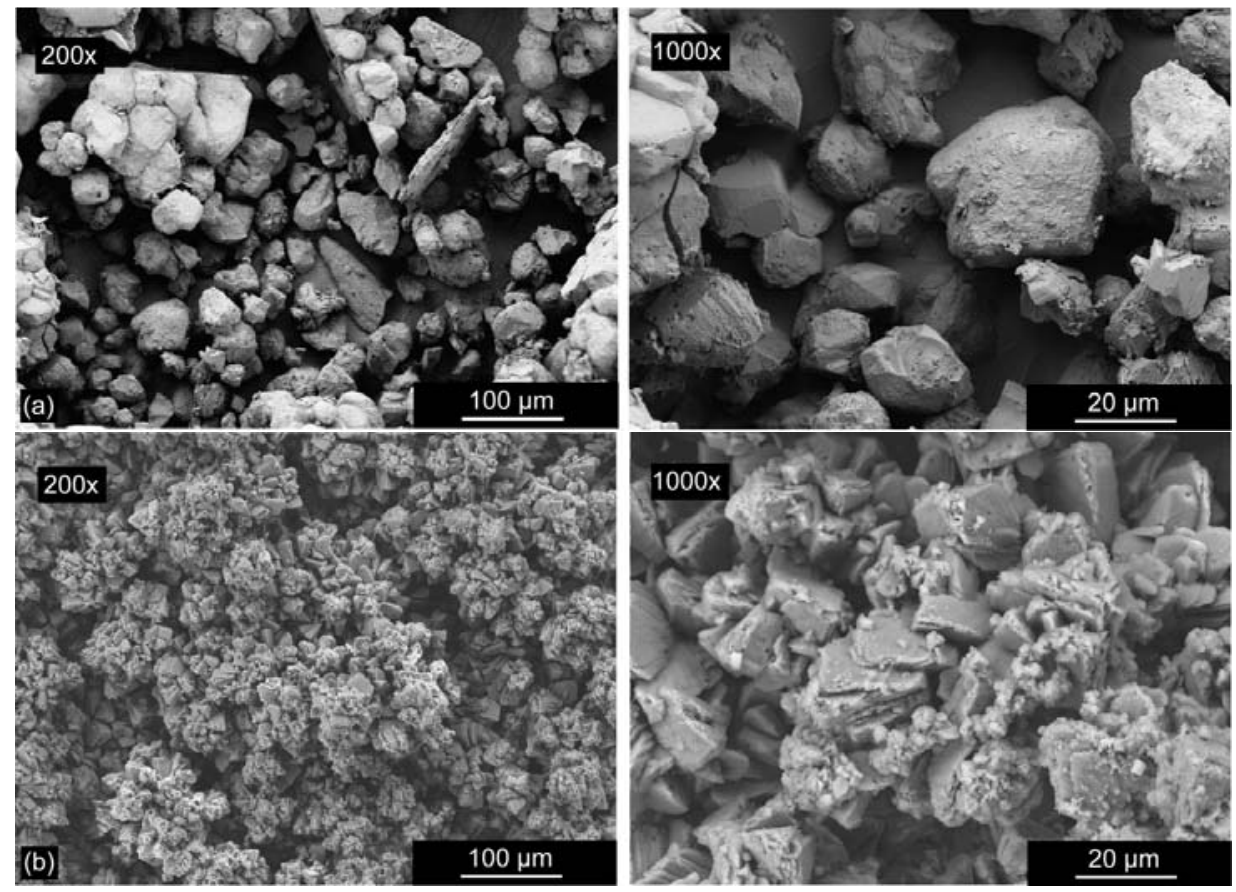

Figure 6-11. SEM images of calcium carbonate crystals obtained from: (a) untreated water: (b) plasma treated water (Yang et al., 2010a).

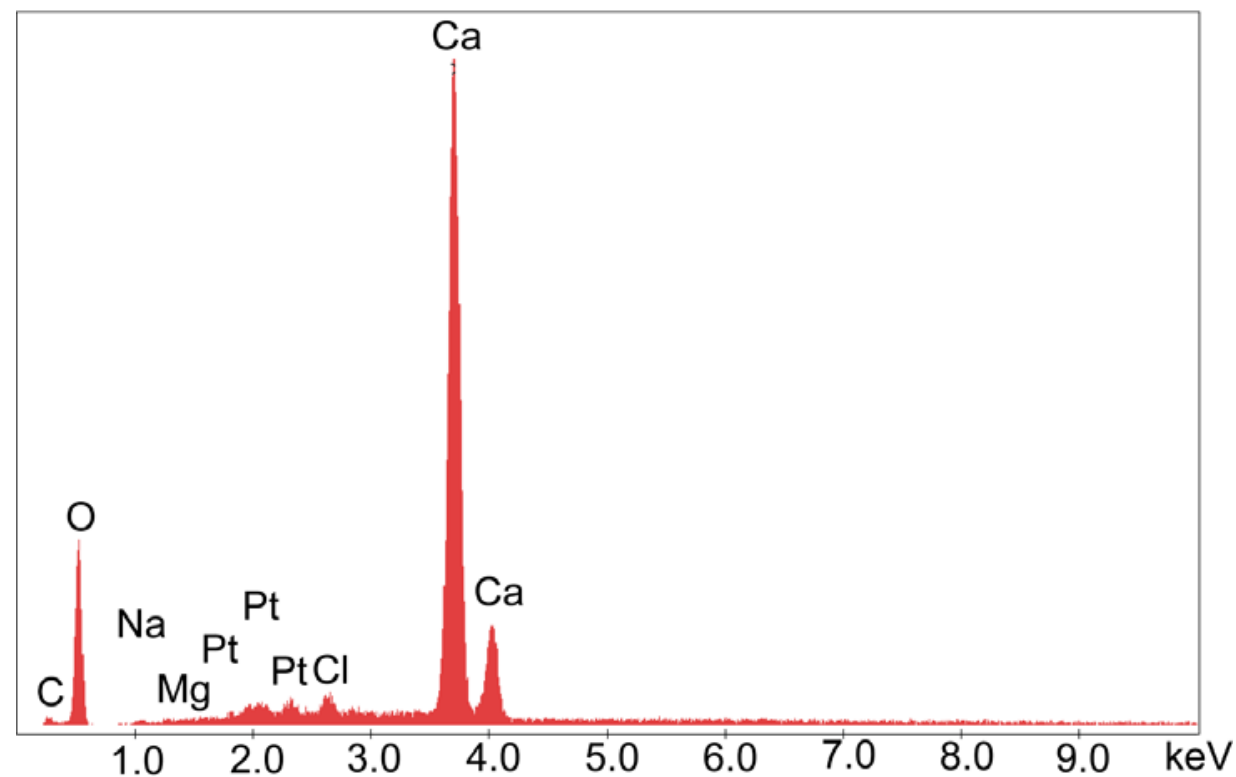

Figure 6-12. Elemental composition of the particles shown in Figure 6-11(b) obtained by Energy Dispersion Spectrometer (EDS) (Yang et al., 2010a). 


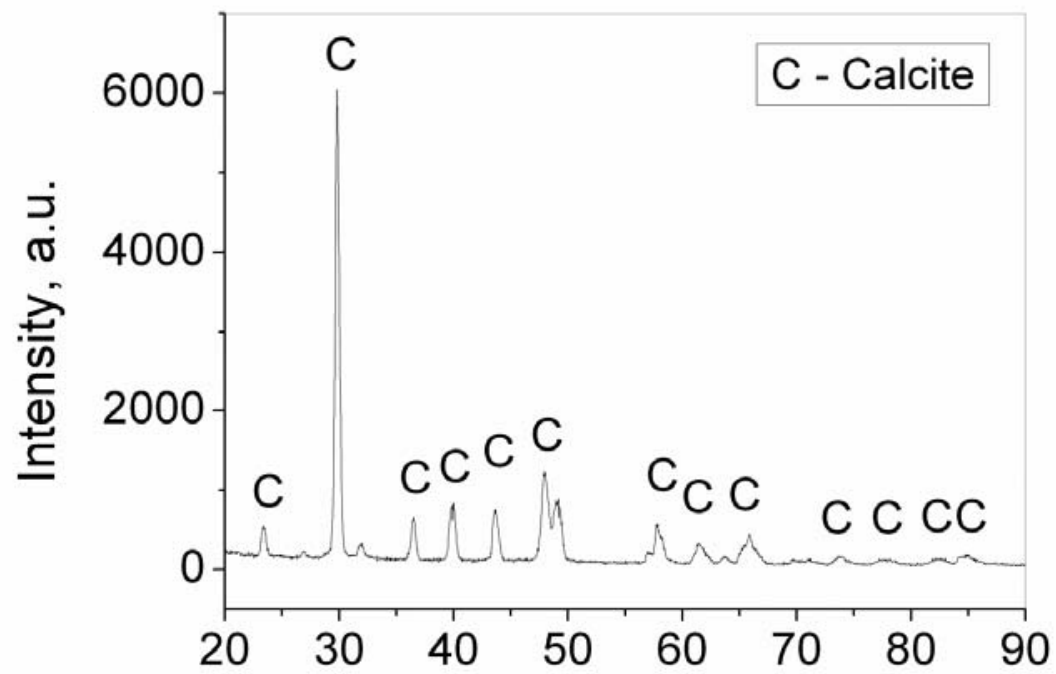

(a)

$2 \theta$

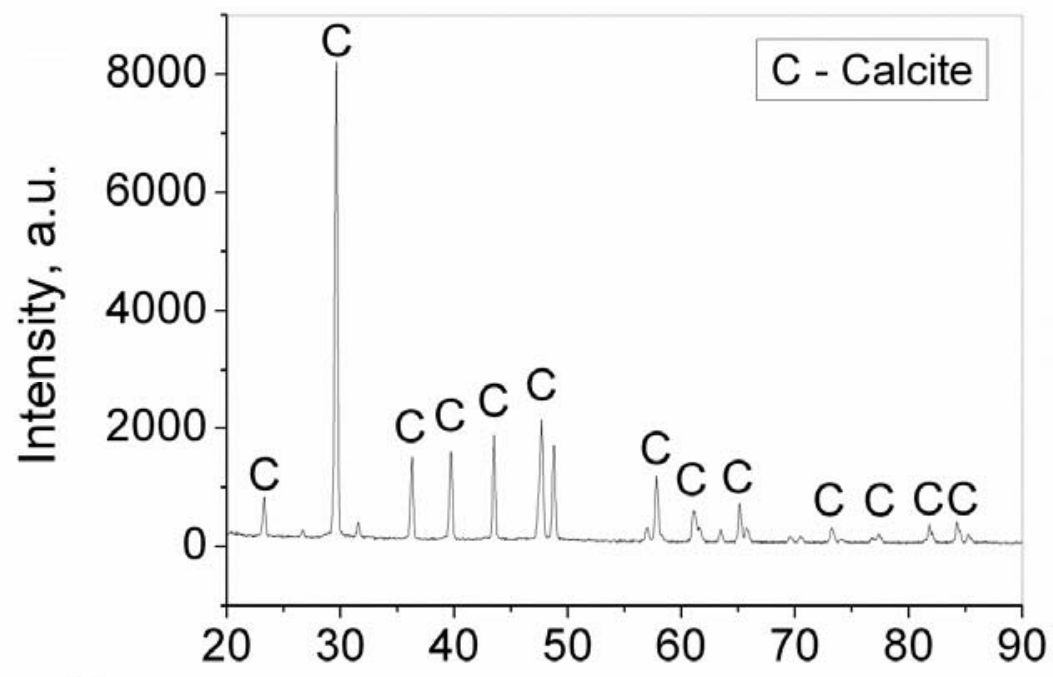

(b)

$2 \theta$

Figure 6-13. XRD pattern of the calcium carbonate crystals obtained from: (a) untreated water; (b) plasma treated water (Yang et al., 2010a). 


\subsubsection{Effect of Spray Circulation on Hardness of Cooling Water}

To disturb or eliminate the acidic condition so that a higher level of hardness reduction could be achieved than the aforementioned asymptotic hardness, the treated water sample was spray-circulated for 12 hours to degas the excessive $\mathrm{CO}_{2}$ in the plasma-treated water. For Sample 1, the $\mathrm{pH}$ value increased from 7.74 to 7.92 during this period, indicating releasing of $\mathrm{CO}_{2}$ gas from water solution. In the mean time, the hardness decreased from $190 \mathrm{mg} / \mathrm{L}$ to 160 $\mathrm{mg} / \mathrm{L}$ (see two arrows in Figure 6-14(a)). After that the water sample was treated again by the spark discharge for $20 \mathrm{~min}$, and the hardness dropped from $160 \mathrm{mg} / \mathrm{L}$ to $140 \mathrm{mg} / \mathrm{L}$ before reaching another equilibrium. At the end of a 36-hour test, the hardness reached the final asymptotic value shown in Figure 6-14(a), which indicated an approximate 45\% reduction from the initial hardness. Similar results were observed for both Samples 2 and 3 with hardness reductions of $53 \%$ and $59 \%$, respectively. For example, for Sample 3 with an initial hardness of $420 \mathrm{ppm}$, the final hardness was $170 \mathrm{ppm}$ at the end of 36-hr circulation with three brief daily plasma treatments. Note that when the plasma treatment was not used, the hardness of Sample 3 increased from 420 to $440 \mathrm{ppm}$ after 36-h circulation, 5\% increase in hardness obviously due to the evaporation of pure water as shown in Figure 6-14(a).

In summary the hardness of water could be reduced by $45-59 \%$ by the combination of plasma discharges and spray-circulation. Since the plasma treatment was applied for only 20 min in the 12-hour interval, and spray-circulation is a standard operation for cooling water in cooling tower, plasma discharge can be regarded as a "catalyst" for precipitation. 


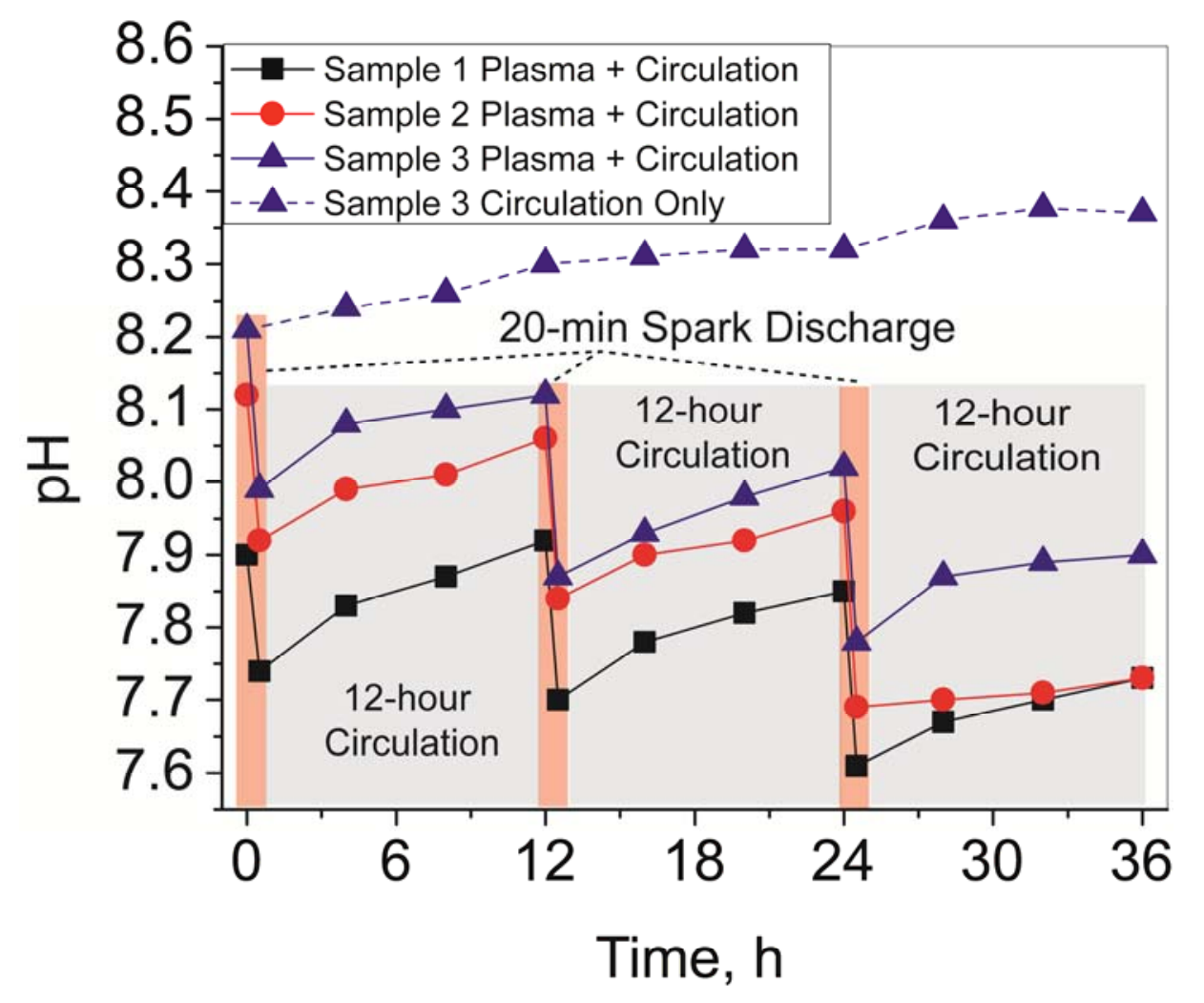

Figure 6-14. Variations of (a) $\mathrm{CaCO}_{3}$ hardness; (b) $\mathrm{pH}$ value over time with plasma treatment and spray circulation.

\subsection{Mechanism of Plasma-Induced Calcium Precipitation}

In this section, the possible mechanism for the plasma-induced calcium-carbonate precipitation is discussed.

\subsubsection{Effect of Electrolysis}

First, the effect of electrolysis, which is another hard water softening technology using electric energy, is examined. Gabrielli and his coworkers studied the principle of softening process by electrolysis and showed that the precipitation was induced by the local $\mathrm{pH}$ increase near the vicinity of the cathode (Gabrielli et al., 2006). A model electrolyser with an electrode area of $560 \mathrm{~cm}^{2}$ was tested. Under the operating current density of $20 \mathrm{~A} / \mathrm{m}^{2}$, applied voltage of $12 \mathrm{~V}$ and flow rate of $6 \mathrm{~L} / \mathrm{h}$, the $\mathrm{Ca}^{2+}$ hardness of the water sample was reduced from $96 \mathrm{mg} / \mathrm{L}$ to about $72 \mathrm{mg} / \mathrm{L}$ when a quasi-steady state operation was achieved. Hence the energy consumption was calculated as $806 \mathrm{~J} / \mathrm{L}$.

For the present spark discharge, electrolysis most likely took place during the prebreakdown stage, as the current would be transferred by the ionized gas after the formation of 
the conductive channel between the two electrodes. The same level of hardness reduction was achieved as in Gabrielli's experiments after applying spark discharge in Sample 1 for 10 min, as shown in Figure 6-9(a). The energy dissipation in the pre-breakdown stage was calculated as $984 \mathrm{~J} / \mathrm{L}$ based on the results shown in Figure 6-8. However, it was difficult to estimate the exact amount of energy consumed by electrolysis, as multiple physical and chemical processes, including ionization, light emission, cavitation, shockwave and reactive species formation, were initiated during the same stage. But certainly, only a fraction of the $984 \mathrm{~J} / \mathrm{L}$ energy was dissipated in electrolysis.

Another factor that may subdue the effect of electrolysis is the mixing of the liquid caused by the formation of shockwaves. It may prohibit the formation of local high $\mathrm{pH}$ area near the vicinity of the cathode. Therefore, the electrolysis-induced-precipitation might be suppressed in the present experiment. Hence it could be concluded that the reduction of water hardness may not be the sole effect of the electrolysis, though it may contribute partially to the precipitation process.

\subsubsection{Effect of UV Radiation}

First, the effect of UV radiation on the precipitation process of calcium ions was studied. For spark discharges, the high temperature plasma channel can function as a blackbody radiation source. The maximum emittance is in the UVA to UVC range of the spectrum produced by the spark discharge $(200 \mathrm{~nm}-400 \mathrm{~nm})$, as determined by the Stephen-Boltzmann law. Water is relatively transparent to UV radiation in this wavelength range. The energy per photon ranges from $3.1 \mathrm{eV}$ to $6.2 \mathrm{eV}$, indicating the possibility of $\mathrm{HCO}_{3}{ }^{-}$dissociation through UV absorption. Severe mineral fouling was usually observed at the quartz sleeve of commercial UV lamps, where the build-up of the fouling material could be associated with the reactions between dissolved ions and UV radiation, although the mechanism was not fully understood (Lin et al., 1999; Wait, 2005).

To investigate this mechanism of the reaction between dissolved mineral ions and UV radiation, the present study constructed a special discharge chamber where a point-to-plane electrode system was placed in a quartz sleeve filled with distilled water, as shown in Figure 615. The diameter of the quartz sleeve was $25.4 \mathrm{~mm}$ to avoid possible damages by shockwaves produced by the spark discharge. The quartz sleeve was found to provide a good UV window for the water samples while effectively trapping various chemical reactive species, heat and other effects. Before each test, the quartz sleeve was cleaned using $0.1 \mathrm{~N} \mathrm{HCl}$ solution to remove any possible $\mathrm{CaCO}_{3}$ deposit from previous experiments. Test water samples were circulated outside the quartz sleeve and treated by the spark discharge produced inside the quartz sleeve for the same time periods as in the previous experiments. The results were compared to those obtained for the cases without the quartz sleeve, and no hardness reduction was observed for the samples treated by plasma separated by the quartz sleeve. This indicates that the process of calcium-ion precipitation was not triggered by the transfer of energy to water sample through UV radiation. 


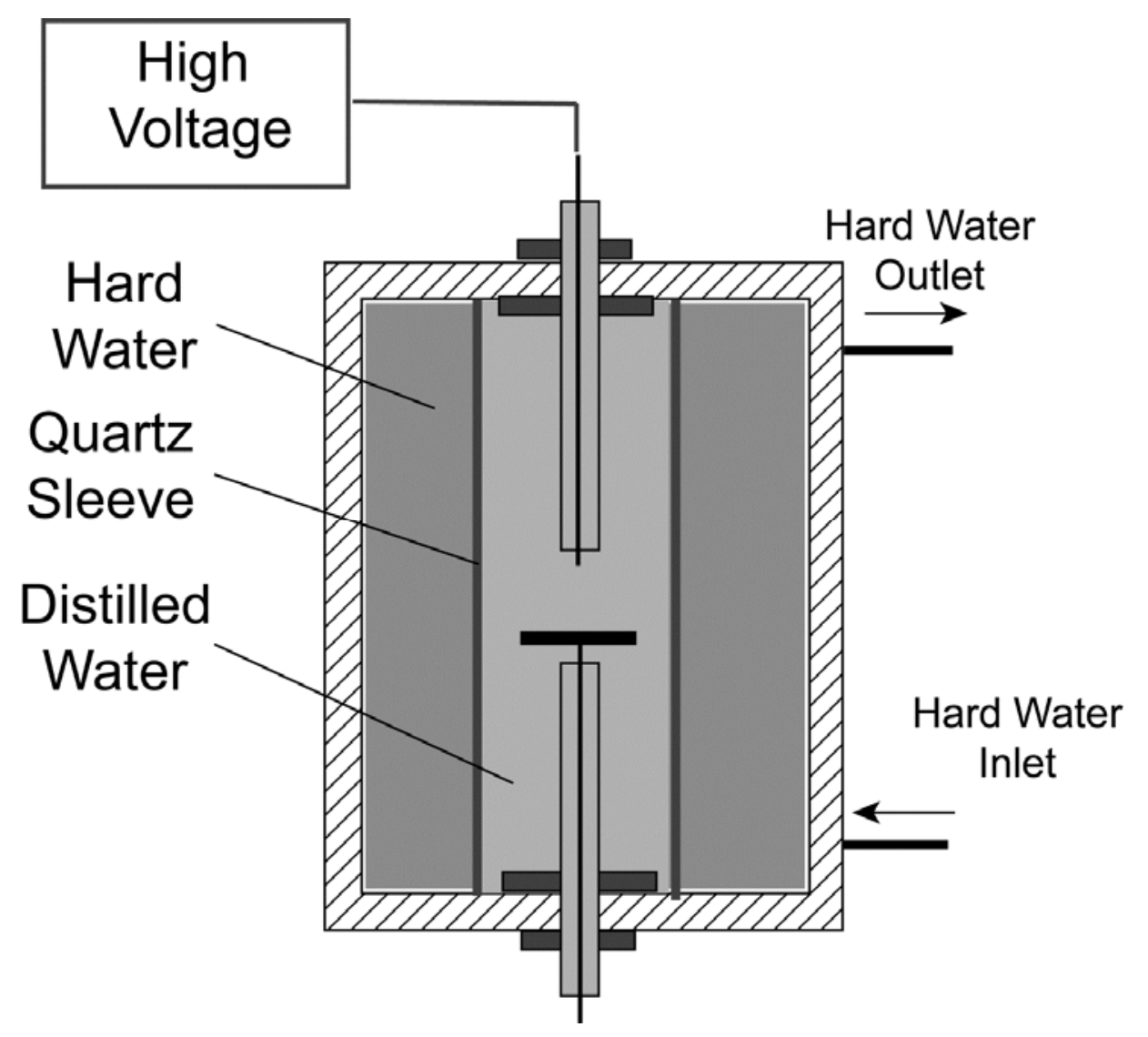

Figure 6-15. Modified discharge chamber to test the effect of $\mathrm{UV}$ on the precipitation of $\mathrm{CaCO}_{3}$.

\subsubsection{Effect of Reactive Species}

From the emission spectrum of the pulsed discharge in distilled water, the formation of hydroxyl radicals and other reactive species by underwater plasma process was reported (Sunka et al., 1999; Joshi et al., 1995; Sun et al., 1998). In case of hard water, OH radicals would react with bicarbonate ions to produce carbonate radicals $\mathrm{CO}_{3}^{*-}$, through the following reaction (Buxton and Elliot, 1986; Crittenden et al., 1999):

$$
\mathrm{HCO}_{3(\mathrm{aq})}^{-}+\mathrm{OH}_{(\mathrm{aq})}^{*} \Leftrightarrow \mathrm{H}_{2} \mathrm{O}+\mathrm{CO}_{3(\mathrm{aq})}^{*-}\left(\mathrm{k}=8.5 \times 10^{6} \mathrm{M}^{-1} \mathrm{~s}^{-1}\right)
$$

Being a highly oxidizing species, $\mathrm{CO}_{3}^{*-}$ is known to be active in the oxidation of some organic compounds by direct electron transfer to produce the corresponding cation radical and carbonate anion (Crittenden et al., 1999) as shown by the following equation:

$$
\mathrm{CO}_{3(\mathrm{aq})}^{*-}+\mathrm{R} \Leftrightarrow \mathrm{R}^{*+}+\mathrm{CO}_{3(\mathrm{aq})}^{2-}
$$


Hence, it is hypothesized that the reactive species produced by the spark discharge in hard water may transform the bicarbonate ions into carbonate ions without going through Equations (6-7) and (6-8). To test this hypothesis, multiple layers of polyethylene meshes were placed close to the electrodes. Polyethylene is one of the most commonly used materials for activespecies scavenger in water. Typical rates of the reaction between the $\mathrm{OH}$ radical and organic materials are in the order of $10^{9}-10^{10} \mathrm{M}^{-1} \mathrm{~s}^{-1}$, about 2 orders of magnitude higher than that of the reaction between $\mathrm{OH}$ radicals and bicarbonate ions. Thus, the polyethylene mesh could serve as an effective scavenger screen to the radicals. The water samples were circulated and treated for the same time period with all the other experimental parameters remained the same as in the previous experiments. No significant difference was observed in the calcium-ion concentration as compared to the results shown in Figure 6-9. Therefore, it was also concluded that the reactive species were not responsible for the plasma-induced calcium-carbonate precipitation.

\subsubsection{Effect of Micro-Heating}

To gain further insight into the structure of the precipitated calcium-carbonate particles, morphological and crystallization examinations were performed by SEM and XRD, respectively. Figure 6-12 shows the particles retrieved from the untreated water sample. The crystals exhibited the morphology similar to a round-shaped vaterite, although XRD data showed that the particles were in a calcite form (not shown). Figure 6-5(b) shows the particles retrieved from the plasma-treated water sample. The crystals demonstrated a typical rhombohedron morphology of calcite, suggesting different mechanisms of precipitation after plasma treatment. Natural calcium-carbonate precipitation is a complex process that has been studied extensively. It is known that calcium carbonate exists in three crystalline polymorphs with different structures: calcite, aragonite and vaterite. Among them, calcite is the most thermally stable form, and is the dominant polymorph of $\mathrm{CaCO}_{3}$ formed by the loss of carbon dioxide or evaporation of natural calcium bicarbonate solutions if temperature is the controlling factor (Siegal and Reams, 1966). The other two crystalline forms are metastable phases of calcium carbonate, which would transform to calcite spontaneously under normal conditions. The transformation process would be expedited on heating. Therefore, from the fact that calcite with a rhombohedron morphology was formed during the plasma-assisted precipitation process, one may hypothesize that the precipitation may be temperature related.

Direct proof of the validity of this hypothesis is difficult, as it is not easy to produce a local heating zone with temperature up to 5,000-10,000 $\mathrm{K}$ in water without inducing other effects. In order to test this hypothesis, the present study utilized a transient hot-wire method. When a thin wire immersed in a sample liquid is heated by electrical current (i.e., joule heating), the wire can play as an electrical heating element and produce an elevated temperature in the surrounding water with. The transient hot-wire technique is widely employed today for the measurement of the thermal conductivity of fluids over a wide range of temperatures (Nagasaka 
and Nagashima, 1981; Xie et al., 2006). Although the temperature rise in the conductivity measurement is usually much lower than that in the spark discharge channel, the hot-wire method is still a good approximation as the duration of the high temperature produced by the spark discharge is relative short (usually in $\mu$ s) and the intense local heating would quickly be dissipated through the conductive and convective heat transfer in water.

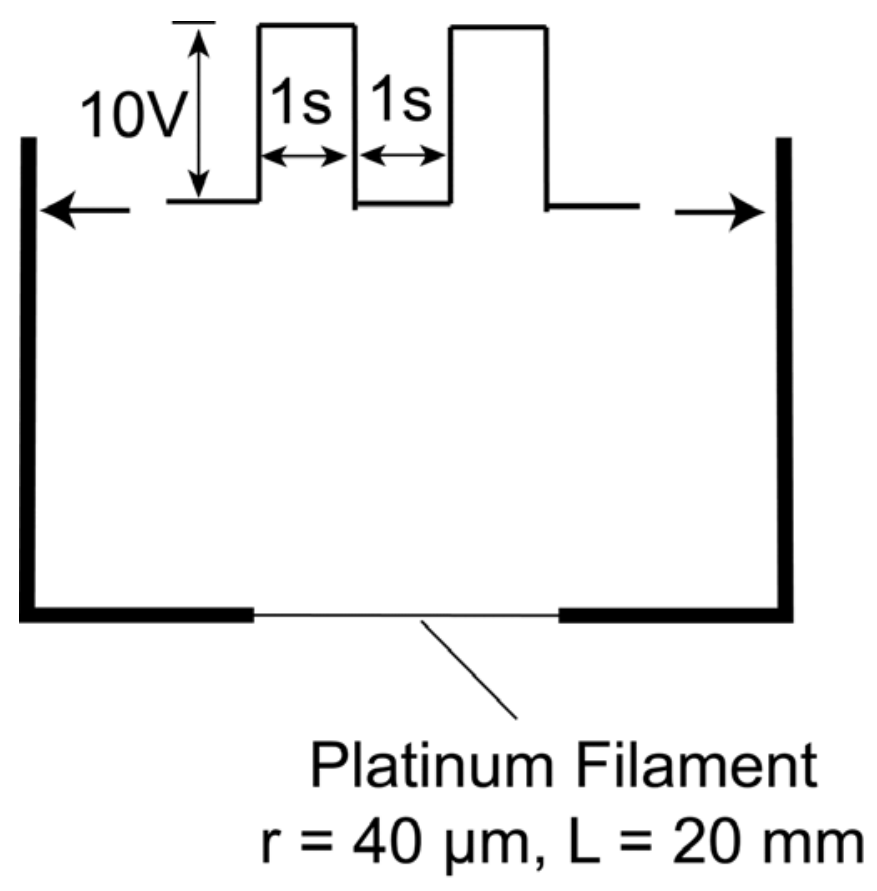

Figure 6-16. Schematic diagram and voltage waveform used in the present transient hot-wire method. (Yang et al., 2011b).

A platinum wire of $80 \mu \mathrm{m}$ in diameter, $20 \mathrm{~mm}$ in length was used in the present study. The resistance of the effective length of the wire was about $10 \Omega$ at $25^{\circ} \mathrm{C}$. The power for the circuit for heating the wire was provided by a square-wave alternating voltage unit, with the voltage waveform shown in Figure 6-16. The wire was heated during a duty cycle and cooled during an off-duty cycle when $\mathrm{V}=0$. The temperature rise of the wire $\Delta \mathrm{T}$ can be given by the following equation (Assael et al., 1998):

$$
\Delta \mathrm{T}=(\mathrm{q} / 4 \pi \lambda) \ln \left(4 \kappa \mathrm{t} / \mathrm{a}^{2} \mathrm{C}\right)
$$

where $\lambda$ and $\kappa$ are the thermal conductivity and diffusivity of the liquid, respectively, $\mathrm{q}$ is the heat generation per unit length of the wire, $\mathrm{a}$ is the radius of the wire, $\mathrm{C}=\mathrm{e}^{\mathrm{y}} \approx 1.781$, $\mathrm{y}$ is Euler's constant, and $\mathrm{t}$ is the time of heating. With $\mathrm{R}=10 \Omega$ and $1=20 \mathrm{~mm}$, q can be calculated as 


$$
\mathrm{q}=\mathrm{V}^{2} / \mathrm{R} / \mathrm{l}=2.5 \times 10^{4} \mathrm{~W} / \mathrm{m}
$$

Hence, the temperature rise at the end of heating period was about $825 \mathrm{~K}$. The actual temperature rise would be much lower than the theoretical value due to the boiling of water. During the cooling period, the time constant for cooling can be given as (Incropera et al., 2007):

$$
\tau=\frac{\rho V c_{p}}{h A}
$$

where $\rho, V, c_{p}$ and $A$ are the density, volume, specific heat and surface area of the platinum wire, $h$ is the convective heat transfer coefficient of water. Substituting the values for $\rho, V, c_{p}$ and $A$ into Equation (6-22), one can get

$$
\tau \approx \frac{100}{\mathrm{~h}} \mathrm{~s}
$$

For water, the typical convective heat transfer coefficient $\mathrm{h}$ is $300-10,000 \mathrm{~W} /\left(\mathrm{m}^{2} \mathrm{~K}\right)$ (Incropera et al., 2007), which means under normal conditions, the time constant for cooling $\tau$ is significantly less than $1 \mathrm{~s}$, and the wire would be cooled to room temperature during the cooling period.

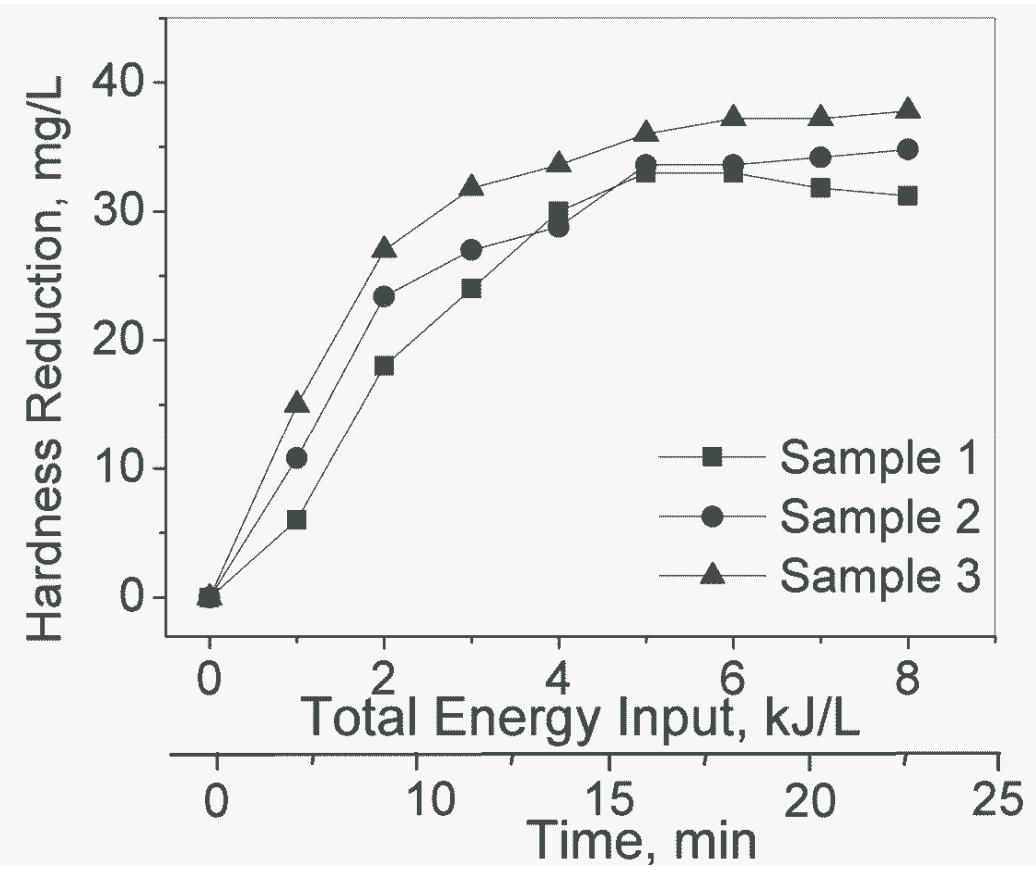

Figure 6-17. Calcium carbonate hardness reduction versus input energy by the transient hot-wire method. (Yang et al., 2011b). 
Figure 6-17 shows the effect of transient hot wire on the changes in calcium-ion concentration for three different water samples. For all three cases, about $15 \%$ hardness decrease was observed for the similar level of energy input despite the much lower temperature gradient compared to that in the plasma treatment cases. Considering the maximum $25 \%$ hardness drop in the case of the pulse spark discharge, one can conclude that the local microheating can be one of the major pathways to the precipitation of calcium ions in hard water. White deposits were observed on the filament immediately after the application of the pulsed voltage. At $\mathrm{t}=15 \mathrm{~min}$, the filament was fully covered by calcium carbonate scale, when hardness reduction reached an asymptotic value. It could be attributed to the fact that the wire could not function as a hot surface in water anymore because of the accumulation of the thermal-insulating layer of $\mathrm{CaCO}_{3}$ scale. The scale on the filament was examined using SEM and XRD. Rhombohedral shape calcite was observed, which was similar to the shape of calcium carbonate particles collected in the plasma treatment cases. Figure 6-19 shows the number of particles per $1 \mathrm{ml}$ water sample as a function of particle size before and after the hot-wire treatment for Sample 1. No significant increase in the number of particles was observed as compared to the no-treatment cases, possibly due to the fact that most of the calcium carbonate precipitated on the hot-wire surface instead of in bulk water.

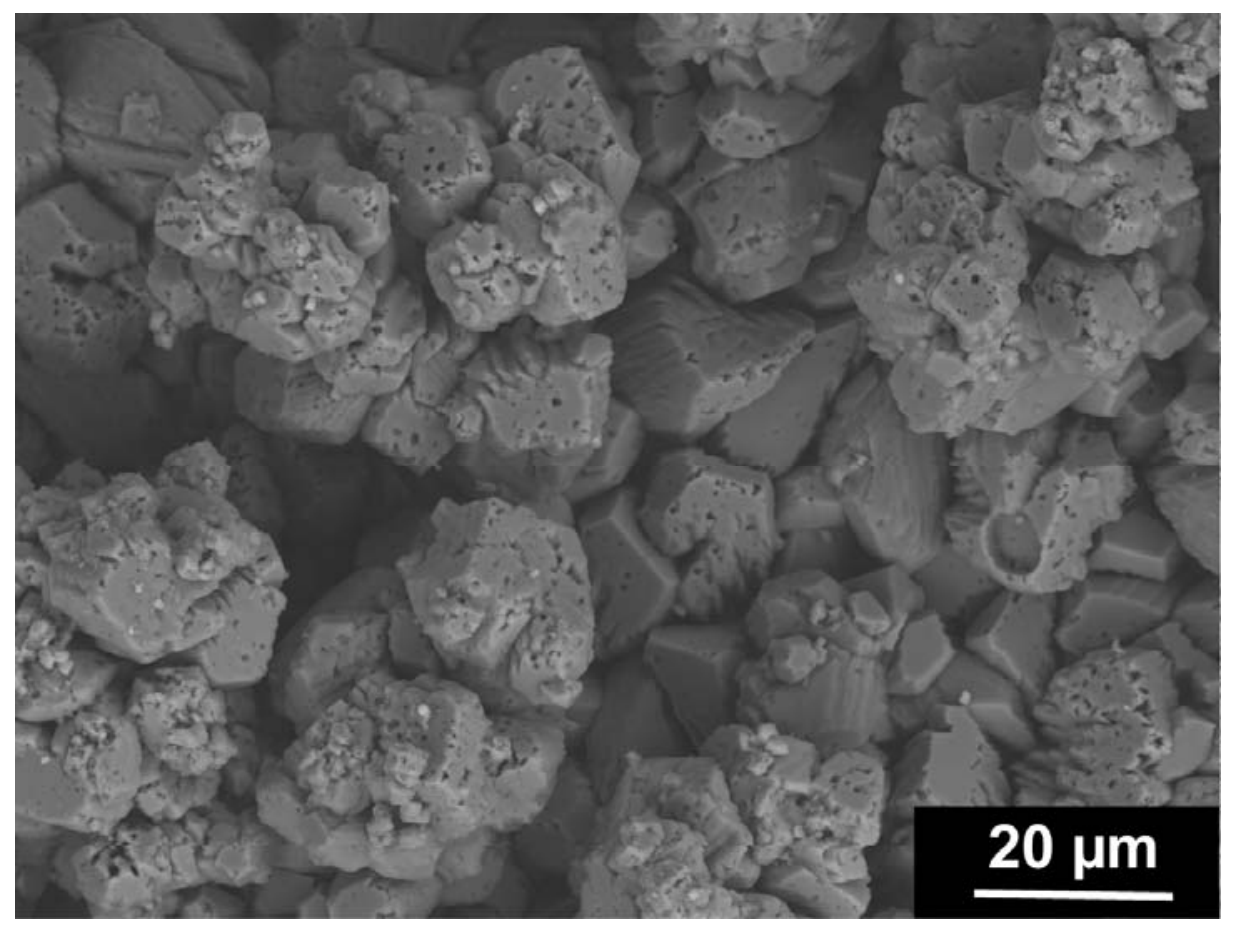

Figure 6-18. SEM images of calcium carbonate scale obtained from hot wire surface (Yang et al., 2011b) 


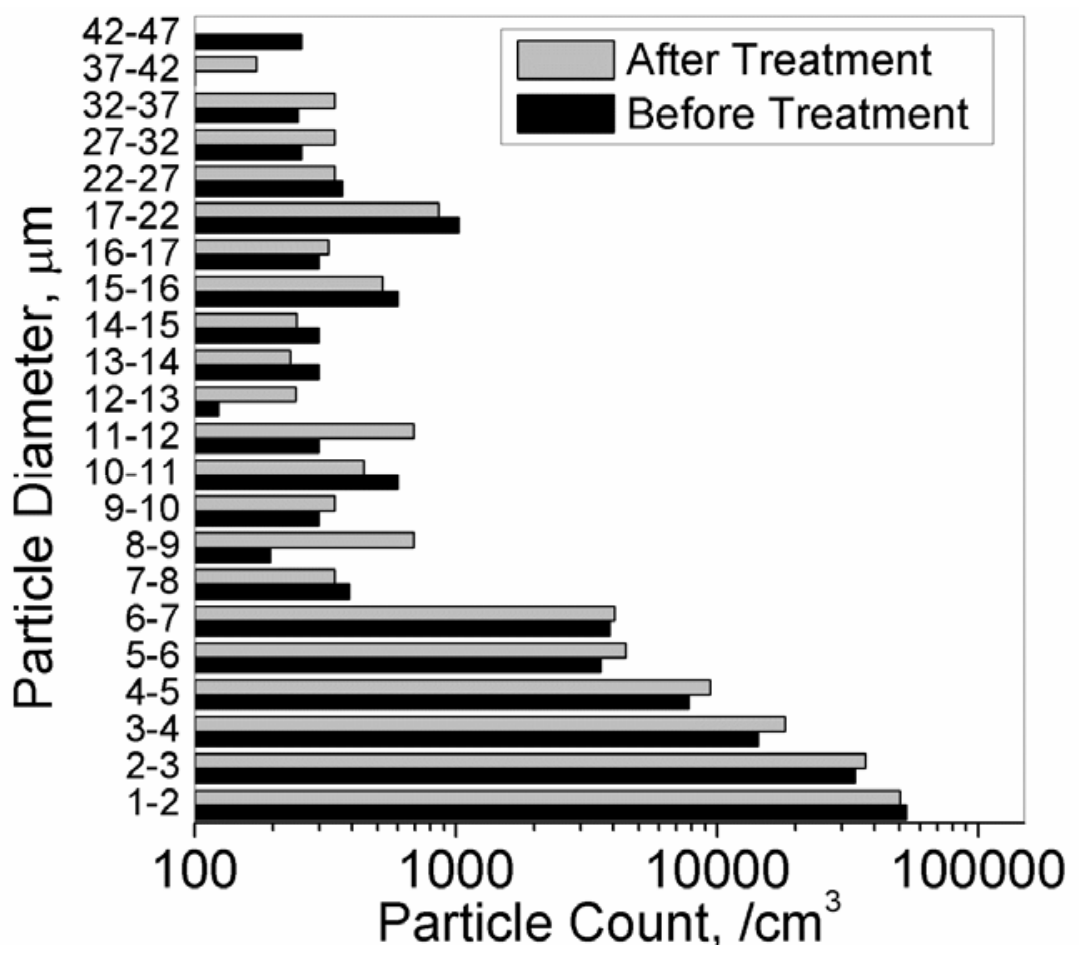

Figure 6-19. Particle size distributions before and after transient hot wire treatment (Yang et al., 2011b).

\subsubsection{Non-thermal Effect of Plasma}

To study the influence of other products produced by plasma discharge and avoid the introduction of any thermal effects, a nanosecond pulsed power supply was constructed by adding the second spark gap in the circuit as shown in Figure 6-20. The second spark gap was built with an adjustable inter-electrode distance to adjust the duration of the pulse so that one could remove the voltage from the load. The typical voltage and current waveforms are shown in Figure 6-21. The pulsed source provided $20 \mathrm{kV}$ pulses with about $10 \mathrm{~ns}$ in duration at a repetition rate of approximately $38 \mathrm{~Hz}$. Details of the corona discharge produced were reported elsewhere (Staack et al., 2008). Spectroscopy measurement through the expansion of $\mathrm{H} \alpha$ emission line demonstrated an almost non-thermal condition inside the plasma channel, confirming that the effect of micro-heating on the precipitation of calcium ions was eliminated. 


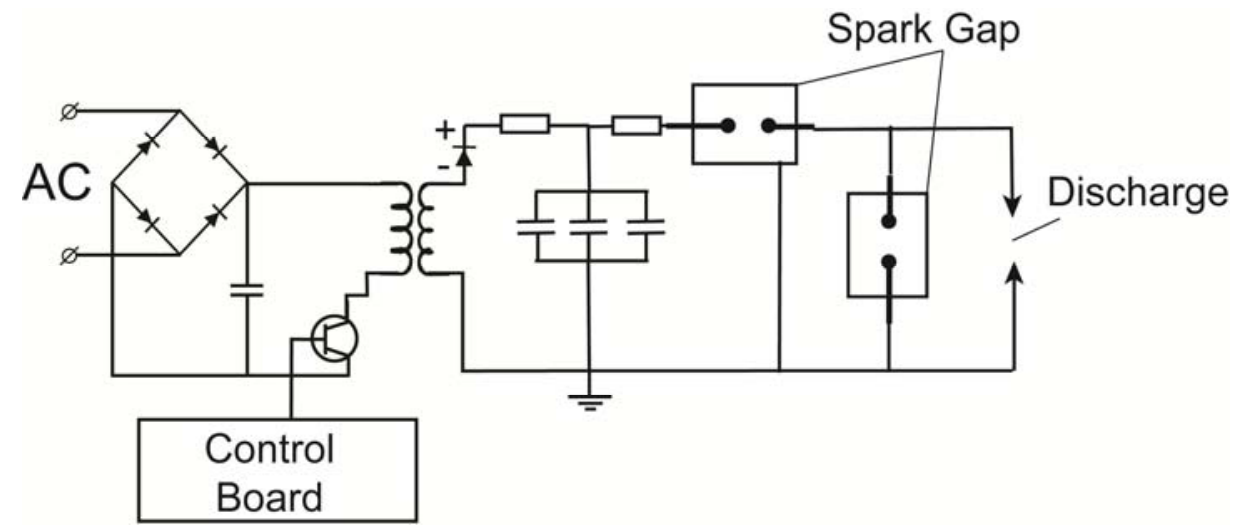

Figure 6-20. Schematic diagram of the double spark gaps configuration in the present study.

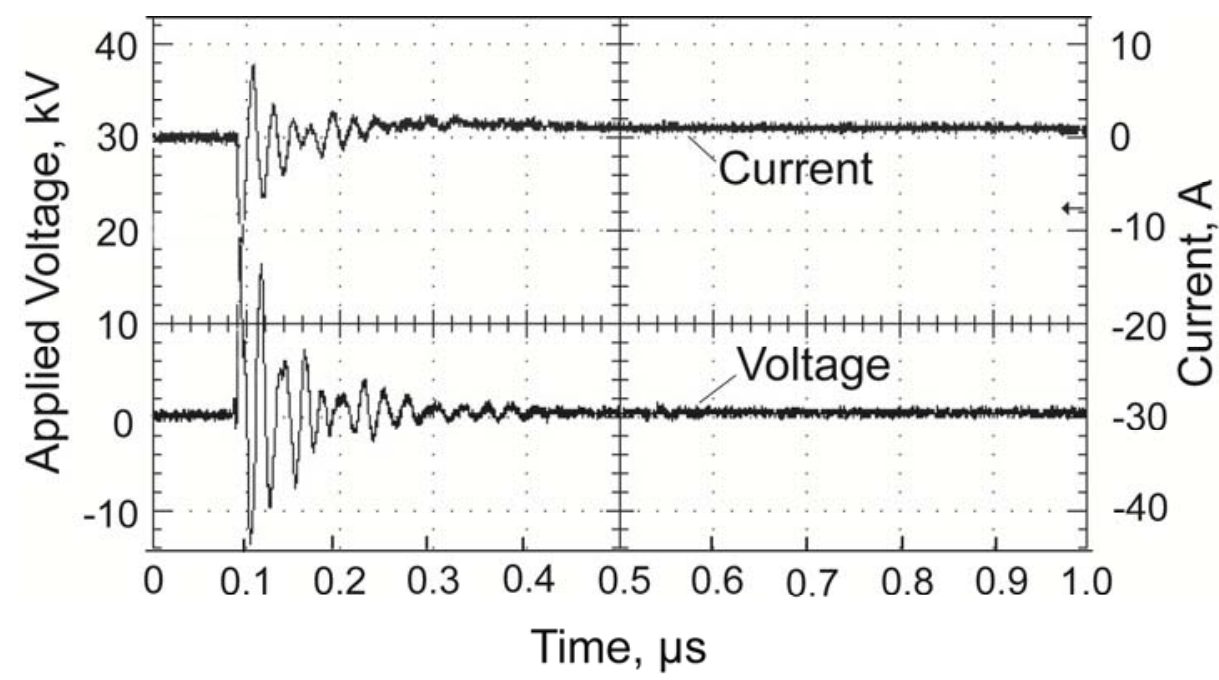

Figure 6-21. Typical voltage and current waveform produced by the circuit in the double spark gap configuration in the present study.

Figure 6-22 shows the changes in calcium-ion concentration with the application of the nanosecond corona discharge. Maximum 7\% drop in the hardness was observed, demonstrating the possibility to trigger the precipitation process through non-thermal discharges. The precipitation rate decreased with time, reaching zero at $\mathrm{t}=20 \mathrm{~min}$. After that, the hardness value began to increase, possibly because precipitated calcium carbonate particles began to redissolve due to the ionization of water molecules and subsequent acidification of the solution, as illustrated by Equations. (6-15) and (6-16). Drop in $\mathrm{pH}$ was observed for all three water samples as shown in Figure 6-22.

Figure 6-23 shows the number of particles per $1 \mathrm{ml}$ water sample as a function of particle size before and after treatment. A significant increase in the number of particles, especially for particles with diameter below $10 \mu \mathrm{m}$ size, was observed, indicating the occurrence of precipitation in bulk volume. The particles suspended in the bulk volume were collected and 
examined using both SEM and XRD. Figure 6-24 shows a mixture of irregular and rhombohedral shaped calcite. The former was probably pre-existing in the water sample before the plasma treatment. The rhombohedral shaped calcite was probably formed during the plasma treatment, which was in similar polymorph with calcium carbonate particles collected in the regular spark discharge treatment.

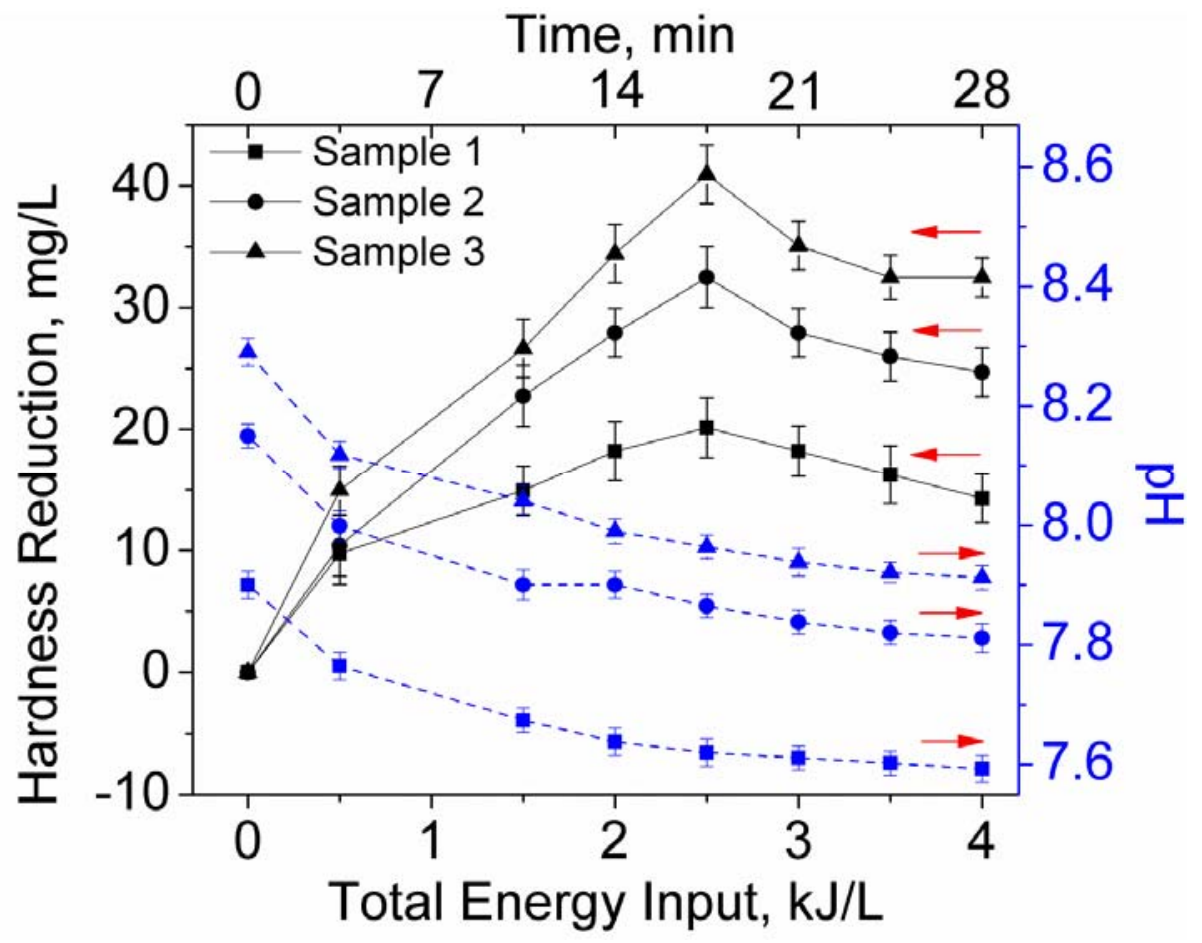

Figure 6-22. Variations of calcium carbonate hardness and $\mathrm{pH}$ over time for different energy inputs by the transient hot wire method (Yang et al., 2011b). 


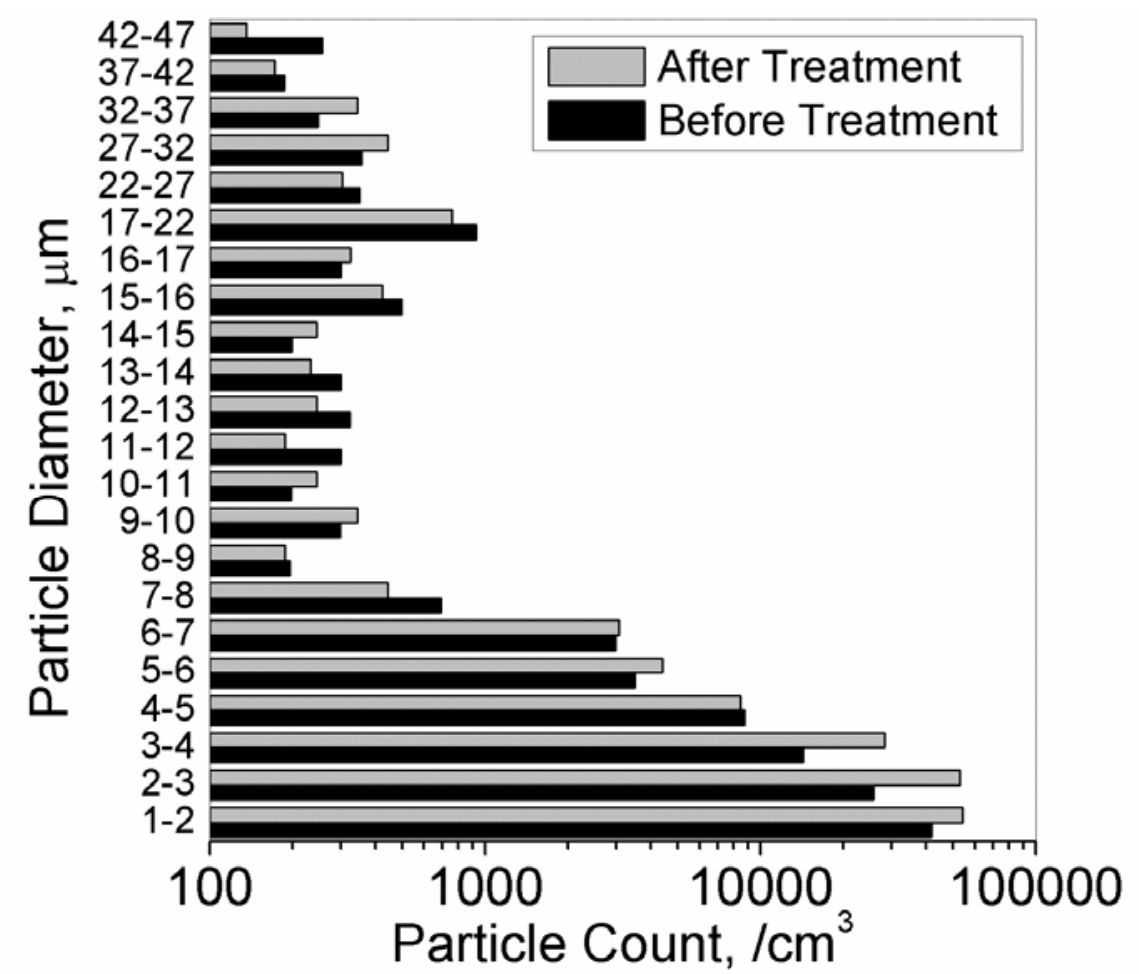

Figure 6-23. Particle size distributions before and after pulsed nanosecond discharge treatment (Yang et al., 2011b).

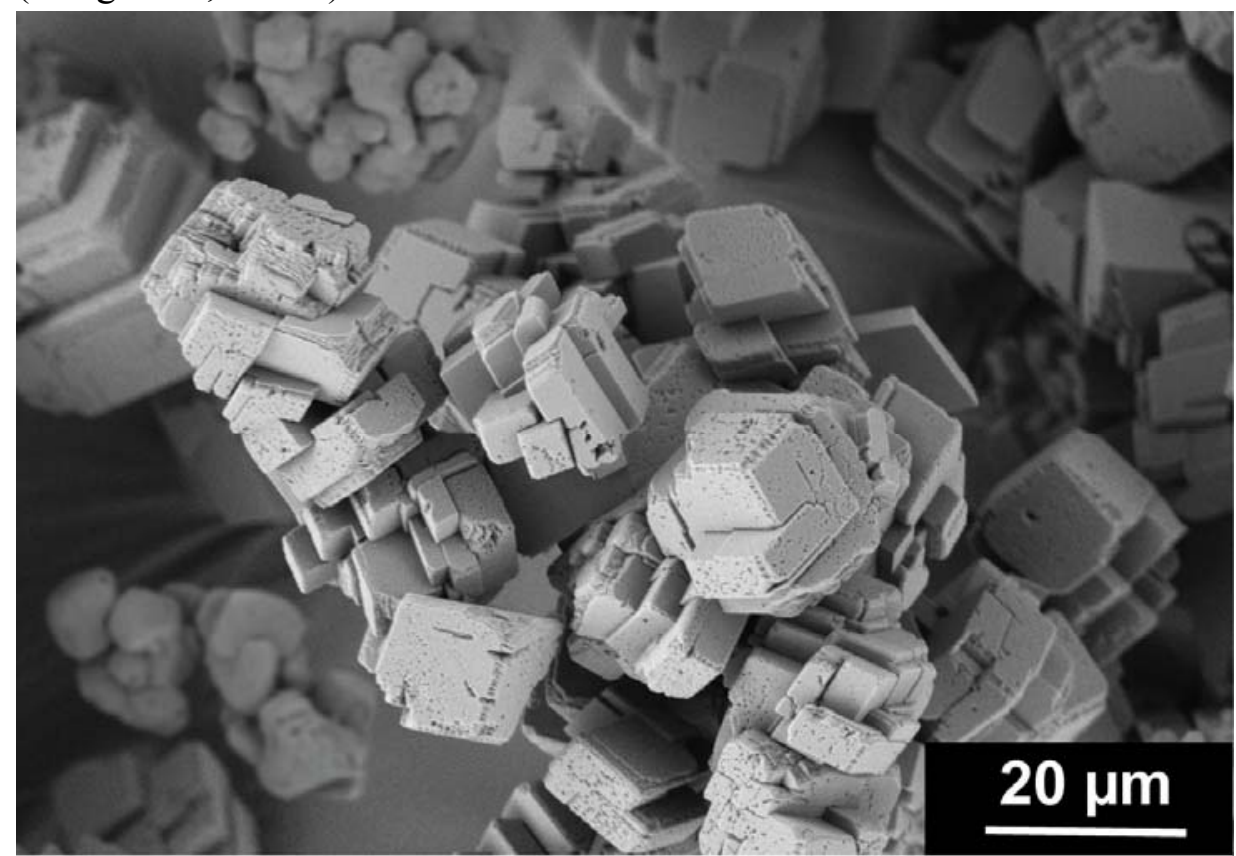

Figure 6-24. SEM image of calcium carbonate particles obtained from water sample treated by pulsed nanosecond discharge (Yang et al., 2011b). 


\subsubsection{Discussions on Calcium Precipitation with Plasma}

The present study proposes that the local micro-heating is the primary mechanism in the calcium hardness reduction by spark discharges. One can easily precipitate supersaturated calcium ions in hard water by simply increasing water temperature (i.e., volume heating). Technically sound and simple, but it is not a real solution due to the high cost associated with the volume heating. Hence, instead of the volume heating, the present study attempted to dissociate the bicarbonate ions using spark discharges in water, which is referred as a local micro-heating.

For comparison, one can calculate the numbers of $\mathrm{OH}^{-}$from both the volume heating and local heating in order to see the benefit of the local heating in the precipitation of calcium ions. The amount of $\mathrm{OH}^{-}$per unit time which one can produce from the dissociation reaction of bicarbonate ion, Equation (6-9), is calculated as (Fridman and Kennedy, 2006):

$$
n_{\mathrm{OH}^{-}}=n_{\mathrm{HCO}_{3}^{-}} \times k
$$

where $n_{\mathrm{HCO}_{3}^{-}}$is the number of $\mathrm{HCO}_{3}{ }^{-}$participating in the reaction, and $\mathrm{k}$ is the reaction rate coefficient. According to the Arrhenius equation, the reaction rate coefficient $\mathrm{k}$ becomes:

$$
k=A e^{-E_{a} / T}
$$

where $\mathrm{E}_{\mathrm{a}}$ is activation energy, $\mathrm{T}$ is the system temperature (in the unit of $\mathrm{eV}$ ). Due to the exponential curve of the equation, the Arrhenius equation indicates that the higher the water temperature is, the faster the reaction will be. The present study utilized spark discharges in water. Hence, one can expect a very intense local heating of a small volume of water around the tip of the electrode, significantly raising the temperature of the small volume of water near the tip.

The important scientific issue is whether the spark discharge used in the present study can dissociate $\mathrm{HCO}_{3}{ }^{-}$without spending a large amount of electrical energy. One can examine two cases (i.e., volume heating and local heating) to find out which case produces more $\mathrm{OH}^{-}$for the exactly same amount of energy spent.

For the volume heating, one can assume to heat the entire volume of water by one degree (e.g. from 300 to $301 \mathrm{~K}$ ). Then, the number of $\mathrm{OH}^{-}$one can produce for $\mathrm{E}_{\mathrm{a}} \approx 1 \mathrm{eV}$ becomes:

$$
n_{\mathrm{OH}^{-}}=n_{\mathrm{HCO}_{3}{ }^{-}} \times k=n_{\mathrm{HCO}_{3}^{-}} \times A e^{-E_{a} / T}=\mathrm{An}_{\mathrm{HCO}_{3}{ }^{-}} e^{-11000 / 301}=e^{-36.5} \mathrm{An}_{\mathrm{HCO}_{3}{ }^{-}}
$$

For the local heating using spark discharge, one can assume to heat $1 \%$ of the entire water volume by 100 degrees (e.g. from 300 to $400 \mathrm{~K}$ ). The number of $\mathrm{HCO}_{3}{ }^{-}$participating in the 
reaction is $1 \%$, i.e., $n_{\mathrm{HCO}_{3}}^{\prime}=0.01 \times n_{\mathrm{HCO}_{3}}$, because spark discharge is assumed to heat only $1 \%$ of the total water volume. Then, the number of $\mathrm{OH}^{-}$one can produce for $\mathrm{E}_{\mathrm{a}} \approx 1 \mathrm{eV}$ becomes:

$$
n_{\mathrm{OH}^{-}}^{\prime}=n_{\mathrm{HCO}_{3}}^{\prime} \times k^{\prime}=n_{\mathrm{HCO}_{3}}^{\prime} \times A e^{-E_{a} / T^{\prime}}=0.01 \mathrm{An}_{\mathrm{HCO}_{3}} e^{-11000 / 400}=0.01 e^{-27.5} \mathrm{An}_{\mathrm{HCO}_{3}}=e^{-32} \mathrm{An}_{\mathrm{HCO}_{3}}
$$

Comparing the number of the hydroxyl ions produced for the two cases, i.e., $n_{\mathrm{OH}^{-}}$and $n_{\mathrm{OH}^{-}}^{\prime}$, one can see that the local heating by spark discharge can produce about 100 times more $\mathrm{OH}^{-}$, thus 100 times more efficiently precipitate dissolved calcium ions in hard water than the volume heating.

In conclusion, it has been demonstrated earlier that pulsed plasma discharge can trigger the precipitation process of calcium carbonate in hard water (Yang et al., 2010a). Equilibrium of calcium ion concentration after approximately 10-min plasma treatment was observed in previous experiments. The possibility to shift the equilibrium and enhance the precipitation was demonstrated by degassing the dissolved $\mathrm{CO}_{2}$ from water through spray circulation. It was hypothesized that the precipitation process was associated with different effects produced by the discharge. Experiments were conducted showing that UV radiation or reactive species produced by the spark discharge was negligible for the precipitation process. The effect of micro-heating was tested using a hot wire method, while the non-thermal effect of the plasma was investigated by application of a nanosecond pulsed electric discharge in water.

It was observed that both cases showed about $10 \%$ drop of calcium ion concentration, indicating that the precipitation process may be associated by both the thermal and non-thermal effect of by plasma in water. The morphology of the calcium carbonate particles collected from the two experiments was in agreement with that collected from water samples treated by conventional thermal spark discharge, indicating that the precipitation process may be associated by both the thermal and non-thermal effect of plasma. Further investigations are necessary to determine the detailed pathways of calcium carbonate precipitation by the pulsed plasma treatment.

\subsection{Economic Analysis on Plasma Water Treatment}

Finally, the anticipated benefits of the present plasma water treatment using spark discharges are discussed from the economic point of view. For a modern 1000-MW fossilfueled power plant with $40 \%$ efficiency, it would reject $1500 \mathrm{MW}$ of heat at the full load, and use about $2,800 \mathrm{~m}^{3} / \mathrm{min}$ of circulating water based on $10^{\circ} \mathrm{C}$ temperature difference in condenser (El-Wakil 1984). As heat is removed via evaporation of pure water at a cooling tower, the need for the makeup water is about $28 \mathrm{~m}^{3} / \mathrm{min}$ to compensate the loss through evaporation, wind drift and blowdown (El-Wakil, 1984). Assuming that the makeup water is moderately hard with a 
$\mathrm{CaCO}_{3}$ hardness of $100 \mathrm{mg} / \mathrm{L}$, the total amount of $\mathrm{CaCO}_{3}$ that the makeup water brings into the cooling tower becomes:

$$
\dot{\mathrm{m}}_{\mathrm{CaCO}_{3}}=28 \mathrm{~m}^{3} / \mathrm{min} \times 100 \mathrm{mg} / \mathrm{L}=2800 \mathrm{~g} / \mathrm{min}
$$

In a typical cooling tower application, the cycle of concentration (COC) is usually maintained at 3.5. That means the hardness in the circulating cooling water is maintained at approximately $350 \mathrm{mg} / \mathrm{L}$, and the blowdown rate can be calculated if the wind drift loss is neglected:

$$
\mathrm{q}_{\text {blowdown }}=\frac{\mathrm{q}_{\text {makeup }}}{\operatorname{COC}}=8 \mathrm{~m}^{3} / \mathrm{min}
$$

It has been demonstrated that the present pulsed plasma technology could continuously precipitate $\mathrm{Ca}^{2+}$ from water, and potentially allows a cooling tower to operate at a higher COC than the current standard due to the reduction in blowdown frequency. Ideally, zero blowdown could be achieved, if all the $\mathrm{Ca}^{2+}$ brought in by the makeup water is precipitated by the plasma, thus the constant mineral concentration is maintained in the main cooling loop without blowdown. The energy cost, as shown earlier, is about $1800 \mathrm{~J} / \mathrm{L}$ to achieve in average $25 \%$ reduction in water hardness. Assuming that the cooling water is treated in a side-stream loop, the flow rate needed becomes:

$$
\mathrm{q}=\frac{\dot{\mathrm{m}}_{\mathrm{CaCO}_{3}}}{350 \mathrm{mg} / \mathrm{L} \times 25 \%}=32 \mathrm{~m}^{3} / \mathrm{min}
$$

which is approximately $1.2 \%$ of the flow rate of the main loop. The power needed to treat the water in the side-stream loop can be calculated as:

$$
\mathrm{P}=\frac{1800 \mathrm{~J} / \mathrm{L} \times 32 \times 10^{3} \mathrm{~L} / \mathrm{min}}{60 \mathrm{~s} / \mathrm{min}}=960 \mathrm{~kW}
$$

which is $0.1 \%$ of the full capacity of the $1000-\mathrm{MW}$ power plant. In the mean while, the flow rate of the makeup water can be reduced from 28 to $20 \mathrm{~m}^{3} / \mathrm{min}$, which is equal to a saving of approximately $11,500 \mathrm{~m}^{3}$ per day, due to the elimination of blowdown.

\subsection{Application for Mineral Fouling Mitigation in Heat Exchangers}

Calcium carbonate is the most common scale-forming mineral occurring in industrial water facilities. It is generally the first mineral to precipitate out either by heating or by concentrating water due to its relatively low solubility, although its concentration in source water significantly varies depending on locations. Control of calcium carbonate scale is thus 
often the limiting factor in most industrial cooling water applications, as it decreases the efficiency of heat exchangers because of the insulating effect of the deposits. Furthermore, the formed deposits reduce the opening area in heat exchanger tubes, thus requiring more pumping power if one desires to maintain a constant flow rate (Panchal and Knudsen, 1998; Somerscales, 1990; Cho et al., 2003; Cho et al., 2005). A 0.8-mm layer of $\mathrm{CaCO}_{3}$ scale can increase the energy use by about 10\% (The US Department of Energy, 1998). If one can prevent or mitigate fouling on heat transfer surfaces, it not only increases heat exchanger efficiency, but also reduces the expenses associated with the cleaning of fouled heat exchangers. In addition to the benefit of reduced mineral fouling, the COC can be increased, resulting in water savings by reduced make-up and blowdown as shown in the previous section and other previous studies (Muller-Steinhagen, 2000; Radler and Ousko-Oberhoffer, 2005; Tijing et al., 2009; Demadis et al., 2007). Blowdown is the water drained from cooling equipment to remove mineral build-up in the circulating water.

Various chemical and non-chemical methods have been used to prevent the mineral fouling. Among them the scale-inhibiting chemicals like brominated compounds were the best choices, as they had a relatively high success rate. Due to safety issue and environmental concerns with the chemicals, there is a need for a new approach which is safe and clean from both environmental and cost points of view in cleaning and maintenance of heat exchangers. Physical water treatment (PWT) is a non-chemical method to mitigate mineral fouling as described earlier. Numerous studies have been reported for the effectiveness of ultrasonic, solenoid coils, magnetic fields, catalytic material, and electrolysis. Recently Yang and his coworkers (Yang et al., 2010a) reported that oversaturated hard water treated by underwater pulsed spark discharge may induce the precipitation of calcium carbonate in supersaturated water and produce a significantly greater number of particles than the untreated water. Note that the precipitation of dissolved mineral ions takes place in the bulk water instead of on the heat exchanger surfaces. This is the key process for all PWT methods, as the particles suspended in water tend to form a soft coating on heat transfer surfaces. If the shear force produced by flow is large enough to remove the soft coating, mineral fouling can be prevented or mitigated.

In the present study, direct pulsed spark discharge generated in water is used to mitigate mineral fouling in a double-pipe heat exchanger. The new method of using microsecondduration pulsed plasma in water is a major improvement over the aforementioned PWT because the previous PWT produce induced electric fields in water, where the field strength is often very small $(\sim 1 \mathrm{mV} / \mathrm{cm})$ due to involved physics laws such as Faraday's law. In comparison, an electric field above $10^{6} \mathrm{~V} / \mathrm{cm}$ could be produced by pulsed plasma in water in the present study, leading to higher efficiency than the previous PWT devices. 


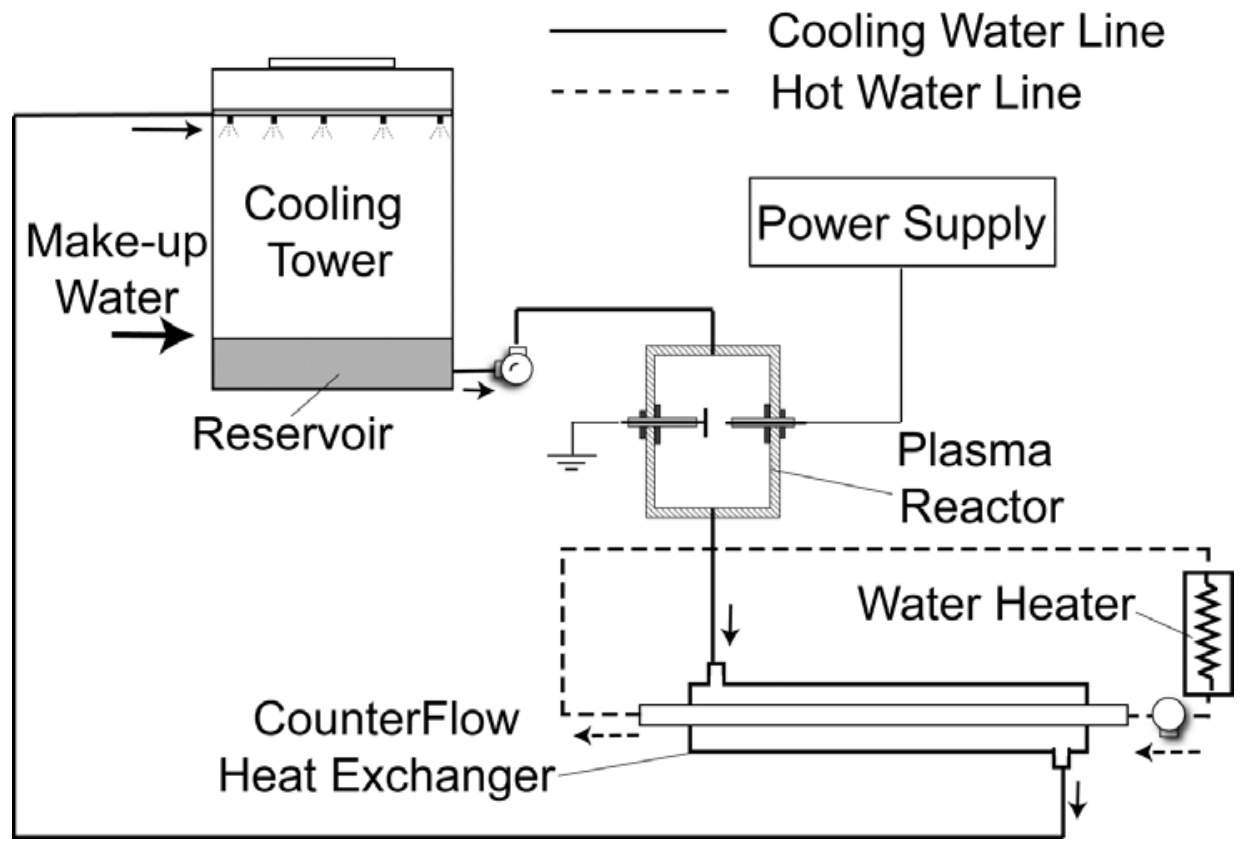

Figure 6-25. Schematic diagram of the experimental set-up. (Yang et al., 2010b).

The present study conducted fouling experiments in a heat exchanger by circulating artificially-prepared hard water through a simulated cooling tower system. Figure 6-25 shows the schematic diagram of the present test facility, which consisted of two separate loops for circulation of hot and cooling water, a pulsed spark discharge generation system, a cooling tower, a heat exchanger test section, pumps and a data acquisition system.

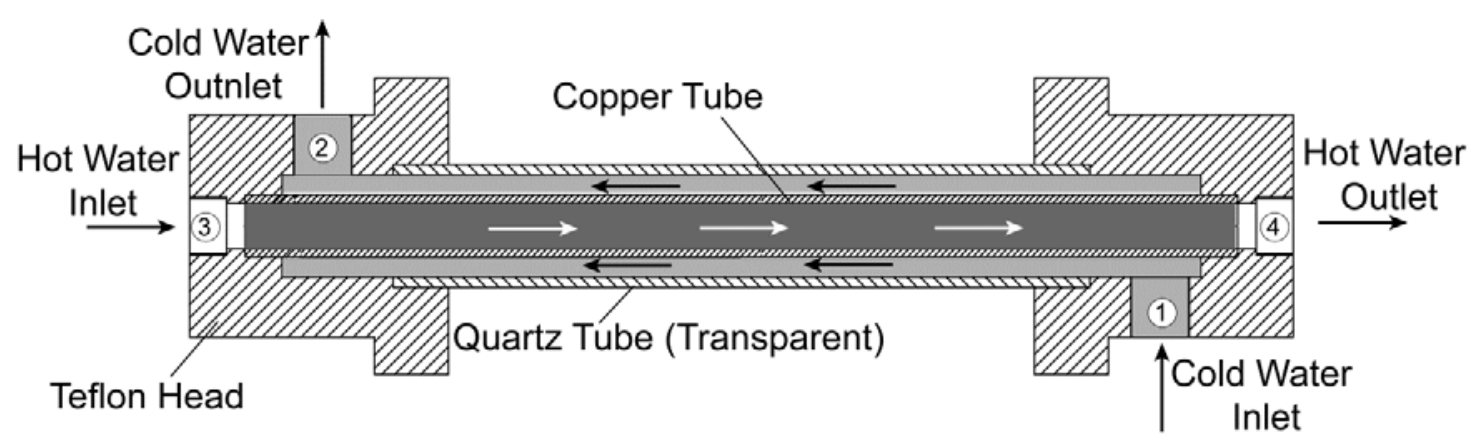

(1) (2) (3) (4): Thermal Couples

Figure 6-26. Schematic diagram of the heat transfer test section (Yang et al., 2010b).

Figure 6-26 shows the schematic diagram of the counterflow concentric type heat exchanger. The heat transfer rate, $\mathrm{Q}$, was calculated from both hot- and cooling-water sides as: 


$$
\mathrm{Q}=\dot{\mathrm{m}}_{\mathrm{h}} \mathrm{c}_{\mathrm{p}} \Delta \mathrm{T}_{\mathrm{h}}=\dot{\mathrm{m}}_{\mathrm{c}} \mathrm{c}_{\mathrm{p}} \Delta \mathrm{T}_{\mathrm{c}}
$$

where $\dot{\mathrm{m}}_{\mathrm{h}}$ and $\dot{\mathrm{m}}_{\mathrm{c}}$ are the mass flow rates of hot and cooling water, respectively; $\mathrm{c}_{\mathrm{p}}$ is the specific heat of water; $\Delta \mathrm{T}_{\mathrm{h}}$ and $\Delta \mathrm{T}_{\mathrm{c}}$ are the temperature differences between inlet and outlet of hot and cooling water, respectively. The heat transfer rates at hot and cooling water sides should be equal to each other under ideal conditions. In reality, the heat transfer rate in the hot water side was less, approximately $5 \%$, than that in the cold water side as parasitic heat loss takes place to the surroundings in spite of insulation. Hence, the heat transfer rate measured from the cooling water side was used to calculate the overall heat transfer coefficient. The heat transfer rate $\mathrm{Q}$ varied from 1.9 to $3.2 \mathrm{~kW}$ depending on the flow velocity at the cold-water side.

The overall heat transfer coefficient $U$ was calculated using the following equation (Incropera et al., 2007):

$$
U=\frac{Q_{c}}{A_{o} \Delta T_{L M T D}}
$$

The heat transfer surface area $A_{o}$ was calculated using the outer diameter of the copper tube $\left(d_{o}\right.$ $=22.2 \mathrm{~mm}$ ) with an effective heat transfer length of $600 \mathrm{~mm}$ (i.e., $\mathrm{A}_{\mathrm{o}}=\pi \mathrm{d}_{\mathrm{o}} \mathrm{L}_{\text {effective}}$ ). The logmean-temperature-difference, $\Delta \mathrm{T}_{\mathrm{LMTD}}$, was determined as follows (Incropera et al., 2007):

$$
\Delta T_{\text {LMTD }}=\frac{\left(T_{h, o}-T_{c, i}\right)-\left(T_{h, i}-T_{c, o}\right)}{\ln \left[\frac{\left(T_{h, o}-T_{c, i}\right)}{\left(T_{h, i}-T_{c, o}\right)}\right]}
$$

The fouling resistance, $R_{f}$ was calculated using the following equation (Incropera et al., 2007):

$$
R_{f}=\frac{1}{U_{f}}-\frac{1}{U_{i}}
$$

where $U_{f}$ is the overall heat transfer coefficient for fouled states, while $U_{i}$ is the overall heat transfer coefficient corresponding to the initial clean state. The latter $\left(U_{i}\right)$ was determined using distilled water (without chemicals) and without the use of PWT device during the initial calibration run prior to the fouling tests with artificial hard water. 


\subsubsection{Fouling Resistance: Validation Study}

Figure 6-27 shows the results for the fouling tests obtained using water hardness of 250 ppm for the no-treatment and plasma treated cases at a flow velocity of $0.1 \mathrm{~m} / \mathrm{s}$. Due to high water hardness, there was no induction period in both cases. An induction period is usually depicted by a straight horizontal line in the beginning of the fouling curve, which indicates lateral spreading of scale deposits on heat transfer surface. In the present study, the artificial hard water that contained calcium and bicarbonate ions reacted quickly to the hot heat transfer surface, making immediate depositions of calcium salt particles on the surface as soon as the fouling test begun.

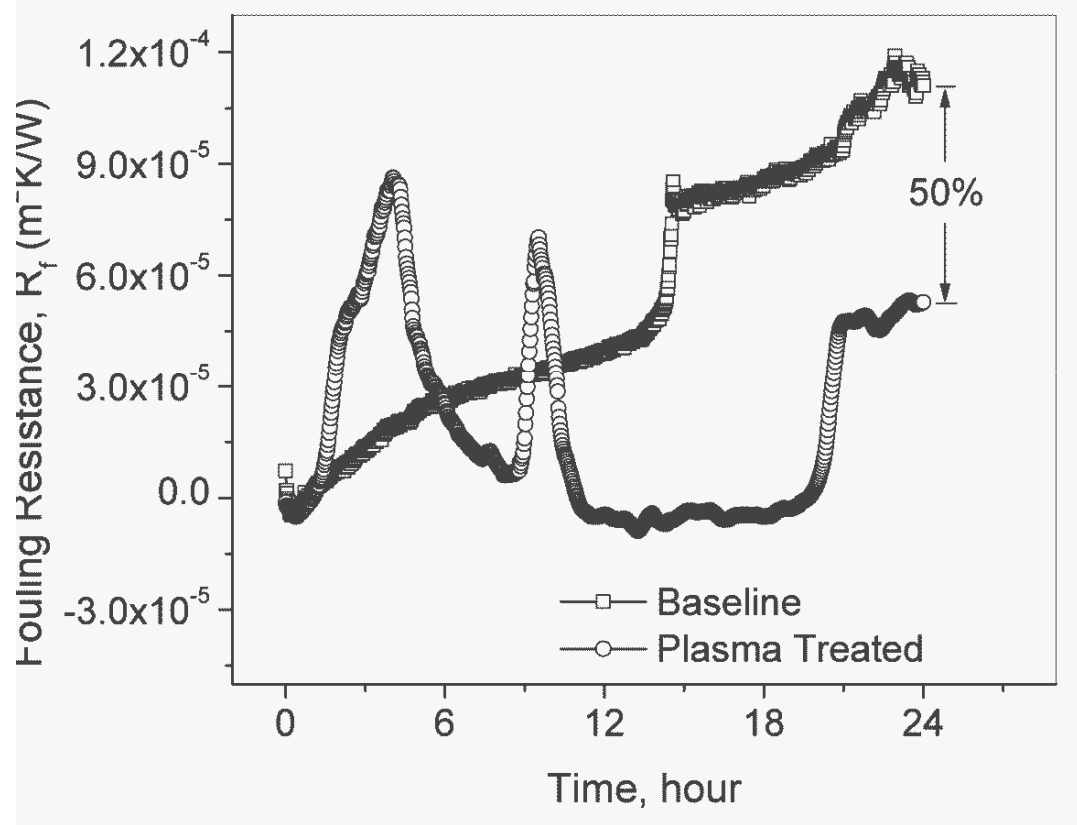

Figure 6-27. Fouling resistances for $250 \mathrm{ppm}$ hard water under no-treatment and plasma treated cases with a flow velocity of $0.1 \mathrm{~m} / \mathrm{s}$ (Yang et al., 2010b).

The scale deposition involved the cumulative effect of a direct diffusion of dissolved calcium ions to the heat transfer surface and the deposition of precipitated calcium salt particles due to supersaturated conditions and accelerated precipitation of calcium salts by PWT. The fouling resistance in the no-treatment case demonstrated a slow increase in the first $14 \mathrm{~h}$ of operation. At $\mathrm{t}=15 \mathrm{~h}$, the fouling resistance increased dramatically as the entire surface of 
copper tube was fully covered by mineral scales. After $t=16 \mathrm{~h}$, the fouling resistance began to rise as the thickness of the scale layer slowly increased until the end of test, indicating that the deposition rate of the scales was consistently larger than the removal rate during this period because of the slow flow velocity.

The fouling resistance curves obtained in the cases for the plasma treatment depicted a completely different trend compared to that obtained for the no-treatment case. The fouling resistance had a steep increase to a maximum value in the first $4 \mathrm{~h}$ of operation. Note that there are two different categories of fouling: particulate fouling and precipitation fouling. The former refers to the adhesion of suspended particles to the heat transfer surface in the form of soft sludge. This type of fouling can easily be removed by shear forces created by flow than those deposits produced from the precipitation of mineral ions directly on the solid heat transfer surface, i.e., precipitation fouling. It was demonstrated in the authors' previous study that the precipitation of calcium carbonate could be induced by application of pulsed spark discharge in supersaturated hard water and thus creating a significantly greater number of $\mathrm{CaCO}_{3}$ particles than the untreated water. Hence, much faster particulate fouling took place at the first several hours of the test, causing the dramatic increase in the fouling resistance. At $t=4 \mathrm{~h}$, the fouling resistance showed a significant drop, indicating that large scale pieces was dislodged due to the shear stress of the water flow. The similar particulate fouling build-up and dislodge process were repeated during the period between 9 and $12 \mathrm{~h}$. The final asymptotic fouling resistance at the end of the test was 50\% lower than that obtained from baseline test, clearly indicating the beneficial effect of the plasma discharge on the mitigation of mineral fouling.

Figure 6-28 shows photographs of sections of fouled copper tubes for the no-treatment and plasma treated cases, which were taken after the copper tubes were removed from the heat transfer test section and completely dried. Visual inspection on the fouled tubes indicated that there were thick scale deposits $(>1 \mathrm{~mm})$ over the entire tube surface for the no-treatment case. For the case of the plasma treatment, the scale deposits appeared to be much thinner than that observed in the no-treatment case. One could clearly see the copper-tone color of the tube at the end of fouling test for the plasma treated case, indicating that the pulsed spark discharge could significantly mitigate the scale deposits on the tube surface. 


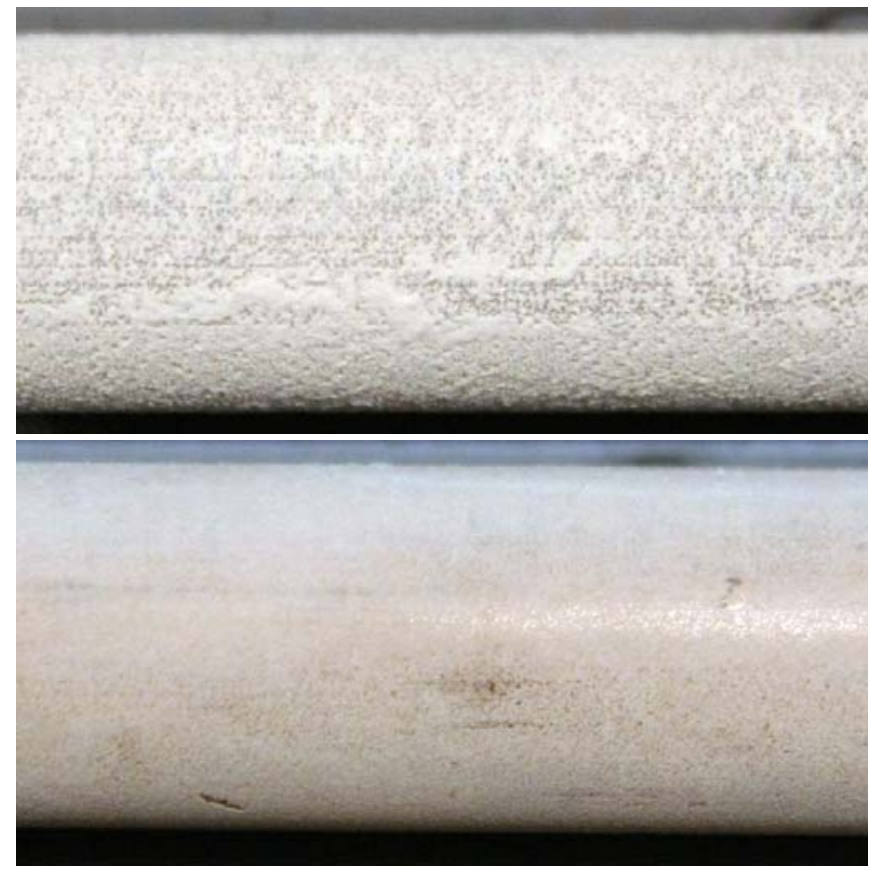

Figure 6-28. Photographic images of the scales for (a) no-treatment; (b) plasma treatment cases $\left(\mathrm{CaCO}_{3}\right.$ hardness of $250 \mathrm{ppm}$ and a flow velocity of $\left.0.1 \mathrm{~m} / \mathrm{s}\right)$ (Yang et al., 2010b).

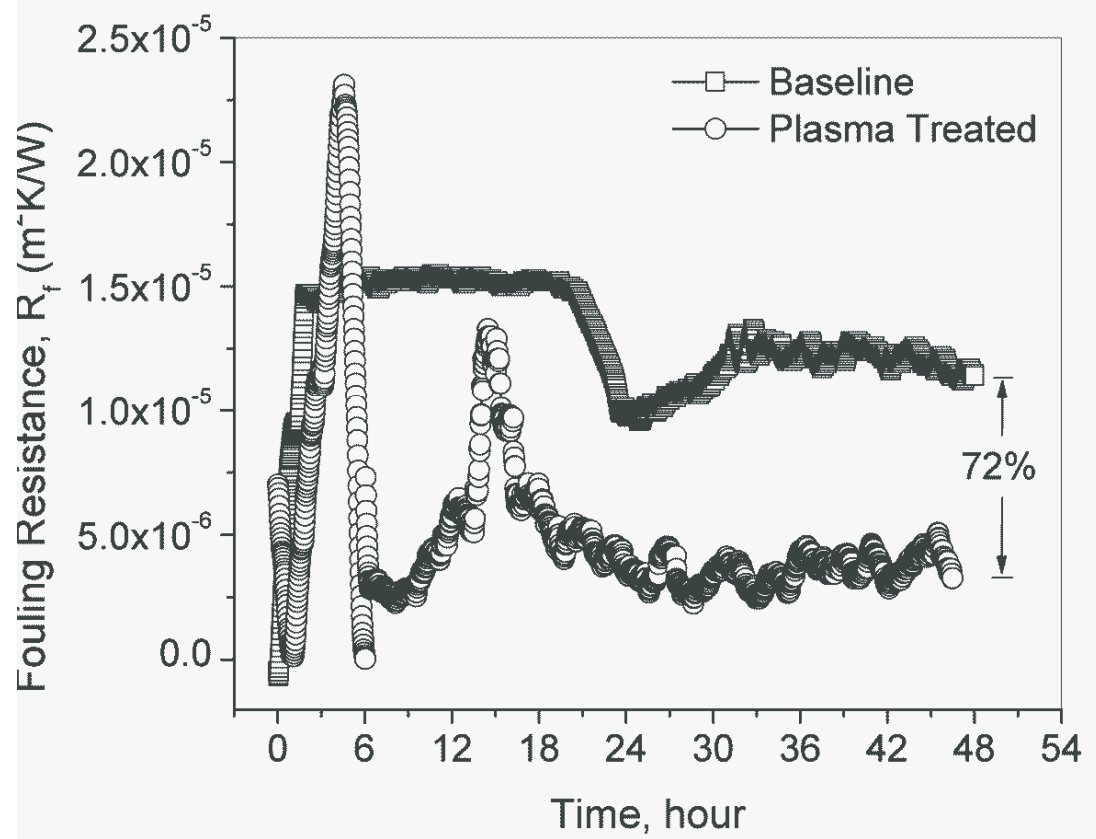

Figure 6-29. Fouling resistances for $250 \mathrm{ppm}$ hard water under no-treatment and plasma treated cases with a flow velocity of $0.5 \mathrm{~m} / \mathrm{s}$ (Yang et al., 2010b). 
Figure 6-29 shows the results for the fouling tests obtained using water hardness of 250 ppm for both cases at a flow velocity of $0.5 \mathrm{~m} / \mathrm{s}$ with zero blowdown. The overall fouling resistance showed a significant drop comparing with that obtained at $0.1 \mathrm{~m} / \mathrm{s}$, mainly because of the higher removal rate caused by a higher flow velocity. For the no-treatment case, the fouling resistance had a steep increase to a local maximum value in the first $3 \mathrm{~h}$ of operation. At $\mathrm{t}=3 \mathrm{~h}$, the fouling resistance stopped increasing and the value remained unchanged for the next $16 \mathrm{~h}$, indicating that there must have been some balance between the deposition rate and removal rate for the no-treatment case due to the higher shear stress produced by a high flow velocity. At $t=$ $19 \mathrm{~h}$, the no-treatment case showed a significant drop in the fouling resistance. After $\mathrm{t}=30 \mathrm{~h}$, the fouling resistance showed slight up-and-down trends with its mean value slightly decreasing with time till the end of the test.

The fouling resistance curves obtained in the case for plasma treatment at $0.5 \mathrm{~m} / \mathrm{s}$ depicted similar trend as the $0.1 \mathrm{~m} / \mathrm{s}$ case shown in Figure 6-29 at the first $20 \mathrm{~h}$ period, although at a much lower value. The fast rise and fall of fouling resistance observed in the first 6 -h period indicated both accumulation and removal of particulate fouling on the heat exchanger surface. After $\mathrm{t}=20 \mathrm{~h}$, the fouling resistances slightly went up and down numerous times until the end of the test. The up-and-down trends of the fouling resistance clearly indicated that the old scales were repeatedly removed from the heat transfer surface as the new scales continued to develop. The surface was not fully covered by scales at the end of the test, and the final fouling resistance was reduced by $72 \%$ comparing to that for the no-treatment case. The improved efficiency in mitigating fouling observed in the plasma treated case can be explained as follows: calcium ions were continuously precipitated to calcium salt particles by the spark discharge. Subsequently particulate fouling took place as calcium particles adhered to the heat transfer surface, creating a soft sludge coating on the surface. The coating can be more easily removed due to high flow. Similar results were reported previously with a solenoid coil by Cho et al. (Cho et al., 2004; Cho et al., 2003; Cho et al., 2005), who showed better mitigation results at a high flow rate in the study. 

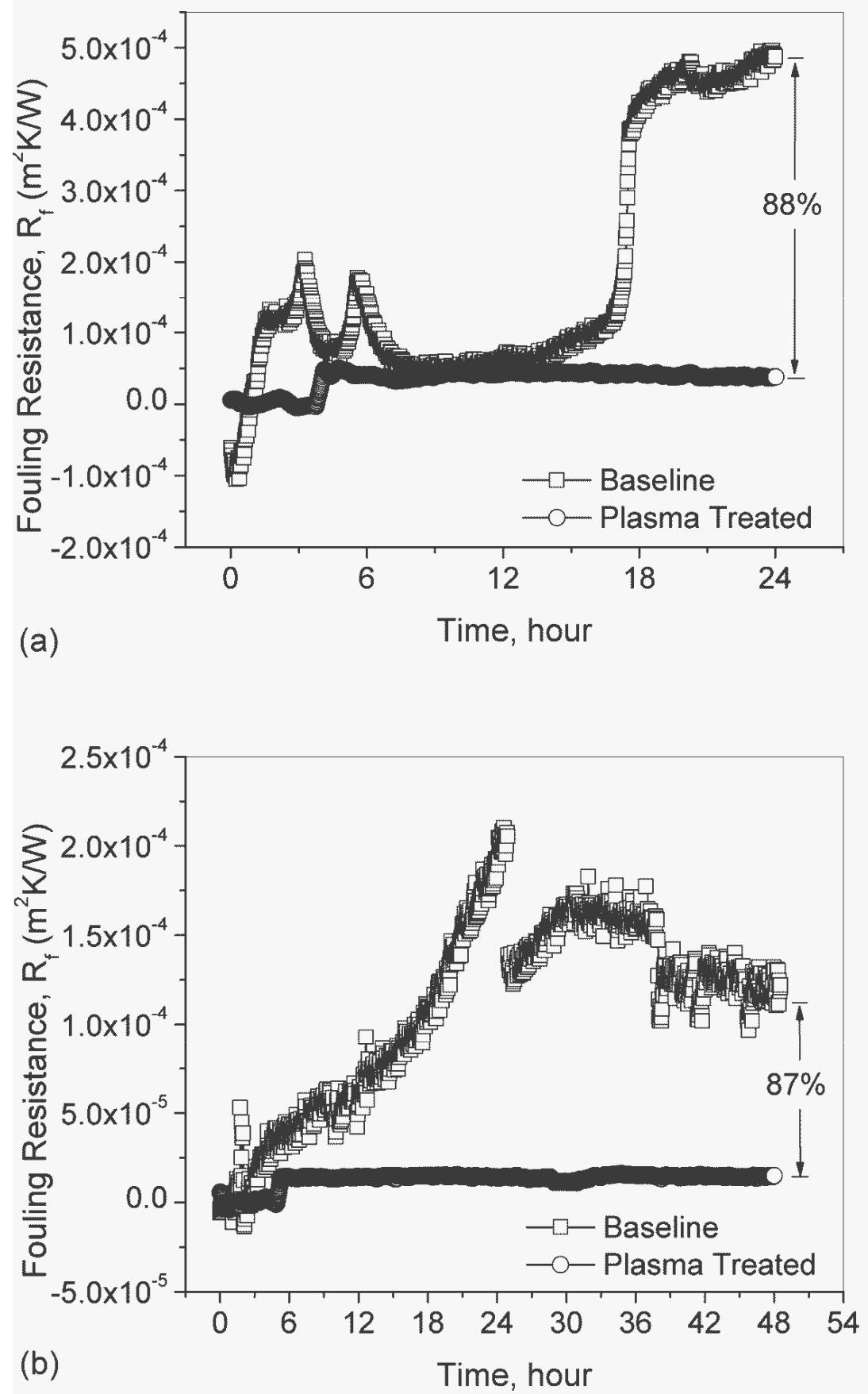

Figure 6-30. Fouling resistances for $500 \mathrm{ppm}$ hard water under no-treatment and plasma treatment cases with two different flow velocities: (a) $0.1 \mathrm{~m} / \mathrm{s}$; (b) $0.5 \mathrm{~m} / \mathrm{s}$ (Yang et al., 2010b). 
Figure 30(a) and (b) show the results for the fouling tests obtained for the case of water hardness of $500 \mathrm{ppm}$ and flow velocities of 0.1 and $0.5 \mathrm{~m} / \mathrm{s}$ for the plasma treated and notreatment cases. Due to the high water hardness used in the present study, the rate of increase in the fouling resistance was very steep for the no-treatment case at $0.1 \mathrm{~m} / \mathrm{s}$, which reached the local maximum at $\mathrm{t}=3 \mathrm{~h}$. After this point, the fouling resistance significantly decreased till $\mathrm{t}=5$ $\mathrm{h}$, indicating that the removal of scale particles was greater than the new deposits during this period, probably due to a reduced opening in the heat transfer test section by the scale deposits and subsequently increased wall shear stress. Again, the similar cycle repeated during 5-9 $\mathrm{h}$ period. Note that the supersaturation level in the cooling water was extremely high so that there must have been a large number of suspended $\mathrm{CaCO}_{3}$ particles in water even in the no-treatment case, leading to particulate fouling on the heat transfer surface. Thus, one might expect that the scale deposits might have been soft, which helped increasing the removal rate.

For the cases of plasma treatment, there was an induction period of approximately $3 \mathrm{~h}$ before a sudden but brief increase of fouling resistance, indicating that the heater exchanger surface was fully covered by the scales at $\mathrm{t} \approx 3 \mathrm{~h}$. After $\mathrm{t} \approx 4 \mathrm{~h}$ the fouling resistance impressively remained constant till the end of the test. The asymptotic value for the plasma treated case was about $88 \%$ lower than that for the no-treatment case.

Figure 6-30(b) presents the fouling resistance for the flow velocity of $0.5 \mathrm{~m} / \mathrm{s}$, showing that there was also no induction period for the no-treatment case. As the velocity of cold water in the heat transfer test section was increased from 0.1 to $0.5 \mathrm{~m} / \mathrm{s}$, there was less fouling deposit in general as the removal rate increased due to increased shear force, a phenomenon which was also reported by a recent study (Yang et al., 2009). Note that at a high velocity, there was a high mass deposition rate. However, the shear force created by the flow increased such that the scales are more efficiently removed, resulting in reduced fouling resistances. An $87 \%$ drop in the fouling resistance was obtained for the plasma treated case compared with the no-treatment case, again confirming the effectiveness of the plasma treatment of water on mitigating the mineral fouling. It is of note that even for the case of $500 \mathrm{ppm}$, where the hardness became about $1500 \mathrm{ppm}$ near the end of test with zero blowdown, the plasma treated case could reduce the fouling resistance by 88 and $87 \%$ for flow velocities of 0.1 and $0.5 \mathrm{~m} / \mathrm{s}$, respectively.

\subsubsection{Visualization of the Calcium Carbonate Particles}

Figures 6-31(a) and (b) show SEM images of $\mathrm{CaCO}_{3}$ scales for both the no-treatment and plasma treated cases for $250 \mathrm{ppm}$ hard water at a flow velocity of $0.5 \mathrm{~m} / \mathrm{s}$. The SEM images for the no-treatment case showed particles less than $10 \mu \mathrm{m}$ in size, with sharp and pointed tips in crystal structures, whereas those obtained with plasma treatment showed particles of $15 \mu \mathrm{m}$ in size and above, due to the precipitation effect of spark discharges, with blunt edged crystals. The sharp and pointed crystal tips observed in the no-treatment case are believed to be produced through precipitation reactions of mineral ions on the heat transfer surface, thus adhering to the heat transfer surface more strongly than blunt crystals observed in the plasma case. 

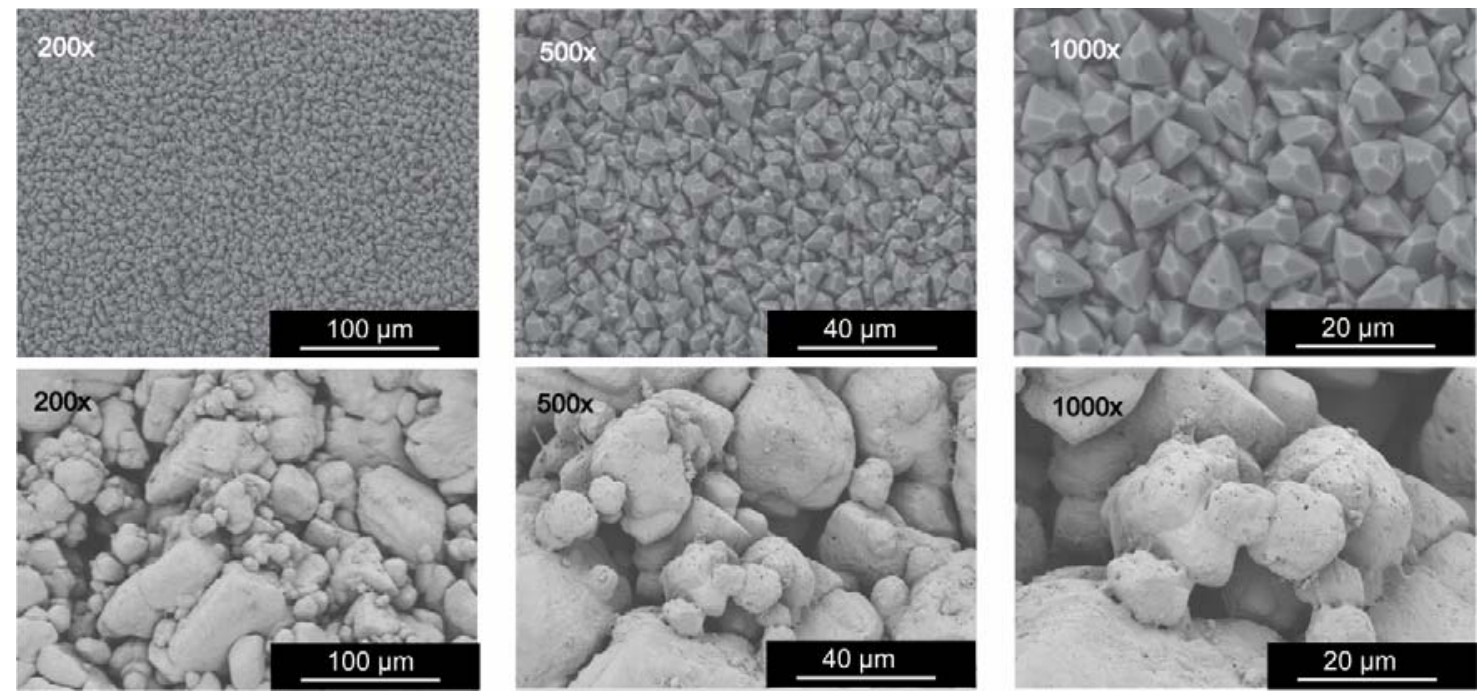

Figure 6-31. SEM photographs of the scales obtained for (top) no-treatment; (bottom) plasma treated cases $\left(\mathrm{CaCO}_{3}\right.$ hardness of $250 \mathrm{ppm}$ and a flow velocity of $0.5 \mathrm{~m} / \mathrm{s}$ ) (Yang et al., 2011c).

Calcium carbonate is a crystalline substance that exists in three polymorphs: calcite, aragonite and vaterite (Smith, 1986). Each polymorph has a unique crystallographic structure with a unique XRD spectrum that serves as its fingerprint. The present XRD analyses were conducted to determine the crystallographic phase of scale deposits so that the focus was on spectrum peaks and not on the intensity. Figure 34(a) shows the standard XRD spectra of the calcite phases of calcium carbonate as a reference, which has a prominent peak of intensity at $2 \theta$ $=29.5^{\circ}$. 


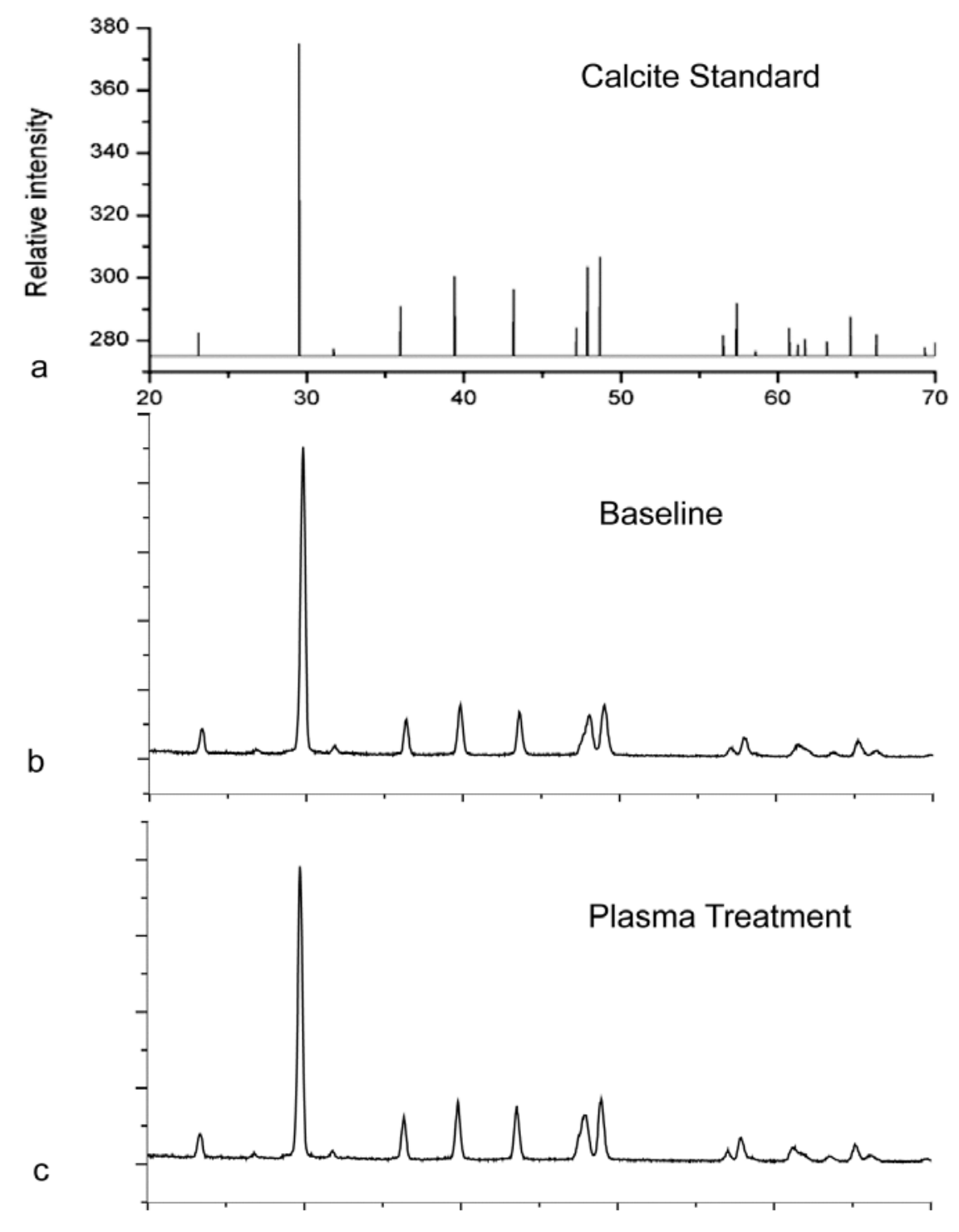

Figure 6-32. XRD analyses of the scales for (a) standard calcite; (b) no-treatment; (c) plasma treated cases $\left(500 \mathrm{ppm} \mathrm{CaCO}_{3}\right.$ hardness and a flow velocity of $0.1 \mathrm{~m} / \mathrm{s}$ ). (Yang et al., 2011c)

Figures 6-32 (b) and (c) presents the results of the XRD analyses for the no-treatment and plasma treated cases at water hardness of $500 \mathrm{ppm}$ and a flow velocity of $0.1 \mathrm{~m} / \mathrm{s}$. The results were compared to the standard XRD spectra of $\mathrm{CaCO}_{3}$ given in Figure 6-32(a). For both cases, the peaks depicted that of a calcite crystal. Although most previous studies reported aragonite crystals for the no treatment cases (Cho et al., 2004; Kontoyannis and Vagena, 1999), the present XRD result did not show aragonite for the no-treatment case. It can be attributed to the fact that even for the no-treatment cases there must have been a large number of suspended calcium particles in water in the present study due to extremely supersaturated states of cooling water and no blowdown in the study. Hence, even for the no-treatment case, one might expect 
to have a large number of suspended particles in water, thus leading to a calcite form of $\mathrm{CaCO}_{3}$ scales on the heat transfer surface.

For the cases of plasma treatment, spark discharge would produce more suspended calcium particles in hard water than the no-treatment case (Yang et al., 2010a), producing particulate fouling or calcite form of calcium crystal at the heat transfer surface. The XRD results for the no-treatment and plasma treated cases at water hardness of 250 and a flow velocity of $0.5 \mathrm{~m} / \mathrm{s}$ were similar to the results given in Figure 32 .

\subsubsection{Cycle of Concentration (COC)}

The COC is defined as the ratio of the dissolved solids in cooling-tower water to those in makeup supply water. Figure 33 shows variations in COC over time for the case of 250-ppm hard water with a flow velocity of $0.5 \mathrm{~m} / \mathrm{s}$. The value of COC increased almost linearly with time because of zero blowdown. The COC reached approximately 2.8 at the end of the fouling test for the no-treatment case, whereas it arrived at 2.4 for the plasma treated case. Since the hardness of the makeup water was $250 \mathrm{ppm}$, the hardness of circulating water became approximately $600-700 \mathrm{ppm}$ at the end of the fouling tests for both cases. In addition, the COC value for the plasma treated case was consistently smaller by about $0.3-0.5$ than those for the no-treatment cases during the entire fouling test, reflecting the fact that the pulsed spark discharge was continuously precipitating calcium ions from water. Note that the COC for other cases studied in the study (i.e. at different flow velocities and different hardness) also reached approximately 3 at the end of fouling tests with zero blowdown, indicating that the water hardness was about 750 and 1500 ppm for 250 and 500 ppm cases, respectively, near the end of the test. Such extremely harsh fouling conditions were utilized in the study in order to expedite the fouling process and examine the performance and limitation of the pulsed spark discharge system. 


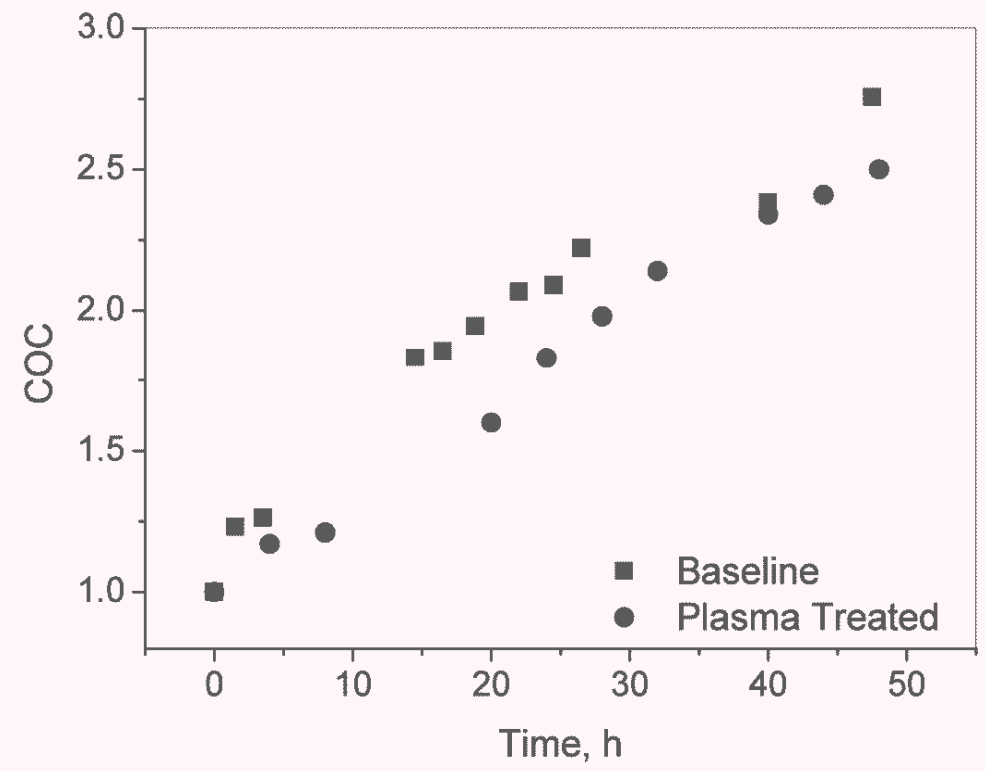

Figure 6-33. Variations in cycle of concentration (COC) vs. time for $250 \mathrm{ppm}$ hard water under no-treatment and plasma treated cases with a flow velocity of $0.5 \mathrm{~m} / \mathrm{s}$. (Yang et al., 2011c) 


\section{CONCLUSIONS AND RECOMMENDATION}

The hypothesis of the present study was that if we could successfully precipitate and remove the excess calcium ions in cooling water, we could prevent condenser-tube fouling and at the same time double the COC.

Hence, the overall objective of the present work was to utilize spark discharges to continuously precipitate and remove dissolved mineral ions (such as calcium and magnesium) in cooling water so that one could eliminate or significantly reduce blowdown.

The present study began with a basic scientific research to better understand the mechanism of pulse spark discharges in water and conducted a series of validation experiments using hard water in a laboratory cooling tower. Task 1 of the present work was to demonstrate if the spark discharge could precipitate the mineral ions in water. Task 2 was to demonstrate if the selfcleaning filter could continuously remove these precipitated calcium particles such that the blowdown could be eliminated or significantly reduced. Task 3 was to demonstrate if the scale could be prevented or minimized at condenser tubes with a COC of 8 or (almost) zero blowdown. In Task 1, we successfully completed the validation study that confirmed the precipitation of dissolved calcium ions in cooling water with the supporting data of calcium hardness over time as measured by a calcium ion probe. In Task 2 , we confirmed through experimental tests that the self-cleaning filter could continuously remove precipitated calcium particles in a simulated laboratory cooling tower such that the blowdown could be eliminated or significantly reduced. In addition, chemical water analysis data were obtained which were used to confirm the COC calculation. In Task 3, we conducted a series of heat transfer fouling tests using a condenser heat exchanger in the laboratory cooling tower, from which we confirmed that the plasma water treatment technology could prevent or significantly mitigate mineral foulings in condenser tubes when compared with the no-treatment case.

With the completion of the present work, a cooling water treatment technology using pulse spark discharges is currently ready for field-validation tests. Thus, it is recommended that a fieldvalidation test should be carried out. For the field-validation tests, the power supply for the generation of spark discharge should be scaled-up to a much bigger, i.e., at the order of $5 \mathrm{~kW}$. Furthermore, a system of multiple spark discharges produced by one large power supply should be developed using a high-voltage distributor for energy-efficient operation of the plasma water treatment technology.

The plasma water treatment technology is a true mechanical water softener with almost no maintenance, which continuously converts hard water to soft water spending a relatively small amount of energy. Such a mechanical water softener could find wide-spread applications to solve hard water problems both in industry and at home. 


\section{REFERENCES}

Adams, C., Timmons, T., Seitz, T., Lane, J. and Levotch, S. (2005) Trihalomethane and haloacetic acid disinfection by-products in full-scale drinking water systems. J. Envir. Engrg. 131, 526-534.

Akiyama, H. (2000) Streamer discharges in liquids and their applications. IEEE Trans. Dielectr. Electr. Insul. 7, 646-653.

Akiyama, H., Sakugawa, T. and Namihira, T. (2007) Industrial applications of pulsed power technology. IEEE Trans. Dielec. Dielectr. Electr. Insul. 14, 1051-1064.

Akiyama, H., Takashi Sakugawa, and Takao Namihira etc., Industrial Applications of Pulsed Power Technology, IEEE Transactions on Dielectrics and Electrical Insulation 14(5) 2007 pp 1051-1064

An, W., Baumung, K. and Bluhm, H. (2007) Underwater streamer propagation analyzed from detailed measurements of pressure release. J. Appl. Phys. 101, 053302.

Arif Malik, M., Abdul Ghaffar and Salman Akbar Malik, Water purification by electrical discharges, Plasma Sources Sci. Technol. 102001 pp82-91

Assael, M.J., Karagiannidis, L., Malamataris, N. and Wakeham, W.A. (1998) The transient hotwire technique: a numerical approach. Int. J. Thermophysics 19, 379-389.

Avrorin, A. (1980) Shock compressibility at $\sim 100$ Mbar. JETF Lett.

Avrorin, A. (2006) Physical investigations under nuclear tests. UFN.

Baker, J.S. and Judd, S.J. (1996) Magnetic amelioration of scale formation. Water Research 30, 247-260.

Beroual, A. (1993) Electronic and Gaseous Processes in the Prebreakdown Phenomena of Dielectric Liquids. J. Appl. Phys. 73, 4528-4533.

Beroual, A., Zahn, M. and Badent, A. (1998) Propagation and Structure of Streamers in liquid Dielectrics. IEEE Electr. Insul. Mag. 14, 6-14.

Billamboz, N., Grivet, M., Foley, S., Baldacchino, G. and Hubinois, J.-C. (2010) Radiolysis of the polyethylene/water system: Studies on the role of hydroxyl radical. Radiat. Phys. Chem. 79, 36-40.

Bluhm, H., Frey, W. and Giese, H. (2000) Application of pulsed HV discharges to material fragmentation and recycling. IEEE Trans. Dielec. Dielectr. Electr. Insul. 7, 625-636.

Bolorizadeh, M.A. and Rudd, M.E. (1986) Angular and energy dependence of cross sections for ejection of electrons from water vapor. I. 50-2000-eV electron impact. Phys. Rev. A 33, 880-887.

Bott, T.R. (1995) The fouling of heat exchangers, Elsevier Science, New York.

Bruggeman, P., Verreycken, T., González, M.Á., Walsh, J.L., Kong, M.G., Leys, C. and Schram, D.C. (2010) Optical emission spectroscopy as a diagnostic for plasmas in liquids: opportunities and pitfalls. Journal of Physics D: Applied Physics 43(12), 124005.

Bruggeman, P., Walsh, J., Schram, D., Leys, C. and Kong, M.G. (2009) Time dependent optical emission spectroscopy of sub-microsecond pulsed plasmas in air with water cathode.

Plasma Sources Science and Technology 18(4), 045023. 
Buxton, G.V. and Elliot, A.J. (1986) Rate constant for reaction of hydroxyl radicals with bicarbonate ions. Radiat. Phys. Chem. 27, 241-243.

Carman, R.J., Ward, B.J., Mildren, R.P. and Kane, D.M. (2003) High-pressure dielectric barrier discharge Xenon lamps generating short pulses of high-peak-power VUV radiation $(172 \mathrm{~nm})$ with high pulse-to-pulse reproducibility, San Francisco, CA.

Chalker, P.R., Bull, S.J. and Rickerby, D.S. (1991) A review of the methods for the evaluation of coating-substrate adhesion. Mater. Sci. Eng. A 140, 583-592.

Champion, C. (2003) Theoretical cross sections for electron collisions in water: structure of electron tracks. Phys. Med. Biol. 48, 2147-2168.

Chibowski, E., Holysz, L. and Wojcik, W. (1994) Changes in zeta potential and surface free energy of calcium carbonate due to exposure to radiofrequency electric field. Colloids Surfaces A: Physicochem. Eng. Aspects 92, 79-85.

Ching, W.K., Colussi, A.J., Sun, H.J., Nealson, K.H. and Hoffmann, M.R. (2001) Escherichia coli disinfection by electrohydraulic discharges. Environ. Sci.Technol. 35, 4139-4144.

Cho, Y.I., Fridman, A.F., Lee, S.H. and Kim, W.T. (2004) Physical water treatment for fouling prevention in heat exchangers. Advanced Heat Transfer 38, 1-72.

Cho, Y.I., Kim, W.T. and Cho, D.J. (2007) Electro-flocculation mechanism of physical water treatment for the mitigation of mineral fouling in heat exchangers. Experimental Heat Transfer 20, 323-335.

Cho, Y.I., Lane, J. and Kim, W. (2005) Pulsed-power treatment for physical water treatment. Int. Commun. Heat Mass Transfer 32, 861-871.

Cho, Y.I., Lee, S.H. and Kim, W.T. (2003) Physical water treatment for the mitigation of mineral fouling in cooling-water applications. ASHRAE Trans. 109, 346-357.

Choi, H.S., Shikovab, T.G., Titov, V.A. and Rybkin, V.V. (2006) Surface oxidation of polyethylene using an atmospheric pressure glow discharge with liquid electrolyte cathode. J. Colloid Interface Sci. 300, 640-647.

Chu, P.K., Tian, X.B., Wei, C.B., Yang, S.Q. and Fu, R.K.Y. (2006) Water plasma implantation/oxidation of magnesium alloys for corrosion resistance. Nuclear Instruments \&amp; Methods in Physics Research, Section B (Beam Interactions with Materials and Atoms) 242, 300-302.

Coetzee, P.P., Yacoby, M., Howell, S. and Mubenga, S. (1998) Scale reduction and scale modification effects induced by $\mathrm{Zn}$ and other metal species in physical water treatment. Water SA 24, 77-84.

Crittenden, J.C., Hu, S., Hand, D.W. and Green, S.A. (1999) A kinetic model for H2O2/UV process in a completely mixed batch reactor. Water Res. 33, 2315-2328.

Czernichowski, A. (1996) Gliding arc: Applications to engineering and environment control. Pure Appl. Chem. 66, 1301-1310.

De Baerdemaeker, F., Monte, M. and Leys, C. (2005) Capillary underwater discharges. IEEE Trans. Plasma. Sci. 33, 492-493. 
De Baerdemaeker, F., Simek, M. and Leys, C. (2007a) Efficiency of hydrogen peroxide production by ac capillary discharge in water solution. J. Phys. D: Appl. Phys. 40, 28012809.

De Baerdemaeker, F., Simek, M., Schmidt, J. and Leys, C. (2007b) Characteristics of ac capillary discharge produced in electrically conductive water solution. Plasma Sources Sci. Technol. 16, 341-354.

Demadis, K.D., Mavredaki, E., Stathoulopoulou, A., Neofotistou, E. and Mantzaridis, C. (2007) Industrial water systems: problems, challenges and solutions for the process industries. Desalination 213, 38-46.

Denisov, E.T., Sarkisov, O.M. and Likhtenshtein, G.I. (2003) Chemical Kinetics Fundamentals and New Developments, Elsevier, Amsterdam.

Derakhshesh, M., Abedi, J. and Hassanzadeh, H. (2010) Mechanism of methanol decomposition by non-thermal plasma. J. Electrostat. 68, 424-428.

Destaillats, H., Lesko, T.M., Knowlton, M., Wallac, H. and Hoffmann, M.R. (2001) Scale-up of sonochemical reactors for water treatment. Ind. Eng. Chem. Res. 40, 3855-3860.

Dhanasekaran, R. and Ramasamy, P. (1986) Two-dimensional nucleation in the presence of an electric field. J. Crystal Growth 79, 993-996.

Dingfelder, M., Hantke, D., Inokuti, M. and Paretzke, H.G. (1999) Electron inelastic-scattering cross sections in liquid water. Radiation Physics and Chemistry 53(1), 1-18.

Djuric, N.L., Cadez, I.M. and Kurepa, M.V. (1988) H2O and D2O total ionization crosssections by electron impact. International Journal of Mass Spectrometry and Ion Processes 83(3), R7-R10.

Donaldson, J. and Grimes, S. (1988) Lifting the scales from our pipes. New Scientist 18, 43-46.

Dragsund, E., Andersen, A.B. and Johannessen, B.O. (2001) BallastWater Treatment by Ozonation, IMO, London.

Eggers, J. (1997) Nonlinear dynamics and breakup of free-surface flows. Rev. Mod. Phys. 69, 865-930.

El-Wakil, M.M. (1984) Powerplant technology, McGraw-Hill, New York.

El-Wakil, M.M., Power plant technology McGraw Hill, New York, 1984, p.268, pp. 732-734

Fathi, A., Mohamed, T., Gabrielli, C., Maurin, G. and Mohamed, B.A. (2006) Effect of a magnetic water treatment on homogeneous and heterogeneous precipitation of calcium carbonate. Water Research 40, 1941-1950.

Feeley, T.J. (2008) DOE/Office of Fossil Energy's Energy \& Water R\&D Program.

Feeley, T.J., Skone, T.J., Stiegel Jr, G.J., McNemar, A., Nemeth, M., Schimmoller, B., Murphy, J.T. and Manfredo, L. (2008) Water: A critical resource in the thermoelectric power industry. Energy 33(1), 1-11.

Fridman, A. (2008) Plasma Chemistry, Cambridge University Press, Cambridge.

Fridman, A. and Kennedy, L. (2006) Plasma Physics and Engineering, Taylor \& Francis Group, New York. 
Fridman, A., Gutsol, A. and Cho, Y.I. (2007) Non-thermal atmospheric pressure plasma. Advances in Heat Transfer 40, 1-134.

Fridman, G., Peddinghaus, M., Ayan, H., Fridman, A., Balasubramanian, M., Gutsol, A., Brooks, A. and Friedman, G. (2006) Blood coagulation and living tissue sterilization by floating electrode dielectric barrier discharge in air. Plasma Chemistry and Plasma Processing 26, 425-442.

Gabrielli, C., Maurin, G., Francy-Chausson, H., Thery, P., Tran, T.T.M. and Tlili, M. (2006) Electrochemical water softening: principle and application. Desalination 206, 150-163.

Gehr, R., Zhai, Z.A., Finch, J.A. and Rao, S.R. (1995) Reduction of soluble mineral concentration in CaSO4 saturated water using a magnetic field. Water Research 29, 933940.

Gidalevich, E. and Boxman, R. (2006) Sub- and supersonic expansion of an arc channel in liquid. J. Phys. D: Appl. Phys. 39, 652-659.

Gordillo-Vázquez, F.J. (2008) Air plasma kinetics under the influence of sprites. J. Phys. D: Appl. Phys. 41, 234016.

Grahl, T. and Markl, H. (1996) Killing of microorganisms by pulsed electric fields. Appl. Microbiol. Biotechnol 45, 148-157.

Hagelaar, G. (2008) BOLSIG+ Solver. LAPLACE.

He, F. and Hopke, P.K. (1993) Experimental study of ion-induced nucleation by radon decay. The Journal of Chemical Physics 99(12), 9972-9978.

Heesch, E.J.M., Pemen, A.J.M., Huijbrechts, A.H.J., van der Laan, P.C.T., Ptssinski, K.J., Zanstra, G.J. and de Jong, P. (2000) A fast pulsed power source applied to treatment of conducting liquids and air. IEEE Trans. Plasma Sci. 137, 137-140.

Herro, H.M. and Port R.D., The Nalco guide to cooling water system failure analysis, McGraw Hill, New York, 1993.

Herron, J.T. and Green, D.S. (2001) Chemical Kinetics Database and Predictive Schemes for Nonthermal Humid Air Plasma Chemistry. Part II. Neutral Species Reactions Plasma Chemistry and Plasma Processing 21, 459-481.

Higashitani, K. and Oshitan, J. (1997) Measurements of magnetic effects on electrolyte by atomic force microscopy. Trans. I ChemE B 75, 115-119.

Hironori, A. (2008) Plasma generation inside externally supplied Ar bubbles in water. Plasma Sources Science and Technology 17(2), 025006.

Hurst, C.J. (2005) Manual of Environmental Microbiology, ASM Press, Washington, DC.

Incropera, F.P., Dewitt, D.P., Bergman, T.L. and Lavine, A.S. (2007) Fundamentals of heat and mass transfer, Wiley, New York.

Inoue, Y. and Kobayashi, T. (1993) Nonlinear Oscillation of a gas-filled spherical cavity in an incompressible fluid. Fluid Dyn. Res. 11, 85-97.

Ishijima, T. (2010) Efficient production of microwave bubble plasma in water for plasma processing in liquid. Plasma Sources Science and Technology 19(1), 015010. 
Itikawa, Y. (1974) Electron-Impact Vibrational Excitation of H2O. J. Phys. Soc. Jpn. 36, 11271132.

Itikawa, Y. and Mason, N. (2005) Cross Sections for Electron Collisions with Water Molecules J. Phys. Chem. Ref. Data 34, 1-22.

Jensen, M.J., Bilodeau, R.C., Heber, O., Pedersen, H.B., Safvan, C.P., Urbain, X., Zajfman, D. and Andersen, L.H. (1999) Dissociative recombination and excitation of $\mathrm{H} 2 \mathrm{O}+$ and $\mathrm{HDO}+$. Phys. Rev. A 60, 2970-2976.

Joanna, P. and Satoshi, I. (2007) Removal of Color Caused by Various Chemical Compounds Using Electrical Discharges in a Foaming Column. Plasma Processes and Polymers 4, 753759.

Jones, H.M. and Kunhardt, E.E. (1994) Influence of pressure and conductivity on the pulsed breakdown of water. IEEE Trans. Dielectr. Electr. Insul. 1, 1016-1025.

Jones, H.M. and Kunhardt, E.E. (1995) Development of Pulsed Dielectric Breakdown in Liquids. J. Phys. D: Appl. Phys. 28, 178-188.

Joseph, B.K. and Miksis, M. (1980) Bubble oscillations of large amplitude. J. Acoust. Soc. Am. 68, 628-633.

Joshi, A., Locke, B.R., Arce, P. and Finney, W.C. (1995) Formation of hydroxyl radicals, hydrogen peroxide and aqueous electrons by pulsed streamer corona discharge in aqueous solution. J. Haz. Mater. 41, 3-30.

Joshi, R., Qian, J., Zhao, G., Kolb, J.F. and Schoenbach, K.H. (2004) Are microbubbles necessary for the breakdown of liquid water subjected to a submicrosecond pulse? J. Appl. Phys. 96, 5129-5139.

Joshi, R.P., Kolb, J.F., Shu, X. and Schoenbach, K.H. (2009) Aspects of Plasma in Water: Streamer Physics and Applications. Plasma Processes and Polymers 6, 763-777.

Joshi, R.P., Qian, J. and Schoenbach, K.H. (2002b) Electrical network-based time dependent model of electrical breakdown in water. J. Appl. Phys. 92, 6245-6251.

Jun, Q., Joshi, R.P., Schoenbach, K.H., Woodworth, J.R. and Sarkisov, G.S. (2006) Model analysis of self- and laser-triggered electrical breakdown of liquid water for pulsed-power applications. IEEE Transactions on Plasma Science 34, 1680-1691.

Katsuki, S., Akiyama, H., Abou-Ghazala, A. and Schoenbach, K.H. (2002) Parallel streamer discharge between wire and plane electrodes in water. IEEE Trans. Dielect. Elect. Insul. 9, 498-506.

Katz, J.L., Fisk, J.A. and Chakarov, V.M. (1994) Condensation of a supersaturated vapor IX. Nucleation on ions. The Journal of Chemical Physics 101(3), 2309-2318.

Kenyon, K.E. (1983) On the depth of wave influence. J. Phys. Oceanogr. 13, 1968-1970.

Kenyon, K.E. (1998) Capillary waves understood by an elementary method. J. Oceanography 54, 343-346.

Khare, S.P. and Meath, W.J. (1987) Cross sections for the direct and dissociative ionisation of $\mathrm{NH} 3$, H 2 O and $\mathrm{H} 2 \mathrm{~S}$ by electron impact. Journal of Physics B: Atomic and Molecular Physics 20(9), 2101. 
Kim, W.T. (2001) A Study of Physical Water Treatment Methods for the Mitigation of Mineral Fouling, Drexel University, Philadelphia, PA.

Kline, S.J. and McClintock, F.A. (1953) Describing Uncertainties in Single-sample Experiments. Mech. Eng. 75, 3-8.

Kolb, J., Joshi, R., Xiao, S. and Schoenbach, K. (2008) Streamers in water and other dielectric liquids. J. Phys. D: Appl. Phys. 41, 234007.

Kolikov, V.A., Kurochkin, V.E., Panina, L.K. and Rutberg, F.G. (2005) Pulsed Electric Discharges and Prolonged Microbial Resistance of Water. Doklady Biological Sciences 403, 279-281.

Kolikov, V.A., Kurochkin, V.E., Panina, L.K., Rutberg, A.F., Rutberg, F.G., Snetov, V.N. and Stogov, A.Y. (2007) Prolonged Microbial Resistance of Water Treated by a Pulsed Electrical Discharge. Technical Physics 52(263-270).

Kong, M.G., Kroesen, G., Morfill, G., Nosenko, T., Shimizu, T., Dijk, J.v. and Zimmermann, J.L. (2009) Plasma medicine: an introductory review. New Journal of Physics 11(11), 115012.

Kontoyannis, C.G. and Vagena, N.V. (1999) Calcium carbonate phase analysis using XRD and FT-Raman spectroscopy. Analyst 125, 251-255.

Korobeynikov, S.M. and Melekhov, A.V. (2002) Microbubbles and breakdown initiation in water, Graz, Austria.

Kostyuk, P.V. (2008) Effect of $\mathrm{Ni}$ and $\mathrm{TiO} 2$ on hydrogen generation from aqueous solution with non-thermal plasma. Journal of Physics D: Applied Physics 41(9), 095202.

Krcma, F., Z., S. and J., P. (2010) Diaphragm Discharge in Liquids: Fundamentals and Applications. J. Phys.: Conference Series 207, 012010-012016.

Kupershtokh, A.L. and Medvedev, D.A. (2006) Anisotropic instability of dielectric liquids and decay to vapor-liquid system in strong electric fields. Tech. Phys. Lett. 32, 634-637.

Kushner, M.J. (1999) Strategies for Rapidly Developing Plasma Chemistry Model. Bull. Am. Phys. Soc. 44, 63.

Labas, M.D., Brandi, R.J., Martín, C.A. and Cassano, A.E. (2006) Kinetics of bacteria inactivation employing UV radiation under clear water conditions. Chemical Engineering Journal 121, 135-145.

Lama, W. and Gallo, C. (1977) Systematic study of the electrical characteristics of the tricel current pulses from negative needle-to-plane coronas. J. Appl. Phys. 45, 103-113.

Lange, H. and Huczko, A. (2004) Carbon Arc Discharge: Plasma Emission Spectroscopy and Carbon Nanostructure Formation. Trans. Mater. Res. Soc. Jpn. 29, 3359-3364.

Laroussi, M. (2005) Low temperature plasma-based sterilization: overview and state-of-the-art. Plasma Process Polym. 2, 391-400.

Laroussi, M. (2008) The Biomedical Applications of Plasma: A Brief History of the Development of a New Field of Research. Plasma Science, IEEE Transactions on 36(4), 1612-1614. 
Laroussi, M., Dobbs, F.C., Wei, Z., Doblin, M.A., Ball, L.G., Moreira, K.R., Dyer, F.F. and Richardson, J.P. (2002) Decontamination of water by excimer UV radiation. IEEE Trans. Plasma Sci. 30, 1501-1503.

Lee, G.J., Tijing, L.D., Pak, B.C., Baek, B.J. and Cho, Y.I. (2006) Use of catalytic materials for the mitigation of mineral fouling. Int. Commun. Heat Mass Transfer 33(1), 14-23.

Lee, H.Y., Uhm, H.S., Choi, H.N., Jung, Y.J., Kang, B.K. and Yoo, H.C. (2003) Underwater discharge and cell destruction by shockwaves. Journal of the Korean Physical Society 42, S880-S884.

Levine, I.N. (1978) Physical Chemistry, McGraw-Hill, New York.

Lewis, T. (1994) Basic electrical processes in dielectric liquids. IEEE Trans. Dielec. Dielectr. Electr. Insul. 1, 630-643.

Lewis, T. (1998) A New Model for the Primary Process of Electrical Breakdown in Liquids. IEEE Trans. Dielectr. Electr. Insul. 5, 306-315.

Lewis, T. (2003) Breakdown Initiating Mechanisms at Electrode Interfaces in Liquids. IEEE Trans. Dielect. Elect. Insul. 10, 948-955.

Lezzi, A. and Prosperetti, A. (1987) Bubble dynamics in a compressible liquid. II. Second-order theory. J. Fluid Mech. 185, 289-321.

Li, J., Sato, M. and Ohshima, T. (2007a) Degradation of phenol in water using a gas-liquid phase pulsed discharge plasma reactor. Thin Solid Films 515, 4283-4288.

Lide, D.R. (ed) (2005) CRC Handbook of Chemistry and Physics, CRC Press, Boca Raton.

Lin, L., Johnston, C.T. and Blatchley III, E.R. (1999) Inorganic fouling at quartz: water interfaces in ultraviolet photoreactors-I. chemical characterization. Water Res. 33, 33213329.

Lisitsyn, I.V., Nomiyama, H., Katsuki, S. and Akiyama, H. (1999a) Streamer discharge reactor for water treatment by pulsed power. Rev. Sci. Instrum. 70, 3457-3462.

Lisitsyn, I.V., Nomiyama, H., Katsuki, S. and Akiyama, H. (1999b) Thermal Processes in a Streamer Discharge in Water. IEEE Trans. Dielec. Dielectr. Electr. Insul. 6, 351-356.

Liu, Y.J. and Jiang, X.Z. (2005) Phenol Degradation by a Nonpulsed Diaphragm Glow Discharge in an Aqueous Solution. Environ. Sci. Technol. 39, 8512-8517.

Locke, B. R. , M. Sato, and P. Sunka etc., Electrohydraulic Discharge and Nonthermal Plasma for Water Treatment, Ind. Eng. Chem. Res. 452006 pp882-905

Locke, B.R., Burlica, R. and Kirkpatrick, M.J. (2006a) Formation of reactive species in gliding arc discharges with liquid water. Journal of Electrostatics 64(Copyright 2006, IEE), 35-43.

Loeb, L.B. (1960) Basic processes of gaseous electronics, University of California Press, Berkeley, CA.

Lu, X., Pan, Y. and Liu, K. (2002) Spark model of pulsed discharge in water. J. Appl. Phys. 91, 24-31.

Lukes, P., Clupek, M., Babicky, V. and Sunka, P. (2008) Pulsed electrical discharge in water generated using porous-ceramic-coated electrodes. IEEE Trans. Plasma Sci. 36, 1146-1147. 
Lukes, P., Clupek, M., Babicky, V. and Sunka, P. (2009) The Role of Surface Chemistry at Ceramic/Electrolyte Interfaces in the Generation of Pulsed Corona Discharges in Water Using Porous Ceramic-coated Rod Electrodes. Plasma Processes and Polymers 6, 719-728. Madigan, M.T. and Martinko, J.M. (2006) Biology of microorganisms, Prentice Hall, Upper Saddle River, NJ.

Magureanu, M., Piroi, D., Mandache, N.B., David, V., Medvedovici, A. and Parvulescu, V.I. (2010) Degradation of pharmaceutical compound pentoxifylline in water by non-thermal plasma treatment. Water Research 44(11), 3445-3453.

Malik, M.A., Minamitani, Y., Xiao, S., Kolb, J.F. and Schoenbach, K.H. (2005) Streamers in water filled wire-cylinder and packed bed reactors. IEEE Trans. Plasma Sci. 33, 490-491.

Matsushima, Y., Yamazaki, T., Maeda, K., Noma, T. and Suzuki, T. (2006) Plasma oxidation of a titanium electrode in dc-plasma above the water surface. Journal of the American Ceramic Society 89, 799-804.

Mededovic, S., Finney, W.C. and Locke, B.R. (2008) Electrical discharges in mixtures of light and heavy water. Plasma Processes and Polymers 5, 76-83.

Meek, J.M. and Craggs, J.D. (1978) Electrical breakdown of gases, Wiley, New York, NJ.

Miichi, T., Ihara, S., Satoh, S. and Yamabe, C. (2000) Spectroscopic measurements of discharges inside bubbles in water. Vacuum 59(1), 236-243.

Millar, T.J., Farquhar, P.R.A. and Willacy, K. (1997) The UMIST Database for Astrochemistry 1995. Astron. Astrophys. Suppl. Ser. 121, 139-185.

Mohd Abbas, N., Darius G. Solomon, Md. Fuad Bahari, A review on current research trends in electrical discharge machining, International Journal of Machine Tools \& Manufacture 47 2007 pp1214-1228

Moisan, M., Barbeau, J., Crevier, M.-C., Pelletier, J., Philip, N. and Saoudi, B. (2002) Plasma Sterilization. Methods and mechanisms. Pure Appl. Chem. 74(3), 349-358.

Moisan, M., Barbeau, J., Moreau, S., Pelletier, J., Tabrizian, M. and Yahia, L.H. (2001) Low temperature sterilization using gas plasmas: a review of the experiments and an analysis of the inactivation mechanisms. International Journal of Pharmaceutics 226, 1-21.

Morch, K.A. (2007) Reflections on cavitation nuclei in water. Phys. Fluids 19, 072104-072110.

Muhammad Arif, M. (2009) Water Purification by Plasmas: Which Reactors are Most Energy Efficient? Plasma Chemistry and Plasma Processing.

Muller-Steinhagen, H. (1999) Cooling water fouling in heat exchangers. Advanced Heat Transfer 33, 415-496.

Muller-Steinhagen, H. (2000) Handbook of Heat Exchanger Fouling - Mitigation and Cleaning Technologies, Publico Publications, Germany.

Nagasaka, Y. and Nagashima, A. (1981) Simultaneous measurement of the thermal conductivity and the thermal diffusivity of liquids by the transient hot-wire method. Rev. Sci. Instrum. 52, 229-232.

Nantel-Valiquette, M., Kabouzi, Y., Castaños-Martinez, E., Makasheva, K., Moisan, M. and Rostaing, J.C. (2006) Reduction of perfluorinated compound emissions using atmospheric 
pressure microwave plasmas: Mechanisms and energy efficiency. Pure Appl. Chem. 78, 1173-1185.

Nishioka, H., Saito, H. and Watanabe, T. (2009) Decomposition mechanism of organic compounds by DC water plasmas at atmospheric pressure. Thin Solid Films 518, 924-928.

Nomura, S. and Toyota, H. (2003) Sonoplasma generated by a combination of ultrasonic waves and microwave irradiation. Applied Physics Letters 83(22), 4503-4505.

Nomura, S., Toyota, H., Mukasa, S., Takahashi, Y., Maehara, T., Kawashima, A. and Yamashita, H. (2008) Discharge Characteristics of Microwave and High-Frequency InLiquid Plasma in Water. Applied Physics Express 1, 046002.

Nomura, S., Toyota, H., Mukasa, S., Yamashita, H., Maehara, T. and Kuramoto, M. (2006) Microwave plasma in hydrocarbon liquids. Applied Physics Letters 88(21), 211503-211503.

Olivero, J.J., Stagat, R.W. and Green, A. (1972) Electron Deposition in Water Vapor, with Atmospheric Applications. J. Geophys. Res. 77, 4797-4811.

Opalska A., Opalinska T. and P., O. (2002) Electroplasma-induced decomposition of chlorodifluoromethane under oxidizing conditions. Acta Agrophysica 80, 367-374.

Panchal, C.B. and Knudsen, J.G. (1998) Mitigation of water fouling: technology status and challenges. Advanced Heat Transfer 31, 431-474.

Parry, W., Bellows, J., Gallagher, J. and Harvey, A. (2008) ASME International Steam Tables for Industrial Use, The American Society of Mechanical Engineers, New York.

Parsons, S.A., Judd, S.J., Stephenson, T., Udol, S. and Wang, B.L. (1997) Magnetically augmented water treatment. Process Safety and Environmental Protection 75, 98-104.

Probstein, R.F. (1989) Physicochemical Hydrodynamics, Butterworths, Boston.

Prosperetti, A. and Lezzi, A. (1986) Bubble dynamics in a compressible liquid. I. First-order theory. J. Fluid Mech. 168, 457-478.

Qian, J., Joshi, R. and Schoenbach, K.H. (2006) Model Analysis of Self- and Laser-Triggered Electrical Breakdown of Liquid Water for Pulsed-Power Applications. IEEE Trans. Plasma Sci. 34, 1680-1691.

Qian, J., Joshi, R., Kolb, J.F. and Schoenbach, K.H. (2005) Microbubble-based model analysis of liquid breakdown initiation by a submicrosecond pulse. J. Appl. Phys. 97(113304113313).

Quan, Z., Chen, Y., Ma, C., Wang, C. and Li, B. (2009) Experimental study on anti-fouling performance in a heat exchanger with low voltage electrolysis treatment. Heat Transfer Engineering 30, 181-188.

Radler, S. and Ousko-Oberhoffer, U. (2005) Optimised heat exchanger management - achieving financial and environmental targets, Irsee, Germany.

Raether, H. (1964) Electron avalanches and breakdown in gases, Butterworth, London.

Raizer, Y.P. (1997) Gas Discharge Physics, Springer, Berlin.

Ram, N.M., Christman, R.F. and Cantor, K.P. (1990) Significance and treatment of volatile organic compounds in water supplies, CRC Press, Chelsea, MI. 
Rico, V., Hueso, J., Cotrino, J. and Gonzalez-Elipe, A. (2010) Evaluation of Different Dielectric Barrier Discharge Plasma Configurations As an Alternative Technology for Green C1 Chemistry in the Carbon Dioxide Reforming of Methane and the Direct Decomposition of Methanol. J. Phys. Chem. A 114, 4009-4016.

Robinson, J.W., Ham, M. and Balaster, A.N. (1973) Ultraviolet radiation from electrical discharges in water. J. Appl. Phys. 44, 72-75.

Rosario-Ortiz, F.L., Mezyk, S.P., Doud, D. and Snyder, S.A. (2008) Quantitative correlation of absolute hydroxyl radical rate constants with non-Isolated effluent organic matter bulk properties in water. Environ. Sci. Technol. 42, 5924-5930.

Sahni, M. and Locke, B.R. (2006) Quantification of reductive species produced by high voltage electrical discharges in water. Plasma Processes and Polymers 3, 342-354.

Sakiyama, Y., Tomai, T., Miyano, M. and Graves, D.B. (2009) Disinfection of E. coli by nonthermal microplasma electrolysis in normal saline solution. Applied Physics Letters 94(16).

Sato, K. and Yasuoka, K. (2008) Pulsed discharge development in oxygen, argon, and helium bubbles in water. IEEE Transactions on Plasma Science 36, 1144-1145.

Sato, K., Yasuoka, K. and Ishii, S. (2008a) Water treatment with pulsed plasmas generated inside bubbles. Transactions of the Institute of Electrical Engineers of Japan, Part A 128, 401-406.

Sato, K., Yasuoka, K. and Ishii, S. (2010) Water treatment with pulsed discharges generated inside bubbles. Electrical Engineering in Japan (English translation of Denki Gakkai Ronbunshi) 170, 1-7.

Sato, M. (2008) Environmental and biotechnological applications of high-voltage pulsed discharges in water. Plasma Sources, Science and Technology 17, 024021-024027.

Sato, M., Ohgiyama, T. and Clements, J.S. (1996) Formation of chemical species and their effects on microprganisms using a pulsed high-voltage discharge in water. IEEE Trans. Ind. Appl. 32, 106-112.

Sato, M., Tokutake, T., Ohshima, T. and Sugiarto, A.T. (2008b) Aqueous phenol decomposition by pulsed discharges on the water surface. IEEE Transactions on Industry Applications 44, 1397-1402.

Schoenbach, K., Joshi, R., Kolb, J., Chen, N., Stacey, M., Blackmore, P.F., Buescher, E.S. and Beebe, S.J. (2004) Ultrashort Electrical Pulses Open a New Gateway Into Biological Cells. Proc. IEEE 92(7), 1122-1137.

Schoenbach, K., Kolb, J., Shu, X., Katsuki, S., Minamitani, Y. and Joshi, R. (2008) Electrical breakdown of water in microgaps. Plasma Sources, Science and Technology 17, 024010 (024010 pp.).

Schoenbach, K.H., Hargrave, B., Joshi, R.P., Kolb, J.F., Nuccitelli, R., Osgood, C., Pakhomov, A., Stacey, M., Swanson, R.J., White, J.A., Shu, X., Jue, Z., Beebe, S.J., Blackmore, P.F. and Buescher, E.S. (2007) Bioelectric effects of intense nanosecond pulses. IEEE Transactions on Dielectrics and Electrical Insulation 14, 1088-1109. 
Schoenbach, K.H., Peterkin, F.E., Alden, R.W.J. and Beebe, S.J. (1997) The effect of pulsed electric fields on biological cells: experiments and applications. IEEE Trans. Plasma Sci. 25, 284-289.

Schutten, J., de Heer, F.J., Moustafa, H.R., Boerboom, A.J.H. and Kistemaker, J. (1966) Grossand Partial-Ionization Cross Sections for Electrons on Water Vapor in the Energy Range 0.1--20 keV. The Journal of Chemical Physics 44(10), 3924-3928.

Siegal, F. and Reams, M.W. (1966) Temperature effect on precipitation of calcium carbonate from calcium bicarbonate solutions and its application to cavern environments. Sedimentology 7, 241-247.

Smith, C., Coetzee, P.P. and Meyer, J.P. (2003) The effectiveness of a magnetic physical water treatment device on scaling in domestic hot-water storage tanks. Water SA 29, 231-236.

Smith, W.F. (1986) Principles of Materials Science and Engineering, McGraw-Hill Book Co., Singapore.

Snizhko, L.O., Yerokhin, A.L., Gurevina, N.L., Patalakha, V.A. and Matthews, A. (2007) Excessive oxygen evolution during plasma electrolytic oxidation of aluminium. Thin Solid Films 516, 460-464.

Snoeyink, V.L. and Jenkins, D. (1982) Water Chemistry, Wiley, New York.

Somerscales, E.F.C. (1990) Fouling of heat exchangers: an historical review. Heat Transfer Engineering 11(1), 19-36.

Sridharan, K., Harrington, S.P., Johnson, A.K., Licht, J.R., Anderson, M.H. and Allen, T.R. (2007) Oxidation of plasma surface modified zirconium alloy in pressurized high temperature water. Materials \&amp; Design 28, 1177-1185.

Staack, D., Fridman, A., Gutsol, A., Gogotsi, Y. and Friedman, G. (2008) Nanoscale Corona Discharge in Liquids, Enabling Nanosecond Optical Emission Spectroscopy. Angew. Chem. Int. Ed. 47, 8020-8024.

Stalder, K.R., Mcmillen, D.F. and Woloszko, J. (2005) Electrosurgical plasmas. J. Phys. D: Appl. Phys. 38, 1728-1738.

Starikovskiy, A., Yang, Y., Cho, Y.I. and Fridman, A. (2011) Non-equilibrium plasma in liquid water: Dynamics of generation and quenching. Plasma Sources Sci. Technol. 20, 024003.

Straub, H.C., Lindsay, B.G., Smith, K.A. and Stebbings, R.F. (1998) Absolute partial cross sections for electron-impact ionization of $\mathrm{H}[\mathrm{sub} 2] \mathrm{O}$ and $\mathrm{D}$ [sub 2]O from threshold to 1000 eV. The Journal of Chemical Physics 108(1), 109-116.

Sugiarto, A.T. and Sato, M. (2001) Pulsed plasma processing of organic compounds in aqueous solution. Thin Solid Films 386, 295-299.

Sugiarto, A.T., Ohshima, T. and Sato, M. (2002) Advanced oxidation processes using pulsed streamer corona discharge in water. Thin Solid Films 407, 174-178.

Sun, B., Kunitomo, S. and Igarashi, C. (2006) Characteristics of ultraviolet light and radicals formed by pulsed discharge in water. Journal of Physics D-Applied Physics 39(17), 38143820 . 
Sun, B., Sato, M. and Clements, J.S. (1997) Optical study of active species produced by a pulsed streamer corona discharge in water. J. Electrostat. 39, 189-132.

Sun, B., Sato, M., Harano, A. and Clements, J.S. (1998) Non-uniform pulse discharge-induced radical production in distilled water. J. Electrostat 43, 115-126.

Sunka, P. (2001) Pulse electrical discharges in water and their applications. Phys Plasmas 8, 2587-2594.

Sunka, P., Babicky, M., Clupek, M., Fuciman, M., Lukes, P., Simek, M., Benes, J., Locke, B. and Majcherova, Z. (2004) Potential applications of pulse electrical discharges in water. Acta Physica Slovaca 54, 135-145.

Sunka, P., Babicky, V., Clupek, M., Lukes, P. and Balcarova, J. (2003) Modified pinhole discharge for water treatment. Pulsed Power Conf. Digest of Technical Papers 1, 229-231.

Sunka, P., Babicky, V., Clupek, M., Lukes, P., Simek, M., Schmidt, J. and Cernak, M. (1999) Generation of chemically active species by electrical discharges in water. Plasma Sources Sci. Technol. 8, 258-265.

Suzuki, T., Saburi, T., Tokunami, R., Murata, H. and Fujii, Y. (2006) Dominant species for oxidation of stainless steel surface in water vapor plasma. Thin Solid Films 506-507, 342345.

Takeda, T., Jen-Shih, C., Ishizaki, T., Saito, N. and Takai, O. (2008) Morphology of HighFrequency Electrohydraulic Discharge for Liquid-Solution Plasmas. Plasma Science, IEEE Transactions on 36(4), 1158-1159.

Test, T. (2000) plasma in water. IEEE Trans. Dielectr. Electr. Insul. 40, 125-136.

The US Department of Energy (1998) Non-chemical technologies for scale and hardness control. DOE/EE-0162.

Tijing, L.D., Kim, H., Kim, C., Lee, D. and Cho, Y.I. (2009) Use of an oscillating electric field to mitigate mineral fouling in a heat exchanger. Experimental Heat Transfer 22, 257-270.

Trunin (1994) Shock compressibility of condensed materials in strong shock waves generated by underground nuclear explosions Physics-Uspekhi 37, 1123.

United States Environmental Protection Agency Office of Water (2009) National Water Quality Inventory: Report to Congress. 2004 Reporting Cycle.

Ushakov, G.V. (2008) Antiscaling treatment of water by an electric field in heat-supply networks. Thermal Engineering 55, 570-573.

Vankov, A. and Palanker, D. (2007) Nanosecond plasma-mediated electrosurgery with elongated electrodes. Journal of Applied Physics 101(12).

Volenik, K., Nop, P., Kopoeiva, P., Kolman, B. and Dubsky, J. (2006) Isothermal oxidation of metallic coatings deposited by a water-stabilized plasma gun. Metallic Materials 44, 40-48.

Wait, I.W. (2005) Fouling of quartz surfaces in potable water ultraviolet disinfection reactors, Purdue University.

Watson, P. and Chadbank, W. (1991) The Role of Electrostatic and Hydrodynamic Forces in the Negative-point Breakdown of Liquid Dielectrics. IEEE Trans. Electr. Insul. 26, 543-559. 
Weast, R. and David, R.L. (1986) Handbook of Chemistry and Physics, CRC Press, Boca Raton, FL.

White, F.M. (2006) Viscous Fluid Flow, McGraw-Hill Companies, Singapore.

Wilson, M.P., Balmer, L. and Given, M.J. (2006) Application of electric spark generated high power ultrasound to recover ferrous and non-ferrous metals from slag waste. Minerals Engineering 19, 491-499.

Wolfe, R.L. (1990) Ultraviolet disinfection of potable water. Environ. Sci. Technol. 24, 768-773.

Woodworth, J.R., Lehr, J.M. and Elizondo-Decanini, J. (2004) Optical and Pressure Diagnostics of 4-MV Water Switches in the Z-20 Test Facility. IEEE Trans. Plasma Sci. 32, 1778-1789.

World Health Organization (2010) Progress on sanitation and drinking-water.

World Water Assessment Programme (2009) World Water Assessment Programme. The 3rd United Nations World Water Development Report: Water in a Changing World, UNESCO, Paris.

Xiaokai, X. (2008) Research on the electromagnetic anti-fouling technology for heat transfer enhancement. Appl. Therm. Eng. 28, 889-894.

Xiaokai, X., Chongfang, M. and Yongchang, C. (2005) Investigation on the electromagnetic anti-fouling technology for scale prevention. Chem. Eng. Technol. 28, 1540-1545.

Xie, H., Gu, H., Fujii, M. and Zhang, X. (2006) Short hot wire technique for measuring thermal conductivity and thermal diffusivity of various materials. Meas. Sci. Technol. 17, 208-214.

Yan, Z.C., Li, C. and Lin, W.H. (2009) Hydrogen generation by glow discharge plasma electrolysis of methanol solutions. Int. J. Hydrogen Energy 34, 48-55.

Yang, Y., Gutsol, A., Fridman, A. and Cho, Y.I. (2009b) Removal of CaCO3 scales on a filter membrane using plasma discharge in water. Int. J. Heat Mass Transfer 52, 4901-4906.

Yang, Y., Kim, H., Fridman, A. and Cho, Y.I. (2010b) Effect of a plasma-assisted self-cleaning filter on the performance of PWT coil for the mitigation of mineral fouling in a heat exchanger. Int. J. Heat Mass Transfer 53, 412-422.

Yang, Y., Kim, H., Starikovskiy, A., Fridman, A. and Cho, Y.I. (2010a) Application of pulsed spark discharge for calcium carbonate precipitation in hard water. Water Res. 44, 36593668 .

Yang, Y., Kim, H., Starikovskiy, A., Fridman, A. and Cho, Y.I. (2011a) Pulsed multichannel discharge array in water with stacked circular disk electrodes. IEEE Trans. Plasma Sci., (In press, DOI:10.1109/TPS.2011.2129600).

Yang, Y., Kim, H., Starikovskiy, A., Fridman, A. and Cho, Y.I. (2011b) Precipitation of calcium ions from hard water using pulsed spark discharges and its mechanism. Plasma Chemistry and Plasma Processing 31, 51-66.

Yang, Y., Kim, H., Starikovskiy, A., Fridman, A. and Cho, Y.I. (2011c) Application of pulsed spark discharge for mineral fouling mitigation in a heat exchanger. J. Heat Transfer 133, 054502 .

Yang, Y., Starikovskiy, A., Fridman, A. and Cho, Y.I. (2011d) Analysis of streamer propagation for electric breakdown in liquid/bioliquid. Plasma Medicine 1, 65-83. 
Yong Cheol, H., Hyun Jae, P., Bong Ju, L., Won-Seok, K. and Han Sup, U. (2010) Plasma formation using a capillary discharge in water and its application to the sterilization of $\mathrm{E}$. coli. Physics of Plasmas 17, 053502-053506.

Yukhymenko, V.V., Chernyak, V.Y. and Olshevskii, S.V. (2008) Plasma conversion of ethanolwater mixture to synthesis gas. Ukr. J. Phys. 53, 409-413.

Zhang, R.B., Wang, L., Wu, Y., Guan, Z. and Jia, Z. (2006) Bacterial decontamination of water by bipolar pulsed discharge in a gas-liquid-solid three-phase discharge reactor. IEEE Transactions on Plasma Science 34, 1370-1374. 


\title{
Application of pulsed spark discharge for calcium carbonate precipitation in hard water
}

\author{
Yong Yang, Hyoungsup Kim, Andrey Starikouskiy, Alexander Fridman, Young I. Cho* \\ Department of Mechanical Engineering and Mechanics, Drexel University, Philadelphia, PA 19104, USA
}

A R T I C L E I N F O

Article history:

Received 8 February 2010

Received in revised form

12 April 2010

Accepted 22 April 2010

Available online 29 April 2010

Keywords:

Physical water treatment

Plasma

Spark discharge

Hard water

Calcium carbonate precipitation

\begin{abstract}
A B S T R A C T
The effect of underwater pulsed spark discharge on the precipitation of dissolved calcium ions was investigated in the present study. Water samples with different calcium hardness were prepared by continuous evaporation of tap water using a laboratory cooling tower. It was shown that the concentration of calcium ions dropped by $20-26 \%$ after 10 -min plasma treatment, comparing with no drop for untreated cases. A laser particle counting method demonstrated that the total number of solid particles suspended in water increased by over $100 \%$ after the plasma treatment. The morphology and the crystal form of the particles were identified by both scanning electron microscopy and X-ray diffraction. Calcite with rhombohedron morphology was observed for plasma treated cases, comparing with the round structure observed for no-treatment cases. It was hypothesized that the main mechanisms for the plasma-assisted calcium carbonate precipitation might include electrolysis, local heating in the vicinity of plasma channel and a high electric field at the tip of plasma streamers, inducing structural changes in the electric double layer of hydrated ions.
\end{abstract}

(c) 2010 Elsevier Ltd. All rights reserved.

\section{Introduction}

According to the U.S. Geological Survey's (USGS) water use survey data (Feeley et al., 2008), thermoelectric generation accounted for 39\% (136 billion gallons per day) of all freshwater withdrawals in U.S. in 2000 , second only to irrigation. Each kilowatt-hour (kWh) of thermoelectric generation requires the withdrawal of approximately 25 gallons of water, which is primarily consumed for cooling purposes.

Since heat removal from condenser tubes requires the evaporation of pure water, the concentration of mineral ions such as calcium and magnesium in the circulating cooling water increases with time. Even though the makeup water is relatively soft, the continuous circulation eventually increases the hardness of the water due to pure water evaporation. These mineral ions, when transported through piping in ordinary plumbing system, can cause various problems, including the loss of heat transfer efficiencies in condensers and pipe clogging due to scale formation (Somerscales, 1990; Panchal and Knudsen, 1998). Thus, in order to maintain a certain calcium hardness level in the cooling water, one must discharge a fraction of water through blowdown and replace it with makeup water. In a typical cooling tower application, the cycle of concentration (COC) in cooling water is often maintained at 3.5. That means if the calcium carbonate hardness of the makeup water is $100 \mathrm{mg} / \mathrm{L}$, the hardness in the circulating cooling water is maintained at approximately $350 \mathrm{mg} / \mathrm{L}$. If the COC can be increased through continuous precipitation and removal of calcium ions, one can significantly reduce the amounts of makeup and blowdown water, resulting in the conservation of freshwater.

Various chemical and non-chemical methods are used to prevent scaling and thus increase the COC. Among them the scale-inhibiting chemicals like chlorine and brominated

\footnotetext{
* Corresponding author. Tel.: +1 215895 2425; fax: +1 2158951478.

E-mail address: choyi@drexel.edu (Y.I. Cho).
}

0043-1354/\$ - see front matter (c) 2010 Elsevier Ltd. All rights reserved.

doi:10.1016/j.watres.2010.04.024 
compounds were the best choice for the control of mineral fouling. Aside from the high cost of chemicals, more stringent environmental laws increased the costs associated with their storage, handling and disposal. These chemicals also pose danger on human health and environment with accidental spills, or accumulated chemical residues over a long period of time (Panchal and Knudsen, 1998; Muller-Steinhagen, 2000). Thus, there is a need for new approach which is safe and economical from both environmental and cost points of view in cleaning and maintenance of heat exchangers. Physical water treatment (PWT) is a non-chemical method to mitigate mineral fouling with the use of electric or magnetic fields, catalytic surfaces, ultrasounds, or sudden pressure changes. Numerous studies have been reported for the effectiveness of ultrasound (Radler and Ousko-Oberhoffer, 2005), solenoid coils (Cho et al., 2003; Cho et al., 2004), magnetic fields (Fathi et al., 2006; Xiaokai, 2008), catalytic material (Coetzee et al., 1998; Lee et al., 2006), and electrolysis (Gabrielli et al., 2006).

In recent years, there is an increasing interest in the study of pulsed electric breakdown in water and other liquids as it finds more applications in both industry and academic researches. High-voltage electrical discharges directly in water have been shown to be able to induce various reactions including the degradation of organic compounds, the destruction of bacteria and viruses, the oxidation of inorganic ions, the synthesis of nanomaterials and polymers (Akiyama, 2000; Sunka, 2001; Locke et al., 2006). The reactions are usually thought to be initiated by various reactive species, UV radiation, shockwaves, high electric field or intense heat produced by pulsed electric discharge. The concentration of the reactive species and the intensity of the physical effects largely depend on the discharge type and solution properties.

Herein the present study reports for the first time the pulsed spark discharge-assisted precipitation of dissolved calcium ions in hard water system. By measuring the variations of calcium and bicarbonate ion concentrations, the solution $\mathrm{pH}$, and the size and number of solid particles suspended in water, the effect of the spark discharge treatment on the nucleation precipitation of calcium carbonate was studied. The morphology of the precipitates was examined by scanning electron microscopy, whereas their crystal structure was identified by X-ray diffraction.

\section{Experiment}

\subsection{Water preparation}

To simulate the actual situations in the field, a closed-loop laboratory cooling tower was utilized in the study where Philadelphia city tap water was recirculated to produce concentrated hard water. Fig. 1 shows the schematic diagram of the cooling tower. The recirculation system consisted of a blower to supply air to the cooling tower, a heater to heat the air, a pump to circulate the water, and the cooling tower itself which was filled with Styrofoam balls used as strainers. As pure water evaporated in the cooling tower, the mineral ions such as calcium and magnesium were left behind and collected in the reservoir. Thus, with the continuous recirculation of hard water in the cooling tower, the concentration of

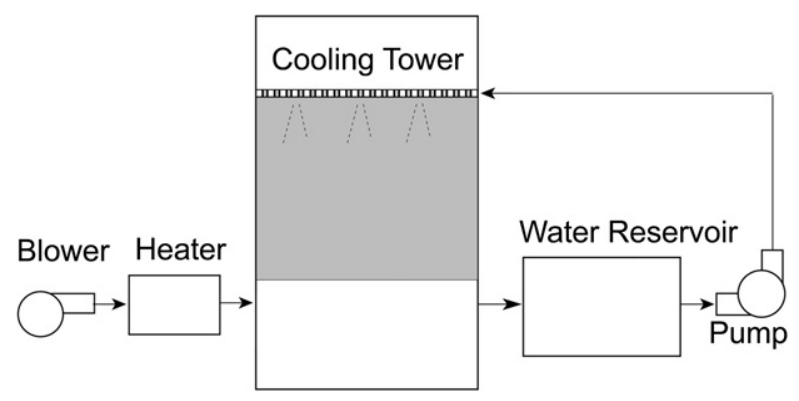

Fig. 1 - Schematic diagram of the laboratory cooling tower.

mineral ions was gradually increased until desired calcium carbonate hardness of $200-500 \mathrm{mg} / \mathrm{L}$ was reached. It usually took several days to obtain the desired concentration. The water $\mathrm{pH}$ resulting from this preparation was in the range of 8-10. A total of $100 \mathrm{~L}$ of concentrated hard water for each sample was prepared and kept in the reservoir and used for the whole duration of the experiment. The concentrations of cations and anions were analyzed, and Table 1 summarizes the chemical compositions of different water samples collected from the laboratory cooling tower.

\subsection{Pulsed spark discharge generation system}

A pulsed power system was constructed to produce spark discharge directly in water. The system consisted of three components: a high-voltage power supply with a capacitive energy storage, a spark-gap based switch, and a point-to-plain electrode system immersed in water. A schematic diagram of the pulsed power system is shown in Fig. 2. An 8.5-nF capacitor bank was charged by high-voltage pulses provided by a pulsed power supply. When triggered by an air-filled sparkgap switch, spark discharge was initiated between the electrodes from the overvoltage produced by the capacitor. The electrode system included a stainless steel 316-wire electrode as anode, and a stainless steel disk as grounded cathode. The radii of the anode and the disk cathode were 2 and $40 \mathrm{~mm}$, respectively. The radius of curvature at the tip of the anode was $0.2 \mathrm{~mm}$. The distance between the anode and the disk cathode was $10 \mathrm{~mm}$.

Power deposited into water was analyzed by measuring the current passing through the discharge gap and the voltage drop in the gap. For measurements of the current, a magnetic-

Table 1 - Chemical compositions of water sample collected in the simulated laboratory cooling tower.

\begin{tabular}{lccc} 
& Sample 1 & Sample 2 & Sample 3 \\
\hline $\mathrm{Ca}^{2+}$ & 96 & 128 & 167 \\
$\mathrm{Na}^{+}$ & 156 & 230 & 297 \\
$\mathrm{Mg}^{2+}$ & 8 & 13 & 17 \\
$\mathrm{Cl}^{-}$ & 98 & 140 & 186 \\
$\mathrm{SO}_{4}^{-}$ & 176 & 269 & 362 \\
$\mathrm{HCO}_{3}^{-}$ & 296 & 392 & 498 \\
$\mathrm{pH}$ & 8.67 & 9.10 & 9.62 \\
$\mathrm{CaCO}_{3}$ hardness & 279 & 378 & 492 \\
All values are in mg/L. & & & \\
\hline
\end{tabular}




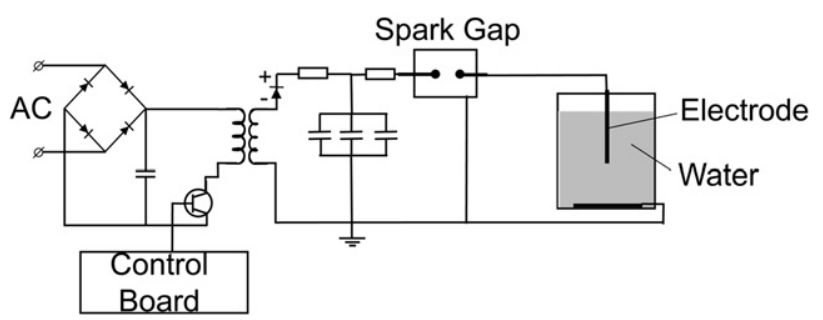

Fig. 2 - Schematic diagram of the pulsed power system used in the present study.

core Pearson current probe was utilized ( $1 \mathrm{~V} / \mathrm{Amp}+1 /-0 \%$ sensitivity, $10 \mathrm{~ns}$ usable rise time, and $35 \mathrm{MHz}$ bandwidth). Voltage was measured using a wide bandwidth 1:1000 voltage probe (PVM-4, North Star Research Corp.). Signals from the current and voltage probes were acquired and recorded by a digital phosphor oscilloscope (DPS) $(500 \mathrm{MHz}$ bandwidth, $5 \times 10^{9}$ samples/s, TDS5052B, Tektronix). Acquired data were then integrated using a customized MATLAB code.

Typical voltage and current waveforms are shown in Fig. 3. A fast rise time ( $\sim 9 \mathrm{~ns})$ was obtained with the closure of a spark-gap switch. The peak-to-peak voltage was about $24 \mathrm{kV}$. The pre-breakdown time, i.e. the time required before the spark formation was about $8.8 \mu \mathrm{s}$. During the pre-breakdown time, the energy was mostly consumed by ionic current due to the high conductivity of water samples. After that the abrupt increase in current was observed. This may possibly indicate that spark was formed as the streamer reached the other electrode and formed the highly conductive channel. The Full-Width at Half-Maximum (FWHM) of the major current pulse during spark discharge was $1.1 \mu \mathrm{s}$. The typical peak-to-peak breakdown current was $98 \mathrm{~A}$. It is worth to mention that the energy dissipated in pre-breakdown current can be comparable with, or even higher than the energy deposited in spark, especially at high-conductivity water conditions. The pulsed energy stored in the capacitor $E_{b}$ was about $1.7 \mathrm{~J}$, which was calculated by.

$E_{\mathrm{b}}=0.5 C \mathrm{~V}_{\mathrm{b}}^{2}$

where $C$ was $8.5 \mathrm{nF}$, and $V_{b}$ the capacitor voltage was $20 \mathrm{kV}$. By integrating the voltage and current, the energy deposited into the spark discharge was calculated as

$E_{s}=\int_{t_{1}}^{t_{2}} V(t) I(t) d t$

where $V(t)$ and $I(t)$ are the voltage and current measured by the oscilloscope, respectively, $t_{1}$ and $t_{2}$ are the starting and ending points of the spark, respectively. Similarly, the energy dissipated in the pre-breakdown stage was calculated as

$E_{p}=\int_{t_{0}}^{t_{1}} V(t) I(t) d t$

where $t_{0}$ is the time when the high voltage was applied. The values of $E_{\mathrm{s}}$ and $E_{\mathrm{p}}$ were approximately $0.69 \mathrm{~J} /$ pulse and $0.81 \mathrm{~J} /$ pulse, respectively, indicating that about $88 \%$ of the energy stored in the capacitor was deposited in water. The rest of the energy was possibly dissipated in the circuit during the discharge process.

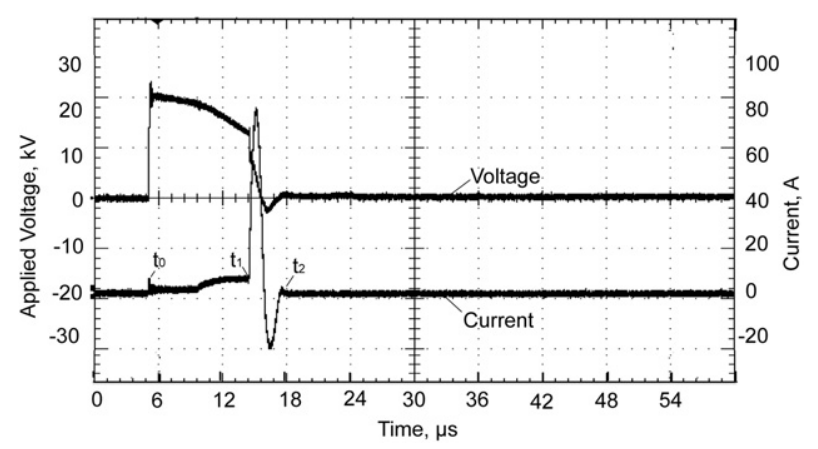

Fig. 3 - Typical voltage and current waveform of the spark discharge in water.

\subsection{Water treatment}

Water samples with different initial calcium hardness (i.e. Samples 1-3) were treated by spark discharge in a PTFE cylinder. In each run, the sample solution of $1 \mathrm{~L}$ was exposed to the discharge for $20 \mathrm{~min}$. The frequency of the discharge was set at $2 \mathrm{~Hz}$. Hence, the total energy deposited into the 1-L sample during a 20-min treatment can be calculated as about $3600 \mathrm{~J}$. The temperature difference before and after treatment was less than $1.5^{\circ} \mathrm{C}$, so it can be concluded that no significant bulk heating was caused by the spark discharge. A small sample solution of $10 \mathrm{ml}$ was extracted every $5 \mathrm{~min}$ for $\mathrm{pH}$ and ion concentration measurements. A filter with $0.5-\mu \mathrm{m}$ pore size was used to remove suspended $\mathrm{CaCO}_{3}$ from the sample. After filtration calcium ion concentration was measured by a standard ethylenediaminetetraacetic acid (EDTA) titration method, and the concentration of bicarbonate ion was determined by both total alkalinity test and phenolphthalein alkalinity test (Snoeyink and Jenkins, 1982). Untreated samples were collected from the stock solution at the same time intervals in order to evaluate the loss of calcium as a result of the natural precipitation process. All the experiments were replicated 3 times and average values were calculated.

Before and at the end of each experiment, a laser particle counter (Spectrex Model PC-2200) was used to count the size distribution of particles in suspension. After that, the solids were filtered with a pressurized filter $(0.5-\mu \mathrm{m}$ pore size) and dried in vacuum. Scanning electron microscopy (SEM) images and X-ray diffraction (XRD) analyses were carried out at Drexel SEM and XRD laboratories. SEM (FEI XL30) images were obtained to examine the topography of the deposits. Prior to each SEM examination, the deposits samples were coated by platinum through low-vacuum sputter coating in order to prevent the accumulation of static electric charge during the irradiation of electrons. XRD (Siemens D500) analyses were conducted to characterize the crystallographic structure of the scale deposits.

\section{Results}

Fig. 4 presents the variation of $\mathrm{pH}$ and calcium ion concentration as a function of time and input plasma energy, and comparisons with no-treatment cases. For the case of Sample 

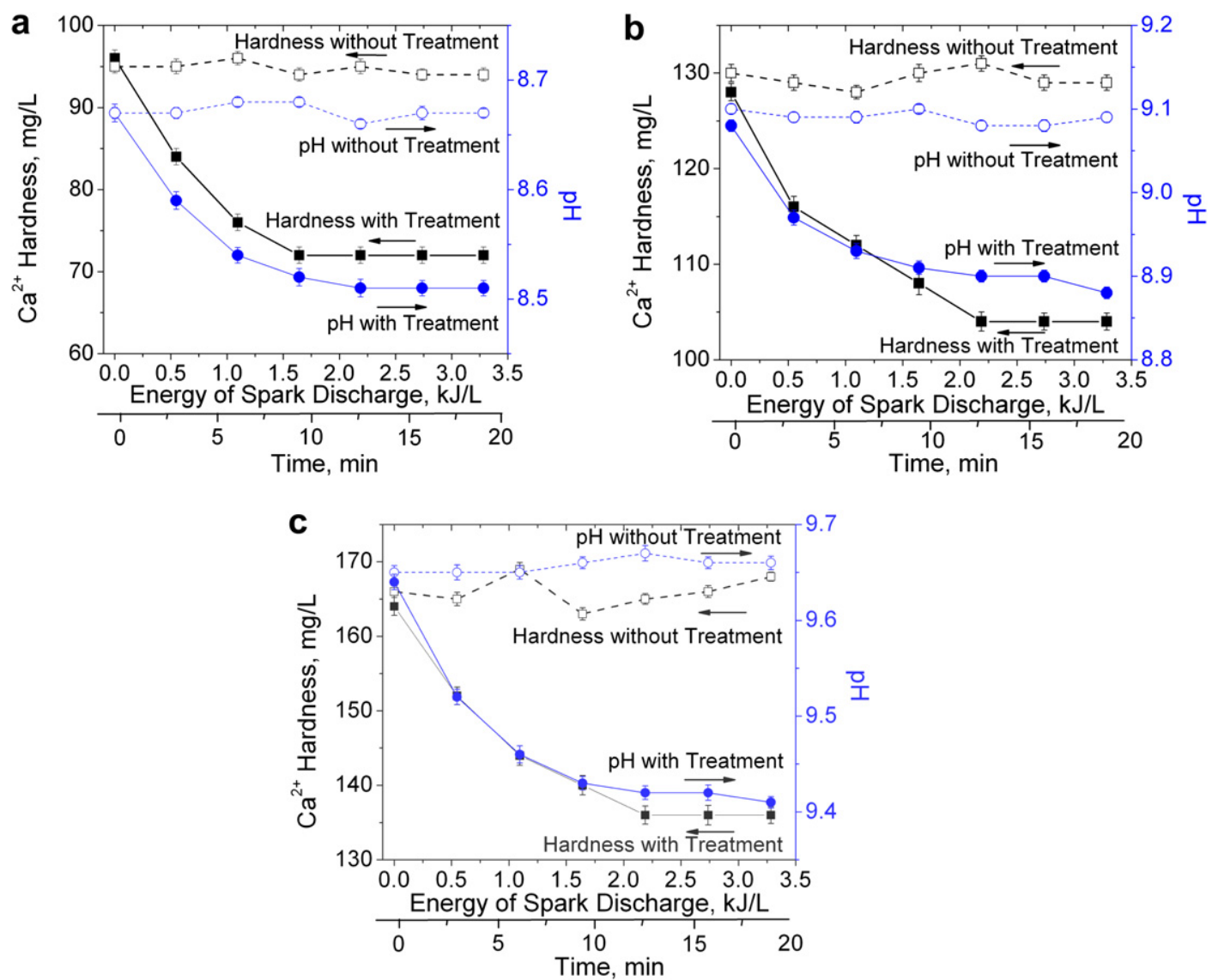

Fig. 4 - Comparison of $\mathrm{pH}$ and $\mathrm{Ca}^{2+}$ Hardness variation with and without plasma treatment: (a) Sample 1; (b) Sample 2; (c) Sample 3.

1, the calcium ion concentration dropped from an initial value of $96 \mathrm{mg} / \mathrm{L}$ to $71 \mathrm{mg} / \mathrm{L}$ after $10 \mathrm{~min}$ of plasma treatment. After that the hardness reached an asymptotic value and did not decrease with further input of energy. Accompanying the drop of the calcium ion concentration, the $\mathrm{pH}$ of the treated water sample decreased from 8.67 to 8.51 , possibly because of the liberation of $\mathrm{H}^{+}$ions according to:

$\mathrm{Ca}^{2+}+\mathrm{HCO}_{3}^{-} \rightarrow \mathrm{CaCO}_{3}+\mathrm{H}^{+}$

Additionally, the ionization of water molecules by highenergy electrons from plasma discharge may also contribute to the decrease of the $\mathrm{pH}$ through the following reaction:

$\mathrm{H}_{2} \mathrm{O}+\mathrm{e} \rightarrow \mathrm{H}_{2} \mathrm{O}^{+}+2 \mathrm{e}$

$\mathrm{H}_{2} \mathrm{O}^{+}+\mathrm{H}_{2} \mathrm{O} \rightarrow \mathrm{H}_{3} \mathrm{O}^{+}+\mathrm{OH}^{*}$

Samples 2 and 3, with initial calcium ion concentrations of 128 and $164 \mathrm{mg} / \mathrm{L}$, respectively, showed similar trends as Sample 1. For both samples, the hardness was reduced by about $25 \%$ after 10 -min treatment, with an energy input of approximately $1800 \mathrm{~J} / \mathrm{L}$. In contrast to the plasma treated cases, no significant change in the calcium ion concentration was observed for the no-treatment cases.

Fig. 5 presents the variations of bicarbonate ion concentration with time determined by total alkalinity test and phenolphthalein alkalinity test. Generally, the concentration of bicarbonate ion decreased during a 10-min stabilization period before reaching an asymptotic value. Similar to the changes in the concentration of calcium ions, the bicarbonate
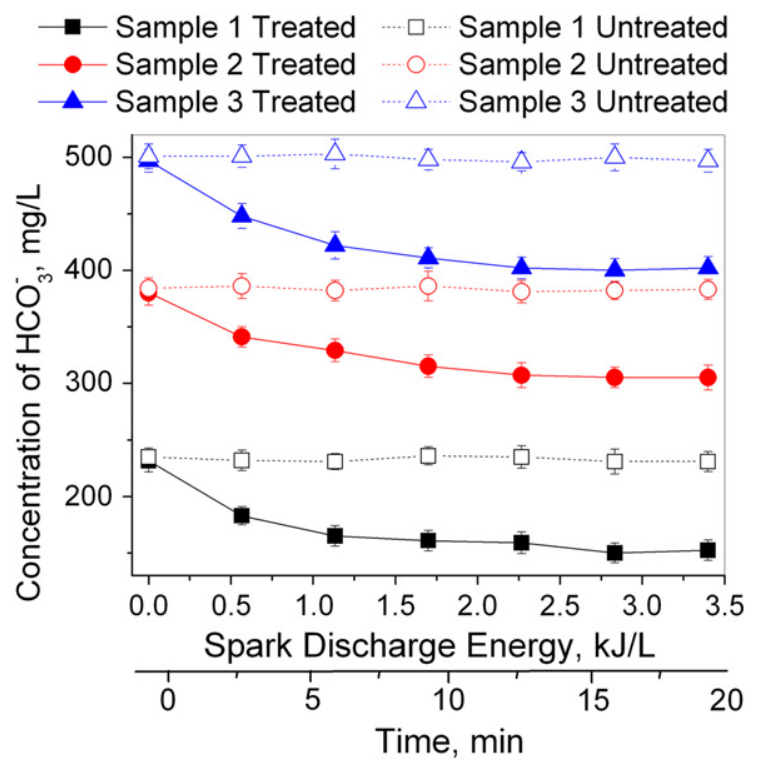

Fig. 5 - Comparison of $\mathrm{HCO}_{3}^{-}$variation for cases with and without plasma treatment. 

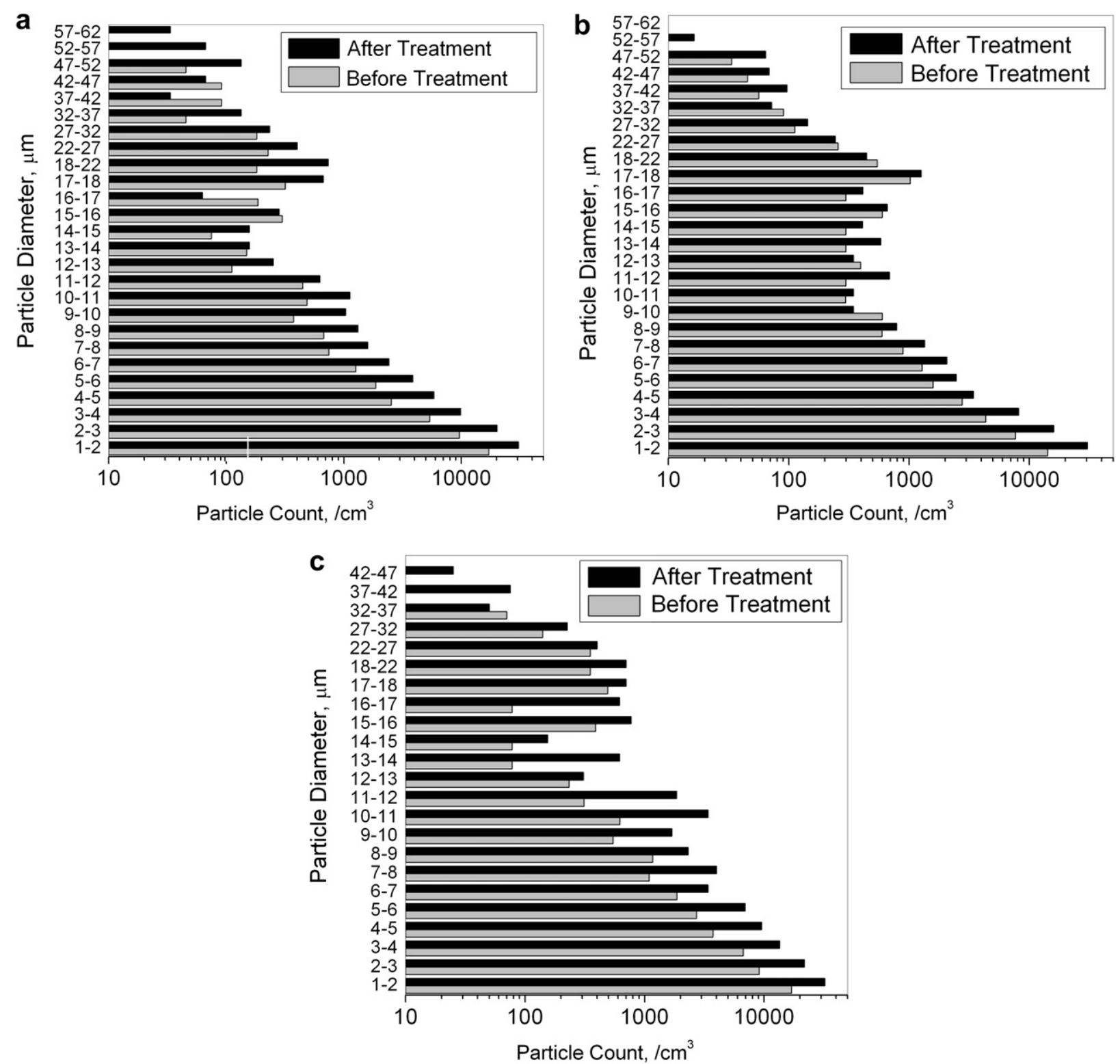

Fig. 6 - Comparison of particle size distribution before and after plasma treatment for water with initial hardness of: (a) Sample 1; (b) Sample 2; (c) Sample 3.

ion concentration changed very little for energy input greater than $2 \mathrm{~kJ} / \mathrm{L}$. It was probably because the rate of precipitation reactions was limited by the diffusion rate of calcium and bicarbonate ions towards the reaction zone due to the high localization of the plasma discharge. In addition, the acidification of the water sample at the vicinity of the anode by plasma-induced $\mathrm{H}^{+}$generation (Eqs. (5) and (6)) could become detrimental to the precipitation process.

Fig. 6 shows the number of particles per unit volume of $1 \mathrm{ml}$ as a function of particle size before and after plasma treatment. The results depict in general that before the treatment, the number of particles less than $10 \mu \mathrm{m}$ was significantly greater than that of larger particles (greater than $10 \mu \mathrm{m})$. For example, for Sample 1 (Fig. 6a), the number of particles with size between 1 and $2 \mu \mathrm{m}$ was 17,048 before treatment as compared to 2445 for particles greater than $10 \mu \mathrm{m}$. After treatment, a significant increase in the number of particles was observed for all cases as compared to the no-treatment cases. For Sample 1, the number of particles with size between 1 and $10 \mu \mathrm{m}$ increased from 39,904 at the initial state to 77,680 at the end of the treatment, while the number of particles with size over $10 \mu \mathrm{m}$ increased from 2445 to 3529. For Samples 2 and 3 (Fig. 6(b) and (c)), particles smaller than $10 \mu \mathrm{m}$ also made up the majority of the suspended solids in water. The number of small particles (i.e. below $10 \mu \mathrm{m}$ ) after the treatment was significantly increased comparing with that obtained for no-treatment cases.

Assuming that all the particles are in the spherical shape for the purpose of mathematical estimation, the total mass of suspended solid contents $m$ was calculated using the following equation: 


\begin{tabular}{|c|c|c|c|}
\hline & $\underset{1}{\text { Sample }}$ & $\begin{array}{l}\text { Sample } \\
2\end{array}$ & $\underset{3}{\text { Sample }}$ \\
\hline $\begin{array}{l}\text { Total number of particles before } \\
\text { treat, } \mathrm{cm}^{-3}\end{array}$ & 42,349 & 38,587 & 47,034 \\
\hline $\begin{array}{l}\text { Mass of suspended particles before } \\
\text { treat }\end{array}$ & 64 & 72 & 53 \\
\hline $\begin{array}{l}\text { Total number of particles after treat, } \\
\mathrm{cm}^{-3}\end{array}$ & 81,710 & 71,340 & 105,846 \\
\hline $\begin{array}{l}\text { Mass of suspended particles after } \\
\text { treat }\end{array}$ & 104 & 120 & 108 \\
\hline Increase of solid mass in water & 40 & 48 & 56 \\
\hline $\begin{array}{l}\text { Decrease of ionic } \mathrm{CaCO}_{3} \text { hardness in } \\
\text { water }\end{array}$ & 62 & 67 & 75 \\
\hline
\end{tabular}

$m=\sum_{N_{d}(d=1 \mu \mathrm{m})}^{\mathrm{N}_{d}(d=62 \mu \mathrm{m})} \rho \cdot \frac{\pi d^{3}}{6} \cdot \mathrm{N}_{d}$

where $\rho$ was the density of the $\mathrm{CaCO}_{3}$ particle, $d$ was the diameter of the particle, $N_{d}$ was the number of particles. $\rho$ was taken as $2.7 \mathrm{~g} / \mathrm{cm}^{3}$, which was the density of calcite. For Sample 1, the total mass of solid particles before the treatment was $64 \mathrm{mg} / \mathrm{L}$, which corresponded to $64 \mathrm{mg} / \mathrm{L} \mathrm{CaCO} \mathrm{Ca}_{3}$ hardness. The value increased to $104 \mathrm{mg} / \mathrm{L}$ after the plasma treatment, which means that the difference of a $40 \mathrm{mg} / \mathrm{L}$ of ionic content in water was transformed from the dissolved ionic states into solid content during the process. Aforementioned titration results from Fig. 4(a) showed that the calcium ion hardness in

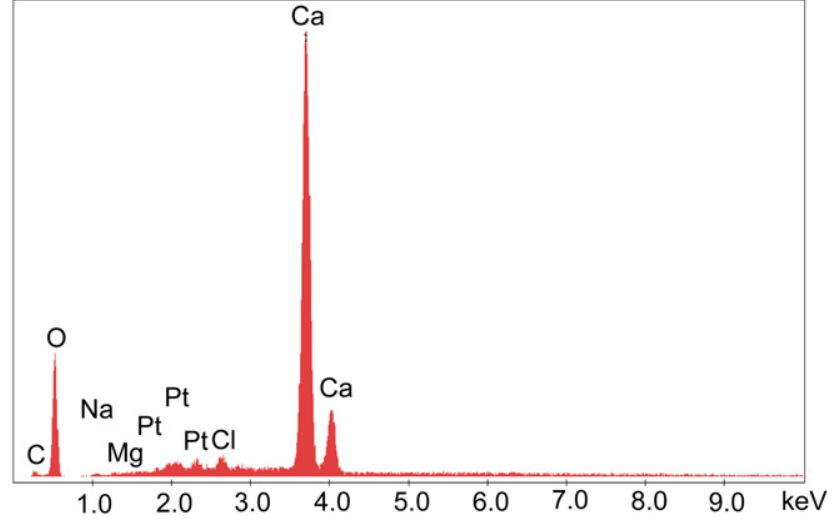

Fig. 8 - Elemental composition of the particles shown in Fig. 7(b) obtained by Energy Dispersion Spectrometer (EDS).

water was reduced by $25 \mathrm{mg} / \mathrm{L}$, equaling to $62.5 \mathrm{mg} / \mathrm{L}$ of calcium carbonate hardness. Considering that the resolution of the laser particle counter was $1 \mu \mathrm{m}$, which means that the precipitation of particles with less than 1- $\mu \mathrm{m}$-diameter was not taken into account, the results obtained by titration and laser particle counting were in good agreement, demonstrating that the calcium ions were transformed into calcium carbonate solids during the process. For all three cases, the results are summarized in Table 2 .

Fig. 7(a) shows the SEM photographs of particles retrieved from Sample 1 without the plasma treatment. Chemical composition of the particles was analyzed by Energy Dispersion Spectrometer (EDS). Fig. 8 shows that the particles were
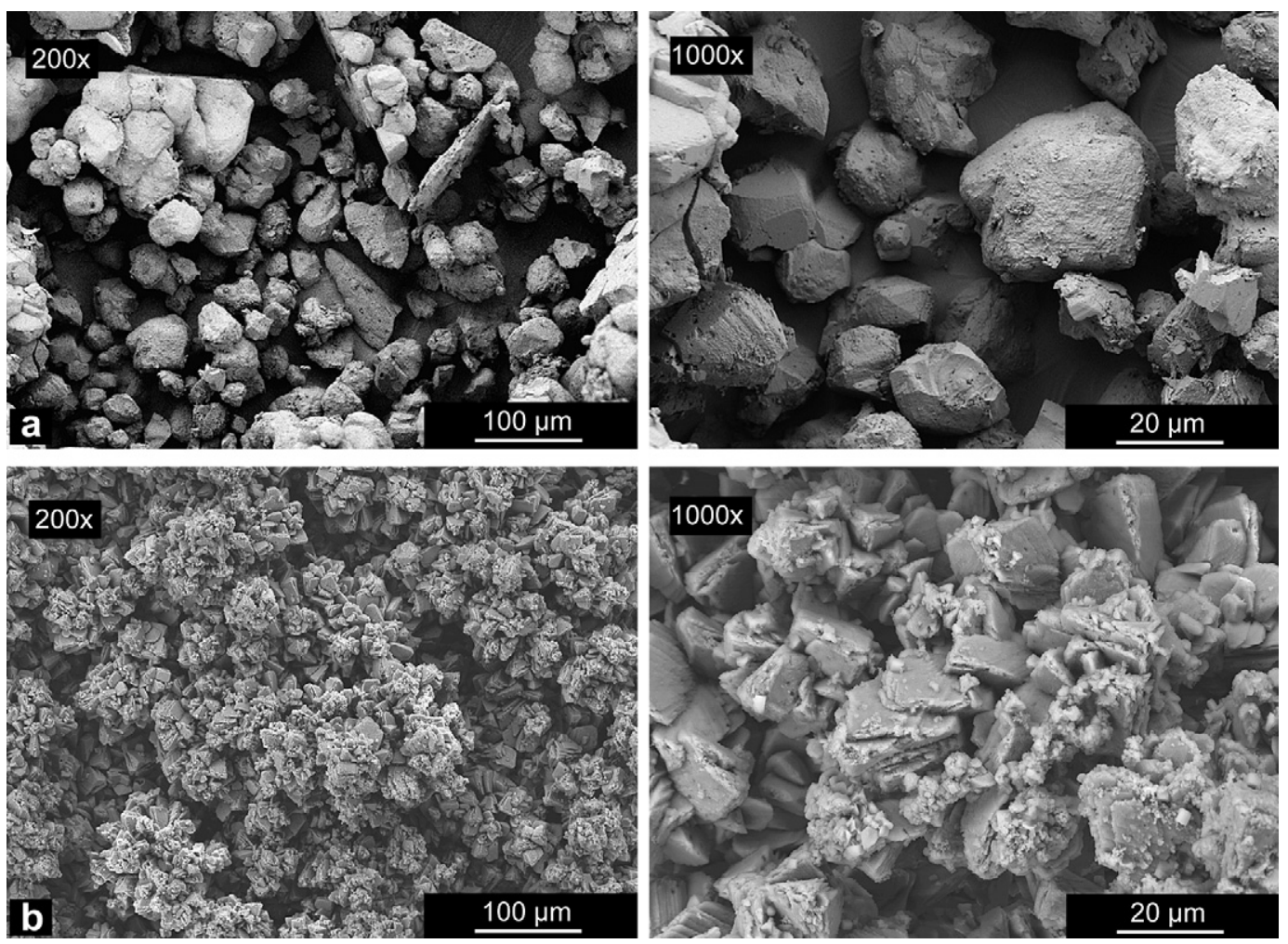

Fig. 7 - Comparison of SEM images of calcium carbonate crystals obtained from: (a) untreated water; (b) plasma treated water. 

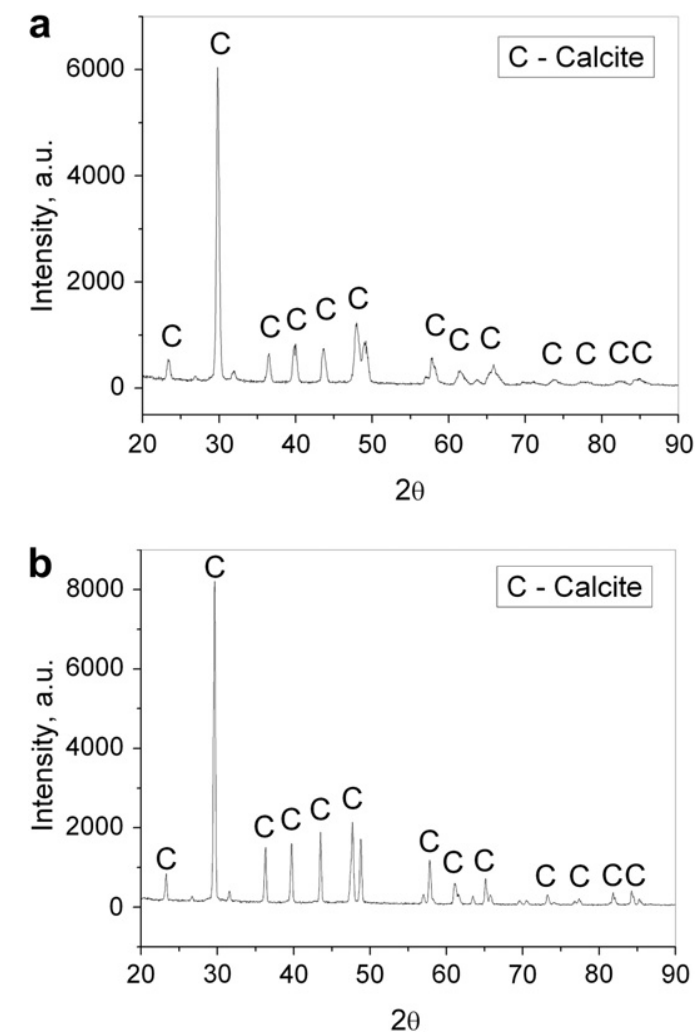

Fig. 9 - Comparison of XRD pattern of the particles calcium carbonate crystals obtained from: (a) untreated water; (b) plasma treated water.

mainly composed of calcium carbonate, with slight amounts of sodium and magnesium, as identified in Table 1, together with the platinum coated prior to the SEM observation. The crystals exhibited a morphology close to the rounded vaterite form. However X-ray diffraction pattern, as shown in Fig. 9(a) coincided with those of calcite. It was probably because of the thermodynamically unstable property of the vaterite crystals. The preparation of water samples usually took several days. Possibly during this period, vaterite might have recrystallized into more stable calcite crystals without changing shape.

Fig. 7(b) shows the photographs of the particles collected from Sample 1 after the plasma treatment. The crystals demonstrated the typical rhombohedron morphology of calcite. The formation of the calcite was confirmed by XRD shown in Fig. 9(b). The mean crystal size from the precipitation test was about $5 \mu \mathrm{m}$ after $20 \mathrm{~min}$ of the plasma treatment. For the treated water with high hardness (i.e. Samples 2 and 3), calcite crystals were also observed, and their sizes remained almost the same as that observed in Sample 1. The fact that the total amount of precipitates was increased as

Table 3 - Thermochemical data of Reactions 1-3.

\begin{tabular}{cccc} 
& Reaction 1 & Reaction 2 & Reaction 3 \\
\hline$\Delta H(\mathrm{~kJ} / \mathrm{mol})$ & 48.26 & -41.06 & 12.36 \\
$\Delta G(\mathrm{~kJ} / \mathrm{mol})$ & 43.60 & -20.9 & -47.70 \\
\hline
\end{tabular}

indicated by the laser particle counting suggests that the number of nuclei was significantly increased. That demonstrates that the plasma had induced chemical changes in the calcareous water during the treatment, which was implied later in the nucleation process during the precipitation test.

\section{Discussions}

This is the first time, to the authors' knowledge, to report the precipitation of dissolved calcium ions in water by the application of plasma discharge. In this section, the possible mechanism for the plasma-induced calcium carbonate precipitation is discussed.

First, the effect of electrolysis, which is another electricenergy-utilizing hard water softening technology, is examined. Gabrielli et al. (2006) studied the principle of softening process by electrolysis and showed that the precipitation was induced by a local $\mathrm{pH}$ increase near the vicinity of the cathode. A model electrolyser with an electrode area of $560 \mathrm{~cm}^{2}$ was tested. Under the operating current density of $20 \mathrm{~A} / \mathrm{m}^{2}$, applied voltage of $12 \mathrm{~V}$ and flow rate of $6 \mathrm{~L} / \mathrm{h}$, the calcium hardness of the water sample was reduced from 96 to about $72 \mathrm{mg} / \mathrm{L}$ when a quasisteady state operation was achieved. Hence the energy consumption was calculated as $806 \mathrm{~J} / \mathrm{L}$. For the present spark discharge, electrolysis most likely took place during the prebreakdown stage, as the current would be transferred by the ionized gas after the formation of the conductive channel between the two electrodes. The same level of the calcium hardness reduction was achieved as in Gabrielli's experiments after applying spark discharge in Sample 1 for 10 min, as shown in Fig. 4(a). The energy dissipation in the pre-breakdown stage was calculated as $984 \mathrm{~J} / \mathrm{L}$ based on the results shown in Fig. 3. However, it was difficult to estimate the exact amount of energy consumed by electrolysis, as multiple physical and chemical processes, including ionization, light emission, cavitation, shockwave and reactive species formation were initiated during the same stage, and it was likely that only a fraction of the $984 \mathrm{~J} / \mathrm{L}$ energy was dissipated in electrolysis. Another factor that may subdue the effect of electrolysis is the mixing of the liquid enhanced by the formation of shockwaves in the present study. Such a mixing may prohibit the formation of a local high $\mathrm{pH}$ area near the vicinity of the cathode. Therefore the electrolysis-induced precipitation might be suppressed in the present experiment, and it could be concluded that the reduction of water hardness may not be solely due to the electrolysis, though it may contribute partially to the precipitation process.

Next the chemistry behind the $\mathrm{CaCO}_{3}$ precipitation is described. The main reactions of the precipitation and dissolution of calcium carbonate in hard water system are (Snoeyink and Jenkins, 1982):

$\mathrm{Ca}^{2+}+\mathrm{CO}_{3}^{2-} \Leftrightarrow \mathrm{CaCO}_{3(\mathrm{~s})}$

and

$\mathrm{CaCO}_{3(\mathrm{~s})}+\mathrm{H}_{(\mathrm{aq})}^{+} \Leftrightarrow \mathrm{Ca}_{(\mathrm{aq})}^{2+}+\mathrm{HCO}_{3(\mathrm{aq})}^{-}$

In a saturated condition, the forward reaction of the precipitation of $\mathrm{CaCO}_{3}$ does not take place as both calcium and 
bicarbonate ions are hydrated. When water is supersaturated and sufficient energy is supplied, the water molecules are disturbed or become freed from the ions, resulting in the precipitation of $\mathrm{CaCO}_{3}$. Eq. (9) shows the dissolution of solid calcium carbonate by acid, a process that takes place during acid cleaning of scaled heat exchangers.

In reality, precipitation and dissociation reactions are much more complicated. The recombination and crystallization rates of calcium and carbonate ions are controlled by three reactions. Reaction 1 relates the dissociation of bicarbonate ions into the hydroxyl ions $\mathrm{OH}^{-}$and carbon dioxide (Muller-Steinhagen, 1999):

$\mathrm{HCO}_{3(\mathrm{aq})}^{-} \Leftrightarrow \mathrm{OH}_{(\mathrm{aq})}^{-}+\mathrm{CO}_{2(\mathrm{aq})} \quad$ (Reaction 1)

The forward reaction indicates the dissociation of the bicarbonate ions, which is the most critical step from the precipitation process. Of note is that the bicarbonate ions do not cause any harm in terms of scaling as long as they remain as bicarbonate ions. Reaction 1 shows the first step in the conversion of bicarbonate to carbonate ions. The presence of hydroxide ions is best indicated by a local increase in $\mathrm{pH}$, and carbon dioxide typically evolves from the water as gas over time.

In Reaction 2 hydroxyl ions produced from Reaction 1 further react with existing bicarbonate ions, producing carbonate ions and water (Muller-Steinhagen, 1999):

$$
\mathrm{HCO}_{3(\mathrm{aq})}^{-}+\mathrm{OH}_{(\mathrm{aq})}^{-} \Leftrightarrow \mathrm{H}_{2} \mathrm{O}_{(\mathrm{l})}+\mathrm{CO}_{3(\mathrm{aq})}^{2-} \quad \text { (Reaction 2) }
$$

Reaction 3 is the reaction between calcium and carbonate ions, resulting in the precipitation and crystallization of calcium carbonate particles (Muller-Steinhagen, 1999):

$\mathrm{Ca}_{(\mathrm{aq})}^{3+}+\mathrm{CO}_{3(\mathrm{aq})}^{2-} \Leftrightarrow \mathrm{CaCO}_{3(\mathrm{~s})} \quad$ (Reaction 3)

Table 3 presents the thermochemistry of the above three reactions (Snoeyink and Jenkins, 1982). The values of $\Delta H$ and $\Delta G$ give some useful insights into the behavior of the system of reactions. The endothermic Reaction 1 is the most ratelimiting, since it needs a relatively large input of energy to continue in the forward direction based on the high enthalpy value. Since the Gibbs free energy is relatively high, this reaction will tend to form bicarbonate ions, unless this energy restriction is overcome. In order for hydroxide ions to be produced as a result of the dissociation of the bicarbonate ions, the energy required for the reaction can be calculated as:

$$
\frac{48,260 \mathrm{~J}}{\mathrm{~mol}} \frac{1 \mathrm{~mol}}{6.02 \times 10^{23} \mathrm{ions}}=0.801 \times 10^{-19} \mathrm{~J}(\text { or } \sim 0.5 \mathrm{eV})
$$

To overcome the barrier, enough energy needs to be added to water so that the bicarbonate ions can be dissociated, subsequently precipitating dissolved calcium ions to $\mathrm{CaCO} 3$ via Reactions 2 and 3.

Thus, it is clear that the dissociation of bicarbonate ions plays an important role in the precipitation process, as observed in the present experiments (Fig. 5). Note that the reaction rate coefficient $k$ for Reaction 1 can be described as:

$k=\mathrm{A} e^{-E_{\mathrm{a}} / \mathrm{T}}$

where $A$ is a constant, $E_{\mathrm{a}}$ is activation energy for the reaction, $\mathrm{T}$ is the system temperature (in the unit of $\mathrm{eV}$ ). It is obvious that a high temperature will lead to a high precipitation rate. For example, when hard water is heated (i.e. volume heating) by boiling or microwave, the calcium carbonate deposit can be usually observed in bulk solution or heated surface. For plasma treatment cases, the total input energy was on the level on $\mathrm{kJ} / \mathrm{L}$, so that no significant volume heating could exist. However, direct pulsed spark discharges in water have been shown to generate a temperature up to 5000-10,000 K (about 0.5-1 eV) inside the plasma channel (Sunka et al., 1999), a phenomenon which is sufficient to induce a direct pyrolysis of bicarbonate ions. The high-temperature zone is highly localized around the plasma channel so that higher efficiency could be achieved over the volume heating under the same energy input level due to the exponential dependence of the reaction rate on the temperature. The maximum temperature depends on both plasma power and water properties. Furthermore, the thermal dissociation of bicarbonate ions may be enhanced by the emission of UV light from the hightemperature plasma channel, which functions as a blackbody radiation source. Full-spectrum UV radiation was demonstrated to be produced from spark discharge (Sunka, 2001). The VUV $(\lambda=75-185 \mathrm{~nm}$ ) emitted would be absorbed by the water layer immediately surrounding the plasma channel, leading to the expansion of the high-temperature zone (Locke et al., 2006). However, the amount of solution that can be treated directly by the thermal process is still limited by the small volume of the high-temperature zone.

Another factor that may contribute to the dissociation of bicarbonate ions is the electric field. There are reports about the increase of nucleation rate of different crystals when subjected to external electric fields ( $\mathrm{He}$ and Hopke, 1993; Chibowski et al., 1994; Katz et al., 1994). Dhanasekaran and Ramasamy (1986) studied the free energy required for the formation of a two-dimensional nucleus of any possible shape under electric field. They calculated the critical free energy for nucleation as.

$$
\Delta G=\frac{\beta^{2} \sigma^{2}}{4 k T\left(\ln \alpha+\varphi E^{2}\right)}
$$

where $\beta$ is a constant depending on the geometrical shape of the nucleus, $\sigma$ is the interfacial tension, $k$ is the Boltzmann constant, $\mathrm{T}$ is the temperature, $\alpha$ is the supersaturation ratio, $E$ is the external electric field, and $\varphi$ is defined as $-\epsilon_{0} v\left[\left(\left(1 / \epsilon_{\mathrm{s}}\right)-\left(1 / \epsilon_{1}\right)\right) \sin ^{2} \theta+\left(\epsilon_{\mathrm{s}}-\epsilon_{1}\right) \cos ^{2} \theta\right] / 8 \pi k T$, where $v$ is the volume of the crystalline nucleus, $\theta$ is the angle between the direction of the electric field and the nucleation surface, and $\epsilon_{\mathrm{S}}$ and $\epsilon_{\mathrm{l}}$ are the dielectric constant of the nucleus and solution, respectively. According to Dhanasekaran and Ramasamy, the free energy for nucleation decreases as the strength of electric field increases at certain angles, which leads to a higher nucleation rate.

The effect of electric field could also be explained by the disruption of the electric double layer of hydrated ions. Dissolved calcium and bicarbonate ions do not react at room temperature as both ions are surrounded by water molecules forming electric double layers. A number of researchers postulated that the magnetic or electric field, if strong enough, might disrupt the electric double layer and initiate the precipitation (Gehr et al., 1995; Baker and Judd, 1996; 
Higashitani and Oshitan, 1997). In order for an electric field to directly affect the electric double layer near a negatively charged surface, one needs an electric field that provides force which can overcome the force in the electric double layer. Typically, there are approximately two electron volts across an electric double layer, and the Debye length for a dilute solution like water is about $10 \mathrm{~nm}$ (Levine, 1978; Probstein, 1989). Thus, the electric field required to directly affect the electric double layer becomes approximately $2 \mathrm{~V} /$ $10 \mathrm{~nm}=2 \times 10^{8} \mathrm{~V} / \mathrm{m}$. To initiate pulsed electric discharges in water using a point-to-plane electrode system, it is necessary to have a high intensity electric field at the tip of a point electrode, which can be calculated as.

$E \approx \frac{\mathrm{V}}{r_{\mathrm{e}}}$

where $\mathrm{V}$ is the applied voltage, and $r_{\mathrm{e}}$ is the radius of curvature at the tip of the electrode. For the present system, $\mathrm{V}=24 \mathrm{kV}$ and $r_{\mathrm{e}} \approx 200 \mu \mathrm{m}$, leading to an electric field of $1.2 \times 10^{8} \mathrm{~V} / \mathrm{m}$, which is comparable to the electric field required to disrupt the hydration shells of the ions, and could possibly accelerate the dehydration process of ionic pair association. Additionally, unlike the highly localized thermal effect, the electric effect is not limited to the vicinity of the electrode tip. After the initiation of a pulsed discharge, multichannel streamers with lengths up to several centimeters could be produced when propagating from one electrode to the other electrode. An et al. (2007) measured the radius of the streamers and inferred that the electric field at the tip of the self-propagating streamers was more than $2 \times 10^{9} \mathrm{~V} / \mathrm{m}$. Subsequently, the bicarbonate ions may be dissociated along the propagation path of the streamers, leading to the precipitation of calcium carbonate in bulk water as described earlier.

For the different saturation levels in different cases, it was hypothesized that it could be related to the localization of the beneficial effect of the plasma (electric field, heat, electrolysis), and the acidification of the solution by the plasma as described in Eqs. (5) and (6), which was detrimental to the precipitation. Dynamic equilibrium was possibly reached at different levels between the dissociation of $\mathrm{HCO}_{3}^{-}$, the rate of which depended on the property of the solution for different cases, and the production of $\mathrm{H}^{+}$, which was largely decided by the input energy.

Finally, the anticipated benefits of the current work are discussed from the economic point of view. For a modern 1000-MW fossil-fueled power plant with $40 \%$ efficiency, it would reject $1500 \mathrm{MW}$ of heat at full load, using about $2800 \mathrm{~m}^{3} /$ min of circulating water based on $10^{\circ} \mathrm{C}$ temperature difference in a condenser (El-Wakil, 1984). As heat is removed via the evaporation of pure water at cooling tower, the need for makeup water is about $28 \mathrm{~m}^{3} / \mathrm{min}$ to compensate the water loss through evaporation, wind drift and blowdown (El-Wakil, 1984). Assuming that the makeup water is moderately hard with a $\mathrm{CaCO}_{3}$ hardness of $100 \mathrm{mg} / \mathrm{L}$, the total amount of $\mathrm{CaCO}_{3}$ it brings into the cooling tower is:

$\dot{\mathrm{m}}_{\mathrm{CaCO}_{3}}=28 \mathrm{~m}^{3} / \mathrm{min} \times 100 \mathrm{mg} / \mathrm{L}=2800 \mathrm{~g} / \mathrm{min}$

In a typical cooling tower application, the COC is usually maintained at 3.5. That means the hardness in the circulating cooling water is maintained at approximately $350 \mathrm{mg} / \mathrm{L}$, and the blowdown rate can be calculated, if the wind drift loss is neglected, as:

$q_{\text {blowdown }}=\frac{q_{\text {makeup }}}{\text { COC }}=8 \mathrm{~m}^{3} / \mathrm{min}$

It has been demonstrated that the present pulsed plasma technology can continuously precipitate $\mathrm{Ca}^{2+}$ from water, and potentially allows a cooling tower to operate at a higher COC than the current standard due to the reduction in blowdown frequency. Ideally, zero blowdown operation could be achieved, if all the calcium ions brought into the cooling tower by the makeup water are precipitated by the plasma, so that the constant mineral concentration is maintained in the main cooling loop without blowdown. The energy cost, as shown earlier, is about $1800 \mathrm{~J} / \mathrm{L}$ to achieve an average $25 \%$ reduction in water hardness. Assume that the cooling water is treated in a side-stream loop, the flow rate needed is:

$q=\frac{\dot{m}_{\mathrm{CaCO}_{3}}}{350 \mathrm{mg} / \mathrm{L} \times 25 \%}=32 \mathrm{~m}^{3} / \mathrm{min}$

which is approximately $1.2 \%$ of the flow rate of the main loop. The power needed to treat the water in the side-stream loop for the $25 \%$ reduction in the water hardness can be calculated as:

$P=\frac{1800 \mathrm{~J} / \mathrm{L} \times 32 \times 10^{3} \mathrm{~L} / \mathrm{min}}{60 \mathrm{~s} / \mathrm{min}}=960 \mathrm{~kW}$

which is $0.1 \%$ of the full capacity of the $1000-\mathrm{MW}$ power plant. In the mean while, the flow rate of the makeup water can be reduced from $28 \mathrm{~m}^{3} / \mathrm{min}$ to $20 \mathrm{~m}^{3} / \mathrm{min}$, which equals a saving of approximately $11,500 \mathrm{~m}^{3}$ per day, due to the elimination of blowdown.

\section{Conclusion}

The present study investigated the effect of pulsed spark discharge in water on the precipitation of dissolved calcium ions. Tests were conducted at different hardness levels using supersaturated hard water prepared from a laboratory cooling tower. After 10-min plasma treatment with an energy input of $1800 \mathrm{~J} / \mathrm{L}$, calcium hardness dropped by 20-26\%, comparing with zero reduction for untreated cases. The precipitation of calcium ions was further confirmed by bicarbonate ion measurement and laser particle counting. Both SEM images and XRD analysis showed smaller and crystallized calcite with rhombohedron morphology for the treated cases compared to a round structure observed for the no-treatment cases. The comparison of energy consumption by electrolysis and plasma was made, and it was concluded that the electrolysis might be partially responsible for the reduction of the water hardness. It was also hypothesized that both the intense local heating and high electric field, which may affect the organization of water molecules in the electric double layer of hydrated ions, contribute to the precipitation process.

Additional experiments were performed recently to examine the effect of different factors, including UV radiation, local heating, formation of chemical species and electric field, 
on the precipitation process. Details of the experiments will be reported soon.

\section{Acknowledgement}

This work was supported by U.S. Department of Energy, National Energy Technology Laboratory, through contract DE-NT0005308.

\section{R E F E R E N C E S}

Akiyama, H., 2000. Streamer discharges in liquids and their applications. IEEE Trans. Dielectr. Electr. Insul. 7, 646-653.

An, W., Baumung, K., et al., 2007. Underwater streamer propagation analyzed from detailed measurements of pressure release. J. Appl. Phys. 101, 053302.

Baker, J.S., Judd, S.J., 1996. Magnetic amelioration of scale formation. Water Res. 30, 247-260.

Chibowski, E., Holysz, L., et al., 1994. Changes in zeta potential and surface free energy of calcium carbonate due to exposure to radiofrequency electric field. Colloids Surf. A: Physicochem. Eng. Asp. 92, 79-85.

Cho, Y.I., Fridman, A.F., et al., 2004. Physical water treatment for fouling prevention in heat exchangers. Adv. Heat Transf. 38, 1-72.

Cho, Y.I., Lee, S.H., et al., 2003. Physical water treatment for the mitigation of mineral fouling in cooling-water applications. ASHRAE Trans. 109, 346-357.

Coetzee, P.P., Yacoby, M., et al., 1998. Scale reduction and scale modification effects induced by $\mathrm{Zn}$ and other metal species in physical water treatment. Water SA 24, 77-84.

Dhanasekaran, R., Ramasamy, P., 1986. Two-dimensional nucleation in the presence of an electric field. J. Cryst. Growth 79, 993-996.

El-Wakil, M.M., 1984. Powerplant Technology. McGraw-Hill, New York.

Fathi, A., Mohamed, T., et al., 2006. Effect of a magnetic water treatment on homogeneous and heterogeneous precipitation of calcium carbonate. Water Res. 40, 1941-1950.

Feeley, T.J., Skone, T.J., et al., 2008. Water: a critical resource in the thermoelectric power industry. Energy 33, 1-11.
Gabrielli, C., Maurin, G., et al., 2006. Electrochemical water softening: principle and application. Desalination 206, 150-163.

Gehr, R., Zhai, Z.A., et al., 1995. Reduction of soluble mineral concentration in $\mathrm{CaSO}_{4}$ saturated water using a magnetic field. Water Res. 29, 933-940.

He, F., Hopke, P.K., 1993. Experimental study of ion-induced nucleation by radon decay. J. Chem. Phys. 99, 9972-9978.

Higashitani, K., Oshitan, J., 1997. Measurements of magnetic effects on electrolyte solution by atomic force microscopy. Process Saf Environ Protect 75, 115-119.

Katz, J.L., Fisk, J.A., et al., 1994. Condensation of a supersaturated vapor IX. Nucleation on ions. J. Chem. Phys. 101, 2309-2318.

Lee, G.J., Tijing, L.D., et al., 2006. Use of catalytic materials for the mitigation of mineral fouling. Int. Commun. Heat Mass Transf. 33, 14-23.

Levine, I.N., 1978. Physical Chemistry. McGraw-Hill, New York.

Locke, B.R., Sato, M., et al., 2006. Electrohydraulic discharge and nonthermal plasma for water treatment. Ind. Eng. Chem. Res. 45, 882-905.

Muller-Steinhagen, H., 1999. Cooling water fouling in heat exchangers. Adv. Heat Transf. 33, 415-496.

Muller-Steinhagen, H., 2000. Handbook of Heat Exchanger Fouling - Mitigation and Cleaning Technologies. Publico Publications, Germany.

Panchal, C.B., Knudsen, J.G., 1998. Mitigation of water fouling: technology status and challenges. Adv. Heat Transf. 31, 431-474.

Probstein, R.F., 1989. Physicochemical Hydrodynamics. Butterworths, Boston.

Radler, S., Ousko-Oberhoffer, U., 2005. Optimised heat exchanger management-achieving financial and environmental targets. In: Sixth International Conference on Heat Exchanger Fouling and Cleaning, Irsee, Germany.

Snoeyink, V.L., Jenkins, D., 1982. Water Chemistry. Wiley, New York.

Somerscales, E.F.C., 1990. Fouling of heat exchangers: an historical review. Heat Transf. Eng. 11, 19-36.

Sunka, P., 2001. Pulse electrical discharges in water and their applications. Phys. Plasmas 8, 2587-2594.

Sunka, P., Babicky, V., et al., 1999. Generation of chemically active species by electrical discharges in water. Plasma Sources Sci. Technol. 8, 258-265.

Xiaokai, X., 2008. Research on the electromagnetic anti-fouling technology for heat transfer enhancement. Appl. Therm. Eng. 28, 889-894. 


\section{Mineral Fouling Control by Underwater Plasma Discharge in a Heat Exchanger}

\section{Yong Yang \\ e-mail: yy65@drexel.edu}

\section{Hyoungsup Kim}

e-mail: hk378@drexel.edu

\section{Alexander Fridman \\ e-mail: fridman@drexel.edu}

\section{Young I. Cho}

e-mail: choyi@drexel.edu

Department of Mechanical Engineering and Mechanics, Drexel University, Philadelphia, PA 19104

The excessive mineral contents in water circulation systems could cause severe fouling in heat transfer equipment. The present study investigated the effect of underwater pulsed spark discharges on the mitigation of mineral fouling in a concentric counterflow heat exchanger. Artificial hard water with calcium carbonate hardness of $250 \mathrm{mg} / \mathrm{L}$ was used with velocity ranging from $0.1 \mathrm{~m} / \mathrm{s}$ to 0.5 $\mathrm{m} / \mathrm{s}$ and zero blowdown. Fouling resistances decreased by 50 $72 \%$ for the plasma treated cases compared with the values for no-treatment cases, indicating that the pulsed spark discharge could significantly mitigate the mineral fouling on the heat exchanger surface. [DOI: 10.1115/1.4003116]

Keywords: physical water treatment, plasma, heat exchanger, mineral fouling

\section{Introduction}

Calcium carbonate is one of the most common scale-forming minerals occurring in heat exchangers using cooling water. It is generally the first mineral to precipitate out when water is heated due to its inverse solubility. Thus, the control of calcium carbonate scale is often the limiting factor in most industrial cooling water applications, as the scale decreases the efficiency of heat exchangers because of its insulating effect.

Physical water treatment (PWT) is a nonchemical method to mitigate mineral fouling. A number of studies have been reported for the effectiveness of solenoid coils, permanent magnets, and catalytic alloys [1-5]. Recently Yang et al. [6] reported that underwater pulsed spark discharge may induce the precipitation of calcium carbonate in supersaturated water. Note that the precipitation of dissolved mineral ions takes place in the bulk water with PWT instead of on the heat exchanger surfaces. This is the key process for all PWT methods, as the particles suspended in water tend to form a soft coating on heat transfer surfaces. If the shear force produced by flow is large enough to remove the soft coating, mineral fouling can be prevented or mitigated.

In this paper, direct pulsed spark discharge generated in water was used to mitigate mineral fouling in a double-pipe heat exchanger. A new method using microsecond-duration pulsed

Contributed by the Heat Transfer Division of ASME for publication in the JourNAL OF HeAT TRANSFER. Manuscript received June 26, 2010; final manuscript received November 19, 2010; published online February 1, 2011. Assoc. Editor: Sujoy Kumar Saha plasma in water is a major improvement over the aforementioned PWTs because these previous PWTs produce relatively weak induced electric fields in water, where the field strength is often $\sim 1 \mathrm{mV} / \mathrm{cm}$ or less due to involved physics laws such as Faraday's law. In comparison, an electric field above $10^{6} \mathrm{~V} / \mathrm{cm}$ in water could be produced by pulsed plasma in water, leading to a higher efficiency than previous PWT methods.

\section{Experimental Methods}

The present study conducted fouling experiments in a heat exchanger by circulating artificially prepared hard water through a simulated cooling tower system. Figure 1 shows the schematic of the present test facility. The main component of the apparatus was a counterflow concentric heat exchanger. The length of the heat transfer test section was $600 \mathrm{~mm}$. Hot water was circulated inside the inner copper tube at a constant velocity of $1.0 \mathrm{~m} / \mathrm{s}$, while cold hard water was circulated in the opposite direction through the annulus gap between the inner and outer tubes at two different velocities $(0.1 \mathrm{~m} / \mathrm{s}$ and $0.5 \mathrm{~m} / \mathrm{s})$. Details of the heat exchanger were described elsewhere [7]. The initial $\mathrm{CaCO}_{3}$ hardness of the cold water was $250 \mathrm{mg} / \mathrm{L}$, which was obtained by mixing $\mathrm{CaCl}_{2}$ and $\mathrm{NaHCO}_{3}$ at right proportions. The final hardness at the end of fouling tests was approximately $600-700 \mathrm{mg} / \mathrm{L}$ as zero blowdown was used in the tests. The inlet temperature of cold water was maintained at $20 \pm 3^{\circ} \mathrm{C}$, whereas the inlet temperature of hot water was kept at $95 \pm 3^{\circ} \mathrm{C}$ using a hot water heater. Four copperConstantan (type $\mathrm{T}$ ) thermocouples were installed at the inlets and outlets of the counterflow heat exchanger and measured temperatures every $1 \mathrm{~min}$ for a test period of 24-48 h.

The heat transfer rate $Q$ was calculated from both hot- and cooling water sides as

$$
Q=\dot{m}_{h} c_{p} \Delta T_{h}=\dot{m}_{c} c_{p} \Delta T_{c}
$$

where $\dot{m}_{h}$ and $\dot{m}_{c}$ are the mass flow rates of hot and cooling water, respectively; $c_{p}$ is the specific heat of water; and $\Delta T_{h}$ and $\Delta T_{c}$ are the temperature differences between inlet and outlet of hot and cooling water, respectively. The measured heat transfer rate at the hot water side was approximately 5\% larger than that at the cold water side as parasitic heat loss from the outer tube wall to the surroundings. Here the heat transfer rate measured from the cooling water side was used. The value of $Q$ varied from $1.9 \mathrm{~kW}$ to 3.2 $\mathrm{kW}$ depending on the flow velocity at the cold water side.

The overall heat transfer coefficient $U$ was calculated as [8]

$$
U=\frac{Q_{c}}{A \Delta T_{\mathrm{LMTD}}}
$$

where $A$ is the heat transfer surface area, and $\Delta T_{\mathrm{LMTD}}$ is the logmean-temperature-difference. $\Delta T_{\mathrm{LMTD}}$ was determined as [8]

$$
\Delta T_{\mathrm{LMTD}}=\frac{\left(T_{h, o}-T_{c, i}\right)-\left(T_{h, i}-T_{c, o}\right)}{\ln \left[\frac{\left(T_{h, o}-T_{c, i}\right)}{\left(T_{h, i}-T_{c, o}\right)}\right]}
$$

The fouling resistance $R_{f}$ was calculated as [8]

$$
R_{f}=\frac{1}{U_{f}}-\frac{1}{U_{\text {is }}}
$$

where $U_{f}$ is the overall heat transfer coefficient for fouled states, and $U_{\text {is }}$ is the overall heat transfer coefficient at the initial state. The latter $\left(U_{\text {is }}\right)$ was determined using distilled water during the initial calibration run prior to the fouling tests.

The spark discharge generation system consisted of two components: a discharge chamber utilizing a point-to-plain electrode system immersed in water and a high-voltage power supply with a capacitive energy storage. In a typical experiment, an $8.5 \mathrm{nF}$ capacitor bank stored energy at a voltage of $25 \mathrm{kV}$ was discharged 


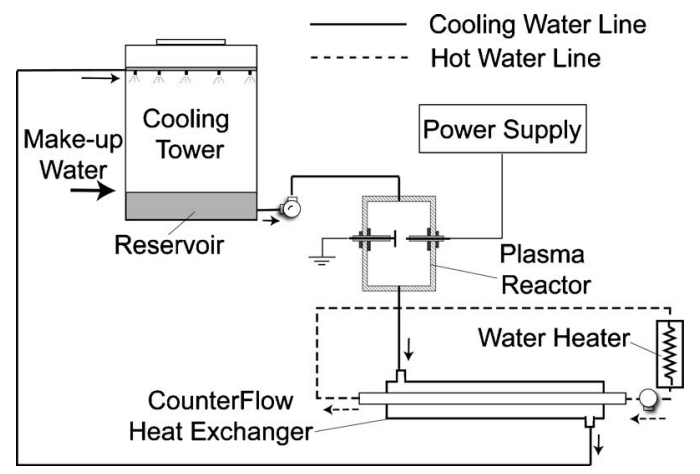

Fig. 1 Schematic of the present experimental setup

through a $5 \mathrm{~mm}$ electrode gap into the discharge chamber. The peak current of these pulses was about 90 A. Details of the circuit system were discussed elsewhere [9].

Average scale thickness was estimated by the following equation:

$$
D=\frac{m_{f}-m_{0}}{\rho_{c} A}
$$

where $m_{0}$ and $m_{f}$ are the masses of the copper tube before and after fouling tests, respectively, and $\rho_{c}$ is the density of calcite $\left(2.7 \mathrm{~g} / \mathrm{cm}^{3}\right)$. Scale adhesion was tested by the taping method [10]. First two cuts are made into the scale coating with a $90 \mathrm{deg}$ intersection. Then a pressure sensitive tape (Scotch ATG 928, 3M Inc., St. Paul, MN) was applied on the center of the intersection at ten randomly selected points on the surface of the fouled tubes and then pulled off to determine the coating adhesion stress. This technique produced only qualitative results, and completely lifting, partial lifting, and complete adhesion were identified. Scanning electron microscopy (SEM) (FEI XL30) images were obtained from the fouled copper tubes at Drexel SEM Laboratory (Philadelphia, PA).

\section{Results and Discussions}

The scale deposition involved the cumulative effect of a direct diffusion of dissolved calcium ions to a heat transfer surface and the removal of scale deposits from the surface. Figure 2 shows the results for the fouling tests obtained for the no-treatment and plasma treated cases at a flow velocity of $0.1 \mathrm{~m} / \mathrm{s}$. The fouling resistance in the no-treatment case demonstrated a slow increase in the first $14 \mathrm{~h}$ of operation. At $t=15 \mathrm{~h}$, the fouling resistance increased dramatically as the entire surface of the copper tube was fully covered by mineral scales. After that, the fouling resistance began to slowly rise as the thickness of the scale layer gradually increased until the end of test, indicating that the deposition rate

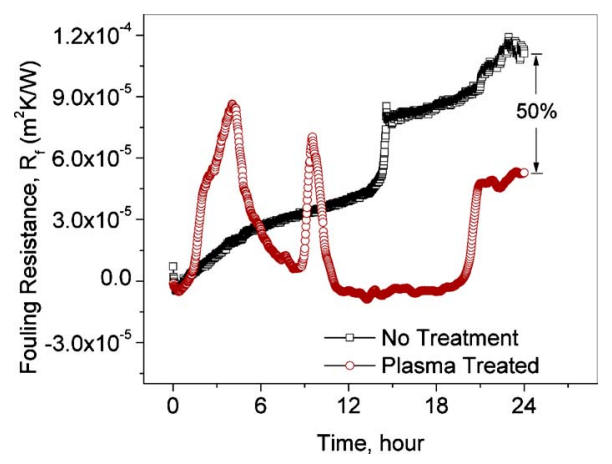

Fig. 2 Fouling resistances over time for no-treatment and plasma treated cases with a flow velocity of $0.1 \mathrm{~m} / \mathrm{s}$
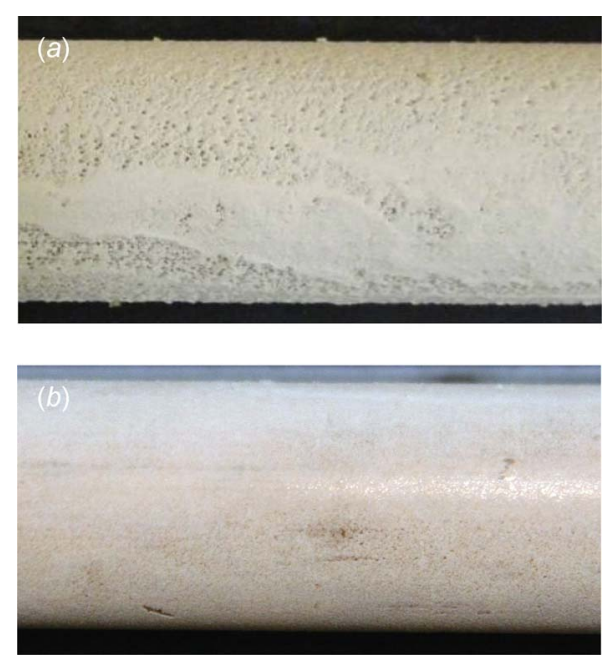

Fig. 3 Photographic images of scales for (a) no-treatment and (b) plasma treatment cases at a flow velocity of $0.1 \mathrm{~m} / \mathrm{s}$

of the scales was consistently larger than the removal rate during this period because of the low flow velocity. Note that under normal conditions, $U_{f}$ will drop as the scale grows so that $R$ will always be positive. However, when the wall of the heat transfer tube becomes rough particularly at the beginning of fouling tests, the convective heat transfer coefficient increases beyond that from the clean tube condition. Hence one can have negative fouling resistances especially at the beginning of test.

The fouling resistance obtained in the plasma treatment case depicted a completely different trend. The fouling resistance had a steep increase to a maximum value in the first $4 \mathrm{~h}$ of operation. Note that there are two different categories of fouling: particulate fouling and precipitation fouling. The former refers to the deposition of suspended particles to the heat transfer surface in the form of soft sludge. This type of fouling can easily be removed by shear forces created by flow than those deposits produced from the precipitation of mineral ions directly on the heat transfer surface, i.e., precipitation fouling [11]. It was demonstrated in our previous study that the precipitation of calcium carbonate could be induced by pulsed spark discharge in supersaturated hard water and thus creating a significantly greater number of $\mathrm{CaCO}_{3}$ particles than the untreated water [6]. Hence, much faster particulate fouling must have taken place at the first several hours of the test, causing the dramatic increase in the fouling resistance. At $t$ $=4 \mathrm{~h}$, the fouling resistance showed a significant drop, indicating that large scale pieces were dislodged due to the shear stress of the water flow. A similar process of particulate fouling buildup and dislodge was repeated during the period between $9 \mathrm{~h}$ and $12 \mathrm{~h}$. The final asymptotic fouling resistance at the end of the test was $50 \%$ lower than that obtained from the baseline test, clearly indicating the beneficial effect of the plasma discharge on the mitigation of mineral fouling.

Figure 3 shows photographs of sections of fouled copper tubes for both the no-treatment and plasma treated cases. Scale with about $1 \mathrm{~mm}$ average thickness was observed for the no-treatment case. For the case of the plasma treatment, the scale was about 0.4 $\mathrm{mm}$ thick, and the copper tone color of the copper tube was visible at the end of the fouling test.

Figure 4 shows the results for the fouling tests obtained at a flow velocity of $0.5 \mathrm{~m} / \mathrm{s}$. The fouling resistances at $0.5 \mathrm{~m} / \mathrm{s}$ showed significant drops compared with those obtained at $0.1 \mathrm{~m} / \mathrm{s}$, mainly because of the higher removal rate caused by the higher flow velocity. The fouling resistance curves obtained in the case for plasma treatment depicted a similar trend as the $0.1 \mathrm{~m} / \mathrm{s}$ case shown in Fig. 2 at the first $20 \mathrm{~h}$ period. The fast rise and fall of fouling resistance observed in the first $6 \mathrm{~h}$ period indicated both 


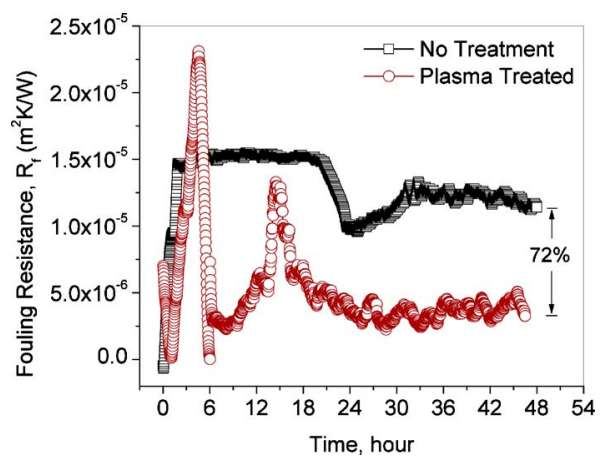

Fig. 4 Fouling resistances over time for no-treatment and plasma treated cases at a flow velocity of $0.5 \mathrm{~m} / \mathrm{s}$

accumulation and removal of large scale deposits on the heat exchanger surface. After $t=20 \mathrm{~h}$, the fouling resistance slightly went up and down numerous times until the end of the test, indicating that the old scales were repeatedly removed from the heat transfer surface as the new scales continued to develop. The final fouling resistance was reduced by $72 \%$ compared to that for the no-treatment case.

Figures 5(a) and 5(b) show SEM images of $\mathrm{CaCO}_{3}$ scales for both the no-treatment and plasma treated cases at a flow velocity of $0.5 \mathrm{~m} / \mathrm{s}$. The SEM images for the no-treatment case showed particles less than $10 \mu \mathrm{m}$ in size, with sharp crystal structures as a result of crystallization fouling. The images obtained with plasma treatment showed particles with a rounded shape and irregular sizes, probably caused by agglomeration of the precipitated particles produced by spark discharges. Note that the hardened scale caused by crystallization fouling has a stronger adhesion force to the surface and cannot be removed by brush punching, and thus acid cleaning is often required, while the scale formed through particulate fouling is usually soft and can be removed by the shear force of water flow [11]. The Scotch tape test results confirmed the hypothesis, as shown in Fig. 6. Complete lifting was observed for the case of plasma treatment, while the no-treatment case demonstrated zero or partial lifting. The difference of the scale adhesion force may explain why the underwater spark discharge was effective in the mitigation of mineral fouling on heat exchanger surfaces.

\section{Conclusions}

The present study investigated the effect of pulsed spark discharge in water on the mitigation of $\mathrm{CaCO}_{3}$ fouling in a concentric tube heat exchanger. Fouling tests were conducted with an initial water hardness of $250 \mathrm{mg} / \mathrm{L}$ at two different flow velocities $(0.1 \mathrm{~m} / \mathrm{s}$ and $0.5 \mathrm{~m} / \mathrm{s})$ with zero blowdown. The fouling resistances for the plasma treated cases dropped by 50-72\% compared
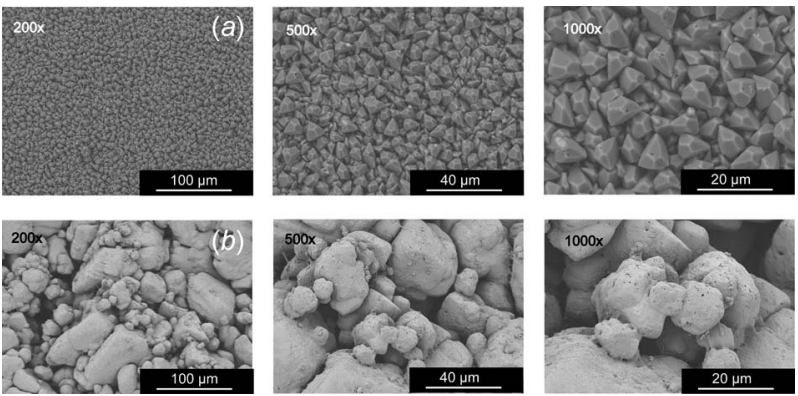

Fig. 5 SEM photographs of scales obtained for (a) notreatment and $(b)$ plasma treated cases at a flow velocity of 0.5 $\mathrm{m} / \mathrm{s}$

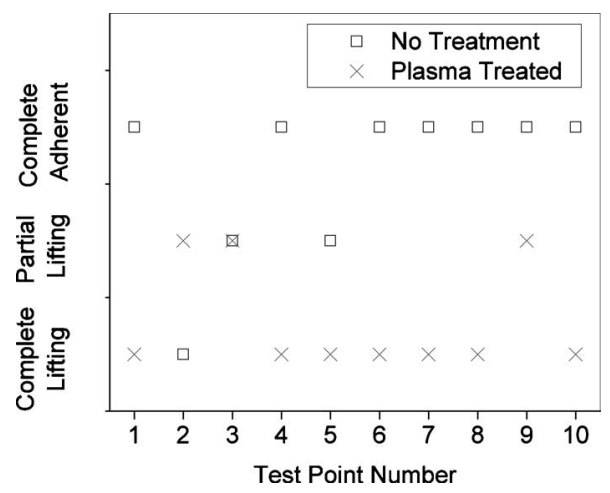

Fig. 6 Adhesion of scales obtained for no-treatment and plasma treated cases at a flow velocity of $0.5 \mathrm{~m} / \mathrm{s}$

with the no-treatment cases, depending on the flow velocity. The fouling resistance data confirmed that the pulsed spark discharge was beneficial in mitigating the mineral fouling in a heat exchanger by continuously producing suspended calcium carbonate particles in water. Subsequently, the suspended particles tended to form soft deposits on the surface of heat exchangers, which were removed by the shear force of water flow. SEM images showed larger and amorphous structures for the plasma treated cases, compared with the crystallized structures observed for the notreatment case.

\section{Acknowledgment}

This work was supported by the U.S. Department of Energy, National Energy Technology Laboratory, through Contract No. DE-NT0005308.

\section{Nomenclature}

$$
\begin{aligned}
A & =\text { area } \\
d & =\text { diameter } \\
D & =\text { scale thickness } \\
\dot{m} & =\text { mass flow rate } \\
Q & =\text { heat transfer rate } \\
R & =\text { fouling resistance } \\
t & =\text { time } \\
T & =\text { temperature } \\
U & =\text { heat transfer coefficient }
\end{aligned}
$$

Greek Symbols

$$
\rho=\text { density }
$$

\section{Subscripts}

$$
\begin{aligned}
c & =\text { condition on the cold water side } \\
f & =\text { fouled condition } \\
h & =\text { condition on the hot water side } \\
i & =\text { inlet } \\
o & =\text { outlet } \\
\text { is } & =\text { initial state } \\
\text { LMTD } & =\text { log-mean-temperature-difference }
\end{aligned}
$$

\section{References}

[1] Parsons, S. A., Judd, S. J., Stephenson, T., Udol, S., and Wang, B. L., 1997, "Magnetically Augmented Water Treatment," Process Saf. Environ. Prot., 75, pp. $98-104$

[2] Smith, C., Coetzee, P. P., and Meyer, J. P., 2003, "The Effectiveness of a Magnetic Physical Water Treatment Device on Scaling in Domestic Hot-Water Storage Tanks," Water SA, 29, pp. 231-236.

[3] Cho, Y. I., Lane, J., and Kim, W., 2005, "Pulsed-Power Treatment for Physical Water Treatment," Int. Commun. Heat Mass Transfer, 32, pp. 861-871.

[4] Lee, G. J., Tijing, L. D., Pak, B. C., Baek, B. J., and Cho, Y. I., 2006, "Use of Catalytic Materials for the Mitigation of Mineral Fouling," Int. Commun. Heat Mass Transfer, 33, pp. 14-23.

[5] Xiaokai, X., 2008, "Research on the Electromagnetic Anti-Fouling Technology 
for Heat Transfer Enhancement,” Appl. Therm. Eng., 28, pp. 889-894.

[6] Yang, Y., Kim, H., Starikovskiy, A., Fridman, A., and Cho, Y. I., 2010, “Application of Pulsed Spark Discharge for Calcium Carbonate Precipitation in Hard Water," Water Res., 44, pp. 3659-3668.

[7] Yang, Y., Kim, H., Fridman, A., and Cho, Y. I., 2010, "Effect of a PlasmaAssisted Self-Cleaning Filter on the Performance of PWT Coil for the Mitigation of Mineral Fouling in a Heat Exchanger," Int. J. Heat Mass Transfer, 53, pp. 412-422.

[8] Incropera, F. P., Dewitt, D. P., Bergman, T. L., and Lavine, A. S., 2007,
Fundamentals of Heat and Mass Transfer, Wiley, New York.

[9] Yang, Y., Gutsol, A., Fridman, A., and Cho, Y. I., 2009, "Removal of $\mathrm{CaCo}_{3}$ Scales on a Filter Membrane Using Plasma Discharge in Water," Int. J. Heat Mass Transfer, 52, pp. 4901-4906.

[10] Chalker, P. R., Bull, S. J., and Rickerby, D. S., 1991, "A Review of the Methods for the Evaluation of Coating-Substrate Adhesion," Mater. Sci. Eng., A, 140, pp. 583-592.

[11] Bott, T. R., 1995, The Fouling of Heat Exchangers, Elsevier Science, New York. 


\title{
Note: An underwater multi-channel plasma array for water sterilization
}

\author{
Y. Yang, ${ }^{1, a)}$ H. Kim, ${ }^{2}$ A. Starikovskiy, ${ }^{3}$ Y. I. Cho, ${ }^{2}$ and A. Fridman ${ }^{2}$ \\ ${ }^{1}$ College of Electrical and Electronics Engineering, Huazhong University of Science and \\ Technology, Wuhan 430074, China \\ ${ }^{2}$ Department of Mechanical Engineering and Mechanics, Drexel University, \\ Philadelphia, Pennsylvania 19104, USA \\ ${ }^{3}$ Department of Mechanical and Aerospace Engineering, Princeton University, \\ Princeton, New Jersey 08544, USA
}

(Received 17 May 2011; accepted 15 August 2011; published online 21 September 2011)

\begin{abstract}
A simple yet effective method to generate multi-channel plasma array in water is presented in this paper. Thin circular metal disks sandwiched between dielectric layers were used, allowing the production of large-volume underwater plasma array with higher stability. The system can be further scaled up by stacking multiple metal disks, making it suitable for large-scale industrial water treatment. Generation of UV and reactive species was identified by optical emission spectroscopy. Sterilization experiments were performed. Results show that the device was effective in deactivating $E$. coli in water over a wide range of initial concentrations ranging from $10^{4}$ to $10^{8} \mathrm{CFU} / \mathrm{ml}$. (c) 2011 American Institute of Physics. [doi:10.1063/1.3633945]
\end{abstract}

The increased presence of Escherichia coli (E. coli), along with various other bacteria in water has become a cause of global concern. In efforts to sterilize these bacteria, commercial applications, such as chlorine treatments, ultraviolet (UV) radiation, and ozone injection, have been implemented for potable water delivery systems. The use of underwater plasma discharges is a relatively new addition to the traditional methods. ${ }^{1-4}$ Plasma sterilization has been proved to be effective, safe, and environmental friendly in the deactivation of microorganisms in water. The synergistic effect of in situ production of radicals $(\mathrm{OH}, \mathrm{O}$, etc.), molecular species $\left(\mathrm{O}_{3}, \mathrm{H}_{2} \mathrm{O}_{2}\right.$, etc.), UV, electric field, and shockwaves has been shown to have higher energy efficiency than conventional oxidants and disinfectants.

To produce direct electric breakdown in water, a critical electric field of $10^{6} \mathrm{~V} / \mathrm{cm}$ is needed. Sharp point electrodes are usually used to provide the electric field enhancement. However, the stability of the plasma discharge is limited by the erosion at the point electrode tip, which will cause the decay of electric field at the tip and eventually lead to the extinction of the discharge. Furthermore, the scaling up of the plasma source still remains an issue, as the treatment zone is mostly limited at the vicinity of the point electrode. Different plasma sources were reported to improve the stability or scalability of plasma discharges in water, including wire-cylinder, pin-hole, and porous-ceramic-coated electrodes configuration, etc..$^{5-11}$ In this note, we demonstrate the disinfection of $E$. coli using a multi-channel plasma discharge array generated by a stacked circular disk electrode in water, which offers larger treatment volume, better controllability, and scalability than traditional point electrode systems.

Figure 1 shows a sketch of the present experiment setup. The high-voltage powered electrode consisted of circular stainless steel disks with a diameter of $100 \mathrm{~mm}$ and a thick-

\footnotetext{
a) Author to whom correspondence should be addressed. Electronic mail:
} yy65@drexel.edu. ness of $20 \mu \mathrm{m}$. The electric field at the edge of the stainless steel disk could be estimated as $E \sim 2 U / d$, where $U$ is the applied voltage and $d$ is the disk thickness. With a typical breakdown voltage of about $30 \mathrm{kV}$, electric field in the order of $10^{7} \mathrm{~V} / \mathrm{cm}$ can be achieved, which is comparable to that at the tip of a point electrode. The field strength would stay relatively constant throughout the discharge process as long as the thickness of the disk stays constant, thus eliminating the concern of the decay of electric field due to the erosion of the point electrode. ${ }^{12,13}$ The stainless steel disks were sandwiched between pairs of acrylic disks with a diameter of $105 \mathrm{~mm}$. The diameter of the acryl disk was slightly greater than that of the stainless steel disk so that when high voltage was applied on the stainless steel disk, the ionic prebreakdown current was limited to a small area enclosed by the acrylic disks and the edge of the stainless steel disk. Limiting the current to the small area allowed water to be heated and vaporized through joule heating, thus effectively promoting the initiation of the plasma discharges in these vaporized areas. The mechanism is similar to that of the pin-hole discharge (also known as diaphragm discharge) in water. ${ }^{14}$ However, diaphragm discharge is usually produced through a small hole with diameter ranging from $0.1 \mathrm{~mm}$ to $1 \mathrm{~mm}$, leading to very limited treatment capacity. The current design allows the generation of plasma along the periphery of the disk, resulting in a much larger treatment volume. Furthermore, the entire electrode system can be easily scaled up by stacking multiple metal disks for large-volume water treatment.

The pulsed power system consisted of a high-voltage DC generator, a capacitive energy storage, and a spark-gap based switch. The DC generator charged the 8.5-nF capacitor bank until the pulse was triggered by the air-filled spark gap switch, delivering the energy stored in the capacitor bank to water to create the breakdown. Figure 2 shows the typical voltage and current waveforms of the discharge. The initial steep rise in the voltage profile indicates the moment of breakdown in the spark gap. A peak voltage of $32 \mathrm{kV}$ was applied, and the 


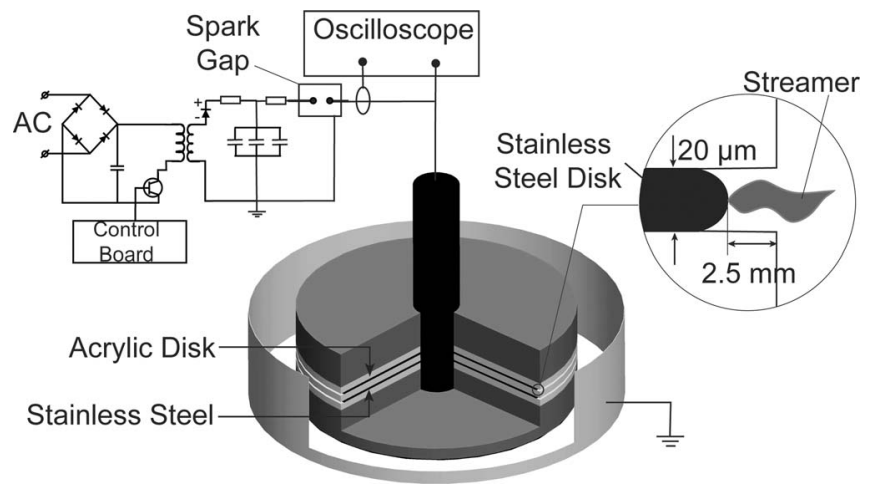

FIG. 1. Schematic diagram of the experiment setup.

full width at half maximum (FWHM) of the voltage signal was about $3 \mu \mathrm{s}$. Relative high pre-breakdown current ( $\sim 20 \mathrm{~A})$ was observed prior to the major breakdown stage due to the conductivity of water. After that, there was a sudden increase of the current to $120 \mathrm{~A}$ in about $1.5 \mu \mathrm{s}$, indicating the onset of the major breakdown.

Figures 3(a) and 3(b) show the images of the single-layer and double-layer plasma array generated with one and two stainless steel disks, respectively. The discharge array consisted of numerous channels, which were relatively evenly distributed along the edge of the stainless steel disks and formed a steady pattern. This was probably due to the interaction of repulsive Coulomb force and attractive Lorentz force between nearby discharges within the same voltage cycle. This distribution of the streamers bears similarity to phenomena observed in dielectric barrier discharges (DBD), where DBD streamers tend to form self-organized patterns. ${ }^{15}$ The breakdown process could be explained by the aforementioned bubble mechanism, in which the pre-breakdown current leads to the formation of low density regions, or "bubbles," near the electrodes through joule heating. After that major breakdown originates and develops via electron avalanches in these low density regions and forms the discharge channels.

Figure 4 shows measured optical emission spectra from the discharge array in distilled water. The spectra indicated combinations of a continuum radiation and strong atomic line emissions due to the presence of hydrogen and oxygen radicals from the decomposition of water. The most intense peak

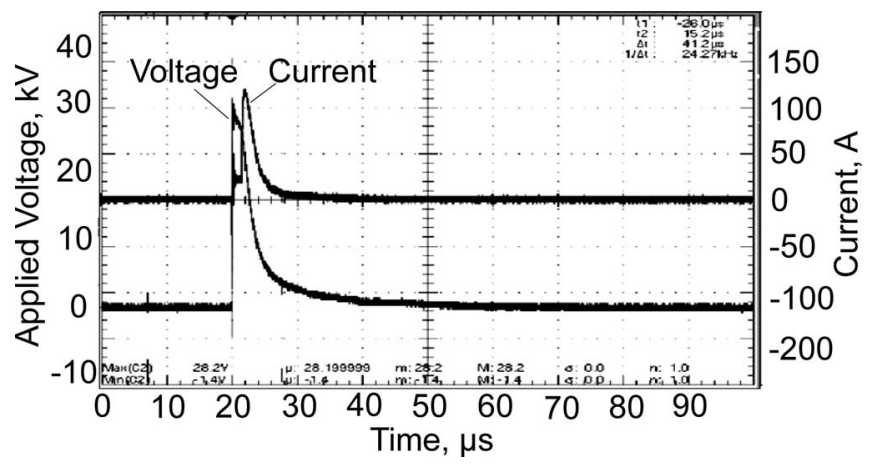

FIG. 2. Typical voltage and current waveform for the double-layer plasma array in water.

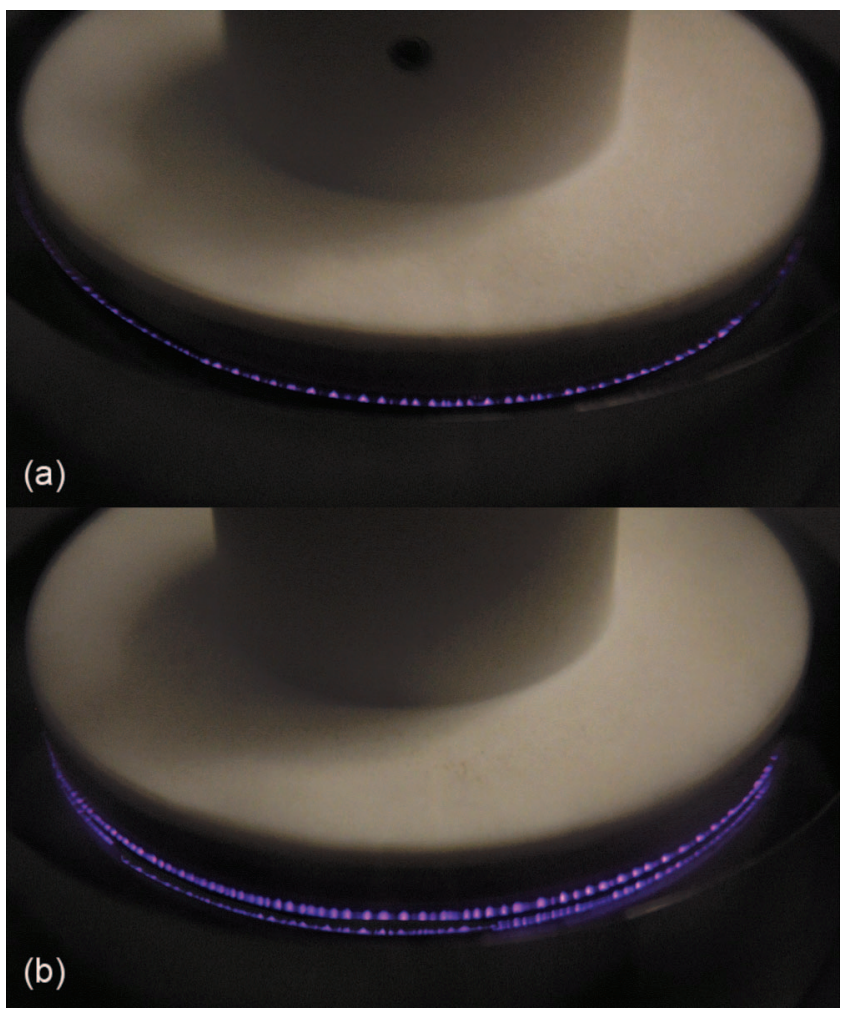

FIG. 3. (Color online) Image of the plasma array in water: (a) single layer; (b) double layer.

was the hydrogen Balmer series alpha line $\left(\mathrm{H}_{\alpha}\right)$ at $656 \mathrm{~nm}$. A broadened $\mathrm{H}_{\beta}$ line was also visible at $484 \mathrm{~nm}$. In the infrared region, the four distinct lines could be observed due to atomic oxygen with the strongest emission at $777 \mathrm{~nm}$. No features were present in the UV range other than the $\mathrm{OH}$ line at $306 \mathrm{~nm}$ and the continuous decay of $\mathrm{H}_{\beta}$ line. Significant broadening of $\mathrm{H}_{\alpha}$ line was observed, which could be mainly attributed to Stark broadening, ${ }^{16}$ indicating a relatively high electron density inside the filament. The FWHM of the $\mathrm{H}_{\alpha}$ line was measured as $16 \mathrm{~nm}$, corresponding to an estimated electron density of about $10^{19} \mathrm{~cm}^{-3}$.

Inactivation experiments were carried out for different E. coli concentrations ranging from $10^{4}$ to $10^{8} \mathrm{CFU} / \mathrm{ml}$.

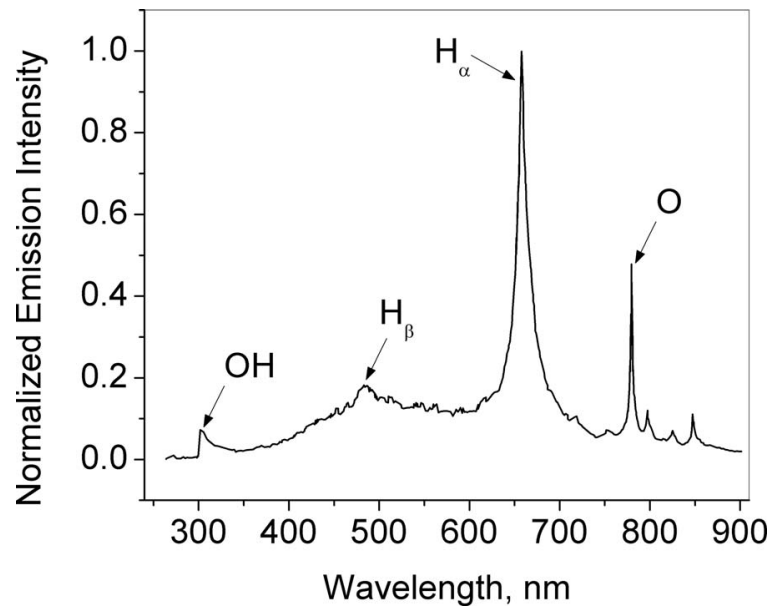

FIG. 4. Emission spectrum of the plasma array in distilled water. 


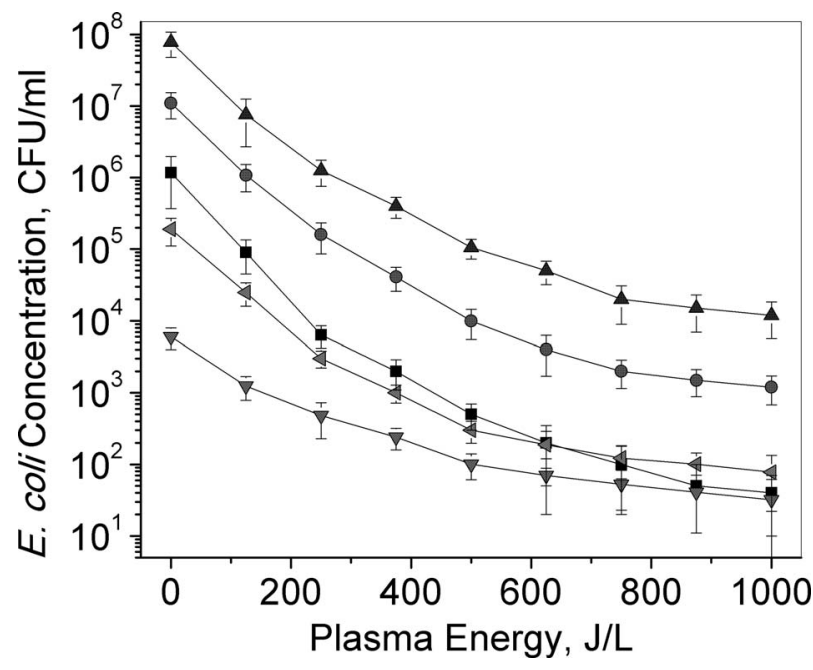

FIG. 5. Survival plot of $E$. coli for different initial bacterial concentrations.

For the sterilization experiments, a non-pathogenic strain of E. coli bacterium, K12, was used. Cell stocks of $E$. coli were prepared by incubating cultures in Luria-Bertani-Miller broth for $20-22 \mathrm{hr}$ at $37^{\circ} \mathrm{C}$. E. coli obtained in stationary phase were then centrifuged, washed in sterile distilled water, and finally re-suspended to population densities from $10^{4}$ to $10^{8}$ $\mathrm{CFU} / \mathrm{ml}$. In each test, $\mathrm{NaCl}$ was added to the $500-\mathrm{mL}$ water sample to adjust the conductivity to $200 \mu \mathrm{S} / \mathrm{cm}$. To analyze the treatment results, serial dilutions of the samples were prepared using sterile water and enumerated using a standard spread plate counting method. All dilution and plating were performed within 30 min after the plasma treatment. All experiments were repeated three times to ensure the reproducibility of the results. A consistent $p \mathrm{H}$ drop of $0.2-0.4$ was observed for all cases.

Figure 5 shows the survival curves obtained for different concentrations. The effectiveness of the spark discharge in inactivating E. coli is expressed as D-value, which is defined as the energy required to achieve one log reduction in bacterial concentration at the specific plasma treatment condition. The minimum D-value of $148 \mathrm{~J} / \mathrm{L}$ was achieved at a concentration of $10^{6} \mathrm{CFU} / \mathrm{ml}$. The value increased to $174 \mathrm{~J} / \mathrm{L}$ and $339 \mathrm{~J} / \mathrm{L}$ for concentrations of $10^{8}$ and $10^{4} \mathrm{CFU} / \mathrm{ml}$, respectively. The results clearly indicate the effectiveness of the discharge array in $E$. coli sterilization. The dependence of sterilization energy cost on bacterial concentrations can be explained by the competition between reactive species and UV radiations. ${ }^{17}$ The reactive species have direct impact on the outer membranes of microorganisms, while UV photons can cause irreparable damage to the bacterial DNA. At lower bacterial concentrations, the sterilization efficiency is limited by the short life- time of reactive species in water. At higher bacterial concentrations, the turbidity of the solution increases significantly. As a result, despite the fact that pure liquid water is largely transparent over 200-800 nm range with an absorption coefficient less than $0.01 \mathrm{~cm}^{-1}$, the sterilization efficiency may be decreased by the inability of UV radiation to reach $E$. coli due to the poor transparency. Additionally, at higher concentrations, E. coli can aggregate among themselves, partially shielding and thus preventing the active species from effectively destroying the microorganisms.

In conclusion, a low-cost technique to produce largevolume pulsed discharge array in water was demonstrated. The system utilizes thin metal disk electrodes to generate high electric field at the periphery of the disk, thus avoiding the deterioration of electric field strength usually associated with the erosion of conventional point electrodes. By stacking multiple metal disks, the system can be easily scaled up for large-scale water treatment applications. Sterilization experiments were performed and it was shown that up to 5-log reduction of bacterial counts could be achieved with D-values of 148-339 J/L over a wide range of $E$. coli concentrations from $10^{4}$ to $10^{8}$ $\mathrm{CFU} / \mathrm{ml}$.

This work was supported by U.S. Department of Energy, National Energy Technology Laboratory, through Contract No. DE-NT0005308.

${ }^{1}$ M. Laroussi, IEEE Trans. Plasma Sci. 24, 1188 (1996).

${ }^{2}$ M. A. Malik, A. Ghaffar, and S. A. Malik, Plasma Sources Sci. Technol. 10, 82 (2001).

${ }^{3}$ S. Masayuki, Plasma Sources Sci. Technol. 17, 024021 (2008).

${ }^{4}$ M. Moisan, J. Barbeau, M.-C. Crevier, J. Pelletier, N. Philip, and B. Saoudi, Pure Appl. Chem. 74, 349 (2002).

${ }^{5}$ P. Bruggeman and C. Leys, J. Phys. D: Appl. Phys. 42, 053001 (2009).

${ }^{6}$ Y. C. Hong, H. J. Park, B. J. Lee, W. S. Kang, and H. S. Uhm, Phys. Plasmas 17, 053502 (2010).

${ }^{7}$ B. R. Locke, M. Sato, P. Sunka, M. R. Hoffmann, and J. S. Chang, Ind. Eng. Chem. Res. 45, 882 (2006).

${ }^{8}$ O. Sakai, M. Kimura, T. Shirafuji, and K. Tachibana, Appl. Phys. Lett. 93, 231501 (2008).

${ }^{9}$ Y. Sakiyama, T. Tomai, M. Miyano, and D. B. Graves, Appl. Phys. Lett. 94, 161501 (2009).

${ }^{10}$ M. A. Malik, Y. Minamitani, S. Xiao, J. F. Kolb, and K. H. Schoenbach, IEEE Trans. Plasma Sci. 33, 490 (2005).

${ }^{11}$ P. Lukes, M. Clupek, V. Babicky, and P. Sunka, IEEE Trans. Plasma Sci. 36, 1146 (2008)

${ }^{12}$ V. Goryachev, A. Ufimtsev, and A. Khodakovskii, Tech. Phys. Lett. 23, 386 (1997).

${ }^{13}$ A. Starikovskiy, Y. Yang, Y. Cho, and A. Fridman, Plasma Sources Sci. Technol. 20, 024003 (2011).

${ }^{14}$ H. M. Jones and E. E. Kunhardt, J. Phys. D: Appl. Phys. 28, 178 (1995).

${ }^{15}$ T. Shirafuji, T. Kitagawa, T. Wakai, and K. Tachibana, Appl. Phys. Lett. 83, 2309 (2003).

${ }^{16}$ P. Barmann, S. Kroll, and A. Sunesson, J. Phys. D: Appl. Phys. 29, 1188 (1996).

${ }^{17}$ M. Laroussi and F. Leipold, Int. J. Mass Spectrom. 233, 81 (2004). 


\title{
Nonequilibrium Liquid Plasma Generation
}

\author{
Andrey Starikovskiy, Yong Yang, Young I. Cho, and Alexander Fridman
}

\begin{abstract}
In most cases, the electric breakdown of liquids is initiated by the application of high electric field on the electrode, followed by rapid propagation and branching of plasma channels. Typically, plasmas are only considered to exist through the ionization of gases, and typical production of plasmas in liquids has generated bubbles through heating or via cavitations and sustains the plasmas within those bubbles. The question appears: Is it possible to ionize the liquid without cracking and voids formation? We have demonstrated possibility of formation of nonequilibrium plasma in liquid phase and investigated the dynamics of excitation and quenching of nonequilibrium plasma in liquid water.
\end{abstract}

Index Terms-Liquid plasma, nonequilibrium plasma, pulsed picosecond discharge.

D EVELOPMENTS of discharges in liquids were performed for a long time [1]. The difference of the current approach is using picosecond high-voltage pulsed-power systems [2], which guarantee absence of voids. First, the power supply generates pulses with $+16-\mathrm{kV}$ pulse amplitude in $50-\Omega$ coaxial cable (up to $32 \mathrm{kV}$ on the high-voltage electrode tip because of pulse reflection), 10-ns pulse duration (90\% amplitude), 0.3-ns rise time, and 3-ns fall time. The second system generates $+112-\mathrm{kV}$ pulses (224 $\mathrm{kV}$ on the gap) with 150-ps rise time and duration on the half-height of about 400 ps. In all experiments, we used the pulse frequency $f=1 \mathrm{~Hz}$.

The discharge cell had a point-to-plate geometry with a high-voltage electrode diameter of $100 \mu \mathrm{m}$. The high-voltage electrode was a cutoff platinum rod. The interelectrode distance was $4 \mathrm{~mm}$; the low-voltage electrode diameter was $18 \mathrm{~mm}$ (Fig. 1). The discharge cell has a couple of quartz windows (the thickness was $1 \mathrm{~mm}$ ). The water layer between the center of the discharge gap and the window was $50 \mathrm{~mm}$. We used doubledistilled water for these experiments without deionization or $\mathrm{pH}$ adjustment $(p H \sim 6.4$ and conductivity $\sim 5 \mu \mathrm{S})$.

The measurements were performed with the help of a 4 Picos ICCD camera by Stanford Computer Optics. The camera has an 18-mm-diameter multialkaline photocathode with a spectral response from 180 to $750 \mathrm{~nm}$. A quartz lens with a focal distance of $70 \mathrm{~mm}$ and a diameter of $50 \mathrm{~mm}$ was used to focus the discharge gap to the photocathode with four-times

Manuscript received December 1, 2010; revised May 13, 2011; accepted May 24, 2011. Date of publication August 12, 2011; date of current version November 9, 2011. This work was supported in part by the National Science Foundation, by the Air Force Office of Scientific Research, and by the U.S. Department of Energy.

A. Starikovskiy is with the Department of Mechanical and Aerospace Engineering, Princeton University, Princeton, NJ 08544 USA (e-mail: astariko@ princeton.edu).

Y. Yang, Y. I. Cho, and A. Fridman are with the Department of Mechanical Engineering and Mechanics, Drexel University, Philadelphia, PA 19104 USA (e-mail: yy65@drexel.edu; choyi@drexel.edu; af55@drexel.edu).

Color versions of one or more of the figures in this paper are available online at http://ieeexplore.iee.org.

Digital Object Identifier 10.1109/TPS.2011.2160741 magnification. Typical camera's field of view was $2.6 \times 1.7 \mathrm{~mm}$ (Figs. 2 and 3); the spectral response was $250-750 \mathrm{~nm}$, taking into account UV-emission absorption in the water layer.

It was found that discharge in distilled degassed liquid water develops in picosecond time scale. The size of the excited region near the tip of the high-voltage electrode was $\sim 1 \mathrm{~mm}$. The discharge has a complex multichannel structure, and this structure has slightly changed from pulse to pulse; however, the discharge had almost the same dynamics (Fig. 2). To analyze the spatial structure of the discharge, we used a longer camera gate $(1 \mathrm{~ns})$ without signal accumulation. Typical emitting channel's diameter was $\sim 50 \mu \mathrm{m}$, and the propagation length was $0.5-0.6 \mathrm{~mm}$ for $U=27 \mathrm{kV}$. In the case of short rise time, we observed discharge propagation with a velocity up to $2000 \mathrm{~km} / \mathrm{s}$ $(2 \mathrm{~mm} / \mathrm{ns})$ during the very initial stage of the discharge corresponding to the voltage rise time (Fig. 2). The propagation velocity was estimated as an integral speed of the plasma channel head. When voltage reaches the maximum, the discharge propagation stops, and the "dark phase" appears. During this phase, the discharge cannot propagate because of space charge formation and electric field decrease. The voltage decrease leads to the return stroke formation and second emission phase. This means that the channels lost the conductivity and the trailing edge of the nanosecond pulse generates significant electric field and excitation of the media which is comparable to the excitation corresponding to the leading edge of the pulse. The experiments at elevated voltage prove the discharge propagation in the liquid phase. As in the case with 10-ns pulses, no bubble formation was observed. Fig. 3 shows the dynamics of the discharge formation for $U=224 \mathrm{kV}$. The $\Lambda$-shaped pulse has no plateau, and the dark phase of the discharge did not appear (Fig. 3). Discharge development takes an extremely short time (in 100-200 ps, the plasma channels reach the length of $0.5-0.8 \mathrm{~mm}$ and the diameter of $\sim 100 \mu \mathrm{m}$ ).

\section{REFERENCES}

[1] P. Bruggeman and C. Leys, "Non-thermal plasmas in and in contact with liquids," J. Phys. D, Appl. Phys., vol. 42, no. 5, p. 053001, Mar. 2009.

[2] A. Starikovskiy, Y. Yang, Y. Cho, and A. Fridman, "Nonequilibrium plasma in liquid water-Dynamics of generation and quenching," Plasma Sources Sci. Technol., vol. 20, no. 2, p. 024003, Apr. 2011. 

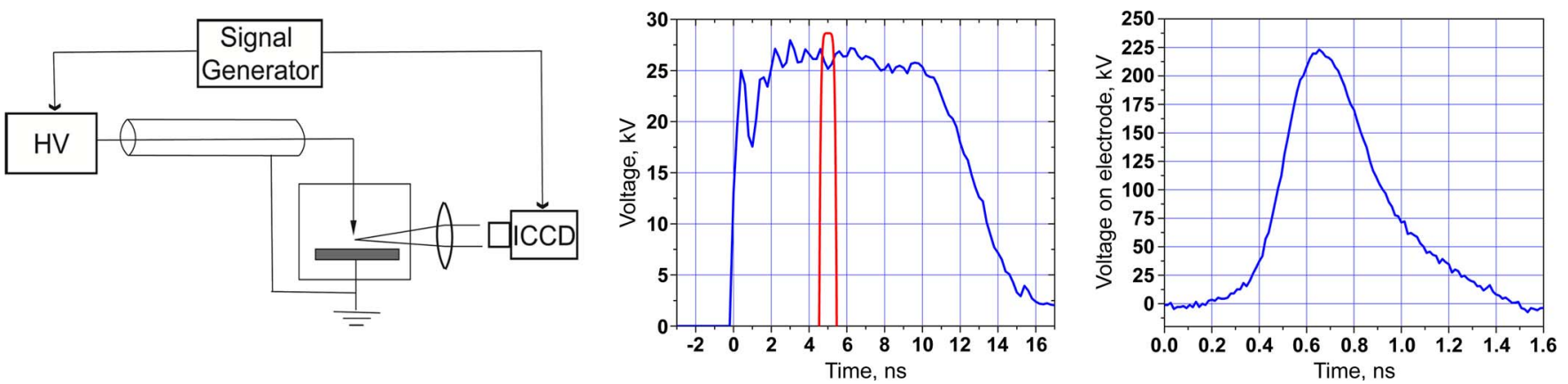

Fig. 1. (a) Schematic of the experimental setup. (b) Ten-nanosecond pulse shape. (Red curve) ICCD gate shape. (c) 0.4-ns pulse shape.

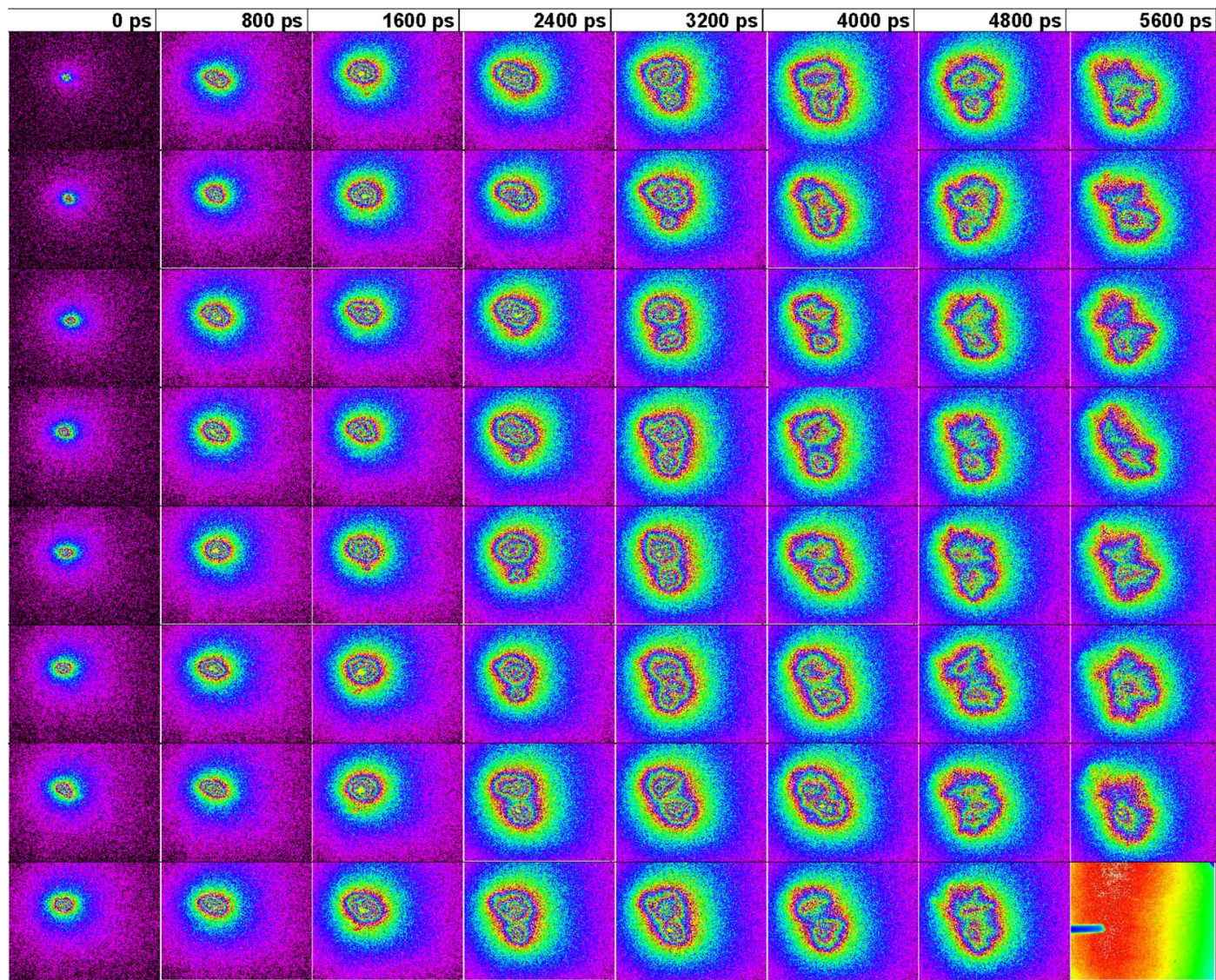

Fig. 2. Dynamics of discharge emission. Distilled water. $U=27 \mathrm{kV}$. The camera gate is $1 \mathrm{~ns}$. The spectral response is $250-750 \mathrm{~nm}$. Each row is shifted with respect to previous by $100 \mathrm{ps}$. The lower right image shows a picture of a 100- $\mu$ m electrode.

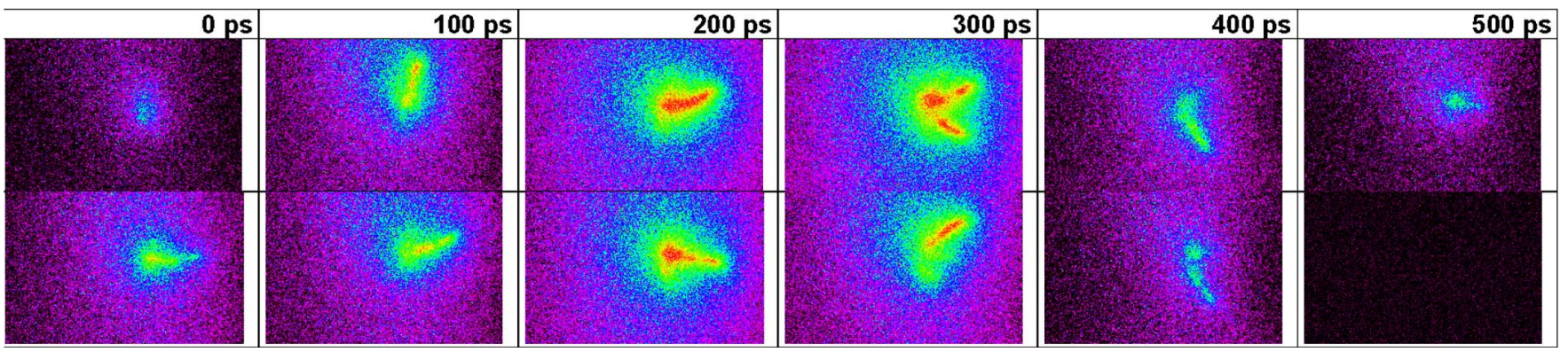

Fig. 3. Dynamics of discharge emission. Distilled water. $U=220 \mathrm{kV}$. $d U / d t=1.46 \mathrm{MV} / \mathrm{ns}$. The camera gate is $500 \mathrm{ps}$. The time shift between frames is 50 ps. The second row is shifted with respect to the first by $50 \mathrm{ps}$. 


\title{
Plasma Discharge in Water
}

\author{
YONG YANG, ALEXANDER FRIDMAN, and YOUNG I. CHO* \\ Department of Mechanical Engineering and Mechanics, Drexel University, \\ Philadelphia, PA 19104, USA
}

\section{Introduction}

\section{A. Needs for Plasma Water Treatment}

\section{Cooling Water Management}

Water is used as a cooling medium in large centralized air-conditioning systems as well as in thermoelectric power plants. In both cases, the cooling water plays an essential role in removing heat from condensers. Since the evaporation of pure water is the basic means to remove heat from the condensers, the concentration of mineral ions in circulating cooling water increases with time, resulting in hard water within a week even if soft water is used as makeup water. Hence, a part of the circulating water is periodically or continuously discharged in order to maintain the proper concentration of the mineral ions in circulating cooling water in the form of blowdown.

Thermoelectric power plants produce about half of the nation's electricity. According to the US Geological Survey's (USGS) water use survey data [1], thermoelectric generation accounted for 39\% (136 billion gallons per day [BGD]) of all freshwater withdrawals in the nation in 2000, second only to irrigation (see Fig. 1) [1]. Furthermore, the average daily national freshwater consumption for thermoelectric power generation is predicted to increase from the current $4 \mathrm{BGD}$ for the production of approximately $720 \mathrm{GW}$ electricity to 8 BGD for $840 \mathrm{GW}$ in 2030 (see Fig. 2) [2].

In the cooling water management, it is important to distinguish between water withdrawal and water consumption. Water withdrawal represents the total water taken from a source, while water consumption represents the amount of water withdrawal that is not returned to the source. Freshwater consumption for the year 1995 (the most recent year for which these data are available) is presented in Fig. 3. Freshwater consumption for thermoelectric

*Current address: Department of Mechanical Engineering and Mechanics, Drexel University, Philadelphia, PA 19104, USA 


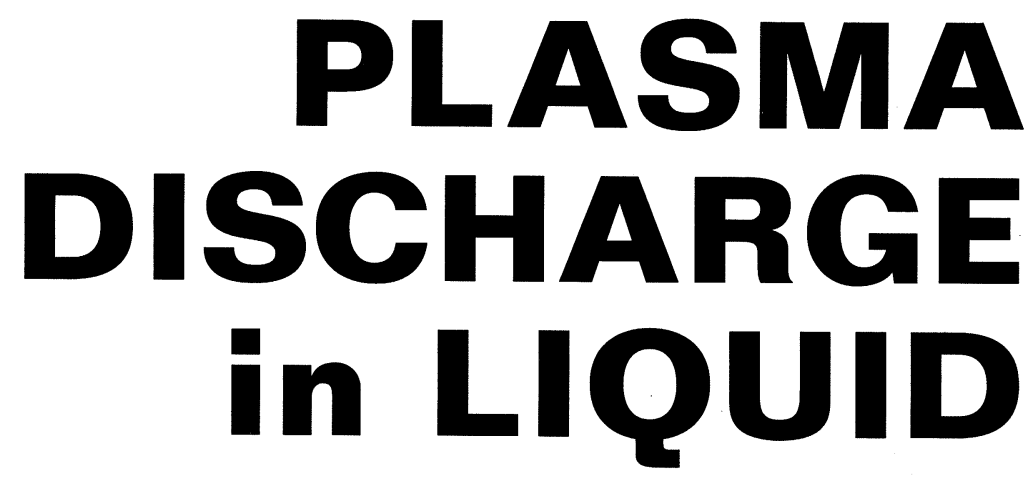

\section{Water Treatment and Applications}

\section{Yong Yang Young I. Cho Alexander Fridman}

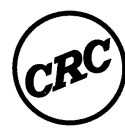

CRC Press

Taylor \& Francis Group

Boca Raton London New York

CRC Press is an imprint of the

Taylor \& Francis Group, an informa business 